
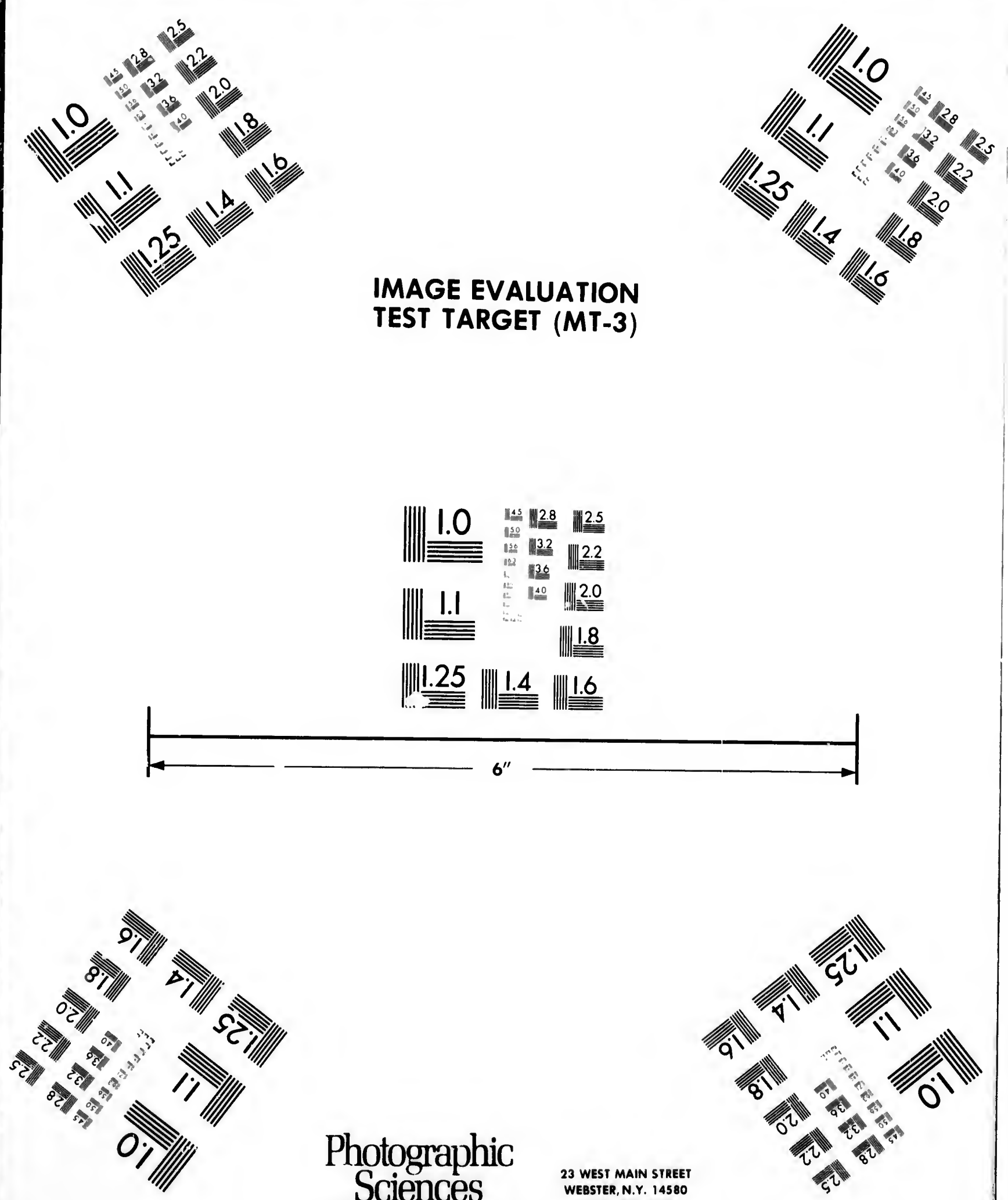

Photographic Sciences Corporation
23 WEST MAIN STREET WEBSTER, N.Y. 14580 (716) 872-4503

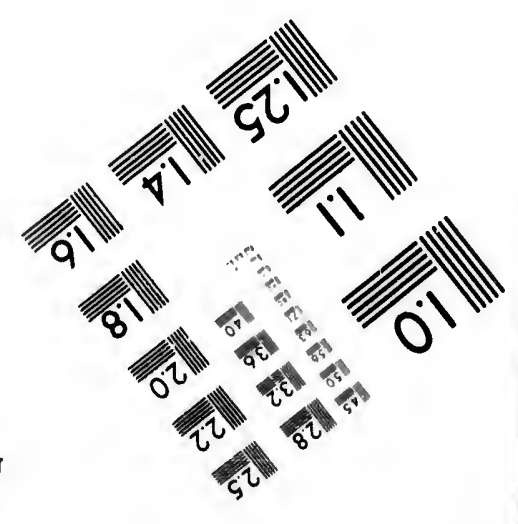



Series.

\section{CIHM/ICMH Collection de microfiches.}

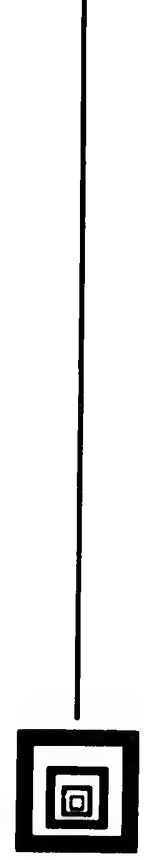

Canadian Institute for His:orical Microreproductions / Institut canadien de microreproductions historiques
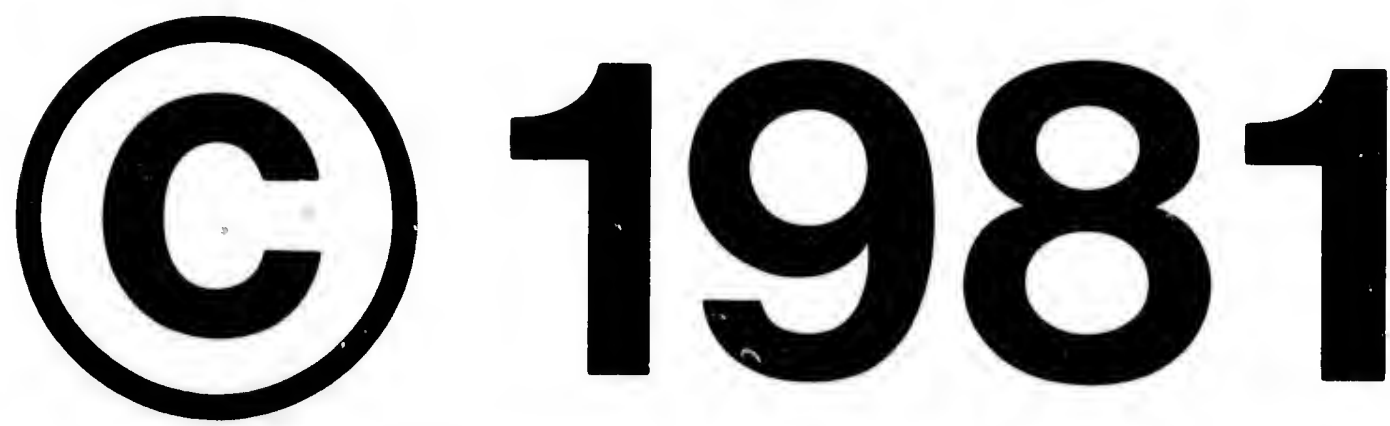
The Institute has attempted to obtain the best original copy available for filming. Features of this copy which may be bibliographically unique, which may alter any of the images in the reproduction, or which may significantly change the usual method of filming, are checked below.

\section{Coloured covers/}

Couverture de couleur

Covers damaged/

Couverture endommagée

Covers restored and/or laminated/

Couverture restaurée et/ou pelliculée

Cover title missing/

Le titre de couverture manque

Coloured maps/

Cartes géographiques en couleur

Coloured ink (i.e. other than blue or black)/

Encre de couleur (i.e. autre que bleue ou noire)

Coloured plates and/or illustrations/

Planches et/ou illustrations en couleur

Bound with other material/

Relié avec d'autres documents

Tight binding may cause shadows or distortion along interior margin/

La reliure serrée peut causer de l'ombre ou de la distortion le long de la marge intérieure

Blank leaves added during restoration may appear within the text. Whenever possible, these have been omitted from filming/

II se peut que certaines pages blanches ajoutées lors d'une restauration apparaissent dans le texte, mais, lorsque cela était possible, ces pages n'ont pas été filmées.

Additional comments:/

Commentaires supplémentaires:
L'Institut a microfilmé le meilleur exemplaire qu'il lui a été possible de se procurer. Les détails de cet exemplaire qui sont peut-être uniques du point de vue bibliographique, qui peuvent modifier une image reproduite, ou qui peuvent exiger une modification dans la méthode normale de filmage sont indiqués ci-dessous.

Coloured pages/

Pages de couleur

Pages damaged/

Pages endommagées

Pages restored and/or laminated/

Pages restaurées et/ou pelliculées

Pages discoloured, stained or foxed/

Pages décolorées, tachetées ou piquées

\section{Pages detached/}

Pages détachées

\section{Showthrough/}

Transparencs

Quality of print varies/

Qualité inégale de l'impression

Includes supplementary material/

Comprend du matériel supplémentaire

Only edition available'

Seule édition disponible

Pages wholly or partially obscured by errata slips, tissues, etc.. have been refilmed to ensure the best possible image/

Les pages totalement ou partiellement obscurcies par un feuillet d'errata, une pelure, etc., ont été filmées à nouveau de façon à obtenir la meilleure image possible.

Irregular Paging :

[1] - 110, 139-147

This item is filmed at the reduction ratio checked below/ Ce document est filmé au taux de réduction indiqué ci-dessous.

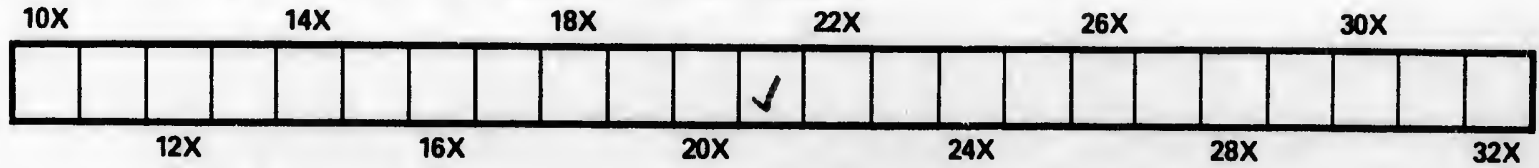


The copy filmed here has been reproduced thanks to the generosity of:

\section{Entomology Research Library Agriculture Canada}

The images appearing here are the best quality possible considering the condition and legibility of the original copy and in keeping with the filming contract specifications.

Original copies in printed paper covers are filmed beginning with the front cover and ending on the last page with a printed or illustrated impres. sion, or the back cover when appropriate. All other original copies are filmed beginning on the first page with a printed or illustrated impression, and ending on the last page with a printed or illustrated impression.

The last recorded frame on each microfiche shall contain the symbol $\rightarrow$ Imeaning "CON. TINUED"), or the symbol $\nabla$ (meaning "END"), whichever applies.

Maps, plates, charts, etc., may be filmed at different reduction ratios. Those too large to be entirely included in one exposure are filmed beginning in the upper left hand corner, left to right and top to bottom, as many frames as required. The following diagrams illustrate the method:
L'exemplaire filmé fut reproduit grâce á le générosité de:

Bibliothèque de recherche entomologique Agriculture Canada

Les images suivantes ont été reproduites avec le plus grand soin, compte tenu de la condition et de la netteté de l'exemplaire filmé, et en conformité avec les conditions du contrat de filmage.

Les exemplaires originaux dont la couverture en papier est imprimé sont filmés en commençant par le premier plat et en terminant soit par la derniére page qui comporte une empreinte d'impression ou d'illustration, soit par le second plat, selon le cas. Tous les autres exemplaires originaux sont filmés en commençant par la première page qui comporte une ampreinte d'impression ou d'illustration et en terminant par la dernière page qui comporte une telle empreinte.

Un des symboles suivants apparaîtra sur la derniere image de chaque microfiche, selon le cas: le symbole $\rightarrow$ signifie "A SUIVRE", le symbole $\nabla$ signifie "FIN".

Les cartes, planches, tableaux, etc., peuvent être filmés à des taux de réduction différents. Lorsque le document ast trop grand pour être reproduit en un seul cliché, il est filmé à partir de l'angle supérieur gauche, de gauche a droite, et de haut en bas, on prenant le nombre d'images nécessaire. Les diagrammes suivants illustrent la méthode.

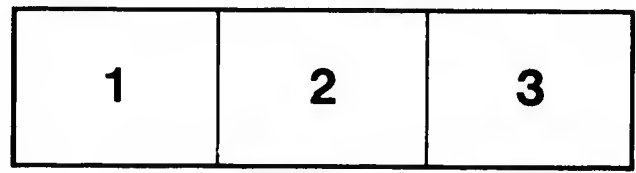

\begin{tabular}{|l|l|l|}
\hline 1 & 2 & 3 \\
\hline 4 & 5 & 6 \\
\hline
\end{tabular}

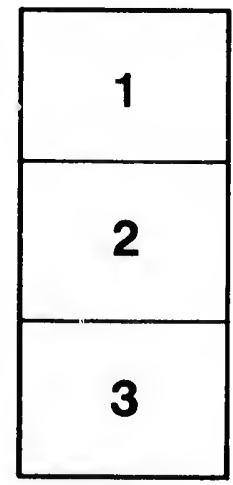


UN

BíBLIO 
SMITHSONIAN INSTITUTION.

UNITED STATES NATIONAL MUSEUM.

\section{BULLETIN}

OF THF:

\section{UNITED S'TATES NATIONAL. MUSEUM.}

No. 35.

BIBLIOGRAPHICAL CATALOGUE OF THE DESCRIBEI TRANSFORILTIONS

OF NORTH AUERICAN LEPIDOPTERA.

$\mathbf{1 3 Y}$

HENRY FDWARDS.

WASHINGTON :

GOVERNMFNT PRINTING OFFICE. 1889.

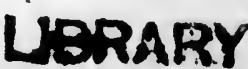




\section{ADTERTISEMENT.}

The present publication (Bulletin No. 35) is the forty-sixth of a series of papers intended to illustrate the collections belonging to the United States, and constituting the National Museum, of which the Smithsonian Institution was placed in charge by the act of Congress of August 10, 1846 .

The publications of the National Museum consist of two seriesthe Bulletins, of which this is No. 35, in continuons series, and the Proceedings, of which the eleventh volume is now in press.

The volumes of Proceedings are printed, signature by signature, each issue haring its orn clate, and a small edition of each signature is distributed to libraries promptly after its publication.

Full lists of the publications of the Mnseum may be found in the current catalogues of the publications of the Smithsonian Institution.

Papers intended for publication in the Proceedings and Bulletins of the National Musenm are referred to the Committee on Publications, consisting of the following members: T. H. Bean, A. Howard Clark (editor), Otis T. Mason, John Mrurdoch, Leonhard Stejneger, Frederick W. True, and Lester F. Ward.

$$
\begin{gathered}
\text { S. P. LANGLEY, } \\
\text { Secretary of the Smithsomian Institution. }
\end{gathered}
$$

Washington, Jamuary 23, 1989. 


\title{
BIBLIOGRAPHICAL CATALOGUE
}

\author{
OF THE \\ DESCRIBED TRANSFORMATIONS
}

OF

\section{NORTH ANERICAN LEPIDOPTERA.}

BY

HENRY EDTWARUS.

WASHINGTON :

GOVERNMENT PRINTING OFFICE. I 889 . 
O 81 . $6 E 26$ 


\section{PREFACE.}

I believe that the present Catalogue will supp.y a want that has long been felt by many entomologists, and though it may be far from perfect, it will, I trust, be acceptable to the students of the earlier stages of North American Lepidoptera. In its compilation I have occupied a good portion of the spare time at my command for three years past, and have carefully examined every publication that has been accessible to me. I know that many references may possibly have been omitted, the works in which they occur not being within my reaeh, but I trust that few if any species have been overlooked altogether. Should such be the case, however, I shall be greatly indebted to my entomological friends if they will point them out to me, as it is my intention to issue a supplement to this list once a year, and thus keep up with the knowledge of what deseriptions have been recorded. In the nomenclature I have followed the catalogue of W. H. Edwards in the Diurne; Grote's Check List ( 1882 ) in the Macro-Lepiduptera; Professor.Fernald in the Tortricide; Stainton, Clemens, and Chambers in the Tineide; and Lord Walsingham in the Pterophoride. Of the two latter families no catalogue of our species exists, and I therefore ask for indulgence should any errors in the sequence of the genera or otherwise be detected. It has been my aim to make the references as clear but at the same time as brief as possible, and I have, as will be seen, arranged all descriptions according to the date of their publication. When the words (quistes Harris, e.g.) oceur after the name of the describer, it will be understood that the text of the description has been used; but when the words (after Riley or Abb. Sm., etc.) are found, it will mean that the drating or chy rating has been borrowed from these authors. In all the species, where known to me, the food-plant has been giren, except in the case of the Arctias and in Agrotis, the former being almost general feeders, and the latter living chiefly on grasses and low herbaceous plants. If a reference is followed by the word (brief), it signifies that the same is but a mere mention, of ten perhaps only a food-plant, but it has been cleemed advisable to include such notices. In the appendix are a few species not referable to any known insects, and I have added a short list of some papers which may be of value to the students of transformations. I should like also to call attention to the admirable "life histories" by Messrs. Riley, French, Edwards, Saunders, Packard, H. H. Clark, and others, which are scattered through Entomological Reports, both State and National, the Canadian Entomologist, "Papilio," "Entomologica Americana," and kindred publications, papers which may advantageously be studied by future 
describers as models of style and accuracy. I desire to tender my warmest thanks to Professor Riley, Mr. S. H. Seudder, Mr. J. B. Smith, Mr. W. H. Edwards, and Mr. W. Beutenmüller for much valuable aid, both in the loan of publications and the copying of references, thus greatly assisting me in my task.

I earnestly hope that the publication of this catalogue will be the means of inducing observers to take a wider and more extended interest in the preparatory stages, and to place as early as possible upon record the results of their studies. It will be readily seen that though, up to the end of the Brirlycida, a good deal has already been done, the knowledge of the transformations of the Noctuide and succeeding families is slight indeed, and it is to these groups that attention is especially directed. Should this list be instrumental in adding to our information regarding the many species of Lepidoptera, of whose earlier stages we now know so little, my labor will be rewarded, and the many hours passec in its preparation will not have been sccupied in vain.

HENRY EDWARDS 


\section{NCMBER OF SPECIES OF WHICH FARLIER STAGES ARE RLCOIDED IN THIS CATALOGCE.}

NAм. No. of apecles.

Rhopalocera.......................................180

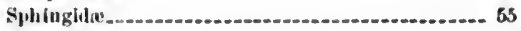

Figeriade .......................................... 16

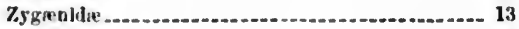

Bomliyces _............................................ 178

Noctuida

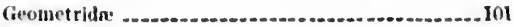

Pyralfila ........................................... 33

Tortricilla

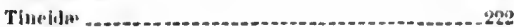

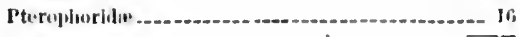

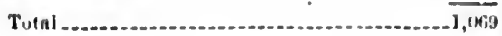

PRINCIPAL ACTHORS IXII I'CHLICATIONS QCOTED.

Acanemy Nat. Sciescee, Pulta., Proc.

Antot AND SMitil. Lefldepterous linects of Georgla. Amenicas Extos. Soc., Trampations of.

Amficas Extomologist.

American Assoc. Anv. Scr., Proceedingz of.

Amentcan Naturatist.

Baldari, Mns. J. l'. Insect Lives.

Berutse, C. J. S. Papers In Cannd. Fntom.

Reports Entom. Soc, Ontario.

Bolentvat, Dr. J. A. Itjidog̨tora de la Californie. Speciles General, Ithojulocera.

Specles General, lleterucera.

Bossd'val, Dr., AND J. Leconts. IIst. Lepid. N. America.

Boston Snc. Nat. ILstoky., Proc,

BRookryn Extom. Soc., Bulletln of.

Betfalo Soc. Nat, Hist. Bulletín of.

Caltfornia acal, Science, Proc. of, vols. 1-6.

Canapian Evtomologist, vols, 1-2].

Cnapmax, A. W. Jajers In Canadian Eutomologist. Cuent-Lteas-Demanets. Encye. Hist. Nat., vols, ]-2.

Clemens Brackexrinos. Tinelde of $\mathbf{N}$. Amer., (edit. Staluton.)

Papers in l'roc. Am. Futom. Soc.

Cossrock, J. II. Rejt. Entomologist, C. S. Dept. Agr., 1879-80.

Report on Cotton Insects.

Insects Injurieun to Sugar Cane.

Cook, A. J. Papers in Mllsigan Reports.

Coqtut.Letr, D.W. Pupers In Trans. Dept. Agr., Illinois.

Papiers in Papllio.

Cratis, Joun. British Entomology.

Cutniso, Hiras, M. D. Pests of Farm. X. Hamp. Bul. Agr.

Davenport Acav. Xat. Sciences, Proceeliugs of, rols. 1-5.

Descas, Juя Ne. Naturalists' Library. (Elitor.)

Drunx, Drv. Illustr, Exotic Entomology, (West wool edit.)

Edwards, II enry, Papers in Proc. Cal. Acad. Sclences, Papers in Papillo.

Papers in Entomologica Americana.

Kingsley's Standard Natural History, vol. 2. linwand, Wy. It. Pazers in Proc, Eutom. Soc. Phila. Hupers in Trunx. An. Bntom. Sor.

P'ajers in C'numbian Finton.

P'ajers in P'upilio.

Buttertices of $\mathbf{X}$. America, merles 1, 2, 3 .

Elwes, II. J. I'ajere in l'roc. Zo'ilon. Soc. London. Emsoxe, E. Naturul llist. N. York, Entom.

Estogiolnica Americana, vols. I-4.

Extomological sic. P'ulla., l'ros. of, rols. 1-6.

F.мтом. Joxisted, (Wen, vols. 1-x.

Fersaldi, 6. H. Payers in Canadlan Entom. Papers in I'apilio.

Kinguley's Stmulurd Natural IIistory, rol, 2. Buttertiles of Maine.

Spluingide of $\mathbf{N}$, Englanu.

Field axn Forest, (alitor, C. R. Dollge.)

Figvier, I. The Insect Wi rld. (Traun].)

Fitci, Aks. Reports on Inzects of N. Yoik, role. 1-14. lagers in Trans, $\mathbf{x}, \mathbf{Y}$. Stute Agr. Soc.

Fietcing, J. It(y)ts. Fintom. Ontario.

Trijs to Nepigon, I $\times 88$.

Frexci, G. H. Butterties of Eantern U. States. Pupers in Canad. Entom. Prepers in Papillo.

Frues, T. W. J'apers In C'anal. Eutom.

Gsilorb, W. lteport in Trahs, N. Y.State Agr. Soc.

Glovea, Towsesp. Jaeports of Entomologlst C. S. Il, jt. Agr.

Goomerr., L. W. Papers in Papillo and Canad. Enton.

Gossr., p. 11. Connadian Nituralist. Latters from Alubama.

Grots, Ace. R. Pajers in Proc, Entom. Soc. Phila. Japers in Cunnd. Entomol.

Papers in N. American Entomologlist. Papers in P'apilio.

Gesntaci, Jean. Entomologla Cubana.

Harria, Tuanneus W. Insects Injurions to Vegetation. Pagers In Trnns. N. Y. State Agr. Soc., etr. Entomologica] Correspondence.

IIURRarI, II. G. Insects affectlng the Orange. II TMPurevs, Nozl. Genera of Britlel, Moths. ILuxo18, Trans. Dept. Agrleulture of, Trans. State Agr. Soc, of. 


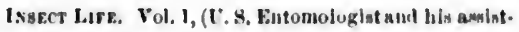
allts).

Iow A, Trnne. Horticu't. " c, of.

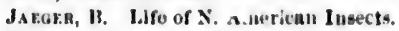

Kaxman. Trans. Kanmas Acud. Scleucty. Agricultural läjorts of.

Kraticky. Inureau of Agriculture.

Kıнау, W. F, Furop. Butt. an! Mołh.

Kol.an, V. Injurlous Innects. (Lumlun edislon.)

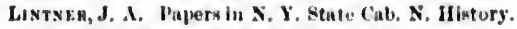
I'upers in Traus. S. Y. State Agr. Suc.

I'une re in Proc. Entom, Sx: Phila.

l'rpers in Tranm. Amer. Eintom. Soc.

Papers in Cunalian Entonodogist.

Pupers in Papllio.

Iiejits. N. Y. State Entom.

Maise. Reptr. Honril Agriculture.

Mans, B. Рickmas. I'njara in Payelie.

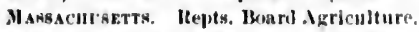

Mral, Tukonone L. J)uru. Lephl. Wheter's Geol. Surveg.

Mrougax. Reptis. Stute Bonrd Agrleniluyre.

Munlves, Mise Netrie. liepts. Dept. Agr., Illigols.

Misx Enot.. Statistles of.

Misit, f. J. Jajers in Amer. Noturulint.

II Insocen. Iivporta on Noxlous Rnd Ibeneficlal Insects, (Riley.)

Agrirultural Reports.

Monแu, IlEv. J. G. Symopsis X. Americun Lepid.

New Jenser, Agriculturul llounrts of.

New Yokk. Trank. Stut' Igr. Sec.

Stute Cabinet X. Ilistory.

Newsas, Eowaru. Ibitish Jlotla.

Nortu Canousa. Agricultural liegrotts.

Repurts Agric. Exjerim. sintion.

Ostano, Reports Fintom. Sine, of.

Otrawa. Trume. Field Nats. I'huh, vole. I-3.

Otтawa Natehalist, vole, i-it.

Lackand, Alpuztes S. Guide to Study of loserts.

Injurious Insets, new and little known.

Notes on Zygitulda.

Injurious Insects of the West, (IIayden's Iteportm.)

Ituif-hours with Insects.

Monograph of the Geometrid Mothe.

Iusects Injurlous to Forest anil Shude-trees.

Papizio, (edit. IIY. Filwarls and E. M. Aaron,) rols. 1-4.

Veale, Titias. Lepllogtera Amerlcuga.

P'rinativisia, Agr. Hipuren of.

Pors, Frinpe. Cent. Iaphld, cubaur.

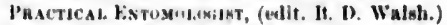

l'srcux, (edit. 13. I'. Mlanu, wis. 1-3.

IRatuvos, S. S. Repit. Agr. Penna.

Ratzeni un, J. Dho Forst-inanctell.

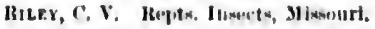

Papers In Tizhs. III. Fintum, Soc.

Papers in Casud. Entonologlst.

Propers lin l'mullio.

Sacsorns, Wy. Imsert* Injurious to Frults,

Pujwe in Canal. Katum.

Say, Tuemas. N. Amerleall Enton.

Scisat'x, Ws., Jn. Jajn'l's du l'apillo, ete.

Scconen, Sariz. II. P'apura in l'rov, Buaton N. HIst. Nor.

Prup.rs in Canal. Entour.

l'uperx in l'atjilin.

Butterflles, their nt ructuri, ite.

Silk Mastractine, Tuatiac on, Larilier'y C'sclojeelin.

Sмitu, Jils B. Monograph of Sphlughliי.

Staistos, II. T. British Jinterflies and Moths,

strenesk, J. F. Hlisstratlotas Brit. Fintoln.

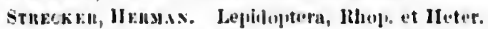

stuzte1, 16. II. Zyguniba anl Bombyclde S. Auer.

Tevsw, Saxnons. Maumal of Zobilugy.

Texs rus, (N.J.) Traus. S. IIIst. Six. of.

Tinsas, Crnis. P’uеers in Jllinols keports.

Venmost. Ilojte. Sitate IH]. Agr.

Viagisia, Aqrie, Replito of.

Walsisinas, Thos.an, Lonb. I'terophoride of ('u). Hud Oreng.

X. Auser, Tortricide Heteroc, Is, IIn.

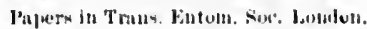

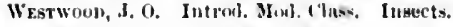

(Eilit. ly.) Drury's Exot. Entom.

Introrl. Fintum.

Wiscossis. Trans. Agr. Sor.

Woos, lix. J. G. Nitural Ili-t., vol, 2, (Entom.)

Insects at IIome.

Insects Abroud. 
) rols.

IIist.

yelo.

ner. 


\section{ER RATA.}

Page 17, line 10 from top-real Astragalus, Crotuluria for Astrayalus crotalaria.

Page 20, line 19 from top-read Aryynnis for Argynis.

Page 2', line 13 from top and line 27 from bottom-read C'sstilleia for Castileja and Castelejia.

Page 45, line 7 from top-read Ulmus americun for Ulmus umericanus.

Page 58, line 21 from top-read Enothera for Anothera.

Page 61, line 6 from top-read Asclepias tuberosa for dsclppias tuberosum.

Page 64, line 3 from top-read Quercus for Qucreus.

Page 67, top line-read Thyridopteryx for Thyridoptery.

Page 94, line 22 from top-read Crucifere for Cruciferca.

Page 94, line 27 from top-real Anarta for Ararta.

Page 109, line 1 from bottom-read Fuchsia for F'usclia.

Page 112, line 1 from bottom-read Oxycoccus for Occycoccus.

BULL, 35. 


\section{DESCRIBED TRANSFORMATIONS OF NORTH AMERICAN LEPIDOPTERA.}

\section{RHOPALOCERA.}

Papilio ajax. L. (and vars.)

1797. Larva, elirys., (col'd figs.) Abbot-Smith. Lep. Ins. Georgia, pl. 4.

1833. Larra, chrys., (col'd tigs.) Boirdural-Leconte. Ilist. Lep. N. Amer., pp. 4-8, pl. 1-2.

1836. Larva. Boislural. Spec. Gener. Dinrnie, rol. 1, pp. 25i-259.

1859. Larra, chrys., (figs.) I. H. Gosse. Letters from Alabama, p. 51.

1861. Larva. Jaeger. Life N. Amer. Ins., p. 209.

1971. Life hist., (col'd figs.) W. II. Edwarly. Butt. N. Amer., 1st series, pi. Papilio, 1, 2, 3.

1880. Larva. Miss N. Milllleton. Trans. Dep't Agr. Ill., vol. 18, Appendix, p. 74.

1884. Larva, (figs.) Dr. A. Gruber. Jena. Zeitschr. Nat. Copied in Papilio, vol. 4 , p. 88 .

1886. Life hist., (condensed.) G. H. French. Butt. East. U. States, p. 85.

Food Plavr. Asimina triloba. (Pawpaw.)

Papilio philenor. Fal.

1797. Larva. Abbot-Smith. Lep. Ins. (ieorgia, pl. 3.

1833. Larvi, (col'd figs.) Boi-duenl-Lecoute. Hist. Lep. N. Amer., p. 29, pl. 11.

1836. Larva, chrys. Boisdural. Spee. Gener. Diurnar, vol. 1, 1. 325.

1837. Larva, ehrys. Westrood, (quotes Abb.-Sm.) Drury's Ill. Exot. Entom., vol. 1 , p. 21.

1861. Larva. Morris, (quotes Bois.-Lec.) Synop. Lep. N. Amer., p. 6.

186t. Larva. Jeleger. Lite N. Amer. Ins., p. 209.

1869. Larva, ehrysalis, (figs.) Harris. Entom. Corres., 1. 273.

1870. Life history, (figs.) Riley. 2ll Missouri Rept., p. 116.

1873. Larva, chrysalis. Hy. Edurarls. Proc. Cal. Acad. Se,

1874. Chrysalis, (fig.) Scudder. Geology N. Hampshire, pl. A.

1875. Chrysalis. Iy. Edwards. Proc. Cal. Acad. Sc., vol. 5, p. 1.j2.

187T. Larva. G. H. French. Trans. Dept. Agr. Ill., vol. 15, p. 136.

1880. Larva, (figs.) D. W. Coquillett, (ı̨uotes Riley.) Trans. Dept. Agrr. Ill., rol. 18, Appendix, p. 164 .

1880. Larva, (figs.) Miss N. Mildllton. Trans. Dept. Agr. Ill., vol. 18, Appendix, p. 73 .

1880. Larva, ehrysalis, (figs.) T. Saunders. Rept. Entum. Soc. Ontario, 1. 37.

1881. Life hist. W. H. Edwerds. Canad. Entom., vol. 13, p. 9.

1881. Larva, chrysalis, (figs.) S. H. Scudder. Batterflies, their structure, etc., p. 22.

1881. Larva,(figs.) Dr. A. Gruber. Jena Zeitschr. Nat. Copiel in Papilio, vol. $4, \mathrm{p} .88$.

1886. Life history, (figs.) G. H. French. Butt. East. U. States, p. 88.

Foon Plasts. Species of Aristolochi i.

Papilio zolicaon. Bdv.

1873. Larva, chrysalis. Hy. Edwarls. Proc. Cal. Acad. Sc., vol. 5, p. 163.

1875. Larra, (col'd figs.) H. H. Edwarls. Bntt. N. Amer., 2d series, pl. Papilio, 6.

1884. Lifo hist. Ir. I. Edwards. Papilio, vol. 4, p. 162.

Food Praxts. Species of L'mbellifera. 
Papilio brevicauda. Saund.

1873. Egg, young larva, (figs.) H. Strecker, (quotes Couper in lit.) Rhopal.et Heteroc, pp. $49-69$, p]. 8.

1875. Egg, larva (col'd fig.) W. H. Elleaids. Butt. N. Amer., 2d scries, pl. Papilio, 8.

1880. Life hist., (col'd figs.) W. H. Edwarls. Butt. N. Amer., 2d series, part 9, pl. Papilio, 8 .

1883. Larva. P. H. Gose, in Jonrnal from 1832 to 1835 . Canad. Entom., vol. 15, p. 45.

1884. Larva, (l,rief.) Gruher. Jena Zeitschr. Nat. Copied in Papilio, vol.4, p. 86.

1885. Egg, larva. W. H. Edurards, (quotes Couper in lit.) Butt. N. Amer., 2d series, pl. 13.

Food Plan'ts. Various speeies of Lmbellifere.

Papilio machaon. L. var. aliaska. Seud.

1884. Larva, (col'il figs.) W. II. Edlcarls. Butt. N. Amer., 2d series, pl. 14.

Food Plaxt. Species of Umbelliferer.

Papilio oregonia. Edw.

1882. Larva, elirysalis. H. H. Streteh. Papilio, vol. 2, p. 120.

Fond Plant. "Wild parsnip."

Papilio asterias. Tab.

1833. Larva, ehrys., (col'd figs ) Boîsluval-Lecontc. Hist. Lep. N,Amer., p. 14, pl. 4.

1836. Larva, chrys. Boisdural. Spee. Gener. Diurnar, vol. 1, p. 333.

1841. Life history, (brief.) Harris. Ins. Inj. Vegretat., 1st edit., p. 212.

1852. Life history, (brief.) Harris. Ins. Inj. Vegetat., 2d edit., p. 231.

1857. Larva, ehrysalis, (figs.) Anonymous. Canad. Nat. and Geolog., vol. 2, p. 222.

1861. Larva. Morris, (quotes Bois.-Lec.) Synop. Lep. N. Amer., p. 6.

1832. Life listory, (col'd figs.) Harris. Ins. Inj. Vegetat., Flint's edit., p. $263, \mathrm{pl} .4$.

1864. Egg, larva, chrysalis. Lintuer. Proe. Entom. Soc. Phila., vol. 3, p. 51.

1864. Larva, (fig.) Jaeger. Life N. Amer. Ins., p. 208.

1867. Larva, chrys., (figs.) Sanborn Tenney. Mannal of Zoology, figs. 281, 282.

1869. Larva. Haris. Lintom. Corres., p. 270.

1873. Larva, chrysalis. Hy. Elwards. Proc. Cal. Aead. Se., vol. 5, p. 164.

18i6. Larva. Saunders. Rept. Entom. Soc. Ontario, p. 38.

1877. Larva. Packard. Inj. Ins. West, Hayden's Rept., p. 765.

1877. Larva. G. H. French. Trans. Dept. Agr. Ill., vol, 15, p. 137.

1878. Larra, elırys. Derritz. Zeitsch. gesament. natur.

1878. Larva, elırys., (figs.) H. Dertitz. Weigemann Archiv. Naturgesch, p. 4, pl. 1.

1879. Larva, elirysalis, (figs.) Mrs. J. P. Ballard. Insect Lives, p. 32.

1880. Larva. Miss N. Milllleton. Trans. Dept. Agr. Ill., App., vol. 18, 1. 74.

1880. Larra. D. W. Coquillett. Trans. Dept. Agr. Ill., rol. 18, App., p. 173.

1881. Larva, (figs.) S. H. Scudder. Butterflies, their strneture, ete., p. 2:.

1884. Egg, larva, chrys. C. H. Fernald. Butterflies of Maine, p. 22.

1884. Larra. Dr. A. Gruber. Jena Zeitsehr. Nat. Copied in Papilio, vol. 4, p. 84 .

1886. Life list., (figs.) H. French. Butt. East. U. States, p. 92.

Food Prasts. Varioụs species of Umbellifere.

Papilio troilus. L.

1797. Larva, ehrys, (eol'd figs) Abbot-Smith, (as P. ilioneus.) Lep. Ins. Georgia, pl. 2.

1833. Larva. Boisdural-Leconte. Hist. Lep. N. Amer., p. 26, pl. 10.

1836. Larva. Boisdural. Spec. Gener. Dinrnte, vol, 1, p. 335. 
1857. Larva, chrysalis, (figs.) Anonymous. Canad. Nat. und Geolog., vol. 2, p. 312.

1861. Larva. Morris, (quotes Bois.-Lec.) Synop. Lep. X. Amer., p. 5.

1862. Larva, chrysalis. Haris. Ins. Inj. Vegetat., Flint's edit., I. 26 i6.

1864. Larva. Jae'fer. Life X. A ner. Ins., p. 209.

1869. Larva. Suuniers, Canad. Entoun., vol. 1, p. 73.

1869. Larva, stages of. Harris. Entom. Corres., 1. 271, pl. 2 and 4.

1877. Larva, chrysalis. Freneh. Traus. Dejt. Agr. 111., vol. 15, 1. 138.

1880. Larva. Miss N. Middleton. Trans. Dept. Agr. 1ll., vol. 18, Apl., 1. 74.

1881. Larva, chrys. Gundlach. Entom. Cubaua, 1. 136.

1881. Clurysalis, (figs.) Sender. Intterflies, their strueture, ete., p.41, pl. (i).

1884. Larva. Dr. A. Gruber. Jena Zeitselır. Nat. Copied in Papilio, vol. 4, p. 87 .

1886. Larva, chrysalis. French. IButt. East. U. States, 1. 13.

Food Plaxts. Laurus, Sassufras.

Papilio palamedts. Irury.

1833. Larva. Boistulal-Leeonte. Hist. Lep. N. Amer., p. 17, pl. 5.

1836. Larva, chrys. Boisdural. Spee. (iener. Diurnie, vol. 1, p. 338.

1837. Larva. Weytuool, (quotes Bois.) Drury's Ill. Exot. Entom., vol. 1, p.' 36.

1861. Larva. Morris, (quotes Bois.-Lec.) Syuop. Lep. X. Amer., ๒. 7.

1864. Larva. Jaeger. Life N. Amer. Ins., p. 210.

1881. Lifo history. W. M. Edwarls. Canad, Entonı., vol. 13, p. 11!.

1886. Life hist., (condensed.) French. Butt. East. U. States, p. 9.5.

Food Plaxts. Laurus, Citrus.

Papilio turnus. L.

1833. Larva, (col'd fig.) Boisdure:-Leconte. IIist. Lep. X. Amer., p. 19. pl. 7.

1836. Larva, chrysalis. Boirdural. Spee. Gener. Diurni, vol. 1, p. 339.

1840. Larval stages, chrysalis. I. H. Gosse. Canarl. Naturalist, p]. 249, 293.

1856. Larva. Fiteh. 3i Rept. Ins. N. York, p. 341.

1857. Larva, chrysalis. Anonymons. Canad. Nat. and Geolog., vol. 2, p. 223.

1861. Larva. Morris, (quotes Bois.-Lec.) Synop. Lep. N. Amer., p. 2.

1862. Larva, chrysalis, (figs.) Harris. Ins. Inj. Vegetat., Flint's edit., 1. 268.

1863. Larva. B. D. Walsh. Proe. Entom. Soc. Pinila., vol. 1, p. 352.

18633. Chrysalis. I. A. Lintuer. Proc. Entom. Soc. Phila., vol. 3, 1. 51.

1869. Egg, larval stages. Suunders. Canad. Entom., vol. 1, 11. 51, 74.

1873. Life hist., (brief.) Saunders. Rept. Entom. Soc. Ontario, 1. 21.

1874. Life history. Saunders. Canad. Entom., vol, 6, 1. 2.

1876. Larva. G. H. Perkins. 2d Rejt. Vermont State Board Agric., 1. 589.

1877. Life hist., (col'd figs.) I. H. Elluarls. Butt. N. Autr., 2l series, pl. Picpilio, 3.

1877. Larva. Packard. Half-hours with Insects, 1. 180.

1877. Larva. Freneh. Trans. Dept. Agr. Ill., vol. 15, 1. 139.

1879. Larva, (figs., brief.) Saunders. Rept. Entom. Soc. Ontario, p. 73, fig. 4.

1880. Larva. Miss N. Middleton. Trans. Dept. Agr. IJl., vol. 18, [. 74.

1880. Larva. D. W. Coquillett, Trans. Dept. Agr. Mll., vol. 18, p. 173.

1883. Egg, larval stages, elirysalis (figs.) Saunders. Ins. Injur. Fruits, p. X3.

1884. Egg, larva, chrysalis (figs.) Ferwald. Butt. of Maine, 1. 24.

1881. Larva. Dr. A. Gruber. Jena Zeitschr. Nat. Copied in Papilio, vol. 4, p. 86.

1886. Life history, (figs. larva and chry8.) French. lintt. East. U.States, p. 100.

Food Plaxts. Prunus, Cevasus, Liriodendron.

Papilio turnus, dim. form, glaucus, $L$.

1833. Larva, chrysalis. Boisdural-Leconte. Hist. Lep. N. Amer., p. 22, pl. 9. 
1836. Larv, elıys. Boistural. Spec. Gener. Diurne, vol. 1, p. 336.

1856. Larva. Fitch. 3il Reyt. Inj. Ins. N. York, p. 376.

1859. Larva, (brief.) I. H. Gosse. Letters from Alabama, p. 123.

1877. Young larva, (col'sl tig.) W. IT. Edu'urls. Butt. N. Amer., 2d series, pl. Papilio, 4.

1881. Egg, (fig'll.) Scudder. IButtertlies, their strncture etc., p. T.

Papilio eurymedon. Bois.

1833. Larva, (col'l fig.) Titiun P'eale. Lep. Americanu, pl. 1.

1873. Larra, ehrysalie. Hy. Efluetrds. Proc. Cal. Acatl. Sc., vol. 5, 1, 164.

1874. Larva, chrysalis, (col'd tigs.) W. H. Fluarls. Butt. N. Amer, 2d series, pl. Papilio, 1.

Food Plavt. Rhamnus californicus.

Papil.o rutulus. Belv.

1870. Lurva, chrysalis. S. H. Scudder. Proc. Hoston Soc. Nat. Hist., vol. 19, p. 221. (Printerl as P. enrymedon in error.)

1873. Chrysalis. Hy. Edwards. Proc. Cal. Acad. Sc., vol. 5, p. 165.

1878. Larva. T. L. Meat. Psyehe, vol. 2, p. 180.

1882. Laval stages. Hy. Edtrurds. Papilio, vol. 2, p. 112.

1883. Larval stages. H. H. Eilwards. Papilio, vol. 3, p. 2.

188\%. Life history, (col'd figs.) W. H. Elluarly. Butt. N. Amer., 2d series, pl. $12,13$.

Food Plasts. Alnus riridis, Sulix sp.

Papilio daunus. Bdr.

1833. Larva, (eold figr.) Titian P'eale. Lep. Americana, pl. 1.

1873. Clirysalis. Hy. Edurards. Proe. Cal. Acad. Se., vol. 5, p. 325.

1883. Larva. W. II. Edworts. Papilio, vol. 3, p. 2.

1884. Larva. W. Scheus, jr. Papilio, vol. 4, p. 100.

Foov Puaxts, P'rumus, Cerasus.

Papllio pilumius. Bd $\mathrm{v}$.

1884. Larva, chrysalis. W. Scheus, jr. Papilio, vol. 4, p. 100.

Food Plaxt. " Feeds on Laurel."

Papilio cresphontes. Cram.

1833. Larra. -Boisdunal-Leconte. IIist. Lep. N. Amer., p. 31, pl. 12, 13, (c.s P. thoas.)

1836. Larra, chrys. Boistutul. Spec. Gener. Diumie, vol. 1, p. 356.

1859. Larra. P. M. Gosse, (‘notes Bois.) Letters from Alabama, p. 170, (as P. thoas.)

1862. Larra. Morris, (quotes Bois.-Lec.) Synop. Lep. N. Amer., 1. 8.

1877. Larra. G. H. French. Trans. Dept. Agr. Ill., vol. 15, p. 140.

1878. Larva, chrysalis. Suunders. Rept. Entom. Soc. Ontario, 1. 61.

1878. Larva. .J. Boll. Canad. Entom., vol. 10, p. 154.

1880. Larval stages. G. II French. 6th Ann. Rept. S. Ill. Nomal Univ., p. 43.

1880. Larva. Stumlers. Rept. Entom. Soc. Ontario, p. 41.

1880. Larva. W. H. Ashmead. Insects Injurious to the Orange, p. 47.

1880. Larva. Miss N. Midllleton. Trans. Dept. Agr. Ill., vol. 18, p. Tí.

1881. Larva, chrys. Gumllach. Entom. Cubana, p. 132.

1881. Life history. G. H. French. Canad. Entom., vol. 13, p. 17i.

1883. Egg, yonng larva. Suunlers. Canad. Entom., vol. 15, p. 234.

1883. Larva. A. I. Mundt. Papilio, vol. 3, p. 26.

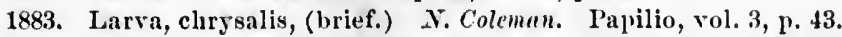

1883. Lifo hist., (brief) (figs.) Saunlers. Ins. Injur. to Fruits, p. 377.

1885. Larva, elurys., (col'd figs.) Hubjard. Ins. Affect. Orange, p. 137.

1886. Life historg, (condensel.) G. II. French. Butt. East. U. States, p. 103.

Food Plaxts. Plelea, Dietammus, Citrus, etc. 
Papilio polydamas. $L$.

1836. Larva. Boisdural. Spec. (iener. Jiurnu, vol. 1, p. 321.

186I. Larva. Morris, (quotes Boisduval.) Synop. Lep. N. Amer., p. 13.

1864. Larva. Jueger. Life N. Amer. Ins., 1. 210.

1878. Larva, chrys., (figs.) H. Deritz. Wiegemann Archiv. Naturgesch., p. 2 , fig. 1.

1878. Larva, chrys. Dexitz. Zitscl. gesament. natur.

1879. Larva, chrys. Devitz. Zeitsch. gesament. natur.

1881. Larva, chrys. Gundlach. Eutom. Cubana, p. 121.

Fool, Plant. Aristolochia.

Parnassius clodius. Men.

1879. Egg, young larva. W. H. Edwards, (as P. Baldur.) Canad. Entom., vol. 11, p. 142.

1885. Egg, young larva. T. H. Edwards. Canad. Entom., vol. 17, p. 64.

1886. Egg, larva. H.J. Eluees, (quotes Edw.) Proc. Zoül. Soc. London, Jan., p. 51.

Food Plants. Sedum, Saxifraga.

Parnassius smintheus. Dlyy. and Hew.

1872. Egg, (col'll figs.) W. H. Edwards. Butt. N. Amer., 1st series, pl. 3 of Paruassins.

1875. Egg, young larva. T. L. Meal. Rept. on Lep. of Wheeler Exped'n. Geolog. Survey, vol. 5, p. 743.

18i6. Egg, young larva. W. H. Edwards. Cauad. Entom., vol, 11, p. 141.

1883. Egg, larva. W. H. Eduards. Papilio, vol. 3, p. 158.

1885. Life history. Wr. H. Edwards. Canad. Entom., vol. 17, p. 61.

1886. Egg, young larva. $H . J$. Elues, (quotes Edw.) Psoc. Zoöl. Soc. London, p. 23.

Foon Plaxts. Sedum, Saxifraga.

Parnassius behrii. Edw.

1878. Larval stages. T. L. Mead. Psyche, vol. 2, p. 181.

Food Plant. Sclum.

Neophasia menapia. Felder.

1873. Chrysalis. Hy. Edwards. Proc. Cal. Acad. Sc. vol. 5, p. 165.

1882. Egg, Iarra. R. H. Stretch. Papilio, vol. 2, p. 103.

Food Prants. Pinus ponderosa, $P$. contorta, Abics balsamii.

Pieris monuste. L.

1833. Larva. Boisdural-Leconte. Hist. Lep. N. Amer., p. 43, pl. 16.

1836. Larva, chrys. Boisdurul. Spec. Gener. Diurnat, vol. 1, p. 497.

1861. Latra, chrysalis. Morris, (quotes Bois. and Lec.) Synop. Lep. N. Amer., p. 17.

1881. Larva, chrys. Gundlach. Entom. Cubaua, p. 101.

1883. Fgg, larva, chrys., (fig.) Riley. Rept. U. S. Dept, Agr., pl. 117-118, pl. 10, fig. 1.

1886. Egg, larva, chrysalis. G. H. French. Butt. East. U. States, p. 107.

Food Plants. Species of Crucifert.

Pieris beckeri. Edw.

1878. Larva, chrysalis. T. L. Mead. Psyche, vol. 2, p. 183.

1883. Life hist., (col'd figs.) W. H. Edwwards. Bntt. N. Amer., 2d series, pt. 11, pl. 1 of Pieris.

Food Plants. Varions species of Cruciferce.

Pieris sisymbrif. Bdv.

1878. Laira. T. L. Mead. Psyche, vol, 2, p. 184.

1883. Life hist., (col'd figs.) W. H. Edvards. Butt. N. Amer., 2d series, pt.11, pl. 1 of Pieris.

Food Plants. Species of Cruciferie.

Pieris protodice. Bois.-Lec.

1870. Life hist., (figs.) C. V. Riley. 2d Missouri Rept., p. 104.

1870. Larva, clirysalis, (fig'd.) Tounend Glover. Rept. U. S. Dept. Agr., p. 79. 
1870. Larva, chrys. C. I. Vinot. Amer. Eutom. vol. 2, y. 77.

1871. Larva, clirysalis, (fig'l.) Bethune. Rept. Entom. Soc. Ontario, p. $\mathbf{4 2 5 .}$

1873. Life hist. Saunders. Canad. Entom., vol. 5, p. 43.

1874. Larva, chrys. Hy. Educurds, (quotes C. I. Minot.) Proc. Cal. Acad. Sci., vol, 5, p. 327.

1877. Larva, chrysalis, (fig'll.) G. H. French. Trans. Dept. Agr. Ill., rol. 15, p. 141.

1877. Larva, chrysalis, (f.gs.) Packard. Inj. Ius. West, Hayden's Rept., l. io0.

1879. Larval stages, (figs.) Cyrus Thomas. Trans. Dept. Agr. Ill., vol. 17, Appertlix, p. 25.

1880. Larva, (fig'd.) Mfiss N. IIddleton. Trans. Dept. Agr. Ill., vol. 18, Ap. pendix, p. 77.

1880. Larva, (fig'd.) I. W. Coquillett, (quotes Riley.) 'Trans. Dept. Agr. Ill., vol. $18, \mathrm{p}, 178, A_{\text {plp. }}$.

1882. Larva, ehrysalis, (fig'll.) Scudder. Butt., their structure, etc., p. 21. .

1882. Larva. Suunders. Canad. Enton., vol. 14, p. 1.

1882. Larva, (fig'd.) Saunde's. Rept. Eutom. Soc. Ontario, p. 14.

1883. Egg, larva, clirys., (figs.) Riley. Rept. U.S. Dept. Agr., L!. 114-115; pl. 10 , tigs. 2-4.

1886. Life history, (condensed) (figs.) G. H. French. Butt. East. U. States, p. 109.

Food Plants. Species of Crucifor(r, especially Braszica.

Pieris napi. Esper.

1828. Larva, chrys. Stephens. Illust. Brit. Entom., Hanst., vol. 1, 1. 20.

1836. Larva, chrys. Boistueal. Spec. Gener. Diurnat, vol. 1, p. 519.

1859. Larva, ehrys. Edw. Neuman. Butt. Young England, p. 5.

1886. Life history, (figs.) Finch. Butt. East. L. States, 1. 11.

Food Plasts. Spccies of Crucifere.

Pieris oleracea. Harris.

1829. Larva. Harris. New England Farmer, vol. 7, 1. 402. (Eutom. Corres., p. 361, pl. 4.)

1841. Larva, chrys. Harris. Lus. Inj. Vegetat., lst edit., p. 214.

1850. Larva. Harris. Agassiz' Lake Superior, 1. 386.

1852. Life histors, (bricf.)' Harris. Ins. Inj. Vegetat., 2ll edit., p. 233.

185. Larra. Emmons. Natural Hist. N. York. Agriculture, vol. 5. p. 205.

1857. Egg, larra, (brief.) Anonymous. Canal. Nat. and Geolog., vol. 2. p. 347.

1862. Egg, larva, chrysalis. Harris Ins. Inj. Vegetat., Fint's edit., p. 270.

1864. Larra, chrysalis. Lintner. Proc. Entom. Soc. Plila, vol, 3, p. 54.

1869. Life history. Fitch. 13th Rept. Ins. X. Y., p. 531.

1869. Chrysalis, (fig.) Harris. Entom. Corres, pl. 4.

1870. Larva, ehrysalis, (fig'll.) Touneud Glorer. Rept. U. S. Dept. Agr., p. 79.

- 1870. Larva, chrysalis, (figs.) C. T. Riley. 2d Missouri Rept., p. 105.

1871. Larva, chrysalis, (figs.) Bethune. Rept. Futom. Soe. Ontario, p. 424.

1873. Life history, (fig'd.) Saunders. Canad. Entom., vol. 5, p. 37.

1877. Egg, larva, (figs.) Packurd. Inj. Ins. West, Hayrlen's Rept., p. $7+6$.

1877. Larva, (fig'd.) Packard. Half Hours with Insects, p. 52.

1877. Larva. French. Trans. Dept. Agr. Ill., vol. 15, p. 143.

1880. Larva, (fig't.) Miss N. Mildleton. Trans. Dept. Agr. Ill., vol. 18, p. 77, A pp.

1881. Fgg, elirysalis, (figs.) Scuhder, (after Riley.) Butterflies, their structure, etc., p. 165.

1883. Egg, larva, (fig'l.) Riley. U. S. Agr. Rept., p. 108, pl1.1.

1884. Egg, larra, chrysalis, (figs.) Fernald. Butt. of Maine, 1. 25.

Food Plasts. Speeies of Cruciferer.

Pieris rapæ. L.

1828. Larva, chrys., (hrief.) Stephens. IIl. Brit. Entom., Haust., vol. 1, p. 19.

1836. Larva, chrys. b lucul. Spec. Gener. Diurua, vol 1, p. 520.

1837. Larva, chrys. Kollar. Inj. Insects (London's edit.), p. 150.

1842. Larva, chrys., (figs.) Curtis. Jour. Royal Agr. Soc. England, p.: 312.

1856. Larva, chrys. Chenu-Lucas. Encyc. Natur. Papillons, p. 50. 
1859. Larva, ehrys. Edw. Nereman. Butt. Young Englaud, p. 5.

1869. Lifu history. Fitch. 13th Rept. Ins. N. York, p. 543.

1870. Larva, ehrys., (tigs.) T. Glover. Rept. U. S. Dept. Agr., p. 78, tig. 38.

18i0. Life history, (tigs.) C. V. Riley. 2d Missonri Rept., p. 106.

1871. Larva, (fig.) Bethune. Rept. Entom. Soc. Ontario, p. 423.

1872. Larva, chrysalis, (figs.) Townend Glores. Rept. Enton. N. York State :gr. Assoc., p. 82, tig. 15.

1873. Larra, (fig'd.) A. J. Cook. 12th Rept. Michigan Board Agr., 1. 120, fig. 16.

1873. Life history. Suunders. Canad. Entom., vol. 5, 1. 42.

1875. Egg, larva, ehrysalis, (figs.) Saunders. Rept. Eutou. Soc. Ontario, p. 3 I.

1877. Larva, chrysalis. Fieneh. Trans. Dept. Agr. 1ll., vol. 15, p. 145.

1877. Larva, chrysalis, (figs.) Paekarl. Inj. Ins. West, Hayden's Rept., p. 747.

- 1877. Larva, (fig'd.) P'ackard. Half Hours with Inseets, p. 54.

1878. Egg, larva, ebrysalis, (fig'll.) J. Stauffer. Agr. Peunsylv.

1879. Larva, (fig'd.) Miram Cuttiny. Pests of Farmer, Now Hampshire Board Agr., p. 34.

1879. Larval stages. Cyrns Thomas, (quotes Curtis and others.) 'Tians. Dept. Agr. Ill., vol. 17, pp. 10-25.

1880. Larva, ehrysalis, (tig'd.) Miss N. M:ddleton. 'Trans. Dept. Agr. Ill., vol. 18, p. $77, \mathrm{App}$.

1880. Larva, (fig.) D. W. Coquillett. Trans. Dept. Agr. Ill., vol. 18, p. 179, App.

1881. Larva, chrysalis, (fig'd.) Scudder, (after Riley.) Butt., their structi:re, etc., p. 124, fig. 112.

1882. Larva and chrysalis. Saunders. Canad. Eutom., vol. 1., p. 2.

1882. Larva, chrysalis, (hrief.) W.F. Kirby. European lutt. aud Moths, p. 8.

1883. Egg, larva, elirys., (figs.) Riley. Rept. U S. Dept. Agr., 1p. 108-113, pl. 1 , tigs. 1-lt.

1884. Egg, larva, chrysalis. Fernald. Butt. of Maine, p. 27.

1885. Clirysalis, (fig.) Ify. Edwurds. Standarl Nit. Hist., rol. 2, p. 495.

1886. Life history, (figs.) French. Butt. East. U. States, p. 115.

1887. Larva. J. Fletcher. Rept. Ent. Dept. Agr. Cauadu, p. 16.

1888. Larva, (brief.) J. F'letcher. Rept. Ent. Bot. Dept. Agr. Canada, 1. 16.

Food Plasts. Species of Crueiferer.

Pieris occidentalis: Reakt.

1888. Chrysalis. Hy. Edicarils. Entom. Amer., vol. 3, p. 162.

Anthocharis lanceolata. Bdv.

1878. Larva. T. L. Mcal. Psyche, vol. 2, p. 183.

Food Plast. Turritis glabra.

Anthocharis ausoniedes, Bilv.

1874. Larva. Hy. Eilwarlds, (quotes 'T. L. Meal in lit.) Proc. Cal. Acad. Sc., vol. 5, p. 326.

1874. Egg, larva, chrysalis, (col'd figs.) W. H. Edwards, (quotes T. L. Head in lit.) Butt. N. Amer., 2ll series, pl. 2 of Anthocharis.

1885. Larva, chrysalis. W. H. Ellwards. Butt. of N. Amer., 2l series, pl. 13.

Food Plants. Species of Cruciferr.

Anthocharis hyantis. Edw.

1878. Larva. T. L. Meaul. Psyche, vol. 2, p. 183.

Callidryas eubule. L.

1797. Larva, (col'd fig.) Abbot-Smith. Lep. Ins. Georgia, pl. 5.

1833. Larva, (col'd fig.) Boisduval-Leconte. Hist. Lep. N. Amer., p. 74, pl. 24.

1836. Larva, chrysalis, (col'd figs.) Boisdural. Spec. Gener. Diurna, vol. 1, pp. $614-615, \mathrm{pl} .2$.

1842. Larva, (col'd fig.) Dunean. Natural. Libr., vol. 21, p. 123.

1862. Larva. Morris, (quotes Boisd.) Syuop. Lep. N. Amer., p. 26.

1873. Larva, (brief.) Butler, (quotes Abb.-Sin.) Monogr. Callidryas, p. 14.

1881. Larva, chrysalis. Gundlach. Entow. Cubana, p. 115. 
Foon Plant. Cassia.

Callidryas sennæ. L.

1725. Larva, chrysalis. Sloan. IIist. Jamaicn, p. 212.

1832. Larva, clirysalis, (col'd figs.) Poey. Cent. Lep. Cuinana.

1855. Larva, clirysalis. Sepp. Suriuamsche Vluiders, 1, p. 19, (пs Pap. IIyperici.)

1873. Larva, chrysalis. Butler, (quotes Sloan.) Monogr. Callidryas, p. 17.

1873. Larva, chrsualis. Butler, (quotes Sepp.) Monogr. Callidryas, p. 16.

1886. Larva, (brief.) Frenci. Hutt. East. U. States, 1. 123.

Foon Prant, Cassia.

Callidryas philea. L.

1873. Larva, chrysalis, (brief.) Butler. Monogr. Callidryas, p. 22.

1888. Clirysalis. Hy. Edwarls. Entom. Amer., vol. 3, p. 162.

Colias eurydice. Bdv.

1876. Larva, chrysalis. Hy. Ellwarls. Proc. Cal. Acad. Sc., vol. 7, p. 61.

1883. Life history. W. H. Edwarls. Canad. Entom., vol. 15, p. 224.

Food Plast. Amorpha californica.

Colias cæsonia. Stoll.

1833. Larva. Boisiural-Leconte. Hist. Lep. N. Amer., p. 67, pl. 22.

1836. Larva. Boisduval. Spec. Gener. Diıruse, vol. 1, p. 636.

1862. Larva. Morris, (quotes Bois.-Lec.) Synop. Lep. X. Amer., p. 27.

1874. Larva. Hy. Eilwards, (quotes Boisduval.) Proc. Cal. Acad. Sc., rol. 5, p. 327.

1880. Larva. Miss $N$. Middleton. 'Traus. Dept. Agr. Ill., vol. 18, p. 78, App.

1881. Larva, chrysalis. Gundluch. Entom. Cubana, p. 106.

1886. Larva, (brief.) French. Butt. East. U. States, p. 128.

1888. Life history. W. H. Edwarls. Canad. Entom., vol. 20, p. 21.

Foon Plasts. Trifolium, Amorpha.

Colias meadii. Edw.

1839. Life history. $\Pi$. H. Elwarls. Canad. Entom., vol. 21, 1. 41.

Colias eurytheme. Bdv.

1869. Egg, larva, chrysalis. ' W. H. Elwards; (†notes J. K. Hayhurst.) Butt. N. Amer., 2d series, Colias No. 3.

1872. Egg, larva, chrysalis. W. H. Eduards. Supplem. Notes to Butt. N. Amer.

1878. Egg, larval stages, (figs.) W. H. Edwards. Butt. N. Amer., 2d series, Colias No. 4.

1880. Larva. Miss N. Middleton. Trans. Dept. Agr. Ill., vol. 18, p. 78, App.

1886. Life history, (condensed.) G. H. Fvench. Butt. East. U. States, p. 131.

Food Plant. Trifolium.

Colias hagenii. Edw.

1883. Life history. W. H. Elwards. Papilio, vol. 3, p. 163.

Food Prast. Trifolium.

Colias philodice. Godt.

1857. Larra, (brief). Anonymous. Canad. Nat. and Geolog., vol. 2, p. 318.

1862. Layva, chrysalis, (fig'd.) Harris. Ins. Inj. Vegetat., Flint's edit., p. 272.

1869. Egg. Saunders. Canad. Entom., vol. 1, p. 54.

1870. Larva. Riley. 2d Missouri Rept., p. 116.

1870. Larra, chrysalis. Saunder's. Canad. Entom., vol. 1, p. 55.

1873. Larva, chrysalis. Saunders. Canad. Entom., vol. 5, p. 222.

1874. Egg, larva, chrysalis. Saundyrs. Rept. Entom. Soc. Ontario, p. 27.

1876. Life history, (col'd figs.) W. H. Edwards. Butt.N. Amer., 2d series, Colias No. 3.

1880. Larva. Miss N. Middleton. Trans. Dept. Agr. Ill., vol. 18, p. 78, Apl.

1881. Egg, larva, chrysalis, (figs.) Sculder. Butt., their structure, etc., pp. 8, $42,116$.

1882. Life history, (brief.) Saunders. 12th Rept. Entom. Soc. Ontario, p. 48. 
1881. Eugr, larva, chrysalis. Neruald. 13utt. of Maine, p. 31.

1886. Lite history, (coudensed.) Frrench. Butt. Wust. U. Sitates, p. 1:31.

Foon P's. INrs. Trifolinm, dytroyglus.

Colias scudderii. Reak.

1372. Young larva. W. M. Eiluarly. Butt. N. Amer, Colias, No. 8.

Colias alexandra. Eilw.

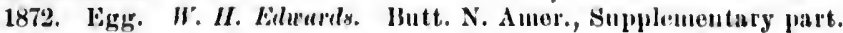

Colias harfordii. (iuel. C. Barloara \&.)

1881. Lilio history. W. II. Vidwerels. Pappilio, vol. 1, 1.2.

Foon Plant. Antrayalus crotaluria.

Terias nicippe. Craun.

1833. Larva, (col'd tig.) Tition l'cule. Lep. Amer., pl. $\not$.

1833. Larva. Boistmeal-Leconte. II ist. Lep. N. Amol, p. 5n, pl. 20.

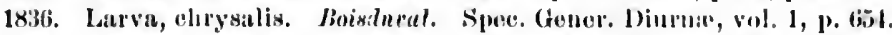

18\%5. Larva, eluysulis. Toumenl Glortr. Traus. N. York State Agr. Soo, p. 105.

18̈̈). Larva, ehrysalis. Chemu-Lures. Eutom. Nat. Hist. l'apillons, p. (i).

1862. Larva, ehrysalis. Morris, (quotes Bois.-Loc.) Synop. Lep. N. Amer., p. 33.

1873. Chrysalio. My. Liduruds, (quotes Bois.-Lee.) I’roc. Cal. Aeud. Se. vol. 5, 1. 166.

1880. Latra. Miss N. Millllefon, (quotes Bois.-Lee.) 'Trans. Dept. Agr. III., vol. 18, p. 79, $\Lambda_{\mathrm{p} p}$

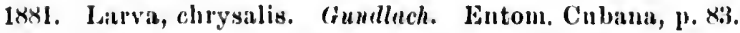

18x1. Life history. W. H. Eiluerils. Canal. Enton., vol. 13, p. 61.

1881. Chrysalis. Scudles. Butt., their structure, etc., p. 181.

1886. Larva, ehrysalis. French. Butt. East. U. States, p. 137.

Foon, liant. Cassia.

Terias lisa. Bilr.

1833. Larva, ehrysalis, (col'd fig.) Bois.-Lec. IIist.-1.ep. N. Amer., p. 53, pl. 19.

183t. Larva, elurysalis, (eol'd tig.) Boisduval. Spec. Gener. Rhopaloc., vol. 1, pl. $2,1,662$.

1862. Larva. Morris. Synop. Lep. N. Amer., p. 31.

1874. Larva, chrys, (1,rief.) $M_{y}$. Edwarly, (1notes Boisdnval.) I'roe. Cal. Acall. Sc., vol. 5, p. 327.

1880. Larva. Miss Middleton, (quotes Boisd.) 'Trans. Dept. Agr. Ill., vol. 18, p. 79, Append.

1886. Larva, (briof.) French. Butt. East. U. States, p. 139.

Food l'Lants. Cassia, Trifolium.

Terias delia. Cram.

1883. Larva. Bois.-Lec. Itist. Lep. N. Amer., p. 49, pl. 18.

1836. Larva, chrysalis. Boistlueal. Spec. Goner. Diurna, vol. 1, p. 661.

1862. Larva. Morris, ('nnotes bois.) Synop. Lep. N. Amer., p. 35.

1874. Larva, chrys, (briof.) Hy. Edwarils, (quotes Boisduval.) P’roc. Cal. Acid. Se., vol. 5, 1. 327.

1886. Larva, (brief.) French. Butt. East. U. States, p. 140.

Food Plants. Cassia, Trifolium.

Heliconiá charitonia. L.

1850. Larva. P. H. Gosse. Note in Doubleday. Gen. Diur. Lep., vol. 1, p. 97.

1879. Larva, elırysalis. Dewitz. Zoitseh. gesument. natur.

1881. Larva. W. H. Ellwards. Papilio, vol. 1, p. 209.

1831. Larva. W. H. Edward8. Canad. Entom., vol. 13, p. 158.

1881. Larva, ehrysalis. Gundlach. Entom. Cubana p. 21.

1882. Life history. W. I. Edwards. Butt. N. Amer., 2l series, part 10.

1886. Life history, (eonlensod.) French. Butt. East. U. States, p. 142.

Food Plant. P'arsiftorti.

BULL. 35-2 


\section{Danais archippus. Latr.}

1797. Larva, chrysalis, (col'd thgs.) Ahlot-Smith. Lep. Ius. (ieorgia, pl. ti.

18:8. Larva, ehrys., (llig.) Suy. Amer. lintom., vol, 3, pl, 5l.

18:33. Larva, (col'd figs.) T. I'etele. Lop. Americana, pl. 7.

18:\$3. Litrva, chrys., (col'd figs.) Bois,-Lec. Hist. Lep. N. Amer., 1. 137, pl.40.

1810. Lan'in, chryн., (ligs.) Wreturood. Intr. Mod. Class. Ins., vol. 2, 1. 352

1851. Iarva, chrys., (col'd tigs.) Eimmons. Nat. Hist. N. York, vol. 5, pl. 38.

18.,7. Inrva, chrys., (lig.) Anon. Canad. Nat. nul Geol, vol, 2, p. 351.

18,9. Latra, ehrys. I'. H. Gosas. Letters from Alahama, p. 185.

1862. Larva, chrys. Morrin, (quotos Bois.-Lec.) Synop. Lup. N. Amer., p. 38.

186\%. Larvil, pupa. Iluris. Ins. Inj. Vegetnt., Flint's edit., p. 280.

186\%. Larva, chrysulis. Saunlers. Canal. Entom., vol. 1, p. 75.

1870. Egg, chrysalis. Saunlers. Canal. Entom., vol. 2, p. 118.

18il. Lifo history, (tigs.) Rilcy, 3i Missonri Rept., p. 1.l.

1873. Larra, ehrysalis. My. Eilururds. Proc. Cal. Acal. Se, vol. 5, 1. 166.

1873. Life history. Saunlers. Canad. Entom., vol. 5, p. 4.

1876. Lifo history, (bricf.) W. M. Eilicardle. Canal. Entom., vol. 8, p. 119.

18i8. Lifo history. W. M. Erlirards. Payche, vol. 2, p. 170.

18ix. Larva, ehrys. H. Deritz. Wiegemann Archiv, Naturgesch., p. 9, pl. 1.

18ī8. Larva, chrysalis. Dewitz. Zeitsch. gesament. uatur.

1879. Larva, ehrys., (figs.) Mrs. I. I'. Ballath. Insect Lives, p. 24.

1879. Larva, chrys. Deuitz. Zoitseh. gesament. natur.

1880. Larva, (tig.) D. Coquillett. 'Trans. Dept. Agr. Ill., vol. 18, Append., p. 161.

1880. Larva, (fig.) Miss Midilleton. 'Trans. Dept. Agr. Ill., vol. 18, A plpend., p. 80.

1881. Larva, chrys. Gunllach. Entom. Cubana, p. 23.

1881. Egg, larva, (figs.) Scudiler. Butt., their structure, utc., [1). 10, 18, ligs. $17,18,22$.

1881. Chrysalis, (fig.) Scuclder. Loc. cit., p. 4t, fig. 61.

1881. Eggr, larva, chrysalis, (ligs.) Feruald. Bntt. Maine, 1) 35.

1885. Larva, chrys. My. Eiluards. Kingsloy's Stand. Nat. llist., vol. 2, p. 492.

1886. Lifo history, (condonsed,) (ligs.) French. Butt. Last. U. States, p. 144.

1888. Chrysalis, (fig.) Grote. 18th Rept. Entom. Soc. Ontario, p. 74.

1888. Larval stages, (fig.) Lintuer. Ith Rept. N. Y. Stato Entom., p. 136.

Food Plant. dsclepias.

Danais berenice. Cram.

1833. Larva, chrys., (figs.) Bois,-Lcc. Hist. Lep. N. Amer., p. 131, pl. 39.

1862. Larva, chrys. Morris, (quotes Bois.-Lec.) Synop. N. Amer. Lep., p. 37.

1874. Larva, elnys. My. Eiliards, (quotes Boisduval.) Proc. Cal. Acad. Sc., vol. 5, p. 327.

1881. Larva, chrys. Gundlach. Entom. Cubana, p. 25.

1886. Larva, chrys. French. Butt. East. U. States, p. 146

Fool P'lants. Nerium, Asclepias.

Agraulis vanillæ. Cr.

1769. Larvil, ehrys. Abbot-Smith. Lop. Ins. Georgia, pl. 12.

1833. Larva, chrys., (ligs.) Bois.-Lec. Itist. Lop. N. Amor., p. 143, pl. 42.

1955. Latra, chrysalis. Townend Glover. Trans. N. York State Agr. Soc, p. 106.

1862. Larva, chrysalis. Morris, (quotes Bois.-Lec.) Synop. Lop.y. Amor., pp. $40,41$.

1874. Larya, clirys. Hy. Eilwarls, (quotes Boisduval.) Proc. Cal. Acad. Sc., vol. 5, p. 327.

1880. Larva. Miss Middleton. Trans. Dept. Agr. Ill., vol. 18, $\Lambda$ pp , p. 80.

1880. Life history. W. H. Edwards. Canad. Entom., vol. 12, p. 122.

1881. Larva, chrys. Gundlach. Eutom. Cubana, p. 57.

1886. Lifo history, (condensed.) Freuch. Butt. East. U. States, p. 148.

Foon Plant. Passiftora. 
t).

\section{9.}

l. 1.

1. 11 it. , 1. 80 . s, ligs.

Aroyunis, idalia. Drury.

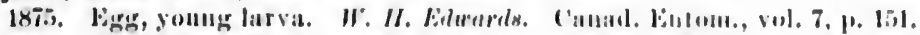

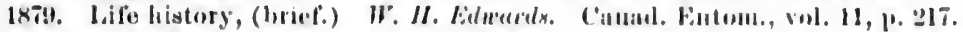

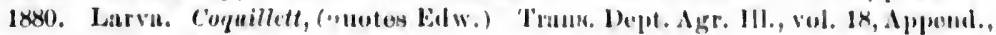
p. 162.

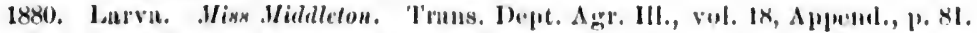

1881. Fojg, larva. Fermeld. lintt. Maine, p. 37.

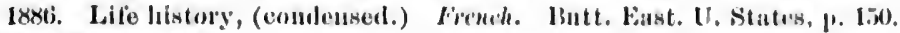

Argynnis diana. Crati.

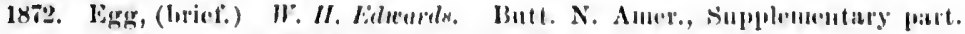

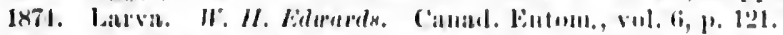

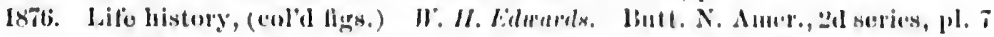
of Argyouis.

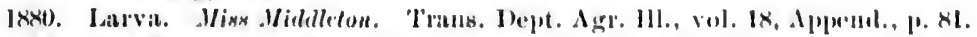

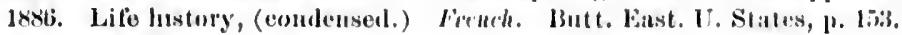

Aroynnis cybeie. $\mathrm{F}$.

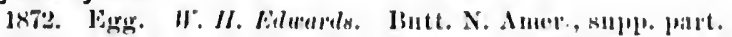

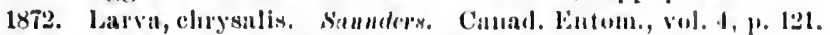

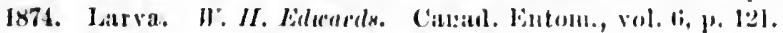

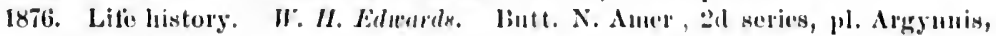
Ni) 7.

1880. Larva. Mins Middleton. 'Trams. Dept. Agl. III., wol. 1x, .1ppentl., p. 81.

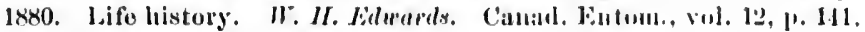

1886. Lilu listory, (comdensed.) Firench. Butt. Jast. I'. States, 1. 155.

Argynnis aphrodite. F.

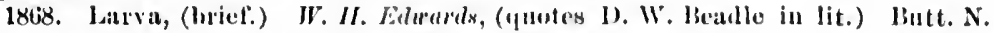
Amer., Ist scries, pli. Arerymis, No. :3.

1874. Lallva. W. IT. Edeards. Camad. Esitum., vol. 1;, p. 121.

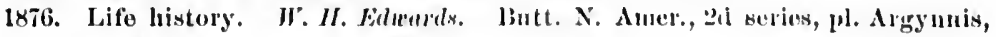
No. 7 .

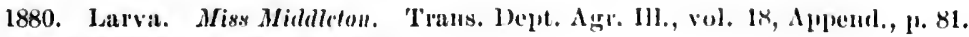

Argynnis hesperis. W. H. Eilwards.

1888. Life history. II. II. Eillearls. Cinald. Eutom., vol. 201), 1. 17.

Argynnis edwardsii. Reakirt.

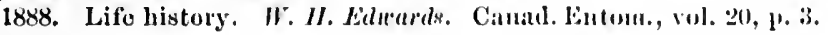

Argynnis alcestis. Edw.

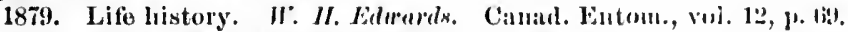

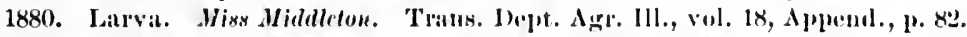

1886. Lifo history. Fromch. Butt. Gast. U. Stiltes, 1. 159.

Argynnis atlantis. Edw.

1868. Larvis. (brief.) W. M. Elwards, (quotes C. II. Shuthleft in lit.) Butt. N. Amer., pl. Árgynuis, No. 5.

1869. Larva, chrysalis. Powletrol. Guide to study of lus., p. 2i2.

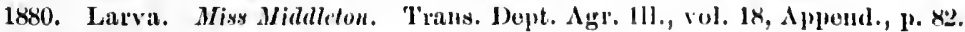

1881. Chrysulis, (fig.) Scudler. Butt., their structure, ote., p. 150.

1886. Chrysalis, (fig.) French. Iutt. East. U. States, p. 1ti!.

1888. Lifo history. IF. II. Edtuarly. Canul. Finton., vol. 20, p. 3.

Argynnis eurynome. Edw.

1875. Egg, (col'd fig.) W. M. Eillcards. Butt. N. Awer., Qd series, pl. Argyuuis, No. 1.

Argynnis egleis. Bois.

1879. Larva. W. H. Edwards. Cinal. Entom., vol, 11, భ. 53.

1879. Lifo history. W. II. Eidwarls. Canal. Entom., vol. 11, p. 177.

1880, Larva. D. W. Coquillett, (quotes Edwards.) 'Trans. Dept. Agr. Ill., vwl. 18, Append., p. 161. 
Argynnis myrina. C'ran.

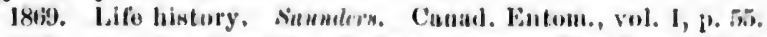

166\%. Larva, chrysulis. I'nckerril, (funtes samuders.) linides to sturly of Ins., 1. 254.

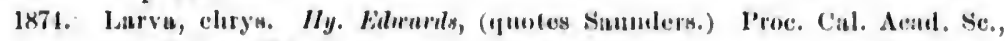
vol. $5,1.328$.

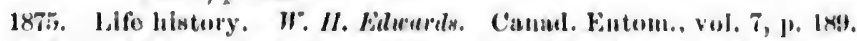

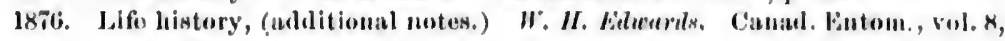
1. 161 .

18*0. Larva. Mi*a Mihllleton. 'Trans. Dept. Agr. Ill., vol. 1X, Append., p. Xะ.

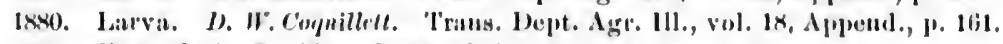

18xI. ligg, (fig.) Scudldr. Butt., their ntruefure, cte., p. $x$.

18x1. Larval stages, (lig.) Cirnber. Jena Zeitachl. Nat., eopied in Inapilio, vol. I, p. 91.

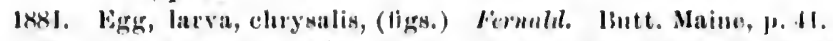

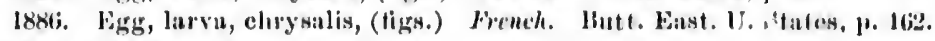

Argynnis montinus. Sent.

1871. I,ife libtory, (figs.) Sculler. (ieology N. Hampsl., pl. I.

Argynis bellona. Fal..

1871. Ligg. Scmelicr. Rept. l'enbody Acull. Sc., p. 19.

1872. Lifo history. Sculdrr. Amer. Nuturalist, vol. 6, p. 513.

1880. lifo history, (noten on.) W. II. Lillewids. P'apilio, vol. 1, 1. 1:H.

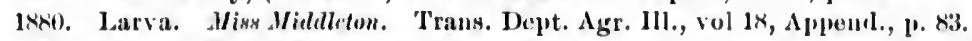

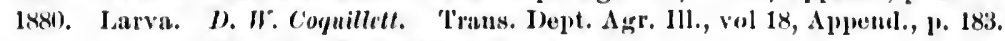

1881. Larva. Firnuld. Butt. Maine, p. 41.

1888. Clarysalis. Hy. Edarurty. Entour. Amer, vol. 3, 1. 162.

Foom P'saxis (of all the npecies of Argynnis). Viola of varions npecies.

Euptoieta ciaudia. Cran.

1833. Lar'a, elirysallis, (figs.) Bois.-Lec. Hist. Lep. N. Amer., p. 153, pl. 11.

1855. Larva, elirysulis. Tournend Glover. Traus. N. Y. Stato Agr. Soc., 1. 10:5.

16ii2. Larva, elirysalis. Morrix, (‘uotes Bois.-Lec.) Synop. Lep. N. Amor., 1. At.

1870. Larva. W. H. Edwarls. Canad. Entom., vol. 2, 1. 163.

1875. Eggg, larva. T. L. Meat. Rept. Leppil. Wheeler Survey, vol. 5, 1. 7:1.1.

1880. Larva. Miss Midlllton. Trans. Dept. Agr. IIl., vol. 18, Append., 1. 80.

1880. I.ifo history. W. II. Eilueurls. Caual. Eutum., vol. 12, j. 231.

1881. Lawa, chrysalis. Fermall, (quotes W. H. Edwards.) Hutt. Maine, 1) 43.

1886. Life history, (brief.) French. Hutt. East. U. States, 1. 16ib.

Foon, P'daxts. Tiola, Prasiflora, Sedum, Desmodium, Porlulaca.

Melitæa phaeton. Drnry.

1868. Larva, chrysalis, (figs.) Puckurl. Amer. Natumalist, vol. 2, p. 219.

1869. Egg, larval stages. Lintner. 23d Rept. N. York State Cab. Nat. Hist., pp. $154-15 \pi$.

1871. Ligg. Scudder. Rept. Penbody Aeal. Se., p. 51.

1875. Life history. W. MI. Ethurils. (Ganal. Eutoul, vol. 7, p. 150.

1875. Life history, (col'd figs.) I'. IT. Edlwerls. Butt. N. Amer., 2d series, pl. Melitaea, No. 1.

1877. Larva. Scudler. 1'syche, vol. 1, p. 18.

1877. Larva, elirysalis, (col'd fig.) P'ackarel. Half hours with Luseets.

1880. Larva. Miss Milllleton. 'Trans. Dept. Agr. Mll., vol. 18, Append., p. 83.

1E81. ligg, (fig.) Scudder. Butt., their strueture ote., p. 9.

1881. Web and nest. Scudler, (quotes Edwarls.) Butt., their structure, ete., p. 106.

1881. Larva-notes ou. W. II. Ellwartls. Papilio, vol. 4, p. 68.

1884. Larval stages, (figs.) (iruber. Jena \%eitsehr. Nat., p. 17, pl. 8.

188.I. Larval stages, (figs.) Gruber. Loe. eit. eopied in Papilio, vol. 4, 1. 87.

1884. Egg, larva, ehrysalis, (tig.) Feruuld, Butt. Maine, p. 44. 


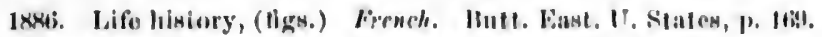

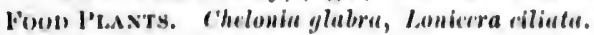

Melitaa cooperi. Ihelır.

1863. Larva. Behr. Proc, (a). Aeml. sc., val, 3, p. 90,

Melitae chalcedion. Doulb.-Hew.

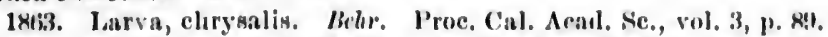

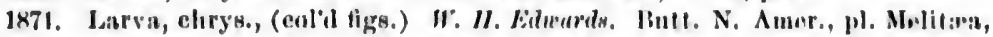
No. 1.

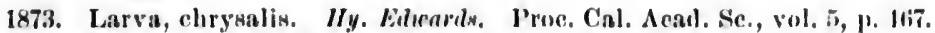

18x3. Figg, larvin. W', G. Wright. l'apilio, vol. 3, p. 1293.

1881. Larva an! partial history. W. II. Billurils. Papilio, vol. 1, p. 6i3.

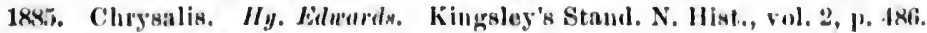

Foon Plaxts. Mimulun, Custiliju.

Melitæa nubigena. Bohr.

1875. Larva, chrysalis. T. L. Meral. Rept. Lep. Wheler Survey, vol. 5, p. 7ix.

Melitæa baroni. Hy. Ellw.

1879. Larval stages. W. II. Eiduards. Canst. Jintom., vol. 11, p. $11 !$.

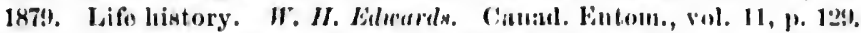

1880. Larva. Coquillett, („quotens E,dwarils.) 'Traus. Dept. Agr. III., vol. IR, dppent., p. 163.

Foov Plasts. Species of Sivophularincer.

Melitæa rubicunda. $11 y$. Ellw.

1885. Larval stages. W. II. Eilleards. Cautul. Eutour., vol. 17, p. 1\%.

Foon Puant. Serophularia.

Melitxa editha. Bois.

1873. Larva, chrysalis. Hy. Eilueards. Proc. Cal. Acarl. Sc., vol. 5. p. Ili.

Foon Plaxte. Erodium cicutarium, Trifolium, Viola.

Melitan palla. Bois.

1873. Larva, clırysalis. Hy. Edurards. P'roc, Cal. Acad. Sc., vol. 5, p. 16it.

Foon Plast. Castuldijia brcuiflora.

Melitaca harrisii. Scuil.

1877. Lifo history. W. II. Edwarits. Canad. Entom., vol. !, p. 16i.

1877. Larva. Sculler. P'sycho, vol. 1, p. 18.

1880. Larva. Coquilletl, (quotes Ellwards.) Trans. Dept. Agr. Ill., vol. 18, $\Lambda$ ppend., p. 162.

1881. Chrysalis, (fig.) Sculder. Bntt., their structure, ete., p. 32.

1881. Lifo history. French. Butt. East. U. States, p. 170.

188!. Larra, clirysalis, (figs.) French. Butt. East. U. States, p. 170.

Foon Plant. Diplopappus umbellatus.

Melitæa minuta. Edw.

1879. Chrysalis. 'W. II. Edwards. Canal. Eutom., vol. 11, p. 111.

Melitæa ismeria. Bois.-Lec.

Nork.--There is still some doubt as + this species. I therefore give the refercuces to its early stnges.

1833. Larva, chrysalis, (figs.) Bois.-Lec. IList. Lep. N. Amer., p. 168, Yl. 46.

1862. Larva, chrysalis. Morris, ('unotes Bois.-Lec.) Synop. Iep. N. Am., p. 50.

Phyciodes nycteis. Doub.-Hew.

1869. Life history, (figs.) Lintner. 23d Rept. N. York State Cal. N. Ilist., p. 158, pl. 8.

1870. Larva. W. H. Edwards, (as P. Harrisii.) Canal. Entom., vol. 2, p. 163.

1872. Egg. Salunders, (as M. ITarrisii.) Canad. Entom., vol. 4, p. 161.

1872. Larval stages. Saunders, (as M. Harrisii.) Loc, eit., p. 162.

1872. Larva. Lintuer. Intom. Contril., No. 1, p. 26.

1873. Larva. W. H. Edwards. Canal. Eutom., vol. 5, p. 224.

1874. Larva, chrysalis. Kiley. Proo. Amer. Ass. Adv. Sc., p. 108.

1875. Larva, chrysalis. W. II. Edwards. Canal. Entom., vol. 7, p. 151. 


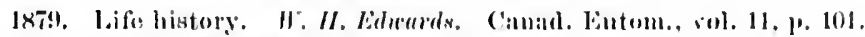

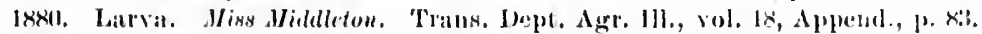

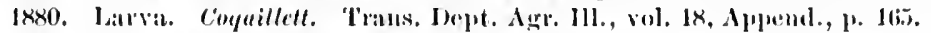

1884. Lifo history. Graber. Jena \%eitsehr. Nat., vol. 17, p. Aix, pl. 8. Copied in l'apilio, vol. 4, p. !0.

188.1. Life history, (brief.) Ferwall. Butt. Maine, p. If.

18x6. Lifo history, (eondensed.) Firench. Butt. Last. U. States, p. 172.

Foon Plasts. Species of Helienthus and telinomerix.

Phyciodes vesta. Filw.

1879. Lifo history. W. II. Edurards. Camad. Entom., vol. 2, p. 129.

Phyciodes camililus. lilw.

188. Lifo history. II. II. Edtemerls. Papilio, nol. 4, l. 128.

Foon Plaxt. Aster of varions nquecies.

Phyciodes tharos. Drury. (incl. vits.)

1862. Chrysalis. Marris. Ins. Inj. Vegetat., Flint's ed., p. 290.

1875. Life history. T. L. Mcull. Canaul. Entom., vol. 7, p. 1 li1.

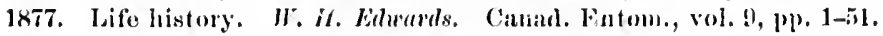

1878. Lifo history, (col'il figs.) II. II. Eidadals. Butt. N. Aner., 2d series, pl. Pliycioder, No. 1.

1878. Life history, (figs.) Gruber. Jena Zeitschr. Nut., vol. 17, 1, 17i, pl. 8.

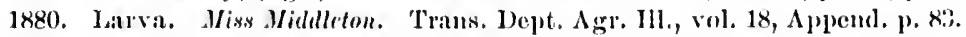

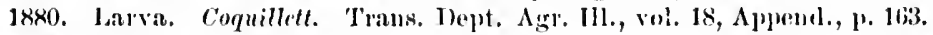

1881. Lat val stagres. Gruber. Jena Zeitschr. Nat., vol. 17, coppied in l'apilio, vol. 1 , ple. 90-120.

1884. Lugg, larva. Firmold. Butt. Maine, p. 18.

1886. Life nistory, (eondensed.) Freneh. Butt. East. U. States, 1. 17x.

Foon P'Aaxt. Aetingmeris helianthoides.

Phyciodes mylitta. Edw.

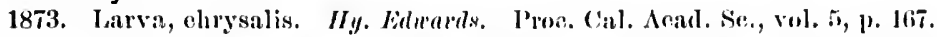

1875. Larva, ehrysalis. $T$. '. Mead, (gnotes IIy, Edwards.) Rept. Lep. Wheeler Expd., vol. v. 76.4 .

Foon Plasts. Cardums (thislle ol various spreeies.

Phyciodes picta. Edw.

1884. Life history. W. M. Eiduratls. Cumat. Entom., vol. 16., p. 163.

Eresia texana. Elw.

1879. Larval stages, chrysalis. IV. II. Edwards. Canal. Entom. vol. 11, p. 127.

1880. Larva. Coquilletl, (funtes Edwards.) 'Trans. Dept. Agrr. Ill., vol. 18, $A_{\text {plpeudl., p. } 164 .}$

Synchloe (Coatlantona) janais. J)rury.

188:3. Larva, chrysalis. W. Schaur, jr. Papilio, vol. 8, p. 188.

188x. Chrysalis. IIy. Edwards. Eutoni. Auner., vol. 3, p. 161.

Grapta interrogationis. Fatl. (inelucl. vars.)

1833. Larva, elırysalis, (figs.) Bois.-Lec., (as V. e-aurejmm.) Ilist. Lupiu. N. Amer., p. 192, pli. 51.

1810. Larva, elrysalis. P. il. Gosse. Canal. Naturalist, p. 280, (as (1. a -il11reum.)

1811. Larva, ehrysalis. Itarris. Inj. Ins. Vogetat., 1st celit., 1. 220.

18:2. Larvi, clurysalis. Ifarris. Inj. Ins. Vegetat., 2 l edit., p. 210.

185.4. Larva, (col'd fig.) Emmons. Nat. Ilist. N. York, vol. 5, pl. 35.

1862. Iarva, ehrysalis. Hirvis. Ins. Inj. Vegetat., Flint's ed., p. 300.

1862. Larva, ehrysalis. Morris, (quotes Bojs.-Lec.) S. nop. Lep. N. Amer., p. 54.

1869. Larva. Inaris. Entom. Corres., p. 280.

1869. Larva. Sautules, Canal. Eutoms, vol, 1, p. 76. 
1871. Iifo history, (col'd tigs.) W. II. Edlurarls. Butt. N. Amer., al serids. pl. (irapta, Nos. 4, 5.

1877. Laura, chrysalis. Pachemel. Inj. Ins. West, Itayolen liept., p. Tit.

1877. Larva. French. Trans. Jept. Agr. Ill., vol. 15, p. 152.

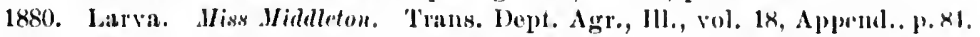

1880. Larva. Coguillett. 'Trans, Dept. Agr. 111., vol. 18, Appeal.,p. 16il.

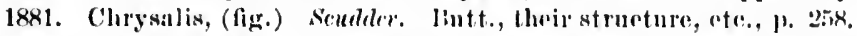

1881. Larva, chyrsalis. Pacliard, (iguotes Saumlers.) Ins. Inj. to Porest Trene, p. fis.

1882. Lifo history. Wr. IT. Edurards. Canad. Entom., vol. 11, p. 201.

188. Egge, larna, chrysalie. Firmuld. Butt, Maine, 1, 19.

1886. Lifo history, (condensed.) Frouch. Butt. Wast. U. States, p. 18t.

1886. Chrysalis, (fig.) Frewch. Butt. Bast. I. States, 1) 181.

Foon Plasts. Humulus, Ulmus, Crtica.

Grapta comma. Harris, (incl, vars.)

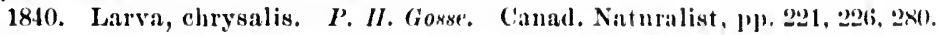

1811. Clirysalis. Harris. Its. Inj. Viggetat., 1st relit, p. 221.

1852. Larva, clirysalis. Iarris. Ins. Inj. Vegetat., Sol solit., p. 2o11.

1856. Larva. Fitch. 3d Rept. Ins. N. Y., p. 132.

1862. Larvi, ehrysalis. W. II. Edwards. Pros. Entom. Soc. Plilinl., vol, 1, p. 182.

1862. Chrysalis, (tig.) Harris. Ins. Inj. Vogetat., Flint's colit., p. 301, pl. I.

1862. Chrysalis. Morris. Synop. Lep. N. Amer., 1. s.t.

186.1. Larva, chrysalis. Linture. P'roc. Fntom. Soc. Philad., vol. 3, 1. ה. ה.

1871. Life history, (col'd figs.) W. II. Elwards. Butt. N. Amer., 2ul sirries., pl. Grapta, No. 2.

1871. Larva, chrysalis, (col'd ligrs.) W. H. Edu'urls, (var. dryas.) Low. cit., pl. Grapta, No. 3.

1877. Larva. French. 'Trans. Dept. Agr. Ill., vol. 15, p. 159.

1877. Larra. Packard. Inj. Ins. West, Itayden's Rept., p. 774.

1880. Laurva. Miss Midlleton. Trans. Dept. Agr. Ill., vol. 18, A plend., pr, $\times 1$.

1880. Linva. Coquillett. 'Trans. Dept. Agr. Hl., vol. 18, $\Lambda$ plend., p. 1fis.

1881. Iarva, chrysalis. Packurl, (rnotes W. H. Edwards.) Ins. Inj. Forest 'Trees, p. 66.

1881. Egg, (fig.) Scudler. Butt., thein structure, etc., p. 99.

1882. Lifo history. W. H. Eillwards. Cánad. Entom., vol. 11, p. 1 (19.

1884. Egg, larva, chrys. Fernald. Butt. Maine, p. 51.

1886. Life history. French. Bntt. East. U. States, I. 186.

FoOD PLANTs. IIumulus, Tlmus, Urtica.

Grapta satyrus. Edw.

18i2. Larva, chrysalis, (col'd fig.) II. H. Liduralls, (quotes Stretwh M. S.) Butt. N. Amer., 2d series, pl. (irapta, No. f.

1873. Larva. IIy. Edwards, (fuotes Stretel, MS.) I'roe. Cal. Acal. Sc., vol. 5, p. 168.

1873. Chrysalis. Hy. Edwarls. Loc. cit., 1. 168.

1875. Larva. C. W. P'earson. Canad, Entom., vol. 7, p. 216.

Foon Prant. Urtica.

Grapta rusticus. Edw.

1879. Larva, clrrysalis, (col'd fig.) W. H. Eidwards. Butt. N. Amer., 2d keries, il. Grapta, No. 3.

\section{Grapta faunus. Edw.}

1864. Larva, chrysalis, Lintner. Proc. Entom. Suc. Philad, vol. 3, 11. in.

1875. Larva. Caulficld. Canal. Entom., vol. 7, p. 49.

1880. Larva. Miss Middletou. Trans. Dept. Agrr. Ill., vol. 18, A jpend., p. Bi.

1881. Clurysalis, (fig.) Scuder. Butt., their structure, etc., p. 12.

1884. Egg, larva. Fomuld. Butt. Maine, p. 52. 
1884. Chrysalis, (figr.) Ferualit. Loc. cit., p. 52.

1886. Larva. Firnch. Butt. Wast. U. States, ]. 188.

1866. Chrysalis, (fig.) Hrewh. Lac. cit., p. $1 \times x$.

Foon l'Lavit. Salix.

- Grapta silvius. Edw.

1873. Larva, chrysalis. Hy. Eilwarls, (as G. zeplyyrus in error.) l'res., ('ill. Aeat. Se., vol. 5, p. 169.

Food Prant. Azalen occidentalis.

Grapta zephyrus. Edw.

1879. Larva, chrysalis, (col'd fig.) W. II. Edluarls. Inutt. N. Amer., 2d series, pl. Grapta, No. 3.

\section{Grapta progne. Cram.}

1810. Larva, chrys. P. H. Gosse. Canatl. Naturalist, P. 219, (as (i. e-alegenteum.)

1811. Larva, chryalis. Marris. Ins. Iuj. Vegotat., 1st edlit., p. 222.

18,5. Larva, chrysalis. Hurris. Ins. Inj. Vegetat, gal wit., p. 213.

1650. Larva, chrysalis. Fitch. 31 Rept. Ins. N. York, 1. 1330.

1862. Chrysalis. Marris. Ins. Inj. Vegetat., Flint's silit., p. ¿in1.

1869. Larva, (lorief.) Harris. Eutom. Corres., p. 280.

1871. Larva, chrysalis. Le Barou. 2d Illinois Rejt., p. 59.

1871. Egg. Scudder. Rept. Peabody Acal. Sc., 1. 34.

187l. Larva, (brief.) Samuders. Rept. Entom. Soc. Ontario, p. 376.

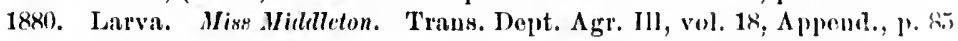

1880. Lifo history. W. II. Edwarls. Canad. Entom, vol. 12, ¡. 9.

1881. Larva. P'uckard, (quotes Fiteh.) Int. Inj. Forest, Trees, p. 6t;.

1883. Larva, clirysalis. Sannders. Ins. Inj. Fruits, p. 317.

188.1. Egge. larva, chrysalis. Fornald. Butt. Maine, p. 51.

1886. Lifo history. French. Bntt. East. U. States, p. 191.

Foon Plant. Ribex of various species.

Grapta j. album. Bois.-Lec.

1864. Larva, chrysalis. Lintucr. Proc. Nutom. Soc. Philad., vol. 3, p. 5,

1884. Lavia, chrysalis. Frumla. Butt. Maino, p. 5i.

1886. Larva, chrysalis, Frnch. Bntt. East. U. States, p. 193.

Vanessa antiopa. F.

1828. Larva, clurys., (briof.) Strphens. Ill. Brit. Entom., Haust., vol. 1, 1. 1.

1810. Larva, chrys. P. IT. (iosse. Canaul. Naturalist, p. 291.

1841. Larva, clirys. Murvis. Ins. Inj. Vegetat., 1st etit., p. 21!).

1812. Larva, (brief.) Duncan. Natural. Library, voi. fi, p. 169.

1852. Larva, chrys. Ilarris. Ins. Inj. Vegetat., 2t edit., p. 238.

1854. Larva, chrys., (col'd figs.) Emmons. Nat. Hist. N. York. vol. 5, p. 207, pl. 6.

1855. Larva, chrys.. (col'd figs.) Westuood. Brit. Butt., p. 50, pl. ד.

1856. Larva, chrys. Chrnu-Lucas. Encye. Nat. Hist. Papillons, vol. 1, p. 103.

1857. Larva, chrys., (hrief.) H. T. Stainton. Brit. Butt., Motlis, vol. 1, 1. 39..

1859. Larva, chrys. Ehwarl Newman. Butt. Young England, p. 4.

1862. Larva, chrys., (fig.) IIaris. Ins. Inj. Vegetat., Flint's chit., p. 297.

1861. Larvi, chrys. Jaeger. Life N. Amer. Ins., p. 202.

1864. Larva, chrysalis. Lintner. Proc. Entom. Soc. Plital., vol. 3, p. 59.

1869. Larva. Harris. Entom. Corres., p. 280.

1869. Larva. Saunders. Canal. Entom,, vol. 1, p. 75.

1871. Egg. Sculler. Rept. Peabody Aead. Sc., p. 40.

1878. Larva, chrysalis, (brief.) G. II. Perkins. 5th Rept. Vormont Boart Agr., p. 265.

1879. Larva, chrysalis, (fig.) Mrrs. I. P. Bullard. Insect Lives, P. 40.

1880. Larva. Miss Mirllleton. Trans. Dept. $\Lambda$ gr. Ill., vol. 18, Appentl, p. 8.'.

1880. Larva. Coquillett. 'Trans. Dept. $\Lambda$ gr. III., vol. 18, $\Lambda$ ppentl., p. 163. 


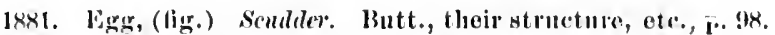

16x1. Latrva, elarysalis, (figr.) scudeler. Isoc, eit., p. 37.

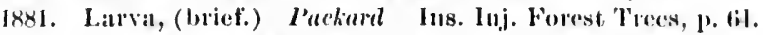

18xa. Larva, chrysalis. W. F. Kirby. Europ. Butt. and Motlis, p. 12.

1884. Ligg, (lig.) Feruald. lintt. Maine, 1. 56.

18x1. Latra, cloysalis. Fernuld. Loc. cit, p. if.

1885. Egg, lavia. Iy. Eduram. Kingsley's Stand. N. IIist., vol. 2, p. 187.

1886. Fggr, (fig.) French. Butt. Last. U. States, p. 191.

18x6. Larva, chrysalis. Finch. Loc. cit., 1. 194.

Foon Plast. Sulix.

Vanessa californica. Bois.

1863. Larva, (lurief) Behr. Proc. Cal. Aeal. Sc., vol. o, p. 123.

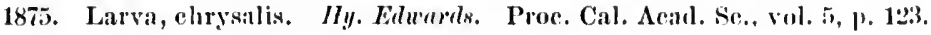

Foon Plast. Cemothus thyrsiflorus.

Vanessa milbertii. (iolt.

1810. Larva. ¿. H. Gosse. Camad. Natmialist, pl. 107, 2201.

1802. Larva, elursalis. IIaris, Ins. Inj. Vegetat., 2d relit., j. 303.

1863. Larva. Saunders. P'roc. Entous. Soc. Plibad., vol. 2, 1. 28.

1864. Larva, claysalis. Linture. Proc. Entom, Soc. Philad., vol. 3, p. 61.

1869. Lapva. Somnders. Camad. Entom., vol. 1, p. Tf.

1873. Iarva, clırysalis. IIy. Eduarls. Proc, Cal. Aead. Se., vol. 5, p. 170.

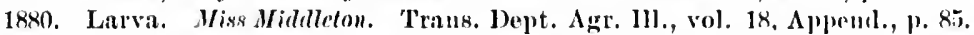

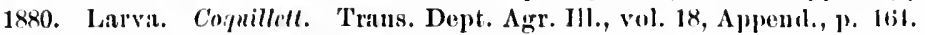

1881. Eugg, (figr.) Sculder. Butt., their structuro, etc.; p. !y9.

1881. Larva. Fernald. Butt. Maine, p. 57.

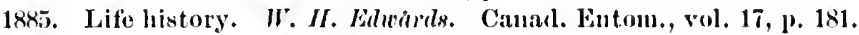

1880. Iarva, clırysalis. French. Br:tt. Fast. U. States, 1. 195.

Food Prant, Urtica.

Pyrameis atalanta. L.

1828. Larva, chrysalis, (hrief.) Stephens. Ill. 13yit. Entom., Hanst., vol. 1, 1.46.

1812. Larva, (brief.) Duncan. Natural. Library, Entom., vol. 6, p. 171.

1855. Larva, chrysalis, (col'l fig.) Westwood. Brit. Butt., j). 53, pl. 8.

1856. Larva, clurysalis, Ch'mu-Lueas. Encyc. Nat. II ist. Papillows, vol. 1, 1, 103.

1857. Larva. Stainton. Man. Brit. I3ntt., vol. 1, p. 38.

1859. Lav'va, chrysalis. Eilw. Néman. Butt. Young Eugland, p. 10.

1862. Lav'a, clurysalis, (fig.) Ilarris. Ins. Inj. Vegetat, Flint's edit., p. 291.

1862. Larva, clirysalis. Morris. Synop. Lep. N. Amer., p. 54.

1861. Larva. Jaryer. Lifo N. Amer. Ins., p. 203.

1869. Larva. Harvis. Entom. Corres., p. 279.

1873. Larva. Hy. Eihwards. Proc. Cal. Acarl. Sc, vol. 5, p. 169.

1878. Lavva. Birch. Entom. Montlı. Mag., vol. 13, ]. 209.

1880. Larva. Miss Middleton. 'Trans. Dept. $\Lambda$ gre. Ill., vol. $18, \Lambda$ plend., p. 86.

1881. Eggr, (lig.) Sendeler. Butt., their structure, otc., p. 8.

1881. Nest, (fig.) Sculder. Loc. cit., p. 109.

18x2. Larva, chrysalis. H. F. Firby. Enrop. Butt. and Motlis, p. 11.

1882. Lifo listory. W. II. Edwards. Canal. Eutom., vol. 11, 1. 22!.

1883. Lifu history, (cont'l.) W. H. Edwarls. Loc. eit., ve1. 15, p. 14.

188. Egrg, (fig.) Fonald. Butt. Maine, p. 58.

1884. Larva. Fruall. Loc. cit., p. 58.

1886. Life history, (condensed.) French. Butt. Fist. U. States, p. 197.

1886. Egg, (fig.) French. Loc, eit.

Food Plants. Humulus, Bahmevia, Ortica.

Pyrameis huntera. F.

1797. Larva. Abbot-Smith. Isep. Ins. Goorgia.

1833. Larva, chrysalis, (col'd fig.) Bois.-Lec. Ilist. Lep. N. Amor., p. 180, pl. 48. 
18tie. Larva, chrysalis. Morris, (quoles Bois. and Loe.) Synop. Lep. N. Amer., 1. (60).

1stie. Larva, ehrysalis, Marris, Ins. 1uj. Vegetat., Flint's edit., p. 293.

18ti. Larva, chrysalis. Lintuer. Proc. Entom, Soe. I'lithul, vol. 3, p. ti3.

1x6is. Latra. Sumuders. Canad. Entom., vol, 1, 1. 105.

1873. Larva. My. Eimards. Proc. Cirl, Aead. Se, vol, 5, p. 170.

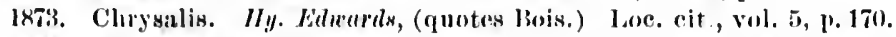

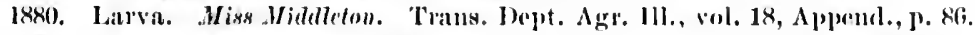

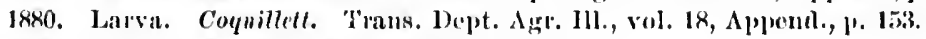

1881. Latra. Frrmall. Butt. Maine, p. E8.

1886. Larva, chrysalis. French. Butt. East. V. States, p. 198.

Foo1, I's.axts. Gnaphalinm, Antennaria.

Pyrameis cardui. L.

1828. Larva, chrysalis, (brief.) Stephens. Illnst. Brit. Eutom., Hanst., vol. 1, p. 47.

1812. Larva, chrysalis, (brief.) Luncou. Natural. Library, Entom., vol. 6, p. 17 i.

18.4. Larva, chrysalis, (col'd figs.) Limmons. Nat. Hist. N. York., vol. n, pl.46.

185is. Larva, chrysalis, (col'd ligs.) Hestroot. lirit. lintt., p. 5i, pl. 8.

18506. Lanva, chrysalis. Chemu-Lncus. Encye. Nat. Ilist. Papillons, vol. 1, p. 104.

1857. Lavia. Stainton. Man, Brit. Butt., p. 37.

1859. Larva, chrysalis. Eilw. Newmun. Butt. Young England, p. 10.

1802. Larva, chrysalis. IIarris. Ins. Inj. Vegetat., Flint's edit., p. 292.

1869. Larva, chrysalis. Morris. Syuop. Lep. N. Amer., p. 60.

1864. Larva. Jasger. Life N. Amer. Insects, p. 20-1.

1869. Larva. Senuders. Canad. Entom., vol. 1, p. 93.

1869. Larvin, chrysalis, (tigs.) Harris. Entom. Corres., p. 277, pl. 1.

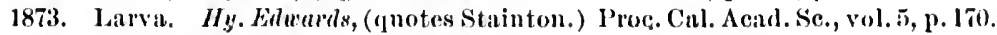

1876. Larva. Sculder. Amer. Naturalist, vol. 10, P1!. 392, 602.

1880. Larva. Miss Middlcton. Trins. Dept. Agr. Ill., vol. 18, Append., p.87.

1880. Larva. Coquillett. Trans. Dept. Agr. Ill., vol. 18, Append., p. 153.

1881. Larva, (briof.) Gumlluch. Eutom. Culani. p. 40.

1882. Larva, chrys. W. F. Kibly. Europ. Butt. and Motlis, p. 13.

1884. Larva. Ferwald. Butt. Maine, p. 59.

1886. Lava. French. Butt. East. U. States, p. 200.

Food Puants. Cuicus, Curduns, Althrat, Urtica.

Pyrameis caryæ. Jülbn.

1863. Larva, (brief.) Behr. Proc. Cal. Acad. Sc., vol. 3, p. 125.

1874. Larval stages, chrysalis. Iyy. Lilurrols. Loc. cit., vol. 5, p. 329.

Food Plant. Lavalera assuryentifloru.

Junonia conia. Jlïln.

1797. Larva, (col'd fig.) Ablott-Smith. Lep. Ins. Georgia, pl. 8.

1333. Larva, chrysalis, (col'l fig.) I'vis-Lee. Hist. Lep. N. Amer., p. 182, pl. 4!.

1862. Larva, chrysalis. IIarris. Ins. Inj. Vegotat., Flint's edit., p' 293.

1873. Larva. IIy. Ellwarls, Proc. Cal. Aeall. Sc, vol. 5, p. 171.

1873. Chrysalis, (loriof.) Hy. Eduarils. Loc, cit., vol. 5, p. 171.

1880. Larva, (briof.) Sanmie's. liept. Entom. Soc. Ontario, p. 40.

1880. Larva. Miss Midelleton. 'I'rans. Dept. $\Lambda$ gr. III, vol. 18, $\Lambda$ ppend., p. 87.

188.. Larva. Fernald. Butt. Maine, p. 60.

1886. Larva. French. Butt. Bast. U. States, p. 201.

Food Peasts. Plentugn, Gravelin, Antirrhinum.

Limenitis ursula. Fal.

1833. Larva, chrysalis, (col'd figs.) Rois.-Lee. IIist. Lep. N. Amer., p.199, pl. 53.

1859. Larva, (lirief.) I. II. Gosse. Lettors from Alabama, p. 123.

1862. Iarva, chrysalis. Morrix, (g̨notes Bois.-Leo.) Synop). Lop. N. Am., p. 6í.

1862. Larva, ehrysilis. Haris. Ins. Inj. Vegetat., Flint's edit., p. 283. 


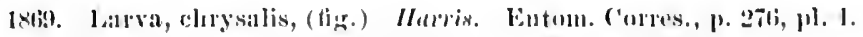

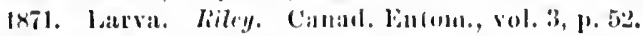

1877. Larva. Freftele. Trans. Dept. Agr. 11l., vol. 15, f1. 151.

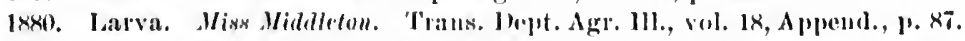

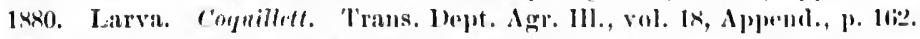

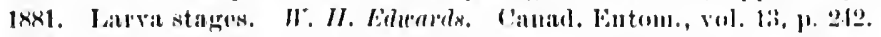

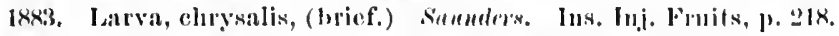

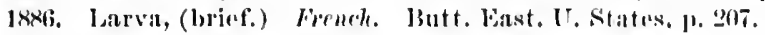

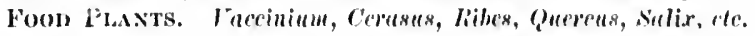

Limenitis arthemis. Drury, (inel. vars.)

18.10. Larva, cluys. (ligs.) P. H. Gosee. Canad. Naturalist, ll. 224i,221.

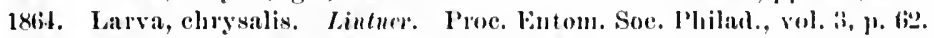

1875. Larva. T. L. Merel. Canad. Entom., vol. T, p. 16i3, (var. proserpina.)

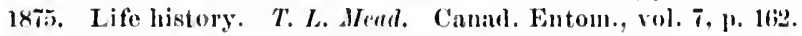

187i. Larva. Srudder. Psyclie, vol, 1, 1. 11.

1879. Life history. W. H. Etwords. Canad. kintom., vol. 11, p. 221.

187!. Lifo history. IT. M. Ehwerds. Canad. linton., vol. 11, p. ezt, (var. prosorpina.)

1879. Life history, (col'd figs.) W. H. Eillowds. Hutt. N. Auner., gl series, pl. Limenitis, No. 1.

1sist. Nest, (tig.) Sculder. Butt., their structure, etr., p. 113.

1s81. Vigg, lava, ehrysalis. Fevelll. Butt. Maine, 1) liz.

18x\%. Lifo history, (condensed.) French. Butt. East, U. States, p. 208.

Foan: Prants. Cintityou, Stlix.

Limenitis disippus. riolt.

1833. Larva, ehrysalis, (col'll figs.) Bois.-L'r. Uist. Lep. N. Amer., p. 20.4, 1). 55.

1856. Larva. Fitch. 3d Rept. Ins. N. York, p. 37!?.

18ti2. Larva, chrysalis. Morris, ('pnotes Bois.-Lec.) Synop. Lep. N. Aner., p. 66.

1862. Lalval, chrysalis. Harris. Ius. Inj. Vegretat., Flint's eolit., 1. $2 \times 2$.

1864. Larva, chrysalis. Lintuer. Proc. Eutom. Soc. Philad., vol. 3, p. (ii3.

1869. Larva. Saunders. Canad. Entom., vol. 1, p. 94.

1869. Larva, elnrysalis. Amer. Eutom., vol, 1, p. 193.

1871. Larva. Riley. Canal. lintom, vol, 3, p. 52.

1871. Erg, (figs.) likly. 3d Missouri Rept., p. 153.

1871. Larva, chrysalis, (figs.) Riley. Loc, cit., plr. 1:53-157.

187t. Life history, (tigs.) Sanuders. Canal. Enfont., vol. f, p. Af,

187t. Larva, chrysalis, IIy. Edtrads. I'roe. Cal. Aead. Se., vol. 5, p. 330.

1877. Larva. French. 'Trans. 1hept. Agr. Ill., vol. 15, 1. 15l.

1879. Life history. W. II. Edwards. Canad. Entom., vol. 11, 1. 221.

1881. Nost, (fig.) Scudder, (quotes Riley.) Butt., their struetnre, etc., p. 112.

1881. Eugr, (fig.) Scudder, (quoten Riley.) Loc. cit., p. 10.

1881. Larva, chrysalis, (figs.) Sculler, (quotes Riley.) Butt., their structum, otc., pp. $22,45$.

1883. Egro, larva, clirysalis, (ligs.) Saunders. Ins. Inj. Fruits, ple. 169, 170.

1881. Ligg, larva, chrysalis, (figs.) Fernald. Bntt. Maine, ple. 6i3, 61.

18*6. Egg, larva, chrysalis, (figs.) Fonch. Butt. East. U. States, p. 211.

188!. Lifo history, (condersed.) French. Loc. cit., p. 211.

Food Praxts. Salix, Populus, ete.

Limenitis eros. Edw.

53.

1880. Larval stages, chrysalis. W. $M$. Educards. Camal. Eutom., vol. 12, p. 2.16.

1883. Life history, (col'd ligs.) W. II. Wilwards. Butt. N. Aner., 2l series, pl. Limenitis, No. 2.

1886. Lifo listory, (comlensed.) Frneh. IButt. Fist. U. States, p. 214. 
Limenitis lorquini. Biris.

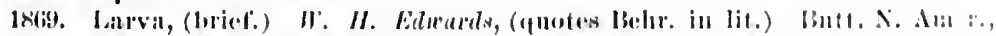
pl. Limenitis, No, 3.

1873. Chrysalis. Hy. Eillurals, Proe, Cal, Acad, Se., vol, 5, p. 171.

187.1. Larva. Hy. Edurards, Loce eit., vol, 5, p. 330.

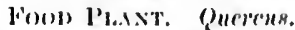

Heterochroa californica. Intler.

1873. Clirysalin. Hy. Eitreurls. Proe. Cal, Aeal. Se., vol. 5, p. 171.

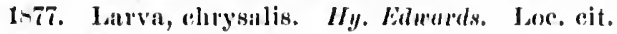

Foon l'tast, gue'ens.

Apatura clyton. Bois.-Lee., (includ. vass.)

1833. Istrva, chrysalis, (col'd tigs.) Bair.-Lec. IJist. Lep. N. Amer., p. 208, pl. in.

18\%6. Larva. Fitch. Bal hept. Ins. N. York, p. 380.

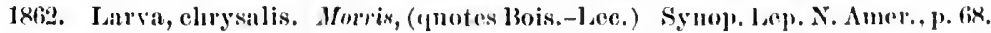

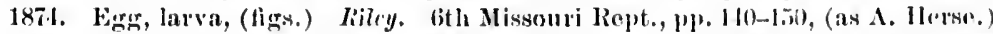

1876. Lifo history, (col'l ligs.) W. II. Edurards. Butt. N. Amer., 2 l series, pl. Apatura, No. 2.

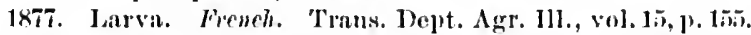

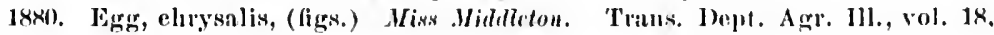
Append., p. 8:).

18x!. Egg, larva, chrys., (iigs.) Scudder, (after libley.) Butt., their structure, etc., p. 262.

1881. Larva, ehrys. Gunduch. Eutom. Cubama, p. 133, (as Doxoeopa Idyja. 111,11.)

1886. Egg, litria, elirys., (figs.) French, (after Riley.) Butt. Wast. U. States. 1. 219.

1886. Lifo history, (eomlensed.) Freuch. Loe. cit., p. 219.

Fuon I'IAxT. Celtis occidentalis.

Apatura flora. Edw.

1881. Lilo history. W: H. Edwards. Canal. Nutom., vol, 13, p. 81.

1886. Lifo history, (condensel.) French, (quotes W. II. Jilwards.) Butt. East. U. States, p. 222.

Apatura celtis. Bois.-Lec.

1833. Larra, clurysalis, (col'd figs.) Boir.-Lec. Hist. Lep. N. Amer., p. 210, wl.57.

1862. Larva, chrysalis, Morris, (quotes Bois.-Lce.) Synop. Lep. N. Amer., p. lia.

187.1. Egrg, (figr) Riley, (as A. lycaon.) Bth Missouri Rept., ply. 137-1501).

187.1. Larva, elurysalis, (figs.) litley. Loe. cit., pp. 137-150.

187is. Life history, (col'd figs.) Ir. M. Edworls. Butt. N. Amer., 2d series, pl. Apatura, No. 1.

1877. Larva. Frech. Trans. Dept. Agr. 11l., vol. 15, p, 15\%.

1880. Larva. Miss Middlitou. Trams. Dept. $\Lambda \mathrm{gr}$. Ill., vol. $18, \Lambda$ ppend. p., 88.

1881. Ligg, larva, (figs.) Seutle's. Butt., their strueture, ote., p. 26 is.

1880. Lifis history, (figs.) Frnch. Bntt. East. U. States, p. 216.

Foon P'Lant. Cellis occidentulis.

Apatura alicia. Edw.

1881. Latra, (briof.) H. H. Efluards. Amer. lintom., vol, 1, 2d series, p. 206.

1880. Lifo history. W. M. Eilwards. l'syche, vol. 3, pl. 123-127.

Paphia troglodyta. F'ill.

1870. Larva, chrysilis, (figs.) Riley. 21 Missonri Report, pp. 125-128.

1871. Larva, elırysalis, (col'd figs.) H. H. Edwards, (!notes llaylurst in lit.) Butts. N. Am., 2l series, pl. Puphia, No. 1.

1873. Lifo history, (figs.) Riley. 5th Missouri Rept., pp. 145-119.

1880. Jarva, chrysalis, (figs.) Miss Mitdletou. 'Truns. Dept. Agr. Ill., vol. 18, $\Lambda_{\text {] }}$ peud., p. 89.

1881. Larva, nest, chrysalis, (figs.; Scudder, (after Riley.) Butt, their structure, etc., p. 111.

1886. Larva, elırysalik, (figs.) Freweh. Butt. East. U. States, p. 227. 


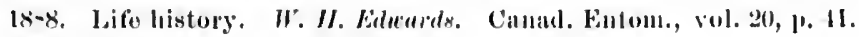

Fu(i) l'taxis. Croton crupitatum.

Debis portlandia. Fab.

1825. Larvi. Soy. N. Amer. Entenolongy, vol. 2.

1833. Larva, elurysalis, (col'el tig.) Boiselued-Leconte. Ilist. Lep. N. Amer., p. 296, pl. Ex.

1862. Iarva, chrysalis, (brief, Marrix, (fuotes say.) Symop. Lep. N. Amer.. p. 7!), (as D. and comatehin.)

1862. Larva, chrysalis. Morvix, (4uotes Bois.-Lee.) Loe. eit., p. 79.

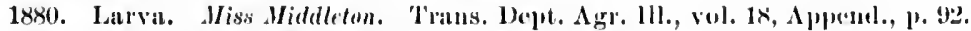

1882. Lifo history. H. H. Edmorld. Canal. Entom., vol.11, p.81.

1881. Ligg, larva, dirysalis, Fernald. Butt. Maine, p. Tl.

1886. Lifo listory, (eondensed.) French. Butt. bast. U. States, 1. 2:i0.

Neonympha canthus. Bois.-Lee.

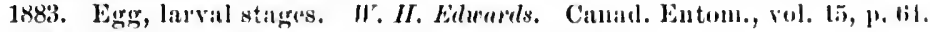

1884. Figg, larva. Fimald. Butt. Maine, 1. fix.

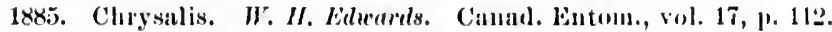

1886. Lifo histury, (brief.) French. Butt. East. U. States, 1. 232.

Neonympha gemma. Hlïlıl.

1833. Larvia, chrysalis, (col'd figs.) Boisdural-Laconte. Hist. Lep. N. Amer., 11. 62 .

1879. Lite history, (figs.) H. H. Ehreards. Cimat. Entom., vol. 11, p. 31.

1880. Larvi, ehrysalis. Miss Middleton. Trans. Dept. Agr. Ill., vol. 18, Append., 1. 91.

1881. Life history, (conlensed.) French. Butt. East. U. State's, p. 235.

Neonympha areolatus. Abb.-Sin.

1797. Larva. Ablot-s'mith. Lep. Ins. Georgia, rol. 1, hl. 13.

1833. Larva, clerysalis, (col't figs.) Boiselural-Leconte. IIist. Le[1. N. Amer., pl. 63.

1882. Life history. W. II. Ede'ards. Canad. Entom., vol.11, 1.163.

1886. Lifo history, (condensed.) French. Butt. Eist. U. Stattes, 1. 237.

Neollympha eurytris. Fal.

1833. Larva, chrysalis, (col'il figs.) Boisthum-Laconte. Hist. Lep. N. Amer., 1). 61.

1870. Egg, larval stages. Saumlers. Canad. Entous, vol. 2, p. 139.

1877. Larva. French. 'Trans. Dept. Agr. Ill., vol. 15, 1. 15ti.

1878. Life history. W. H. Edwards. Canad. Entom, vol. 10, 1. 195.

1880. Lgge, larva, chrysalis. Miss Midlleton. 'Trans. Dept. Arrr. IIl., vol. 18, Alpend., 1. 90.

1880. Larva. Coquillett. 'Trans. Dept. Agrr. Ill., vol. 18, Appenel., 1'. 181.

1884. Larval stages, chrysalis. Fermald. Butt. Maine, p. 67.

1886. Lifo history, (condensed.) French. Butt. Ealst. U. States, 1. 238.

Neonympha sosybius. Fal.

1833. Larva, chrysidis, (col'd figs.) Boistural-Leconte. Ilist. Lepr. N. Aner., pl. 63.

1877. Life history. W. II. Eilwards. Canal. Eutom., vol. 9, 1. 229.

1880. Lgg, larva, chrysalis. Miss Middleton. 'Trims. Dept. Agr. Ill., vol. 18, Append., p. 91.

1886. Life history, (condenserl.) Fench. Butt. East. U. States, p. 211.

Conomympha ampelos. Edw.

1887. Life history. W. IT. Edwarls. Canad. Entom., vol. 19, p. 42.

Satyrus alope. Fab., (includ. vars.)

1833. Larva, chrysalis. Boisdurul-Leconte. IIist. Lep. N. Aner., p. 228, pl. 59.

1854. Larva, chrysalis, (col'd figs.) Emmons. N. Hist. N. York, Eintom., vol. 5, pl. 33. 


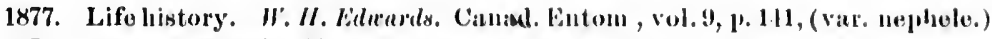

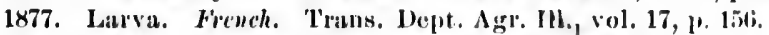

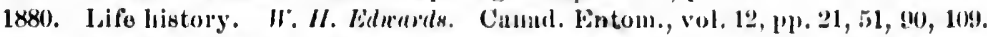

1880. Larva, ehrysalis. Miss Middleton. Trans. Mept. Agr. III., vol. 18, Append., 1. 42.

1880. Eggr, larva. Miss Midtllton. Loc. eit., p. 92, (var, nephole.)

1880. Larva. Coquillett. Loc. eit., 1) 180, (var. nephele.)

1881. Young larva, chrysalis, (tigs.) Scudder. Butt., their structure, etc., pl. $29-13$.

1882. Life history, (col'd tig.) I'. II. Lidwards. Butt. N. Amer., 2d series, pl. Satyrus, 2-3, (incl. vars, nephole-olympus.)

1884. Egg, larva, chrysalis, (tigss.) Firnald. 13utt. Maine, p. Tl.

1886. Chrysalis, (tig.) French. Bintt. East. 1). States, p. 266.

1886. Lifo history, (condensed.) Fiendh. Loce cit., 1. 296.

Satyrus charon. Edw.

1875. Egg. T. L. Wend. Rept. Lepid, Wheeler Exped., vel. 5, p.773.

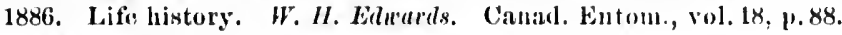

Chionobas iduna. Edw.

1879. Eggr. W. H. Ederards. Canaul, Entom., vol. 11, 1.113.

1879. Eggr, (col'd tigr.) I'.U. Eiluterls. Butt. N. Aner., 2ll sories, pl. Chionobas, No. 3.

Chionobas chryxus. West.-IIew.

1875. Egg. T. L. Mead. Rept. Lepid. Wheeles Exped., vol.5, 1. 777.

Chionobas ivallda. Mead.

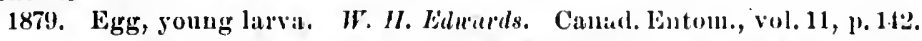

1879. Egg, young larva, (col'd figs.) W.II. Edherels. Butt. N. Amer., 2d series, 11. Chionobas, No.3.

Chionobas semidea. Sily.

1863. Egg, larva, chrysalis. Scuder. Charact. Insect Fauna, White Mountains, pp. 623, 625.

1874. Larva, ehrysalis, (figs.) Soudler. Geology N. Hampshire, pl. A.

Chionobas jutta. Hübn.

Egg, larva, (tìg'd.) Holmgren. Entom. 'Tidsch., vol. 7, p.151.

1888. Lifo listory. T. W. Fyle's. Canad. Entom., vol. 20, p. 131.

1889. Larva, notes on. T. IT. Fyles. Canal. Butum., vol. 21, p. 12.

Chionobas macounii. Edw.

1888. Egg, larva. I. Fletcher. A 'Trip to Nepigon, p. 12.

NotE.-It is believed that the larva of tho Satyrine feed exclusively on varions species of Grasses and Carices.

Libythea bachmanni. Kirtl.

1833. Larva, chrysalis, (col'd fig.s.) Boistluval-Leconte. Hist. Lep. N. Amer., pl. 64, (as L. motya.)

1874. Lifo history, (col'd figs.) F. II. Edluarly. Butt. N. Amer., 2d series, pl. Libythea, No.1.

1881. Life history. H. H. Edlicurls. Canad. Entom., vol. 13, p.226.

1886. Lifo history, (brief.) Hronch. Butt. Eilst. U. States, p.251.

Food Plant. Celtis.

\section{Eumenia atala.}

1875. Larval stages, clirgsalis, (figs.) Soudder. Meur. Boston Soc. N. History, vol.2, p. 113, pl. 14.

1888. Life history, (figs.) E. A. Schwartz. Insect Lile. U. S. Dept. Agr., vol. 1, p. 37.

Foon Plant. Zamia integrifolia.

Lemonias nais. Edw.

1883. Life history, (col'd figs.) W. II. Edwards. Butt. N. Amer., 2d series, pl. 11, pl. Lemonias, No. 1. 
Feniseca tarquinius. Fials.

1833. Larva, chrysulis, (figs.) lioialural-Levule. Ilist. Lep. N. Auer., p. 128, pl. 37.

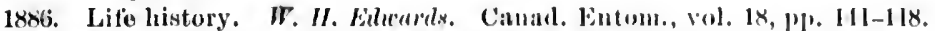

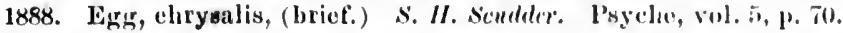

Feeds on $\Lambda$ phides. So lat, tho only carniverons hepidopterens larva known in our fallua.

Thecla hafesus. Cr.

1883. Larva, chrysalis, (col'd tigs.) Boistueral-Lecontc. Ilist. Lx'l. N. Aner., 1. 83, pl. 25.

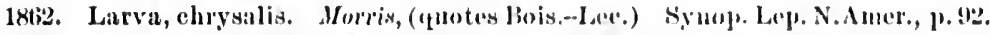

1886. Larva, chrysilis, (brief.) French, (tuotes dismis.) liutt. East. V.states, p. 256.

1888. Chrysalis. Hy. Edwards. Entom. Amer, vol. 3, p. 162.

Foon Plant. (Murens.

Thecla m. album. Bois.-Lee.

1833. Larva, chrysalis, (col'al tigs.) Boischuchl-Leconte. Ilist. Lep. N. Amer., p. 88, pl. 27. (as 'T. pisyche.)

1833. Larva, chrysinlis, (col'd tigs.) Boisdued-Leconte. llist. Lep. N. Amer., p. 86, pl. 26.

186i2. Larva, chrysalis. Morrix, (guotes liois.-1.ee.) Synop. Lep. N. Amer., p, 93.

1880. Larva, chrysalis. Miss Midlllcton. Trans. Dept. Agr. Mll, vol. 18, $\Lambda_{1-}$ pend., p. 92.

1886. Larva, elnysalis, (Inief.) Freneh. Butt. East. U. States, 1. 2056.

Foob Piants. (Gercus, Astragalus.

Thecla favonius. Abb,-Sm.

1797. Larva, chrysalis, (col'd tigrs.) Abbot-simith. Lef̧. Ins. (icorryia, rol. 1, pl. 14.

1886. Larva, chrysalis, (brief.) French, (1uotes $\Lambda$ thb.-Sim.) Isutt. Last. U. States, 1. 258.

Food Plast. Qucreus.

Thecla humuli. Harr.

1833. Larva, chrysalis, (col'd figs.) Boistlurul-Lecoute. Hist. Lep. N. Amer., p. 90, pl. 28, (as T. hyperici.)

1833. Larva, chrysalis, (col'd ligs.) Boistural-Lecoute. Ilist, Lep. N. Amer., p. 99, pl. 31, (as 'T. liparopls.)

1852. Larva, (brief.) Harris. Ins. Inj. Veget., 2d edit., p. 235.

1862. Larva, chryealik. Morris, (guotes Bois.-Lec.) Synop. Lep. N. Amer., pp. 96,97 , (as T. liparops.)

1862. Larva, chrysalis. Morris, ('notes Bois.-Lec.) Synop. Lep. N. Amer., p.94, (as T. hyperici.)

1862. Larva, chrysalis. IJarris. Ins. Inj. Veget., Flint's edit., f. 27i6.

1877. Larva. I'ackurd, („uotes Harris.) Inj. Ins. West, Ilayden's Rept., l. 773.

1880. Larva. Miss Middleton. 'Trans. Dejit. Agr. Ill., vol. 18, Append., p. 92.

Food Plant. Humulus.

Thecla acadica. Elw.

1868. Larva. Saunders. Camal. Entom., vol. 1, p. 95.

1869. Larva, (col'l fig.) W. II. Eilururls. Butt. N. Amer., J.st series, pl. Thecla, No. 1.

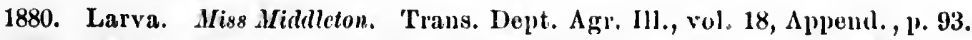

1886. Larva. French, (quotes Saunder's.) Butt. Wast. U. States, p. 261.

Foon Plant. Salix.

Thecla calanus. IIübn.

1833. Larva, chrysalis, (col'd figrs.) Boisdural-Leronte. Hist. Lep. N. Amer., p. 92, pl. 29, (as 'I'. falacer.) 


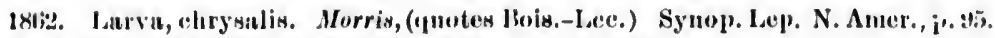

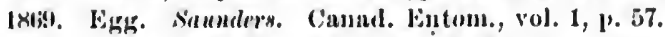

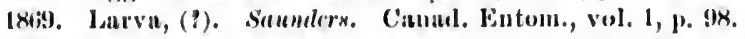

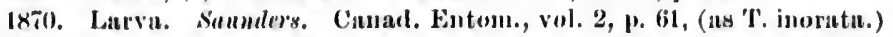

1880. Larva, (1) Miss Millleton, (quotes Sannlers.) 'Truns. Dept. Agr. III., vol. $18, A_{\text {ppend., p. } 93 .}$

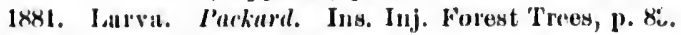

1886. Larva, chrysalis. French, (quotes Samnclors.)

Butt. Eitst. U. States, P. 264.

Foot) Puant. Quercun.

Thecla strigosa. Harris.

1868. Larvil. Suluder's. Canaul. Entonu., vol. 1, p. 99.

1869. Larva, ehrysilis. W. II. Lidirards, (quotes Sinuder's.) IButt. N. Amer., lot series, pl. 'Thecha, No. 2.

1880. Larva. Miss Jidllleton. 'Trans. Dept. Agr. 111., vol. 18, Append., p. 92.

1683. Larva, chrysalis. Saunders. Ins. Inj. Fruits, 1. 176.

1×8.1. Larva. Firmald, (quotes Samulers.) Butt. Maine, p. T8.

1886. Larva, chrysalis. French, (quotes Sinunders.) Butt. East. U. States, 1. 267.

Foon Plant. Querchs, ete.

Thecla smilacis. Bois.-Lec.

1883. Larva, chrysalis, (col'd figs.) Boisdnt'd-Le'ente. IIist. Lep. N. Anter., l. 107, pl. 33 .

1862. Larva, ehrysalis. Morris, (quotes Boin.-Lec.) Synop. Lep. N. Amer., 1. 99.

1880. Larva, chrysalis. Miss Midlleton. Trans. Hept. Agr. Ill., vol. 18, Appeud., p. 93.

188. Larva, chrysalis, (brief.) Saunders. Canad. Enton., vol. 16, p. 52.

1886. Larva, chrysalis, (briof.) French. Butt. East. U. States, 1. 268.

Food Plant. Smilax rotumlifolia.

Thecla poeas. Hübu.

1880. Larva; (brief.) Kiley. Amer. Entom., vol. 1, 2d sories, p. 201.

1880. Larva, (brief.) Miss Middleton. Trans. Dept. Agr. Ill., vol. 18, Append., p. 93.

Food Plant. Gossypinm.

Thecla irioides. Bois.

1878. Larva, chrysalis. Hy. Edwartly. Proc. Cal. Acad. Se., Juno.

Food Plant. Sctum spathulifolium.

Thecla irus. (iodt., (incl. vars.)

1833. Larva, ehrysalis, (col'd figs.) Boisdural-Lecoute. Hist. Lep. N. Amer.. p. 101, pl. 31.

1833. Larva, chrysalis, (col'd figs.) Boisturul-Leconte. Hist. Lep. N. A mur., p. 103, pl. 32, (as 'T. arsace.)

1862. Larva, chrysalis. Morris, (quotes Bois.-Lee.) Synop. Lep. N. Amer., p. 97, (as 'T. arsace.)

1862. Larva, ehrysalis. Morris, (quotes Bois.-Lec.) Synep. Lep. N. Aner., p. 97, (as 'T. iris.)

1880. Larva, chrysalis. Miss Midtllton. Trans. Dept. Agr. Ill., vol. 18, $\Lambda_{\text {p- }}$ pend., p. 94.

Food Prant. Tuccinium corymbosum.

Thecla henrici. Grote-Rob.

1881. Life history. W, H. Edwards. Papilio, vol. 1, p. 150.

1884. Lifo history, (condensed.) Fel'wald, (quotes W. H. Edwards.) Butt. Maine, p. 81.

1886. Life history, (conlensed.) French, Butt. East. U. Stutes, p. 274.

Food Plant. Prunus. 
Thecla niphon. Hiilı.

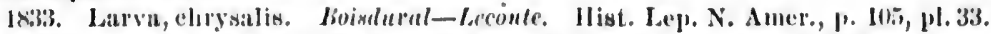

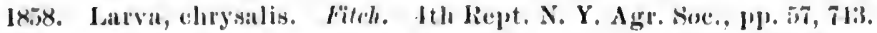

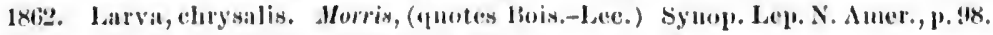

18tix. Larva. Sanmlers. Camal. Fintom., vol, 1, J. !ni.

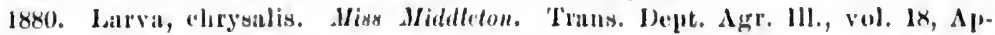
Jenl., P. 94.

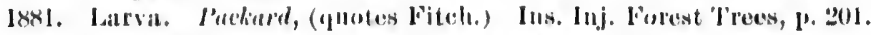

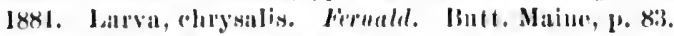

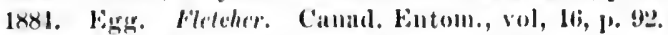

188ti. Larva, chrysalis, (hrint.) Frenth. Butt. Fast. U. States, p. 2iti.

Foon Plaxt. l'inur.

Thecla titus. Fials.

1833. Larva, chrysalis. Borixdural-Leconte. Ilist. Lep. N. Amer., p. 109, pl. 31. 1863. Lallva, clupsalis. Morrix, (quotes Bois.-Jae.) Symop. Jap. N. Amer., p. 102.

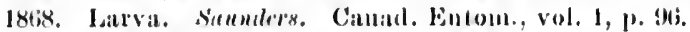

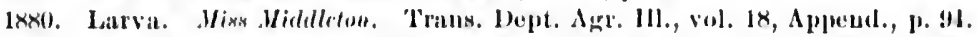

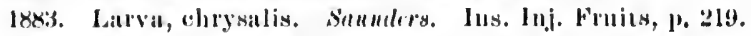

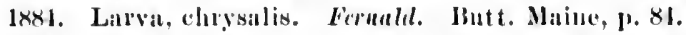

1886. Larva, cluysalis, (brief.) French. Butt. East. U. States, 1. 2ī8.

Foon P'I.ANT. (iucreus.

Chrysophanus thoe. Bois.-lee.

18ti8. Eigg. Setunders. Canal, Eutom., vol. I, p. 57.

1880. Eggr. Miss Midlleton, (qunotes Samuders.) 'Trans. Dept. Agrr. III., vol. 18, Append., p. 95.

1881. Eggr, (tig.) Scudder. Butt., their structuro, etc., p. $\times$.

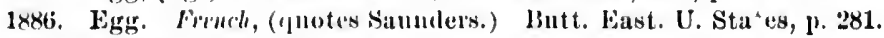

Foon Plaxt. I"uly!joutum.

Chrysophanus xanthoides. Bois.

1887. Eugr. Hy. Elhurdl. Eutom. Amer., vol. 3, 1. 162.

Chrysophanus helloides. Hois.

1875. Larva, ehrysalis. T. L. Meat. Rept. Lep. Whecler Expd., vol.5, p. 780 .

plond.,

Clirysophanus epixanihe. Bois.-lee.

1868. Eggr. Sauneiers. Canad. Entom., vol. 1, p. 57.

1886. Eigg. French, (quotes Saunders.) Rutt. East. U. States, p. 282.

Clırysophanus hypophleas. Bois.

1810. Eggr. P. II. Gosse. Canad. Naturalist, p. 221.

186\%. Larva, (brief.) Packard. 2a Rept. Nat. Llist. Geol. Maine, p. 166.

1863. Larva, eluysalis. Iherris. Ius. Inj. Vegretat., Flint's tolit., 1. 27..

18i8. Larva. Stunders. Camad. Eutosn, vol. 1, p. 4.

1880. Larva. Miss Midelleton. 'Trans. Dept. Agr. 1ll., vol. 18, Apjend., p. 95.

1881. Egrg, larva, chrysalis, (tirs.) Sculder. Butt., their structure, ete., pp. 7, $20,31,43$.

1884. Larva, chrysalis, (ligs.) Feruald. Butt. Maine. p. 88.

1885. Larva. IIy. Elleurds. Kingsley's Stand. Nat. Ilist., vol. 2, p. 476.

1886 Eggr, larva, ehrysalis, (ligrs.) French, (quotes Saunder's and Seudder.) Butt. East. U. Stites . 283.

Foon Plast. Liumcx acetoselia.

Lycæna lycea. Edw.

1875. Larva, (brief.) T. L. Mead. Rept. Lep. Wheeler Expd., vol, 5, p. 785.

Lycæna pembina. Edw.

1868. Larva. Sauders. Canad. Eutom., vol. 1, p. 12.

Butt. Lycæna melissa. Fdw.

188.!. Life history. W. IT. Edwards. Papilio, vol. 4, p. 91.

Foon Plant. Astragalus.

BuLL. 35-3 
Lycana scudderi. Liw.

1x78. Larva. Sounders. Canul. Fintom., vol. 10, p. 11.

1х80. Larva. Miss Middleton, (quotes Samuders.) 'Trans. Dept. Agr. III, vol. 18, A pendl., p. 95.

Foon I'Laxt. Lupinhs perennis.

Lycana acmon. Doubl.-llew.

1875. Latrva. Hy, Lidwartls. P'roc. Cal. Acall. Sc, April, (as L. antargon.)

Foud l'baxt. Hosuchio.

Lycana pseudargiolus. IBois.-Jec., (includ, vars.)

17:7. Latru, chrys. Ablot-Smilh. Lep. Ins. (ieorgia.

18333. Latria, chrysalis. Boistluval-Leconte. Hist. Iep. N. Amer., p. 118.

1xt:2. Latra, ehrysalis. Morris, (‘uotes Bois.-Lee.) Synop. Lep. N. Amer., p. 82.

istix. Lanva. Sunuder. Canad. Entom, vol, 1, 1. 100, (an I. neglecta.)

1869. Lailva, (col'd tig.) H. H. Eilwarle, (quotes Abb.-Sm., Bois.-Lec.) lintt.

N. Amer., 1st series, pl. Lyeir'na, No. 2.

1673. Eggu, larval stages. W. II. Eilurarly. Cnnad. Entom., vol, 5, p. 221.

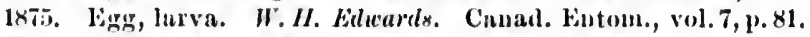

16ix. Lifo history. W. II. Eilwards. Cimat. Entom., vol.10, p. 12.

1881). Jallva. Mirs Middleton. T'rans. Dept. Agr. Ill., vol. 18, Appead., p. 95.

1880. Jarva. Miss Midlleton. Loc. cit., p. 96, (as L. neglecta.)

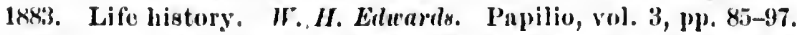

1881. Lifo history, (col'd figs.) I'. H. Eklwurly. Butt. N. Amer., 2al series, pl. Lyerina, No. 2.

1881. Larva, chrysilis. Feruald. Butt. Matine, p. 89, (as L. negleetu.)

1886. Life history, (condensed.) French. Butt. East. U. States, p. 289.

Foud I'asts. Actinomeris, Cornus, etc.

Lycæena amyntula. Bois.

1881. Lifo history. W. G. Wright. Papilio, vol.4, p. 126.

Foof, J'LaNT. Astragalus.

Lycæna comyntas. Godt.

1833. Larva, chrysalis, (eol'il tigss.) Boistural-Leconte. IIst. Lop. N. Amer., p. 120, pl. 36.

186:. Larva, chrysalis. Morrix, (qnotes liois.-I.ec.) Synop. Lep. N. Amer.,p.83.

1862. Larva. Ilurris. Ins. Inj. Vegetat., Flint's edit., p. 275.

1869. Larra, chrysalis. IIarig. Entom. corres., p. 275.

1876. Lifo history. W. H. Elacarts. Ciunal. Entom., vol. 8, p. 202.

1880. Larva. Miss Middleton. Trans. Dept. Agr. Ill., vol. 18, Append., p. 95.

1881. Eggg, larva. Frnall. lintt. Maino, p. 92.

1886. Lifo history. French, (quotes W. II. Edwards.) Butt. East. U. States, p. 292.

Food Plants. Lespedeza, Phuseolus.

Carterocephalus mandan. Edw.

1888. Egg, larva. J. Fletcher. A Trip to Nepigon, p. 12.

Ancyloxypha numitor. Fab.

1862. Clarysalis, (brief.) Harris. Ins. Inj. Vogetat., Flint's edit., p. 308.

1880. Chrysalis. Miss Middleton, („uotes IIarris.) Trans. Dept. Agr. Ill., vol. 18, Append., p. 96.

1886. Chrysalis. French, (quotes IIarris.) Butt. East. U. Statos, p. 301.

Pamphila zabulon. Bois.-Lee., (incl. vars.)

1868. Egg, young larva. Sannders. Canad. Entom., vol. 1, p. 66.

1880. Larva. Miss Middleton. 'Trans. Dept. Agr. Ill., vol. 18, Appond., p. 97, (as P. lsobomok.)

1881. Egg, larva, (figs.) Scudder. Butt., their structure, etc., pp. 7,23.

1884. Egg, larva, (figs.) Fernall. Butt. Maine, p. 95.

1886. Egg, larva, (figs.) French. Butt. East. U. States, p. 304.

Foon I'LANT. Grassis. 
Pamphila phylaus. Drury.

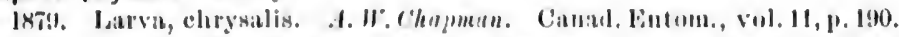

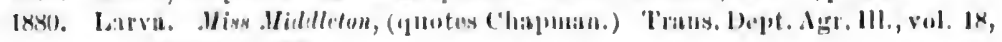

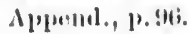

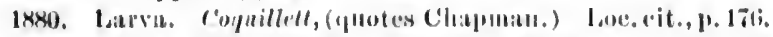

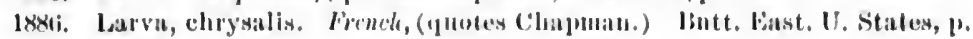
311.

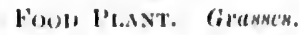

Pamphila brettus. Ibois.-1,e.

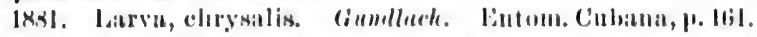

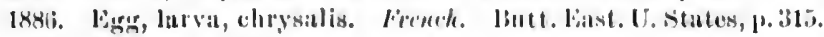

Foun Plast, firmane'x.

Pamphila otho. Livis.-lace.

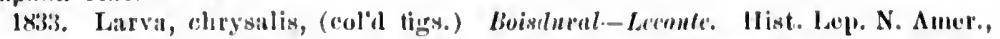
11. 17 . .

Pamphila mystic. Scud.

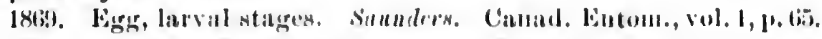

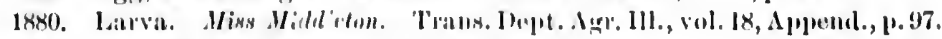

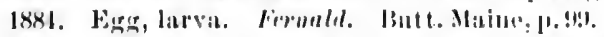

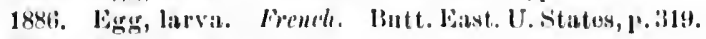

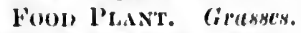

Pamphila sassacus. Harris.

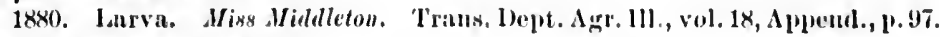

Pamphila cornes. Bois.-1.ee.

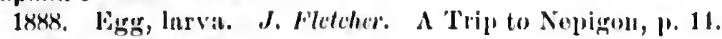

Pamphila peckius. Kby.

18lis. Egag, young larva. Sanuders. Camat. Entum., vol. 1, l, bit.

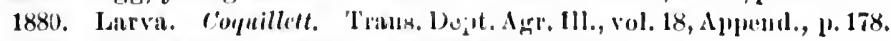

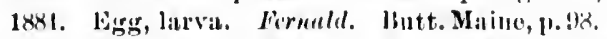

1886. Eirg, larva. Finuld. Butt. East. U.Stales, p.318.

Fool Plast. Grassis.

Pamphila accius. Al,b.-Smitl.

1879. Larva. A. II. Chapmen. Camal. Fntom., vol.11, p.\$1.

1881. Clirysalis, (fig.) Scudder. Butt, illeir structure, otc., 1.42.

Pamphila maculata. Eis.

1879. Larva. A. IT. Chapmen. Catual. Eintom., vol. 11, 1. 191.

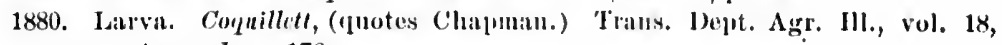
Append., p. 176.

1886. Larva, chrysalis. French. Butt. Vast. U. States., 1. 330.

Pamphila cthlius. Crain.

1877. Latrva. C. R. Modyge. Field and Folest, vol. 3, p. 73.

1881. Larva, elnysalie. Gundlach. Eutom. Cubana, 11. 1to.

1887. Chrysalis. Hy. Lelwarly. Lintom. Aner., vol. 3, 1. 163.

Pamphila arpa. Buis.-Lec.

1883. Larva, elnysalis, (figs.) Boisturul-Leconte. Ilist. Le?. N. Amer., pl. 68.

1879. Latra, chrysalis. A. W. Chepmen. Camat. Eutom., vol, 11, 1. 191.

1886. Larva, elurysalis. French, ('fuotes C'hapmin.) Butt. East. U. States, li. 339.

Food Plant. Suw-jalmetto.

Pamphila palatka. Elw.

1883. Larva, chrysilis, (tigs.) Boisdutal-Leconte. Ilist. Lep. N. Anter., pl.67, (as P. bulenta.)

1879. Larva. Chapmat. Canad. kintom., vol. 11, 11. 192.

1880. Larva. Coquillett, (quotes Chapman.) Trans. Dept. Agr. Ill., vol. 18. $\Lambda$ ppend., p. 15 I. 
1886. Larval. French, (4notes Chapman.) Butt. Eisst. U. States, p.ïl1.

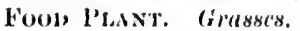

Pamphila delayare. Lilw.

1879. Larva, chrysalis. A. W. Chapman. Cantul. Eutoun, vol, 11, 1. 193

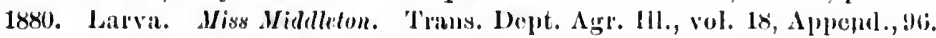

1880. Latra. Cormillett, (quotes Chapmian.) Loc. cit., p. 171.

1866. Lalfa. French, (yuotes Chapuim.) Butt. East. U. Stattes, p. 313.

Food l'Lavi'. Grusse's.

Amblyscirtes vialis. Edw.

1888. Ergr, larva. J. Flleteher. A Trip to Nepigron, p. 15.

Nisoniades brizo. Jois.-Lee.

1833. Lanva, chrysallis, (ligs.) Boistarel-Leconte. Ilist. lap. N. America, pl. 6 b.

Nisoniades icelus. Lintn.

1869. Eurg. Linturr. 3il liept. N. Y. State Cab. N. IIist., ]. 163.

1885. Latrva, chrysalis. II. II. Edwards. Canat. Entom., vol. 17, p. 98.

1886. Eggr. Frmelh, (1notes Lintner.) Butt. Bast. U. States, 1. 355.

1888. Egg. J. Fleteher. A Trip to Nepigon, p. 15.

Foon Plant. Populns tremuluides.

Nisoniades lucilius. Lintn.

1870. Jarva. Lintner. 24th Rept. N. Y. State Cal). N. Hist., p. 165.

1875. Egg, young larva. T. L. Metal. Canad. Entont., val. 7, p. 163.

1×78. Lile history. Lintuer, Entom. Contrib., No. 4, p. 1i7.

1880. Larva. Miss Middleton. 'Trans. Dopt. Agr. 1ll, vol. 18, A pluend., 1. 97.

1886. Egrg, larva, chrysalis. Fonch. Butt. East. U. States, p. 3\%9.

Foon Prast. Aquileyia canudensis.

Nisoniades persius. Send.

1881. Nest, (fig.) Sculler. Butt., their structure, etc., p. 108.

Nisoniades juvenalis. Fal.

1797. Larva, chrysalis, (col'll figs.) dbbot-Smith. Lep. Ins. (ieorgia, pl. 21.

1833. Larva, chrysalis, (col'd figs.) Boistncul-Lceontc. Ilist. Lep. N. Amer., 13. 65.

1862. Larva, chrysalis. Ifarris. Ins. Inj. Vegotat., Flint's clit., 1. 309.

1880. Lalva, chrysalis. Miss Middleton. 'T'rans. Dept. Agrr. 11l., vol. 18, Append., p. 97.

1881. Larva, chrysalis, (firss.) Scudder. Butt., their structure, etc., 1. 11.4, (ats Than, ennius.)

1886. Larva, chrysalis, (brief.) Freneh, (quotes IIarris.) Butt. East. U. States, p. 364 .

Food Ptants. Lathyrus, Apios, ete.

Pholisora catullus. Fab.

1797. Larva, chrysalis, (col'l figs.) Abbot-Smith. Lep. Ins. Georgia, pl.24.

1880. Larva. Miss Midllleton. 'Trans. Dept. Agr. IHI., vol. 18, Alpend., p. 98.

1881. Egg, (fig.) Scnelder. Butt., their strueture, otc., p. 9.

1885. Life history. W. H. Eilicarls, Cituad. Eutom., vol. 17, p. 245.

1886. Egg, (fig.) French. Butt. East. U. States, 1. 367.

Food Piants. Monardı, Cisenoporlium, Ambrosia.

Eudamus pylades. Scud.

1881. Egg, (fig.) Seudder. Butt., their structure, otc., 1. 6.

1884. Egg, (fig.) Fernald. Butt. Maine, p. 104.

1886. Egg, (fig.) French. Butt. Eist. U. States, p. 369.

Eudlamus bathyllus. Abl.--Suith.

1797. Larva, clurysalis, (col'd figs.) Abbot-Smilh. Iop. Ins. (ieorgia, pl. 22.

1833. Larvu, chrysalis, (col'd figs.) Boisdurul-Leconte. Hist.Lep. N.Amer., pl. 74.

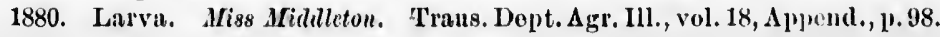


Eudamus lycidas. Al,wot-Sinith.

1797. Lallva, chrysalis, (col's tigss.) Ahlot-smith. Lep. Ins. (ieorgia, pl. 20.

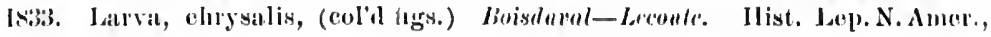
pl. 71.

Eulamus ce!lus. Bois.-Lee.

1633. Larva, "hrgsalis. Iosismural-Leoute. Ilist. Lep. N. Amer., pl. 73.

Eudanus tityras. Fals.

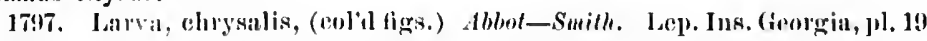

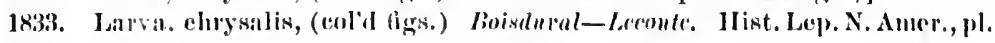
i:.

1841. Iarvi, (luysalis. IItris. Ins. Inj. Vegetat., 1st elit., p. 221.

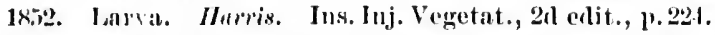

18il. Laria, chrysalis, (eol'd tigss.) E'mmons. Nat. Ilist. N. York, pl. 8:32.

1858. Larvil. Filch. 5th Rept. Ins. N. York, \$330.

1859. Larva, nest, elorysilis. I'. H. Gosse. Let ters from Alabama, p. Ka.

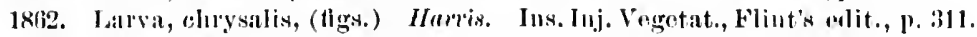

1862. Lavia. Mowis, (quotes Ilarris.) Synop. Lej. N. Aner.,p. 112.

18iT. Larva. Fromeh. 'Trans. Dept. Agr. III., vol. 15, p. 163.

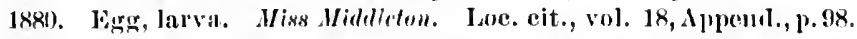

18x0. Larva. Compillett. Loc. cit., vol. $1 \times, \lambda$ ppend., j. 153.

18x1. Nest, larra, chrysalis, (figs.) Sculder. Butt., their structure, etc., p. 107.

1881. Cocoon, chryalia, (firs.) simdler. Ioc. cit., p. 2iti.

1881. Lanra. Pachard, (quotes Ilarris.) Ins. Inj., Forest Trees, p. 100.

1885. Larvit. II!. Edwards. Kingsley's Stand. Nat. Hist., vol.2, p.475.

1886. Chrysalis, (fig.) Frueh. Butt. East. U. States, p.376.

188ti. Life history. French. Loe, eit., p. 375.

Fool, P'sters. Rubinia, Acocin, Mistaria.

Eudamus protcus. I.

17!17. Larwa, chrysalis, (col't figs.) Abbot-Smith. Icp. Ins. (icorgia, pl, 18.

1833. Larva, chrysalis, (col's figns.) Boistlural-Lecoute. IIist. Lep). N. Amer., pI. 69 .

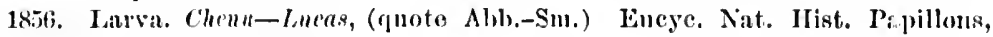
vol. 1, p. 223.

1879. Larva, chrywalis. A. W. Chapman. Canar. Entom., vol. 11, p. 173.

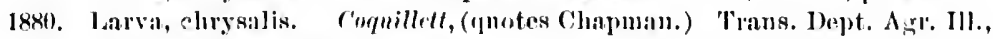
rol. $18, \Lambda_{\text {plpend., j. } 181 .}$

1881. Larva, elurysalis. Fimellech. Eutom. Culana, p. 169.

1886. Larva, chyysalis. French, (ynotes Chapman.) Butt. Bast. U. States, p. 378.

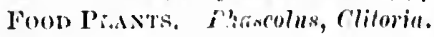

Megativerus yanne. isis-bee.

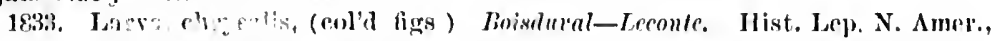
fi. - i, rus imdamms Yucees.)

187t. Larva, chrysalis, (ligss.) Jiley. Trans. Acal. Se. St. Lon is, pp. 323-31.

18i6. Lifo history, (firs.) Riley. Xth Missonri Rept., pl. 108-183.

1877. Life histony, (tigs.) Rilly. Oth Missomyi Rapt, p. 12!.

18*6. Lifo history, (figs.) French, (ynotes Riley.) IBntt. East. U. States, j. 383.

Poob Plane. Stems of Fucea.

Meyathymus cofaqui. Strecker.

187i. Larva. Strecler. Proc. Acat. Nat. Se. Philah., p. 148.

\section{HETEROCERA.}

\section{SPHINGIDA3.}

Hemaris tenuis. Cirote.

1. 22.

1877. Ialva, G,o/c. Psỵelw, vol. 2, p. 66.

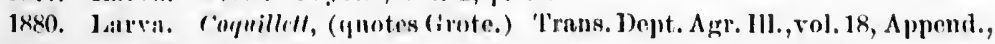
p. 160. 
1883. Larva. Fh. Fixther. Canad. Lutum., vol. 15, p. 2as.

18xt. Eign, larvil. I'l. Fischer. Canal. lintom., vol. 16, p. 113.

1886. . Larva, pupa. W. J. IJollund. Canad, Lintom., vol. 18, p. 101.

Food Plavis. Symphoricarpus racemosus.

Hemaris marginalis. (irote.

Life history. II. S. Jewetl. Bull. Brooklyn Entom. Soc, vol, 4, p. 17.

Foon Plantr. Lonicera semperimens.

Hemaris diffinis. lioris.

1797. Larva, (col'l lig.) Albot-Smith. Lep. Sus. (ieorgia, p. 85, pl. 49

1839. Larvi. Harris. Sillimau's Jomr. Sc. Art., vol. 36, p. 308.

18:9). Larva. Clemens. Jomrual Aeall. N. Se. Philad., (July.)

1862. Larva, (brict.) Morris, (ynotes Clemens.) Synop. lap. N. Amer., p. 14!.

1870. Larva. T. L. Meul. Canarl. Entom., vol. 2, p. 157.

1870. Larva. Lintuer. 24th Rept. N. Y. State Calo. N. Hist., p. 110.

187.. Larva. Boisiluetl. Spee, Gener. Sphingrida, p. 367.

1880. Larva. Coquillett. 'Trans. Dept. Agrr. 1ll., vol. 18, Append., p. 160.

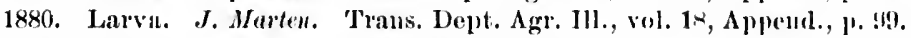

1882. Larva. E. B. Reed. 12th Rept. Butom. Soc. Ontario, p. 50.

1886. Larva. Fernalit. Sphingidae N. Engl'd, p. 15.

Food Plants. Apoeymum, Symphoricarpus.

Hemaris thysbe. Fab., (inclucl. vars.)

1864. Larva, pupa. Lintuet. l'roc. Entom. Soc. Philat., vol. 3, p. 616.

1874. Larva. Boiselural. Spee. Gener. Sphingidir, 11. 370.

1879. Larva, pnpa. (i. I). Hulst. Iull. Brooklyn Entoun. Soc., rol. 2, p. 38

1880. Larva. J. Marten. 'Trans. Dept. $\Lambda$ gr' Ill, vol. 18, $\Lambda$ ppent., p. 99.

18x2. Larva. E. R. Reel. 12th Rept. Eutom. Soc. Ontario, p. 51.

1883. Larva, pupa. Ethords-Elliot. Papilio, vol.3, p. 125, (as H. uniformis.)

188.5. Larva. Fermald. Kingsley's Staml. Nat. IList., vol. 2, 1. 46.5.

1826. Larva. Fermall. Splingride N. Mugl'd, p. 17.

1888. Lanrva. J. B. Smilh, ('qnotes Liv unor-ILulst.) Monog. Sphing., p. 100

Foon Plasts. Tiburnum, Crateryus, l'rumus, ete.

Hemaris buffaloensis. G. and $R$.

1870. Life history. Lintuer. 21th Rept. N. York State Cals. N. Ilist, p. 11 .

1888. Larva. J. B. Smith, (đuotes Lintuer.) Monog. Spling., p. 10\%.

Foon Plant. Tihmrmmm.

Hemaris etolus. B.is.

187. Larva. Boisthval. Spec. (iener. Sphingidlip, p. 370.

Foon Plaxt, riburnum.

Hemaris pyramus. Bois.

1874. Larva. Iboiselural, (1untes Lcente.) Spee. Goner. Sphingidir, p. 372.

food Plant. Jihumum.

Ellopos tantalus. L.

1887. Pupa. Hy. Edlwards. Fntom. Amer., vol. 3, p. 163.

Cautethia grotei. IIy. Flwards.

1881. Larva. Gundlaeh. Entom. Culıana.

1887. Pupa. Hy. Edwards. Entom. Amer., vol. 3, p. 164.

Food P'ant. Rubinceg.

Pogocolon gauræ. Abb.-Sm.

1797. Larva, pupa, (fig.) Ahbot-Simith. Lep. Ins. (ieorgia, p. 61, pl. 31.

18:9. Larva. Clemens. Jour. Acaul. N. Se., I'hilad.

1862. Larva, pupa. Morrix, (qnotes Ah.-Sw.) Syuop. Lep. N. Amer., p. 1.1.

1874. Larvn. Boistural, (guotes Alb.-Sm.) Spee. (iener. Splinginlir, 1. 315.

Food Plant, Genra hienuis.

Amphion nessus. Cramer.

187.4. Larva, (col'd fig.) Boistluval. Spee. Gener. Sphingidir, p. 817, pl. 1.

1877. Latru. W. I. Aulrews. Canal. Eutom., vol. 9, p. 19. 


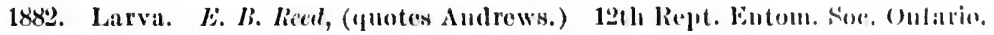
188:. Larva, brief. Julia E. sianders. P'apilio, vol, 2, p. 147.

1886. Larva. Fernuld. Spluingidie N. Engl'd, p. 21.

Eoon Plants. Rubiacel', Jitig.

Thyreus abbotii. Swainson.

18339. Larva. Jarris. Silliman's Jour. Se. Art, vol. 36, p. 307.

1856. Larva, (lig.) Chenu-Drmarets. Eneye. Ilist. Nat. Papillons, vol. 1, p. המ.

18̄59. Larva, pupa. Clemens. Jour. Acad. N. Sc. Philad., July.

186i2. Larva, pupa. Morris, (unotes Clemens.) Synop. Lep. N. Amer., p. tifi.

1869. Larva. Harris. Entom. Corres., p. 284.

1870. Larva, pupa. Lintuer. 24th Rept. N. York Statto Cah. N. Ilist., p. 111.

1874. Larva, pupa. Boistural. Spec. Gener. Sphingidal, p. 331, (firm dhiot 's (lrawing.)

1876. Larva. C. P. Whitney. Canad. Entom., vol. 8, p. 75.

1876. Larva, (brief.) A. R. Grote. Camad. Entom., vol. 8, p. 100.

1877. Larva. Freneh. Trans. Dept. Agr. Ill., vol, 15, p. 164.

1878. Larva, (fig.) G. H. I'erkins. 5th Rept. Vermont Agr. Soe, p. 281.

1878. Larva, (fig.) Saunders. Canad. Eutom., vol. 10, p. 130.

1879. Lifo history, (figs.) Riley. 2d Missouri Rept., p. 78.

1880. Larva, (fig.) Saunders. Rept. Entom. Soc. Ontario, p. 42.

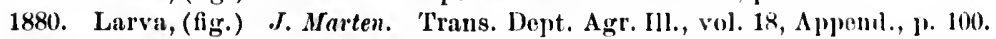

1881. Larva, (fig.) Saunders. Canad. Entoun., vol. 13, p. 2.

1882. Larva, (fig.) E. B. Reel. 12th Rept. Entom. Soc. Ontario, p. 5i3.

1882. Larva, (brief.) Julia E. Saunders. Papilio, vol. 2, p. 117.

1883. Larva, pupa, (figs.) Saunders. Ins. Inj. Fruits, p. 253.

1886. Larva, (fig.) Frmalit. Sphingidie N. Engl'd, p. 23.

Food Plaxts. Titis, Ampelopsis.

Enyo lugubris. 1 .

1797. Larva, pupa, (fig.) Abbo'-Smith. Lep. Ins. Georgia, 1. 59, pl. 30.

1837. Larva, pupa. Westwooll, (quotes Abl.-Sm.) Drury's Illus. Exot. Entom., vol. 1, p. 56.

1839. Larva. Harris, (quotes Abb.-Sin.) Silliman's Junt. Se. Art, vol, 36, 1). 307.

1859. Larva. Clemens. Jour. Acad. N. Sc. Philad., July.

1862. Larva. Morris, (quotes Abb.-Sur.) Synop. Lep. N. Amer., p. 162.

1874. Larva. Boistuval. Spec. (iener. Splingidin, p. $2: 77$.

Foon Plant. Ampelopsis hederaeca.

Deidamia inscripta. Harris.

1862. Dnpa. Clemens. Morris's Synop. Lep. N. Amer., p. 159.

1877. Larva, pupa. Strecke', (fide Akburst.) Rlıpral. et IIteteroe., p. 112.

1886. Larva, pnpa. Frrnald. Sphingidie N. Engl'd, p. 70.

Fool Plant. Ampelopsis.

Deilephila chamænerii. Harris.

1839). Larva. Harvis. Silliman's Jonr. Se. Art, vol. 36, p. 305.

1859. Larva. Clemens. Jour. Acad. N. Se. Philitd., July.

1862. Larva. Morris, (qnotes Clemens.) Symop. Lep. N. Amer., p. 16i.

1864. Larva, pupa. Lintuer. Proc. Entom. Soc. Plitaul, vol. 3, p. fibl.

1870. Larva, pupa, (brief.) G.J. Bowl's. Canall. Lintom., vol.:3, p. 1.1\%.

187.4. Larva. Boisduval, (as D. galii.) Spec. Goner. Sphingrida, p. 170.

1876. Larva, (fig.) Suunders. Rept. Entom. Soc. Onturio, p. 37.

1877. Larva, (fig.) Saunders. Canad. Entom., vol.9, p. 6it.

1880. Lurva. Coquillett, (quotes Sanuders.) 'Wrans. Dept. Agr. Ill., rol. 18, Append., p. 159.

1882. Larva. E. B. Leerl, (quotes Sannders.) 12tlı Rept. Enton. Soc. Ontario, p. 54.

1883. Larva, cocoon, pupa. Saunders. Ins. Inj. Frnits, p. 257. 


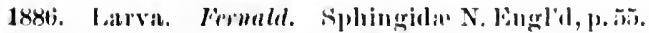

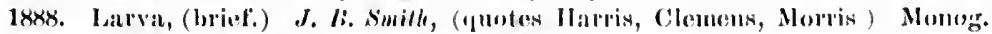
spling., 1). 133.

Food Plant. Epilobium angustifinlium.

Deilephila lineata, Falrs.

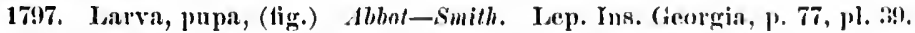

1828. Jarva, pnpar. Stephens. Illus. Brit. Entom., Hanst., vol. 1, p. 127.

1839. Lawa. Harris. Silliman's Jomr. Se. Art, vol. 36, p. :301.

18:6, Jarva, pma. Chomw. Encyc. Ilist. Nat. Japillons, p. 2ti2.

18:9. I,arva, pupa. Clemens. Iour. Acal. N. Sc. Jhilaul., July.

1860. Latva. Dnnean. Natural. Library, vol. 30, 1\%. 15\%.

1862. Intva, pupa. Norris, (quotes Clemons.) Synop. 1.ep. N. Amor., p. 161.

186t. Larva, pupa. Limtuer. I'roc. Futoun Soc. I'hilad., vol. 3, p. (ifi2.

1871. Jarva, (fig.) libley. Bd Missouri Rept., p. 1 H.

1874. Larva, (lig.) Sannders. Rept. Entom. Soc. Ontario, 1. Qx.

1874. Jarva. Boiselural, (quotes Abbot as 1). dancus.) Spee. (iener. Sphingrilar, 1). 171.

187. Layva, (fig.) .J. G. Hood, (as D. daucus.) Ins. Aloroad, p. (j37.

1875. Larva, pupa. Hy. Edwards. Proc. Cal. Mead. Se.

1876. Lavra, (fig.) Sammles. Rept. Lintom. Soo. Ontario, 1. 37.

1877. Larva. French. 'Trans. Dopt. Agr. 11l., vol. 15, p. 16il.

1877. Larva, (fig.) Stunnders. Canaıl. Eutom., vol. !), p. (i).

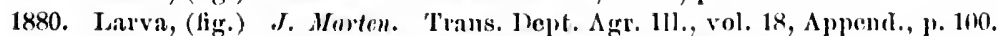

1880. Iarva, (fig.) Coquillett. 'Trans. Dept. Aerr. Ill., Append., vol. 18, p. 159.

1882. Larva, (figr.) Li. B. Reel, (puotes liluy.) 12th Rept. Eutum. Soc. Ontario, p. 5is.

1883. Lavva, pupa, (ligs.) Sanmers. Ins. Inj. Fruits, p. 25\%.

1886. Larva, (fig.) Fermald. Sphingidar N. Enerlil, p. 5T.

1886. Larva, pmpa. Gumbllach. Eutom. Culsuma, p. 196.

Food Plants. Pontuluca, Pyruts, Fuelesia, rte.,

Philampelus vitis. Drury.

1705. Larva, pupa, (eol'd ligs.) Merim. Metam. Ins. Surinam, 11. 17, pl. 47.

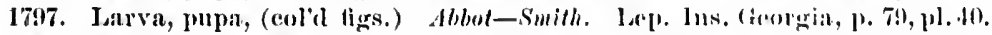

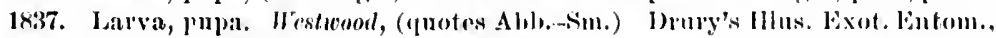
vol. 1 , 1). 5\%.

1839. Larva. Marris, (quotes Alu.-Sm.) Silliman's .Iour. Sc. Art, vol. 3(i, p. 299.

1858. Ianva, pupa, (col'd tigs.) Duncun. Natural. Tibrary, vol, 2"-, p. 105, pl. 7.

18:9.). Lavva, pupa. Clemems, (quotes Alb.-Sm.) Jomr. Acar. N. Se. Philad., Jixly.

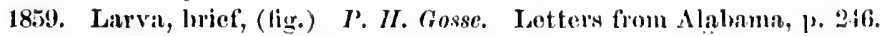

1862. Iarva, pupa. Morris, (quotes Clemens.) Synop. Jep. N. Amer., p. 17 (1).

1886. Larva, pupa. Gunllach. Jintom. ('nb:ina, p. 18!).

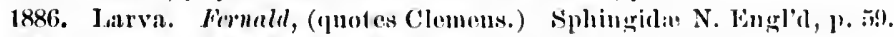

1887. Larva. My. Eilwarls. Entom. Amor., vol. 3, p. 16.

Foon P's an'r. Jussiand decurvens.

Philampelus pandorus. IIibu.

1705. Iarva, pupa, (col'd figrs.) Morion. Metam. Ins. Sminam, 1. 47, pl. 47.

1839. Larva. Harris. Sillimin's Jomr. Sc. Art, rol. \$(i, 1. 300, (as I'. saldellitia.)

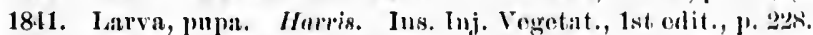

185.t. Lnvin. Emmons. Nut. Ilist. N. York, vol. 5, p. 220.

1836. Larva. Fïth. 3d lept. Ins. N York, p. 39!8.

1859. Larva. Clemens. Jour. Arat. Se. Philat., July.

1862. Larva. Morris, (quoles Chmens.) Symop. I.op. N. Amer., p. 177.

1861. Larva, pupu. Lintner. Proc. Entom. Soc. Philad., vol, 3, p. firs.

1861. Larva. Jacy'r. Idifo N. Amer. Ins., p. 193. 


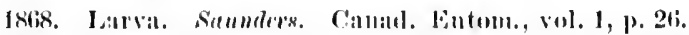

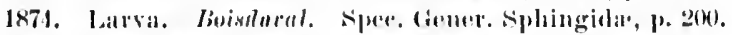

1876. Lifo history, (figrs.) libe!. 2ol Missonri liept.

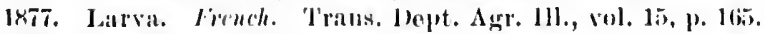

1877. Intra. Scmider. Psyolhe, vol. 2, p. tix.

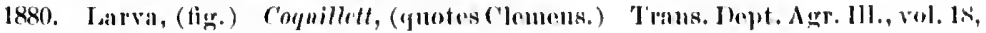
Alpend., p. 17\%.

18xt. Lairval stages, (figs.) .J. Wurten. 'Trans. Hept. Agr. Ill, Append., vol. Ix, 1). 101.

18\$1. Larva. Sinuler.x. Canal. Entom., rol. 13, p. 41.

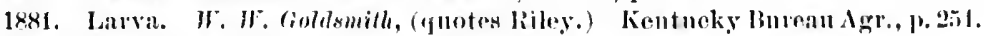

18*2. Larva, (fig.) Sumders. 12tl Rept. lintom. Soc. Ontario, p. 19.

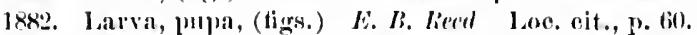

1883. Iarva, pupa, (tig.s.) Sanuders. Ins. Inj. Finits, p. 2t!.

1886. Larva, (fig.) Feruald. Splii. itla N. Eingl'd, p. 60.

188ti. Larva, (hrief.) Juliu E. Saumders. I'apilio, vol. 2, 11. 117.

1888. Larva, pupa, (ligr.) Grote. 18th Rept. Entom. Soc. Ontario, p. 73.

Foon Pr.axts. Ampelopis, ritis.

Philampelus achemon. Drury.

1797. Lalva, (col'd firr.) Ablot-Smith. Lej). Ins. (ieorigia, p. 81, pl. 11.

1839. I.arva. Herris. Silliman's Jomr. Se. Art, vol. 36, p. 3(1).

1841. Larva, pupa. Ilarvis. Ins. Inj. Vegetat., 1st edit., p. 228.

1850. Jarval stages. Clemens. Jour. Aead. X. Se. Jhilnd., July.

1862. Iatrval stalires. Morris, (1)otes Clemens). Symop. lep. N. Anor., p. 17x.

1862. Lalva, puph, (figs.) IJaris. Ins. Inj. Vegotat., l'lint's edit., p. 325.

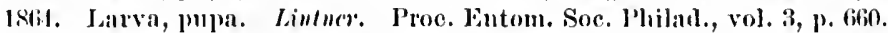

1869. Iarva, (fig.) Harris. Eutom. Corres., pl. 111.

1870. Wers, larval stages. Limture. 2fth Rept. N. York State Cal, N. Ilist., p. 116.

1xit. Jarva. Boisdural, (yuotes Lorquin in lit.) Speo. (iener. Sphingrilar, p. 200.

tsit. Lito history. Rily. 2d Missonri Rept., p. 74-76.

1877. Iarva. French. 'Trans. Jept. Agr. Ill., vol. 15, p. 165.

1878. Iatra, pupa, (fig.) Sanuders. (amad. Eutom., vol. 10, p. 103.

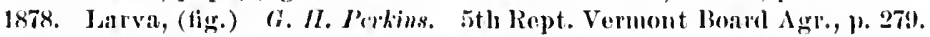

187!!. Iansa, pupa, (tigs.) Sunulers. Rept. Entom. Soe. (hutario, p. 7.1.

1880. Larvi, (fig.) J. Harten. 'l'rans. Dept. $\Lambda$ grr. Ill., vol. 18, Append., p. 101.

1880. Iarva, (figr.) C'ounillett, (guotes Clemens.) Trans. Dept. $\Lambda$ gr. Ill., vol. 18, Append., p. 182.

1882. Latva, (brief.) Julia E. Stmulers. I'apilin, vol. 2, p. 117.

1883. Isarval stages, pupa, (figs.) Sammelors. Ins. Inj. l'puits, p. 251.

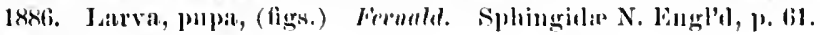

Food Praxt. Jitis.

Argeus laibruscæ. I.

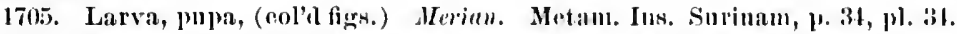

171!. Larva, (col'd tig.) Matame Meritu. Ins. of Surinam, pl. 31.

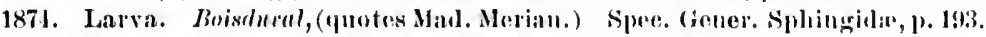

1886. Jalva, (brief.) Gumllarh. Entom. Cubana, p. 187.

Fo(i) Phant. Cissus.

Pseudosphinx tetrio. L.

1832. Larva, (ligr.) Pocy. Lep. Cubana.

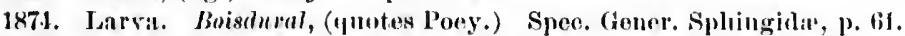

1878. Jurva, pupa, (ligs.) H. Meritz. Wiegemann Alehiv. Natulumseh., p. 9, pl. 1.

1886. Larva, pupa. Gundluch. Eutou!. Cuhana, p. 201.

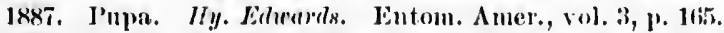

1887. P'ipa. IIy. Eilmards. Camad. Entom., vol. 20, p. 14.

l'von l's.a.tes. I'lumirera rubro. .Taminimu. 
Pachylia ficus. I.

1719. Larva, (fig.) Mleeteme Merian. Ins. of Suriuam, pl, 33.

187.1. Lurva. Boistherd, (qnotes Mad. Merian.) Spece Gener. Sphingidae, p. 137.

188.1. Larva, (brief.) W. S'chaus, jr. Papilio, vol. 4, p. 21.

1886. Larva, pupa. Gundlach. Entom. Culsana, p. 201.

1888. Larva, (hrief.) J. B. Smith, (quotes Fab., Merian, Boisd.) Monogr. Spling., p. 139.

Food Plant. Fichs.

Chœrocampa tersa. L.

1797. Larva, pupıa, (col'd fig.) Abbot-Suith. Lep. Ins. Goorgia, p. 75, pl.38.

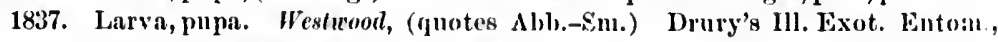
vol. 1, p. 56 .

1839. Larva, pupa. Harris, (quotes Ab,.,-S!̣n.) Silliman's Jour. Se. Art, vol. .... p. 303.

1858. Larva, (col'd fig.) Duncan. Natural. Library, vol. 22, p. 100, pl. 6.

1859. Larva. Clemens. Jonr. Acad. N. Sc. Philad., July.

1862. Larva. Morris, (quotes Clemens.) Synop. Lep. N. Amer, p. 172.

187.4. Larva, pupa. Boisdural, ('notes Abhot.) Spec. (iener. Spllingido, p. sfi.).

1880. Larva. Coquillett, ('unotes Abb.-Sm.) Trans. Dept. Agr. Ill,, vol. Is, Append., p. 159.

1882. Larva. E. B. Reed. 12th Rept. Entom. Soc. Ontario, p. 56.

1886. Larva. Ferutld. Sphingidar N. Engl'd, p.63.

1886. Larva, pupa. Guellach. Entom. C'nlana, ]. 192.

1887. Pupa. IIy. Edturds. Entom. Aner., vol.3, p. 161.

Food l'lant. Rubiacere.

Everyx chœrilus. Bdv.

1797. Larva, pupa. Abbolt-Smilh. Lep. Ins. Georgia, p. 53, pl. 27.

1839. Larva. Haris, (quotes $\Lambda$ b).-Sin.) Silliman's Jonr. Se. $\Lambda$ rt, vol. 36, p. 302.

1859. Larva, pupa. Clemens, (qnotes Ahb.-Sm.) Jour. A tad. N. Se. Philud.

1862. Larva, pupa. Mowris, (quotes Clemens.) Synop. Lep. N. Amer., p. 168.

1869. Pnpa. IIarris. Fintom. Corres., p. 283.

1874. Larva, pupa. Boistlual. Spec. Gener. Sphingidie, p. 211.

1882. Larva. L. B. Reel, (quotes $\Lambda$ bb.-Sin.) 12th Rept. Euto!n. Soc. Ontario, p. 57.

1883. Larva, pupa. Edwards-Elliot. Papqilio, vol. 3, p.126.

1886. Larva, pupa. Formald, (quotes Edw.-Elliot.) Sphingida N. Eng'd, p. 6i. Foon Plants. Azalea, Tibumum.

Everyx myron. Cramer.

1797. Larva, (lig.) Ablot-Smith. Lep. Ins. Georgia, p. 55, pl. 28.

1839. Larva. Harris. Silliman's Jour. Sc. Art, vol, 56, p. 302.

18.1. Larva, pupa. Harris. Ins. Inj. Vegetat., 1st edit., p. 229.

1852. Larva. Harris. Lus. Inj. Vegetat., 2łl edit., p. 249.

18:ti. Larva. Fitch. 3it Rept. Ins. N. York, p. 397.

1859. Larva, pupa. Clemens. Jour. Aearl. N. Se. Philarl., July.

1862. Larvn, pupa. Morris, (quotes Clemons.) Synop. Lop. N. Amor., p. 169.

1862. Larva, pupa, (fig.) IIaris. Ins. Inj. Vogetat., Flint's edit., p. 327.

1861. Larval stages, pupa. Lininer. Proc. Entom. Soc. Philad., vol. 3, p. 663.

1871. Larva, (fig.) Sumule's. Rept. Entom. Soe. Ontario, p. 357.

1871. Larva, pupa, (fig.) Saunders. Canad Entoul, vol. 3, p. 66.

1871. Lilo history. (figs.) Rilcy. 2d Missouri Rept., p. 71-73.

187.. Larva, pupa. Boisduval. Spee. Genor. Sphingide, p. 210.

1877. Larva. French. 'Trans. Dept. Agr. Ill., vol. 15, p. 163.

1878. Larva, (fig.) (r. H. Perkins. 5th Rept. Vermont Board Agr., p. 277.

1879. Larva, (fig.) Mis. Ballard. Insect Lives, p. 67.

1880. Larva. (fig.) .J. Mfarte'n. 'Trans. Dopt. Agr. Ill., vol. 18, Appenc., p. 10:3.

1882. Larva, pupa, (ligs.) E. B. Ried. 12th Ropt. Entom. Soc. Outario, p. 58. 


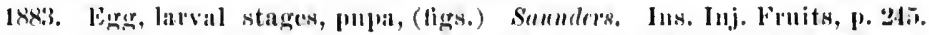

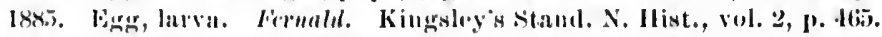

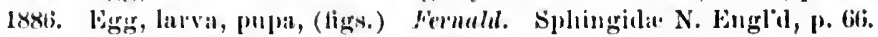

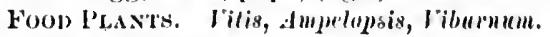

Ampelophaga versicolor. Ilarris.

18iti. Larva, (brief.) G. W. Peck, Canal. Entom., vol. 8, p. 239.

18ix. Life listory. G. 1). Mulst. Camal. Entom, rol. 11, 1. (BI.

1860. Larra. C'muillett, (cuotes Ilulst.) Trans. Dept. Agr. I11., vol. 18, Appent., p. 1:8.

1881. Larva, (brief.) Pl. Fischer. Bull. Bnllalo Soc. N. Sc., vol. 4, p. fi2.

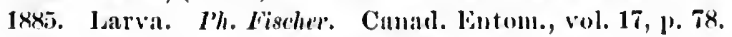

1886. Eugg, larval stagas, pupa. Fermuld, (quotes Ilulst.) Sphingidar N. Lugl'il, p. 68.

Foon Pluxt. Cephatanthus occillentalis.

Smerinthus ophthalmicus. Bois.

1869. Larva., Boistlural. Lep. Califor., p. 68.

187.1. Larva, (col'd tig.) pupa. Boisdnewl, (ynotes Lorquill in lit.) Spec. ficner. Spliningricler, p. 31, pl. 1.

1875. Eggr, larval stages. Hy. Eilucards. Proc. Cal, Acal. Se, $\Lambda$ pril.

Foun Pe.iNT. Sulix.

Snerinthus geminatus. Say.

1810. Larva, pupa. I'. II. Gosse. Camad. Naturalist, pp. 280, 295.

1870. Egrg, larval stages. Linturr. 2.th Rept. N. York Stato Cab. N. Ilist, p. 119.

187.. Larva. Boishluml. Spec. Gener. Sphingrider, p. 39.

188.. Larva. E. B. Reed. 12th hept. Eutom. Soe. Ontario, 1. 62.

1886. Egrge, larva. Fermall. Sphingidan N. Engl'l, p. Ts.

Foon l'laxis. I'rumus, Sulix, Betula, etc.

Smerinthus excæcatus. Al,b.-Sw.

1797. Larva, pupa, (figs.) Abhot-Smith. Lep. Ins. Georgia, 1. 49, pl. 2i.

1839. Larva. Harris. Silliman's font. Se. Art, vol. 36, p. 290.

1811. Larva, pupa. Marris. Ins. Inj. Vegetat., 1st ellit., p. 230.

1856. Latria. F'itch. 3i Rept. Ins. N. York, p.3ł.

18.59. Egrg, larval stages, pupa. Clemens. Four. Aead, N. Se. Pliilal., July.

186;. Nerg, larva, pnpa. Morvis, (quotes Clemens.) Synop. Lep. N. Amer., p.209).

1861. Larva, pupa. Lintuer. P'roe. Entoun. Soc. Philad., vol. 3, p. 66is.

1868. Larva. E. B. Reel. Cauad. Entonl., vol. 1, 1. 40.

18it. Larva, pupa. Boisdural, (quotes J. Leconte in lit.) Spee. (iener. Sphingrilia, p. 38, (after Abbot's irawing.)

1876. Larva. Strecker. Rhopal. et Ifeteroe., p. 55.

1877. Larva. I'ackind. Italf-hours with Insects, p. 180.

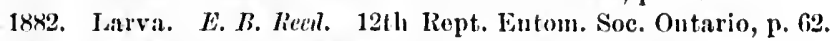

1883. Larva, (figs.) Sammaters. Ins. In,j. Fruts, p. 85.

188.1. Isarva, (figr.) Saunders. Canad. Entom., vol. 16, p. 11.

1885. Larva, (fig.) Saumlers. 15ith Rept. Entom. Soc. Ontario, p. 26.

1885. Larva. Fernald. Kingsley's Stand. N. Ilist., vol. 2, p. 468.

1880. Lava, pupa, (figs.) Firnall. Sphingidie N. Ingl'd, p. il.

Foon) Phines. Pyrus, Prunlls, Rosa.

Smerinthus myops. Abb.-Sm.

1797. Iarva, pupa, (figs.) Abbot-Smill. IJop. Ins. Georgia, p. 51, pl. 26.

1839. Larva. Haris, (quotes Abb.-Sin.) Silliman's Jour. Sc. Art, vol. 36, p. 291.

1859. Larva, pupa. Clemens. Jonr. Acad. N. Se. l'hilad., (after Abbot's figure.)

1862. Larva, pupa. Morris, (quotes Clemens.) Synop. Lep. N. Amer., p. 208.

1874. Larva, pupa. Boistural, (quotes J. Leconte in lit.) Spee. Gener. Splingidie, p. 42, (after Abbot's tignre.)

1876. Larva, (hric ' G. W. Peck. Canal. Entom., vol. 8, p. 239. 


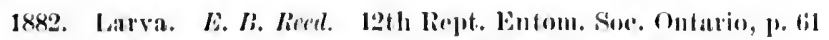

1883. Larva, pupa. Sonuders. Ins. luj. Frotits, p. 20x.

18xl. Larva. Saunders. Cantul. Bintom., vol. 16, p. 11.

1885. Latriat. Sannders. 15th liept. Entom. Soc. Outario, 11. 27.

18x6. Larva. Fomald. Sphingidle, N. Engl'd, 10.76.

18x6. Lava, L. Bunker. Canal. Jintom., vol. 18, p. 207.

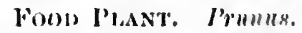

Smerinthus astylus. Drury.

187.1. Larvil. Boisdural, (quotes J. Leente in lit.) Spee. (ienel. Splingida, p. 41.

187f, Latra, (brief.) G. W. Prcli. Canad. Lintom., vol. 8, 1. 23:9.

1886. Larva. Formuld. Splingida, N. Engrid, p. 77.

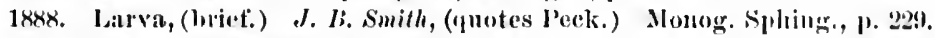

Foon Praxt. Viecinium corymbosum.

Triptogon modesta. IIarris.

1877. Lifo history. R. Bunler. Canad. Enlom., vol. 9, p. 210.

1877. Larva. W. V. Amlirws. l'syche, vol. 2, p. 72.

1880. Larva. Coquillst, (quotes Audiews.) Trans. Dept. Ayr. Ill., vol. 18, $\Lambda$ ppend., p. 159.

1881. Larra, pupal. D. S. Frllicolt. Bull. Buffilo Soc. N. Se, rol. 1, 1. 29.

1882. Larva. E. B. Rerd. 12th Rept. Entom. Soc. Octario, p. 6i3.

1886. Lifo history. Frmald, (4notes Bunker.) Splingidir, N. lingl'l, p. 72.

Foon Plasts. romlus, sulix.

Cressonia juglandis. Abl., Sin.

1797. Larva, pupa, (figs.) Abbot-Smith. Lop. Ins. Georgia, p. 57, pl. 29.

1839. Larva, pupa. Ilarris, ('notes Abl.-Sm.) Silliman's Jour. Sce, Art, vol. 36, p. 292.

1856. Larva. Fitch. 3t Rept. Ins. N. York, p. 462.

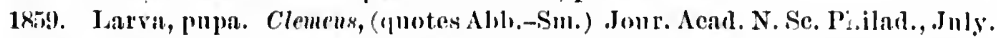

1862. Larva, pupa. Morris, (łuotes Clemens.) Synop. Lep. N. Amer., p. 213.

1861. Larva, pupa. Lintury. P'oe. Entom. Soc. Philad, vol. 3, p. 6i68.

1866. Larvi. IItrris. Entom. Corres., 1. 281.

1874. Larva, pupa. Boisdural, („uotes Abbot.) Spee. Gener. Sphingidie, p. 27.

1876. Larva, pupa. Strecker, (quotes $A$ bbot.) Rlopal. et IJeteroc., p. 54.

1881. Larva. I'aclard, (gnotes Harris.) Ins. Inj. Forest Trees, p. 81.

1882. Larva. E. B. Resil. 120th Rept. Entom. Soe. Ontario, p. tis.

1883. Young latrva. Edtrards-Elliol. l'apilio, vol. 3, p. 127.

1886. Lava, jupa. Frmuld. Sphingida N. Engl'd, p. 81.

Foon l'axts. Juglaus, Carya.

Ceratomia amyntor. IIiil)n.

1839. Larva. Ifaris. Silliman's Jonr. Sc. Art, vol. 36, p. 293, (as C. qualricornis.)

1841. Larva, pupa. Inaris. Ins. Inj. Vegetat., Ist edit., p. 227.

1852. Larva, pupa. IIaris. Ins. Inj. Vegetat., 2 l edit., p. 217.

1859. Larva, pupa. Clemens. Jonr. Aead. N. Se. Philat., July.

1862. Larvi, pupa. Morris, (quotes Clemens.) Synop. Lep. N. Amer., p. 20 5.

1862. I arva, (lig.) Hamis. Ins. Inj. Vegetat., Flint's edit., p. 324.

1862. Larva, (bricf.) Iackard. 2a Rept. Nat. Hist. and Genlog. Matine, p. 167.

1862. Egg, larval stages, pupa. Lintuer. Proc. Entom. Soc. Philal., vol. 1, p. 286.

1864. Pnpa. Lintuer. Loc. eit., vol, 3, p. 661.

1861. Larva. Jaeger. Lifo N. Amer. Ins., p. 192.

1869. Wggg. Minot. Canad. Entom., vol. 2, p. 28.

1869. Larva, pupa. Harris, Lintom. Corres., p. 282.

187.. Larva, pupa, (col'd lig.) Boisdueal. Spece (iener. Splingidla, p. 5I, pl.1.

187t. Larvi, (1,rief.) R. Bunker. Canacl. Entom., vol. 8, 1. 120. 


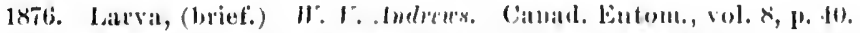

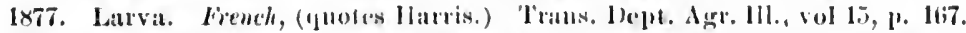

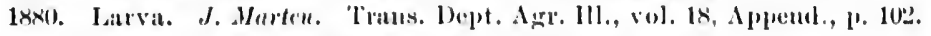

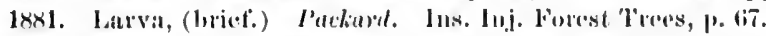

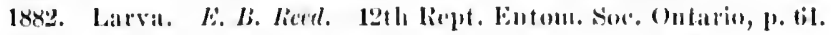

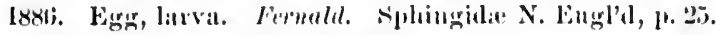

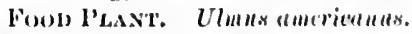

Daremma unduiosa. Nillk.

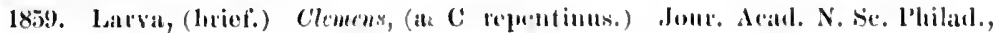
July.

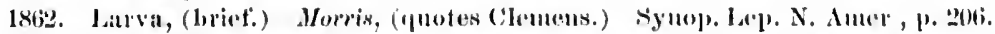

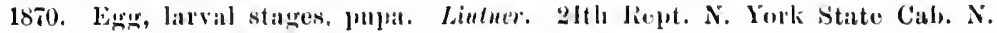
II ist., Pl. 12:3-131.

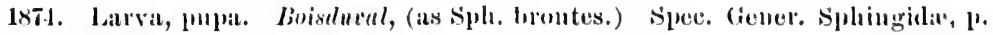
116.

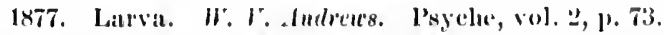

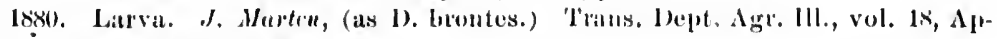
fend., 1. 102).

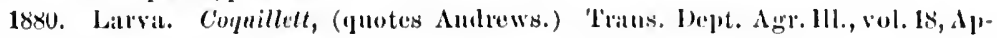
penil., p. 15!).

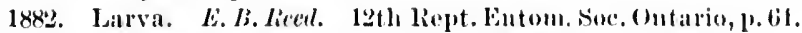

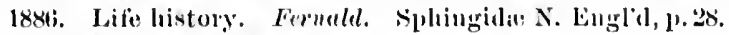

Foon Plant. Fraxinus.

Daremma catalpæ. Bois.

1874. Larva, finpa, (col'd tigs.) Loistlutal, (alter Abhot.) Spece (ienor. Sphingrida", p. 103, pl.:2.

1880. Lalval. J. Marlen, (quotes lioisduval.) 'Trams. Dept. Agr. Ill., vol. 18, $\Lambda_{1-}$ pent., p. 10.1.

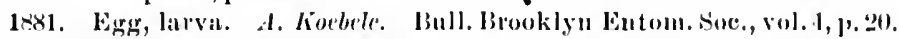

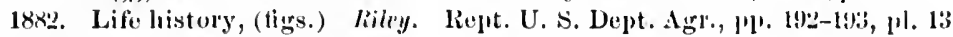

loon Plast. Cutalpa cordifolia.

Diludia jasminearum. Rois.-Lace.

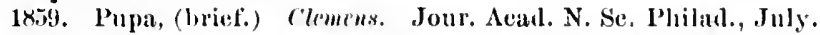

186.2. l'upit, (1) rief.) Morris, (anotes Clenens.) Syuop. Lep. N. Auter., p. 199.

187.1. Lat'va, pupa, (col'd tig.) Boistural. Spec. (iencr. Splinuridie, p. 115, pl. 1.

1880. Lallvi. I. Ifuten, ('plotes Boisduval.) 'Iraus. Dept. Agr. Ill., vol. 18, $\Lambda_{\text {p- }}$ pend., p. 103.

Food Plaxt. Fraximus.

Amphonyx antæus. Drury.

1719. Larva, fmpa, (eold ligs.) Mtedeme Merian. Ius. of Snrinau, pl. 2.

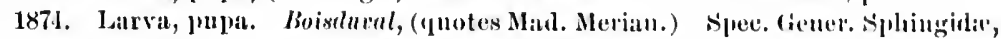
1. 65.

1886. Larvi, pupa. Gunlluth. Entom. Cubana, p. 206.

1887. Pupa. Hy. Eilwariz. Lutom. Amer., vol, 3, p. 11 it.

1888. Larva, (inief.) J. B. Smith. Monoy. Sphing., p. 15t

Foon Pl.axt. Species of Jutropha.

Phlegethontius rustica. Fal.

1797. Larva, pupa, (col'd tigs.) Abbot-Smith. Lep. Ins. Gicorgia, p. 67, pl. 3.1.

1858. Larva, (col'd fig.) Duncan. Natural. Library, vol. 22, p. 102, pl. (;.

1859. Larra, pupa. Clemens. Jonr. Acar. N. Se. I'hilad. (ฯuotes Mblot), July.

1862. Larva, pupa. Morris, (quotes Clemens.) Synop. Lep. N. Amer., p. 187.

1874. Larvi, pupa. loistural. Spec. Goner. Sphingidir, p. 83.

1878. Larva, pupa. M. Deritz. Wiegemaun Arehiv. Naturgesch., p. 10.

1886. Larva, pupa. Gundlach. Entom. Culana, p. 209.

1887. I'upil. Hy. Edwards. Eutom. Auer. vol. 3, p. 164.

Foon Prastrs. Chionththus, Jteminium. 
Phlegethontius carolina. L.

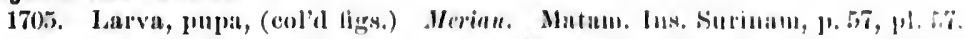

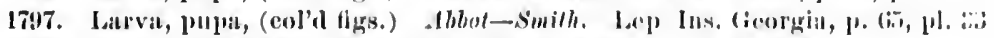

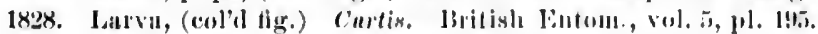

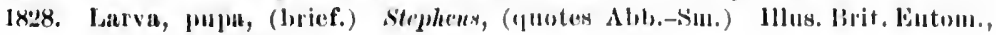
vol. 1, 1. 11x.

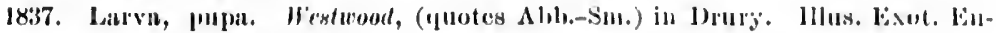
tom., vol. 1, 1. 47.

1834. Larva, pupa. Horris. Sillimun's Jour. Se. Art, vol. :il; p. 2:il.

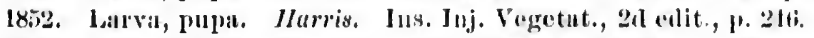

1859. Latri, pupa. Clemens. Jour. Acal. Sc, I'hilatl, July.

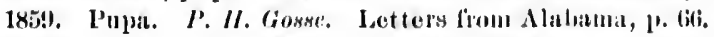

1862. Larva, I"lpa. Morris, (quites Clemens.) Synop, lap. N. Ames., p. 18!.

1869. Larva, puja, (figs.) Harris. Itus. Inj. Vegetat., Flint's rilit., 1. :i2:.

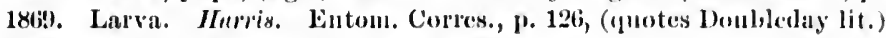

1869. Larva. Harris. Loc. eit., 1. 282.

1873. Larva, pupa, (figs.) T. Glerer. Reptt. U. S. Dept. Agre, 1. 177.

187.1. Larva. Boisalural, (after Ahbot.) Spec. (iener. Splhinginlid, p. it.

187.. Larva. Boistlutal, (val. lycopersiei.) Loc. cit., p. 72.

1877. Latrva. Freneh. 'Trans. Dept. Agr. Ill., vol. 15, p. 168.

1878. Larva, pupa. II. Dieriz. Wiegemann Arelıiv. Naturgeselı, p. 12.

1880. Larva. J. Murten. Trans. Dept. Agr, Ill., vol. 18, $\Lambda_{\text {pleend., }}$ p. 10:3.

1880. Larva. Coqnillett. Trans. Dept. Agr. Ill., vol. 18, $\Lambda$ plend., l. 158.

1882. Lalria. J. Leily. Proce A Aad. N. Se. Philad., p. 2:ia.

1885. Larva, pupa, (fig.) Feruald. Kingsley's Stund. N. Ilist., vol. 2, pl). l6il-1188.

1881;. Larva. Fornall. Sphingidie N. Engl'd, p. 32.

1886. Larva, pupa. Gundlach. Entom. Culani, 1. 210.

Foon Plants. Varions species of Solumum, Datura, ete.

Phlegethontius celeus. Hübn.

1811. Larva, pupa. Horris. Ins. Inj. Vegetat., Ist edit., p. 226.

1852. Larva, pupa, (brief.) Harris. Ins. Inj. Vegetat., 2 d edit., p. 217.

1859. Larva, pupa. Clemens. Jour. Acat. N. Sc. Philatl, July.

1859. Larva. Jacy'r. Life N. Amer. Ins., p. 189.

1869. Linva, pupa. Morris, (quotes Clemens.) Syuop. Lop. N. Amer., 1. 190.

1862. Larva, pupa, (figs.) Irurris. Ins. Inj. Vegetat., Flint's edit., p. 320.

1863. Larva, puga, (tigs.) Fitch. 9th Rept. Ins. N. York, p. 778.

186. Larva, pupa. Lintuer. Proc. Eutom. Soc. Phitad, vol. 3, p. (jls.

1867. Lalra, pupia, (tig.) S. Tenney. Manual N. IIistory, p. 392.

1869. Larva, pupa, (figs.) Hiley. 1st Missouri Rept., p. 95.

1870. Larva, (fig.) T. Glorer. Rept. U. S. Dept. Agr., p. 80.

1871. Larva, pripa, (figs.) E. B. Reed. Rept. Entwm. Suc. Ontario, 1. 119.

1873. Larva, pupa, (figs.) d. J. Cook. 12th Ropt. Stato Board Agr. Michigan, p. 117.

187. Larva. Joisduval, (after Abbot.) Spec. Gcner. Sphingidie, 1. 76.

1874. Larva. Gentry. Canad. Entom., vol. 6, p. 88.

1877. Larva. French. Traus. Dept. Agrr. Ill., vol. 15, p. 169.

1877. Latra, pupa, (fig.) I'ucliutrl. Inj. Ins. West, IIayden's Ropt., p. 780.

1877. Larva, pupa, (figs.) Packard. Ilalf-hours with lusects, p. 205.

1879. Larva, pupa, (figs.) Mirs. Ballarel. Insect Lives, .1. 95.

1880. Larva, pupa, (figs.) Bethune. Rept. Entom Soc. Ontario, p. 25.

1880. Larva, pupa, (figs.) Bethune. Canad. Entom., vol. 12, p. 101.

1880. Larva. J. Marten. Trans. Dept. $\Lambda \mathrm{gr}$. Ill, vol. 18, Append, p. 104.

1882. Larva, pupa, (figs.) E. B. Recd. 12th Rept. Entom. Soc. Ontario, p. 6ij.

1885. Larva. Lintner. Rept. Massachusette Board Agr., p. 180.

1886. Larva. Fernald. Spliugidle N. Engl'd, p. 34.

Foon Plas's. Solunum, Nieotiuna, Dutura, ete. 
Phlegethontius cingulata. Finlir.

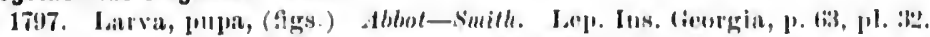

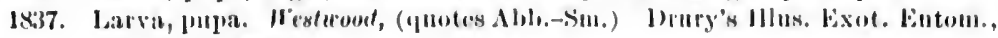
vol. 1, 1. 50.

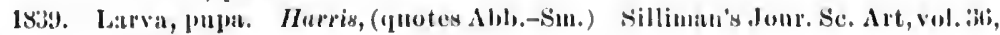
1.. 291.

1859. Larva, pupa. Clemens, (quoter Abh.-Sm.) Jour. Acnd. N. śe. l'hilad., July.

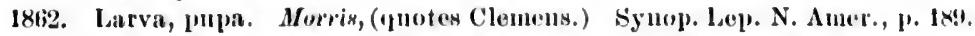

186it. Larva, pupa. Lintuer. I'roe, Entom, Soc. I'hilad., vol. 3, p. tist).

1869. Larva. Harrix, (as Spl. convolvoli.) Entom. Corres., 1. 28:.

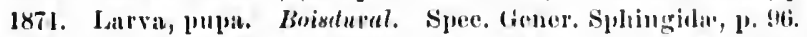

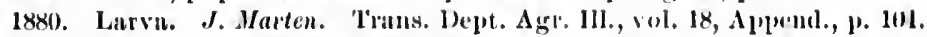

1880. Larva. Coquillett, (quotes Als,-Sm.) Trans. Dept. Agr. Ill., vol. $18, \Lambda_{1}$ pend., p. 158.

188t, Larra, pupa. Fermall. Sphingidion N. kngl'd, p. 36.

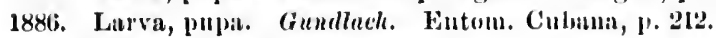

Foon Plane. Comroleulus bututas.

Sphinx drupiferarum. Alsb.-Su.

1707. Larva, pupa, (col'l tigs.) Abbot-Suth. Lep. Ins. (ieorgia, p. 7t, pl. 33 ;. 1839. Larva, pupa. Harris, ('potes Aht.-Sun.) sillimin's Jour. Sce. Art, vol. $36,1.294$.

18506. Larva. Fitch. 3il Rept. Ins. N. York, p. 362.

1859. Latra, pupal. Clemens. Jont. Aeat. N. Se. L'hilat, July.

186.. Larva, pupa. Morris, (quotes Clemens.) Synop. Lep. N. Amor., l. 197.

1864. Larva, pupa. Lintuer. P'roe. Entom. Soe. Plhiluel., vol. 3, p. 658.

1871. Ligg, larval stages, pupai, (figs.) E. B. Recd. Canal. Lintom., rol. 3, 1.5.

1871. Larva, pupa, (ligs.) E. B. Revel. Rept. Butom. Soc. Ontario, p. 36i2.

1874. Larva, pupa. Boisdural, (quotes Abb.-Sm.) Spec. Gener. Sphingide, p. 98.

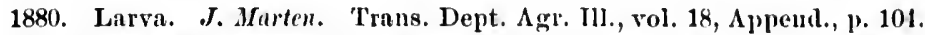

1882. Larva, pupa, (figs.) L. B. Reed. 12th Rept. Entom. Soc. Ontario, p.67.

1883. Larva, (brief.) Ph. Fischer. Canad. Entom., vol. 15, 1. 2:38.

1883. Egg, lavial stages, pupa, (figs.) Saurilers. Ins. Iuj. linuts, p. 1633.

1886. Egg, larva, pupa, (tigs.) Feruthl. Sphingidar N. Engl'd, je. 10.

Foon Plants. P'runus, Celtis.

Sphinx kalmix. Albl,-Sm.

1797. Larva, pupa, (col'd figs.) Abhot-Smill. Lep. Ins. Georgia, p. 73, pl. 37.

1839. Larva. IIurris, (quotes Abl.-Sm.) Sillinan's Jonr, Se. Art, vol. 36, 1. 295.

1810. Egg, young larra. I'. H. Gossc. Canarl. Naturalist, p. 260.

1859. Larva, pupa. Clemens, (yuotes Al,h,-Sm.) Jour. Acall. N. Sc. Mhiliul., July.

1862. Larva, pupa. Morris, (quotes Clemens.) Synop. Lep. N. Amer., p. 196.

1861. Larva, pupa. Liztuet. P'roc. Entom. Soc. Plhilat., vol. 3, p. 657.

1874. Larva, pupa. Boislural. Spec. Gener. Sphingiıliı, [1. !12.

1880. Larva. J. Marten. 'Trans. Dept. Agr. Ill., vol. 18, Aple'ull, 1'. 10.1.

1882. Larva. E. B. Recd. 12th Rept. Entom. Soo. Ontalio, p. 68.

1886. Larva, pupa. Fereuld. Sphingida N. Engl'd, p. 41.

Food Plants. Talute, Chionanthes, Syriega, Fraxinus.

Sphinx chersis. Hübu.

1864. Larva, pupa. Lintner. Proc. Entom. Soc. l'hilad., vol. 3, p. 6i55.

1869. Larva. IInrris. Fintom. Corres., p. 282.

(0i).

1877. Larva. Scudder. Psyche. vol. 2, p. 76.

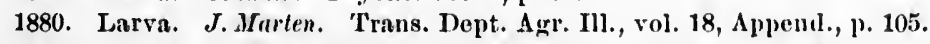

1882. Larva. E. B. Reed. 12th Rept. Entom. Soc. Ontario, p. 63.

1883. Larva. Edwardy-Elliot. P'inilio, vol. 3, p. 127. 


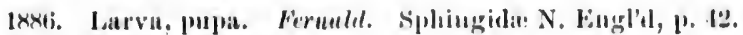

loul, P'lastr. syringu.

Spliinx gordius. Crmuser.

1X:3s. Larva. Harris. Silliman's Jour. Sc. Art, vol. 36, 1..295.

Ixisi. Larva. Fitch. 3it Rojet. Ins. N. York, 1. 3 II.

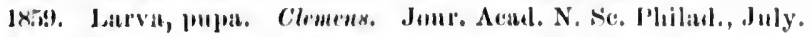

181;2. Larva, pupat. Murris. Symup. Lep. N. Amur., J. 1!k.

1877. Iatria. Patelitrel. IIall-liuurs with Insects, J. 180.

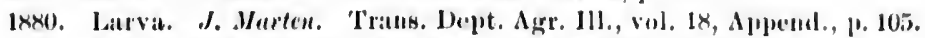

18x3. Larva, [u]m, (lorief.) Suauder. Ins. Inj. Fruits, I' Xij.

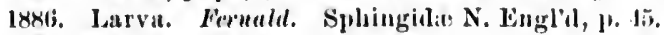

l'Uoll l'Lant, I'yinz.

Sphinx lugens. Walk.

1875 Larva, f'up. I'. H. Suow. 'Trans. Kiansas Acall. Sc., vol. I, J. 28.

Sphinx eremitus. Iliilın.

1870. Larva, (lig.) T. Glwere Jept. U.S. Hept. Agr., p. ki).

1879. Larva. T. W. Tylre. Camal. dintom., vol. 11, p. Ë.

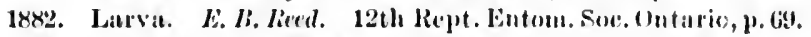

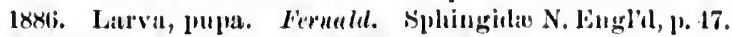

Foud Peasts. Mentha, Monerila, Sellia.

Sphinx elsa. Strecker.

1888. Larva. Hy. Edwards. Entom. Aner., vol. 1, 1. (iI.

Sphinx luscitiosa. Clem.

1888. Larval stages, pupa. Curoline (i. Sould, P'syclie, vol. 5. p. 85.

Dólba hylæus. Hrury.

1797. Larva, jupa, (col'd ligs.) Abbot--Smith. Lep. Ins. Geveria, p. b4, pl.35.

1837. Larva, pupu. I'atuood, (1notes Abl,.-Sm.) Hery. Illus. Exot. Entom., vol. 2,1 . 19.

1839. Larva. Hurvis. Silliman's Jour. Sc. Art, vol. 36, p. 29)(i.

1859. Larva, Impa. Clemens, (ฯuotes Abb.-Sun.) Jomr. Acad. N. Se. I'hilad., Jily.

1862. Larva, pupa. Morris, (gliotes Clemens.) Synop. Lep. N. Amer., p. 20..

187. Larva, pupa. Boistural. Spec. Gener. Sphingidar, l. 99.

1882. Larva. E. R. Reel. 12th Rept. Entom. Soc. Ontario, 1. 70.

1881. Larva. IV. II. IItrington. Canad. Entom., vol. 16, 1. 55.

1886. Larva. Fermald. Spllingielio N. Eugl'd, 1. 49.

Food Plant. Prinos.

Dilophonota ello. L.

1719. Larva, pupa, (col'd figs.) Madume Meriat. Iirs. Surinam.

1839. Jarva. Ilavis, (†uotes Mad. Merian.) Silliman's Jour. Sc. Art, vul, 36, 1.. 297.

1859. Larva, pupa. Cleme's, (џnotes Mad. Meriuu.) Jour. Aead. N. Sc. Philad.

18ti2. Larva, pupa. Morris, (quotes Clemens.) Synop. Lejr. N. Amer., 1. 201.

187.. Larva. Boisdural, (niter Merian.) Spec. Gener. Splingidae, p. 120.

1886. Egg, larva. IV. J. IJlland. Canad. Entom., vol. 18, 1. 103.

1886. Larva, pupa. Guullach. Enton. Cubana, p. 223.

1887. Larval stages, pupa. Hy. Edwards. Entom. Amer., vol. 3, p. 166.

Food Plant. Pridium.

Anceryx edwardsii. I3utler.

1887. Larval stages, pupa. Hy. Edwarls. Entom. Anei., vol. 3, p. 165.

Hyloicus plebeius. Fabr.

1874. Larva, (col'il lig.) pupa. Boisducal, (after Alıbot's drawing.) Speo. Gener. Splingidar, p. 100, pl. 1.

1877. Larva. Scudder. Psyche, vol. 2, 1. 78.

1886. Larva. Fernald. Sphingida N. Engl'd, p. 53.

Foon Plants. Bignonia, Passiflora. 
Hyloicus cupressi. Bois.

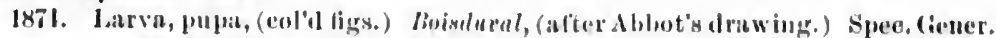
Splingridlin, p. 102, pl. 2.

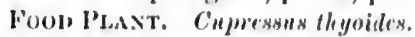

Ellema coniferarum. A!h,-Sin.

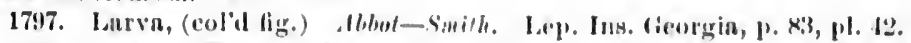

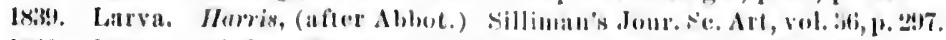

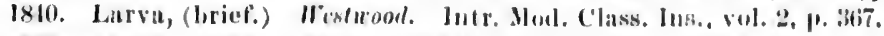

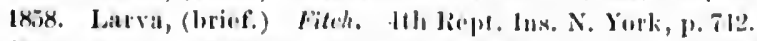

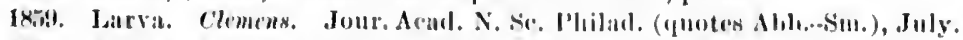

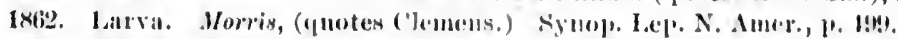

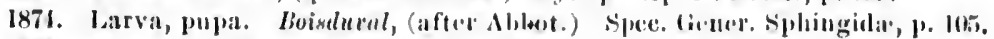

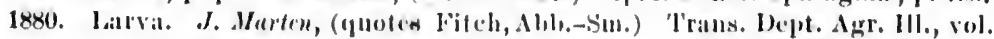
$1 \times, A_{\text {plpeul, }}$,. $10 \%$.

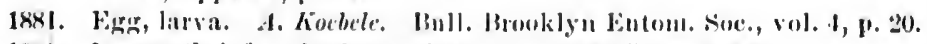

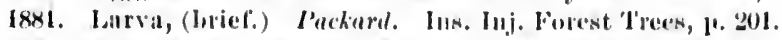

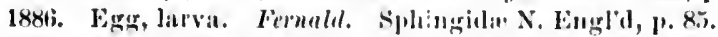

1887. P'upa. Hy. Villurarls. Entom. Amer., vol. 3, p. 1 ti7.

Foon Plast. Pinnes pulustrix.

Ellema bombycoides. Walk.

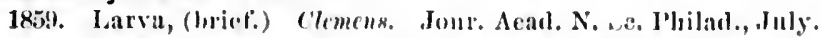

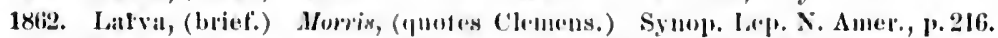

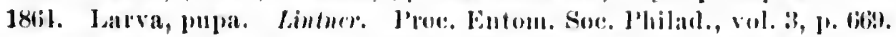

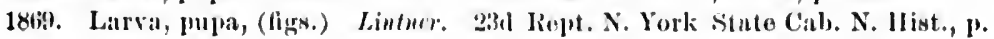
171.

1869. Latru. I'uckurd. Guide to Study of Jnsects, p. 272.

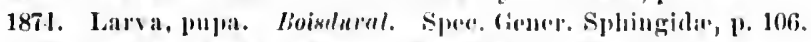

1877. Lalva, W. I. Ambers. lesyele, vol. 2, 1. 79.

1880. Larva. J. Marten. 'Trans. Dept. Agl' Jll., vol. 18, Append., p. 105.

1881. Larva, pupa. Packerel, (1)otres Lintucer.) lus. Inj. Forest Trees, p. 202.

1882. Larva. E. B. Rete. 12th lippt. Entonn. Soce. Ontario, p. 70.

1886. Larva. Fermalel. Splingidlir N. Eingl'l, p. 81.

Foon l'last', Pinus of varions npecies.

Ellema pineum. Lintu.

1869. Larva. Lintuer. 234 Rept. N. York State Cal. N. Ilist., p. 170.

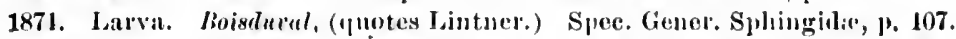

1880. Lalrva. J. Marten, (quotes Lintuer.) Trans. Dept. Agrr. Ill., vol. 18, Alpend., p. 10:.

1881. Larva. I'achard, ('unotes Lintuer.) Ins. lnj. Forest Trees, p. 202.

Foov Plaxt. I'inus atrobus.

\section{EGERIADE.}

Melittia ceto. West $w=$ cucurbitæ. Ilarr.

1828. Larva. Marris. N. Engl'd l'aner, vol. 7, p. 33.

1839. Larva, cocoon, pupa. Harvis, (as Aeg. cucurbite.) Silliman's Jour. Sc. dit, vol. 36 .

1812. Larva. Harris. N. Engl'd Furmer, vol. 20, p. 260.

1852. I'ula, coeoon, (brief.) IIarris. Ing. Inj. Vegetat., 2 l edit., p. 252.

1862. Larva, cocoon, pupa. Morris, ('fnotes Harris.) Synop. Lep. N. Amor., 1'. 139.

1862. Larva, cocoon, (figs.) Harris. Ins. Inj. Vegetat., Flint's edit., p. 331.

1819. Larva. ITarris. Lintom. Corres., p. 281.

1871. Larva, cocoon, (figs ) F. li. liect. Rept. Entom. Soc. Ontario, p. 429.

1874. Larva. Roisdural, (after Abbot.) Spec. Gener. Sphingida, p. 470.

1877. Larva, (fig.) Packard. Inj. Ins. IVest, Hayden's Rept., p. 769.

1877. Larva. French. Trans. Dejt. Agr. Ill, vol. 15, p.173.

BUI.L. 35-4 


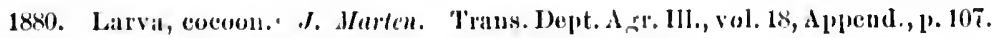

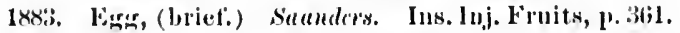

18\%:3. Larvi, pupri, (tigs.) Saunders. Loe. cit., p. 361.

1885. Lalvia, cocosn, (tigs.) Lintuer, (quotes llarris.) 2d Rept. Slate lintent. N. York, 1. 5\%.

188.T. Lalrva, pupa. Scudder. Psyehe, vol. 4 , p. 303.

Foon, ['tuxis. C'acurbilacte of various species.

Alcathoe caudatum. Harris.

1834. Larva, (brief.) Harris. Silliman's Jour. Sc. Art, vol. 36 .

18ti2. Larvi, (l,rief.) Monris, (quotes IIarris.) Synop. Lep. N. Amer., p. 139.

185ti. Larrat. Fitch. Bd liept. Ins. N. York, p. 421.

1877. Larra. French. Trans. Dept. Agr. Ill., vol.15, p. 172.

1880. Lalrvil. J. Arate'u. Tranis, Dept. Agr. Ill., vol. 18, Append., p. 103.

Foon l'Laxts. libes, Clematis, (stems.)

Trochilium apiforme. I.

1828. Larva, pupa. Stephecus. Illus. Brit. Eutom., Ilanst., vol. 1, 1, $1: 37$.

18:3i. Larva. J. Duacan. Natural. Library, vol. 4, Entom., p. 173.

1810. Larvi, pupia, (col'd tigs.) Ralzcbary. Die Forst-lnsecten, vol, 2, p. io, pl. :3, 4 .

1818. Larvi, (colil fig.) Hamplereys. Genera British Moths, p. 13, pl. i.

18.7. Larva. Sfaintou. Manual Brit. Butt. and Mothe, p. 102.

1872. Larva, (brief.) J. G. Iood. Insects at llone, p. 427 .

1872. Larvia. Figuier. Inseet World (Dunean's edit.), p. 193.

1874. Larva, cocoon, pupa, (figs.) I'. M. Inucan. Trunstor. of hos., p. 95.

1882. Larvi. W. l'. Kirlby. Europ. Butt. and Moths, p. 77.

Foon Praxiss. Trunks of Irillow and P'oplar.

Fatua denurlata. ILarris.

1852. I'upa, (briel.) IIarris. Ins. Inj. Vogetat., 2il eslit., p. 252.

1×62. P'npa, (brief.) Herris. Ins. Inj. Vegetat., Flint's edit., p. 330.

Foon Plast. Froxiuus, (stems and trunk.)

Bembecia marginata. Harris.

1874. Larva. Riley, (as Aig. lubi.) 6th Missouri Rept., p. 113.

1877. Jar, a. Freuch. Trans. Dept. Agr. 111., vol. 15, p. 175.

1880. Larva. J. Muten. Trans. Dept. Agrr. Ill, p. 18, Appenel., p. 108.

1881. Larva. Riley. Suppl. Missouri Ropt's, p. 72.

1883. Ligg. G. D. ITulst. Bull. Brooklyn Entom. Soe., vol. 6, p. 8.

loov Plaxt. Stems of Rubus.

Sciapteron tricincta. Harris.

1881. Larvi, (brief.) D. S. Fellicoll. Bull. Buffilo Soc. N. Sc., p. 62.

1881. Larvi, pupa. D. S. Fellicotl. Canad. Entom., vol. 13, 1. 3.

1881. Larva. Puckard, (quotes Kellicott.) Ins. Inj. Forest Trees, p. 121.

Foon Plaxt. Populus candicans, (stems.)

Sciapteron polistiformis. JIarris.

1851. Lawa, cocoon, pupal, (bricf.) Harris. Rept. on Ins. Inj. Vegetat. 'Irans, Amer. Pomologieal Soc., p. 10.

1856. Larva. Fitck. 3i Rept. Ins. N. York, p. 387.

1861. Larva, cocoon, (figs.) T. (ilorer. Rept. N. York State Board Agr., p. 72.

1869. Larval stagres, pupa. B. D. Walsh. 1st Illinois Rept., p. 24.

1871. Larva. Riley. 3il Missonti Rept., p. 76.

1877. Larva. French. 'Trans. Dept. Agr. Ill., vol. 15, p. $17 \mathrm{l}$.

1880. Larva. J. Horlen. 'Trans. Dept. Agr. Ill, vol. 18, Append., p. 108.

1883. Larva, coeoon, (figs.) Saunders. Ins. Inj. Fruits, p. 229.

Foon Puant. Tilis, (stem aud roots.)

Sciapteron anthracipennis. Boiscl.

1880. Larva. J. Murleu. Trans. Dept. Agr. Ill., vol. 18, Append., p. 109.

Food Plaxt, Salix, (stems.) 
Ha.jmonia pini. Lellicott.

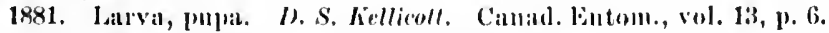

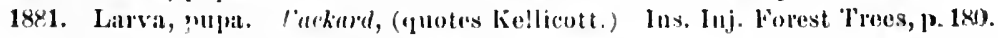
Food Plaxi, l'inus, (trunk iund stem.)

Podosesia syringa. Llarris.

1839. Latra. Harris. Sillinatu's Jonr. Sce. Art, rol. 36.

1871. Larra. Inckurd. Inj. Lns, new or little knewn, p. 18.

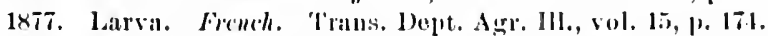

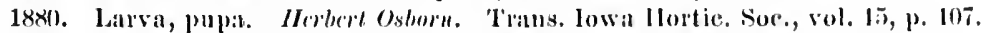

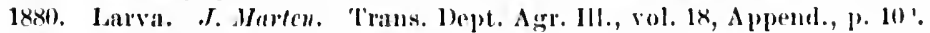

1881. Latrva, My. Eilnords. I'apilio, vol. 1, p, 181.

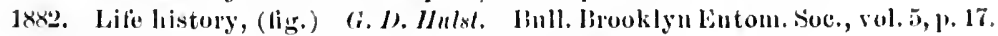
Food Plaxta. Fruximus, syringn, (trunk amd stem.)

Sannina exitiosa. Say.

182:5. P'upa, cocom. Say. Amer. Entom., vol, 2, pl, 19.

182ti. Lalra. Harvis. N. Engl's Farmer, vol. 5, p. 33.

1839. Latras. Harvis. Silliman's Jomr. Se. Art, rol. 36.

18.13. Eugr, latra. Willis Gaylorl. T'rans. N. York Agr. Soc., p. 161.

18.51. Latra, cocoon. Emmols. Nat. llist. N. York, vol. 5, p. 2e:3.

18.i. Legr, larva, pupa. Fitch, 1st Rept. Ins. N. York, pp. 108-11i.

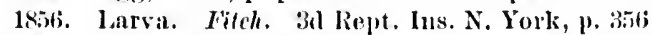

1859. Larva, pupa. P. H. Gosse, Letters from Alabama, p. 116.

186:. Larva. Morris, (qnotes Harris.) Synop. Lnp. N. Aner., p. 140.

18ti2. Larva, pupa, (tigs.) Harvis. Ins. Inj. Vegetat, Flint's edit., p. 331.

18ii. Larva. D. D. Wulsh. P'actical Entom., p. 6ī.

1869. Larva, Rile'y. 1st Mlssouri Rept., p. 47.

18i1. Larva. Sanuelers. Cimat. Entom., vol. 3, p. 22.

1xi3. Larva, puph. A. J. Cook. 12th Rept. Nichigan Board Agr., p. 136.

1876. Ligr, larva. Cyrne Thomas. 'Trans. Dept. Agr. 111., vol. 14, p. 38.

1877. Larva. Foneh. Trans. 1)pt. Agr. 11l., vol. 15, p. 179.

1879. Eigr. J, II. Comstoch. Rept. C.S. Dept. Agr., p. 254.

1880. Larva. I. Harten. 'Trans. Dept. Agr. I11, vol, 18, Appent., p. 107.

1883. Eugr, larr.u, pupa, (fig.) Sounders. Ins, luj. Frnits, p. 192.

1881. Eng, lawa, cocoon, (tig.) T. J. Lilge. Rept. Agr, Pennsylv., p. 101.

1885. Wigr, larvia. Fernull. Kingsley's Stand. N. Hist., vol. 2, p. 461.

Foon Plast, rersich, (peach trees.)

Egeria pictipes. G. and $R$.

1879. Life history, (tigs.) I. S. Builry. N. Anter. Entom., vol, 1, p. 17.

1880. Larva, pupa, (fig.) J. J/arten. Trans. Dept. Agr. Ill, vol. 18, Append., p. 109.

Foon Plast. P'rumix, (trmuk ind stem.)

Egeria pyri. Harris.

1839, Larva. Harris. Silliman's Jour. Sc. $\Lambda^{\circ+t}$, vol. 36.

1856. Larva. Fitch 3d Rept. Ins, N. York, 319.

1862. Larva. Harris, Ins. In.j. Vegetat., fil' at's elit., p. 335.

1862. Larva, (bricf.) Morris, ('pnotes Harris.) Synop. Lep. N. Amer, p. 111.

1877. Larva. Fench. Trans. Dept. Agr. Mll, vol. 15, p. 170.

1880. Larva. J. Mforten. 'Trans. Dept. $\Lambda \mathrm{gr}$. Ill., vol. 18, Append., 1. 107.

Foon Plaxt. Pyrus, (trunk and stem.)

Egeria acerni. Clenı.

1874. Larvi, cocoon, pupa, (figs.) Riley. 6th Missouri Rept., pp. 107-110.

1877. Larvil. French. Trans. Dept. Agr. Ill., vol. 15, p. 173.

1880. Larva, cocoon, (figs.) J. Marten, (after Riloy.) 'Trans. Dept. Agr. Ill., vol. 18. Appenil., p. 108.

1881. Larvia, (tig.) I'ackaril, (quotess Riley.) Ius. Inj. Fruits, p. 107.

1881. Lifo history. Saumler's. Canad. Entom., vol. 13, p. 69. 
1882. Life history, (brief.) Suluders, 12th Rept. Entom. Soc. Ontario, p. 20. Foov Planitr. aleer, (stems.)

Egeria tipuliformis. 1.

18*8. Larva. Stephens. Illus. Brit. Entom., Ilanst., vol, 1, 1, 112.

1839. Latra. Itarris, Silliman's Jomr. sce. Art, vol. 36.

18.18. Larva, (col'il fig.) IInmphreys. (iener. Brit. Moths, p. 13, pl. 4.

1851. Larva. IIarris. Ilovoy's Mlagil\%. Hortic, vol. 17, ]. Ql1.

1856. Latra. Fïlt. Bal Rept. Ins. N. York, p. 423.

16ir. Larva. Stainton. Mamual Brit. Hintt. and Moths, p. 106.

1862. Lalva. Hamis. Ins. luj. Vegetat., Flint's exlit., p. 331.

1865. Larva. 13. 1). Wals, Practical Entom., p. 29.

18і1. Life history, (brief.) Saunders. liept. Eutom. Soc. Ontario, p. 381.

1872. Larva, (brief.) J. G. Woot. Insects at Home, p. 427.

1873. Larva, cocoon. A. J. Cook. 12th Ropt. Boarl A rre. Mlichigan, p. 140.

1879. Eurge, larvat. Sanuders. Rept. Entom. Soe. Ontario, 1. 76.

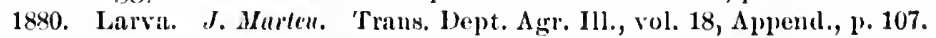

1880. Larva. Coquilletl. Truns. Dept. Agrr. Ill., vol. 18, Append., p. 151.

1882. Larva, (col'd fig.) W. F. Kirly. Europ. Butt. and Moths, p. 78, pl. 20

1883. Life history, (brief.) Selumelery. Ins. Inj. liruits, p. 336.

188;. Life history, (figs.) Lintuer. 20l lept. State Entom. N. York, 1) 60.

1888. Latril, puph, (figs.) Bethuc. 18th Rept. Entom. Soc. Ontario, p. 34.

Foon Prant. Ribes, (stems.)

Egeria prosopis. IIy. Hdw.

1882. Giall. Hy. Wdwards. I'ilpilio, vol. 2, p. 99.

FEEDis in stems of I'rosopis, making a gall.

Egeria hemizoniæ. Hy. Eaw.

1883. Larva. J. J. Rircers. I'npilio, vol. 3, 1. 26.

Foon Plast. linbur, (root and stems.)

\section{THYRIDA.}

Thyris vitrina. Boist.

1829. Larra. Boisdued, (after Abbot's drawing.) Monogr. Zygienida, p. 19.

187.. Larva. Boisducul, (after Abbot's drawing.) Spec. (iener, Sphingidir, p. 490.

Food Plant. Ihlascolas.

NoTE - This insect appears to he mnknown to American entomologists.

Alypia octomaculata. Fubr.

\section{ZYGENIDA.}

1797. Larva, (col'd fig.) Abbot-Swith. Lep. Ins. Georgia, 1. 87, pl. 44.

1839. Latrva. Harris. Silliman's Jour. Se. Art, vol. 36, p.314.

1854. Larvin, (col'd fig.) Emmons, (after Abb.-Sm.) Nat. IIist. N. York, vol. 5, pl. 38.

1854. Larva. Harrix. Rept. Ins, Inj. Vegetat. 'Trans. Amer. Ponological Soc., 1. 10.

1856. Larva. Fitch. 3l Rept. Ins. N. York, p. 399.

1862. Larva. Morris. Synop. Lep. N. Amer, p. 132.

1865. Pupa. l'ackarl. Notes on Zyganidir, Proc. Essex Inst., vol. 4, p.24.

1868. Larva. $W$. $I$. Andrews. Amer. Naturalist, vol. 2, p. 666.

1869. Lava, (fig.) Riley. 1st Missonri Rept., p. 136, pl. 1.

1869. Larva. Ilarris. Entom. Corres., p. 285.

1870. Lifo history, (tigs.) Riley. 2d Missouri Rept., p. 80, fig. 55.

1872. Larva. Linluet, (quotes Iarris.) 26th Rept. N. York Stato Cab. N. list., p. 120.

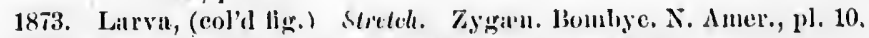

187.1. Larva, (fig.) likl'y. Go Missouri Rept., p. 9.1. 


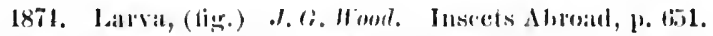

1877. Larra. Forneh. 'T'ras. Vept. Agr. 111., vol. 15, lo. 176.

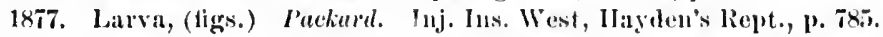

1878. Larva, (tig.) (i. M. Perlins. ittl Rept. Vermont lionar.l Agr., p. 276.

1880. Larva, (fig.) J. Jarten. T'rass. Dept. Agr. Ill., vol. 18, Append., l. 110.

1880. Larva, (fig.) ('oquillett, (yuotes hiley.) T'rans. Dept. Agr. Ill., vel. 18,

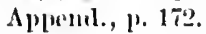

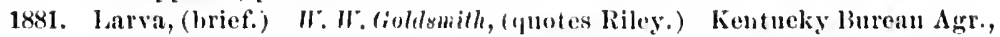
1. 2 is.

1883. Larva, pupa, (ligss.) Stumlers. Ius. Inj. P'ruits, p. 20(33.

1885. I,arva. Fenuld. Fingsley's Stand. N. Hist., vol. 2, 1. 463.

loon l's.axts. Jitix, Ampelopisis.

Psychomorpha epimenis. I) lury.

1869. Larva, (bricf.) Houbledu, (quotes Abbot iu lit.) ILarris's Entom. Corres., p. 180.

1871. Larva, pupa, (figs.) Liley 3al Missomi liept., p.61.

1872. Larva, (fig.) Linlues. 26tli licpt. N. Fork State Cab. X. Ilist., p. 121.

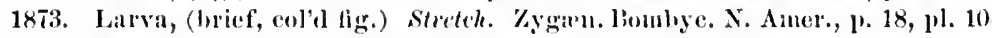

187t. Larva, pupa, (liers.) Lilly tith Missonri liept., p. x'.

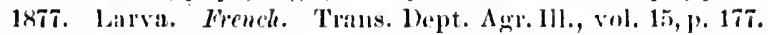

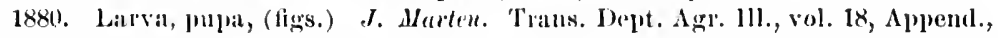
1). 111.

1.880). Jatra, (tig.) Coquillett, (quetes Lintuer.) Trans. Dept. Agr. 111., vol. I8, Alpeut., 1'. 172.

1883. Lavia, pupa, (ligs.) saunlers. Ins. Inj. Finits, p. 264.

loon I'tavers. Bigmonia ralirans, ritis.

Euscirrhopterus g'overi. (irota.

1887. Lifo history. L. A. Iopenoe. The Industrialist, Oet. 1, (quoted in lutom. Amer.), vol. 3, p. 178.

188x. Larva, pupa, (figs.) lílly. Insect Lilie, vol. 1, p. 104.

Hoon l'bast. Portulucu.

Eudryas unio. IIïm.

1856. Iarva. Iitch. 30 Rept. Ins. N. York. p. 399.

18isi. L'npa, (brief.) J. Kirliputriek. Proc. lintom. Soc. Philal., p. 43.

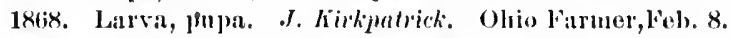

1870. Larva, (lig.) likley. 24l Missone hept., p. 83, (in errol for P. epimenis.)

1873. Larva, (hrief.) stretch. Kygien. Bombye. N. Amer. p. 150.

187. Iarva. Lintuer. 26tl, Rept. N. York Ntate Cal. N. II ist., p. 118.

18.t. Larva. Rilry, (rnotes Linture) (ith Missonri Rept., 1. 92.

1877. Larva. Fench. 'Trans. Hept. Agrr. 111., vol. 15, p. 178.

1880. Larva. J. Marten. 'Trans. J)pt. Agrr. Ill., vol. 18, Append, p. 111.

1880. Lavia. Coquillet, (quotes Lintuer.) Traus. Dept. $\Lambda$ gr. Ill., vol. 18, Append., p. 173.

1881. Larva, (luref.) J. s. Kellicott: Bull. Buffalo Soe. N. Se., vol. 4, p. 29.

1883. Larvi, pupa. Sunnilers. Ins. Iuj. Fruits, p. 262.

Foon Pes.., ritis.

Eudryas grata. Fabr.

1811: Larva. IItris. Ins. Inj. Vegetat., 1st eclit., p. 310.

1852. Larva. Harris. Ins. Inj. Vegetat., 21 edit., p. 399.

1856. Larva. Fitch. 3l Rept. Ins. N. Yolk, p. 399.

1859. Lauva. Clemens. Jour. Aend. N. Se. Philad., July.

1862. Larva, (lìss.) Harris. Ins. Inj. Vegetat., Flint's elit., p. 427.

1862. Larva. Morris, (†notes Harris.) Synop. Lep. N. Amer., p. 216.

186.1. Larva, pupa, (ligs.) I'uckard. Notes on Zygatu. Proc. Fssex Inst, vol. 4, p. 27.

1869). Larva. Harris. Hutom. Corres., p. 306. 
1869. Larva. I'ackired. Gintale to stmely of Insects, p. 281.

1×70. Larra, pinpa, (ligns) licley. 2d Missouri Rept., p. 83.

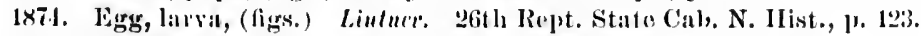

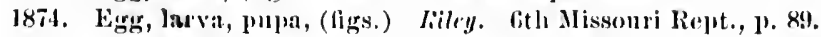

1875. Lito history, (figs.) Stundere. Camad. Entom., vol. 7, 11. 41.

187i. Eugr, larva, pupas, (figrs.) Sunders. Rept. Fintom. Soc. Ontario, p. :s.

1877. Lavva. Frenell. Trans. Dept. Agre. Ill., vol, 15, p. 17 \%.

1880. Eugg, larva, (figs.) .J. Murteu. 'Trans. Dept. Agr. 111., vol. 18, Append., p. 111.

1880. Lgg, larva, (figs.) Coquillett. 'Trans. Dept. Agrr. Ill., vol. 18, Append., p. 173.

1883. Ligg, larva, pupa, (ligs.) Suuuders. Ins. Inj. Frnits, p. 25\%.

1885. Egrg, larva. Fonald. Kingsley's Stand. N. Ilist., vol. 2, 11. 162.

1888. Lgg, larva, (ligs ) litetele't. Rept. Lintom. Dept. Agr. Canad., p. 30.

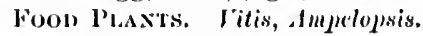

Cosmosoma omphale. Ilülı.

1886. Larvi, cocom. Gundluch. Entom. ('uhana, 1. 296.

1886. L'upa, cocoon. J. L. Swith. Eustom. Amer., vol. 1, 11. 186.

Foon Peant. Mikania.

Scepsis edwardsii. (irole.

1887. Larva. Hy. Edwords. Lintorn. Amer., vol. 3, ]. 167.

Scepsis fulvicollis. Iliilm.

1880. Larva. Coquillett. Canal. Entom., vol. 12, ?. 44.

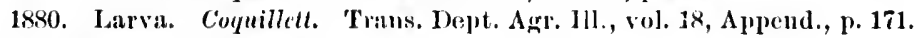

Foop P'tants. Grasses.

Ctenucha virginica. Charp.

1862. Larva, cocoon, (brief.) I'ackard. Tth Rept. Maino Buarl Agre, p. 168.

1864. F.gg, larvi, cocoon, pupa, (figs.) P'ackitrd. Notes on Kyginn. Proe. Essex Inst., vol. 4, p. 36.

1872. Larva, coeoon. Limtuer. EGth Rept. N. York Stato Calb. N. Ilist., p. 1.si.

1873. Larva, cocoon. Stretch, (quotes Packatil.) \%ygin'n. Bombyc. N. Amer., p. 26.

1877. Larva, pupa, (coldd ligs.) l'ackud. llalf-hours with lusects, (frontisp.)

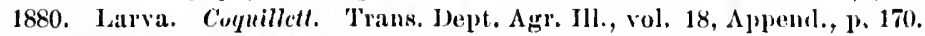

Foon Piavts. Grasses.

Ctenucha multifarin. Walk.

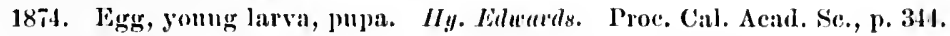

Acoloithus falsarius. Cleul.

1870. Larva, (lig.). T. Glocer. Rept. U. S. Dejt. Agr., 1. 81.

1880. Larva, (hrief.) J. Marten. 'Trans. Dept. Agr. Ill., vol. 18, $\Lambda$ ppend., p. 112.

Foon Plants. Titis, Ampelopsis.

Harrisina americana. IIarris.

1839. Larva. Hatris. Silliman's Jour. Sc. Att, vol, 36, 1. 315.

1844. Life history, (figs.) Ilaris. Hovey's Magazine, June vol. 10, p. 201.

1852. Larva. Harris. Ins. Inj. Vegetat., 2 d edit., p. 257.

1850. Larvit. Fitch. 3d liept. Ins. N. York, p.398.

1862. Larva, cocoon. IIarris. Ins. Inj. Vegetat, Flint's edit., p. 337.

1862. Larva, (hrief.) Packud. Rept. Maine Buard Agr., p. 168.

1870. Lifo history, (figs.) Riley. 2 il Missomri Rept., p. 85.

1873. Larva, (col'd fig.) Stretch. Zygaru. Bombye. N. Am r., pl. 10.

1877. Larva. Fench. Trass. Dept. Agr. 111., vol. 15, p. 179.

1880. Larva, cocoon. J. Marleu. 'Traus. Dept. Agr. 1li., vol. 18, Append., p. 112.

1881. Larra, pupa, (brief.) W. W. Goldsmih, (quotes Riley.) Kentucky Burean $\Lambda \mathrm{gr} .$, p. 255. 
1883. Eggr, larva, pupa, (ligs.) Sunuders. Ins. Inj. Frots, p. 20i\%.

1885. Larva, (tig.) Fernule. Kingsley's Stand. N. Ilist., vol. 2, 1. H(i2.

Foon P'lasess. Filix, Ampulopsis.

Lycomorpha pholus. Drury.

1839. Larva. IInmis. Silliman's Jonr. Se. Art, vol. 36, p. 318.

1862. Larva. Morris, (1notes Marris.) Synop. Lep. N. Amer., p. 135.

186\%. Iarva, (lorief.) IIaris. Ins. Inj. Vogetat., Fliuts entit.. p.311.

1873. Larva. Stretch, (quotes Intris.) Zyginn. Bomliye. N. Amur., l. I2.

1885. Larva. Fernald, (quotes Harris.) Kingsley's Staud. N. Ilist., vol.2, p. 162.

Foon Pisants. Lichens.

Oeta aurea. Fitch.

1869. Lavva, pupa, (figs.) Killy. 1st Missomri Rept., p. 15,l.

1873. Larva. Strelch, (quotes Riley.) Kygirn. Bombyc. X. Amre, p. 211.

1881. Eugre. Iill"y. Papilio, vol. 1, p. 110.

Fuod Plast. dilunthus.

\section{BOMBYCES.}

\section{Sub-fam. Nycteolidæ.}

Sarrothripa reveyana. S. V.

1859. Larva. H. T. Stuinlon. Brit. Butt.and Molls, vol. 2, p. 190.

18.9. Larva, cocoon, pupa, (col'd tigs.) Mumphrys. (ienera Brit. Motlis, p. 49.

1882. Larva. IF. F. Firby. Enrop. Bntt. and Moths, p. 2(l).

Food Plaxt, Sulic.

Nola ovilla. Grote.

\section{Sulb-funt. Lithosiidæ.}

1881. Larva, cocoun. Packurl. Amer. Naturalist, vol. 18, p. 720\%.

Fuon l'laxt. Quereus.

Nola sorghiella. Riley.

1882. Larva, pupa, (figs.) Riley. Rept. U.S. Dept. Agrr., p. 187, pl. 11.

Hypoprepia fucosa. IIillu., (iucl. var's.)

1841. Larva, pupa. Harris. Ins. In,j. Vegetat., 1st edit., [. 2.I1.

18:2. Larva. IItrris. Ins. Inj. Vegetat., 2d edit., 1..262.

1802. Larva, cocoon. Marris. Ins Inj. Vegretat., Flint's exlit., p. 312.

1862. Iniva. Morris, (quotes Ifarris.) Synop. I.pp. N. Aued, p. 25f;.

1871. Larva. Saumlers. Ganad. Entom., vol. 3, 1. 36.

1673. Larva, cocoon. Strelch, (quotes Ilarris.) Zyginn. Bomlyye. N. Amer., pt. 47.

1880. Lavia. Coquillet. 'Trass. Dopt. Agr. Ill, vol. 18, Appent., p. 181.

1880. Lavia. Coquilletl. Camad. Eutom., vol. 12, 1. Jis.

Foon Plant. Lichens.

Cisthene subjecta. Walk.

1881. Lajva, pupa. Mary E. Murlfeldt, (as C.packardi.) Psyche, vol. 3, p. 213. Foon Plant. Lirh'us.

Byssophagia nexa. Boisil.

1878. Egg, Jarva, pupa. My. Edwards. Proc. Cal. Acad. Sc., June.

Foos Plant. Lichens.

Eustixis pupula. IIülı.

1870. Larva, pupa, (fig.) Packarl, (after Abbot's drawing.) Amor. Nalurulist, vol. 4 , p. 229 .

Foon Plant. Silleronylum tenux.

Euphanessa mendica. Walk.

1871. Egg, larva. Saunders. Cauad. Entom., vol. 3, p. 227.

1873. Egg, larva. Streteh, (quoten Sammders.) Zygan. Bombye. N. Amer., 1, 53.

1886. Larva, pupa. G. D. IIulst. Hutom. Aner., vol. 1, p. itit. 
Phryganidea californica. I'ackard.

Sul-fini. Dioptida.

1873. Larva, pupa, (col'd ligs.) Stretch. Zygien. Bomlnye. N. Amer., p. 91, pl, 10.

187.. Egg, larval stakes. Hy. Lilurords. I'roe, Cal. Aeal. Se., Sept.

1877. Egg, larva. I'ackand, (unotes 11 y. Elwards.) luj. Ins. West, Hayden's Rept., 1. 808.

1881. Lige, larvil, pupit. Pation, (quotes Streteh and Iy. Edw.) Ins. Inj. Forest 'Trees, 1. 13.

Fool, Plant. Onerens.

\section{Sul,fan. Arctiidæ.}

Crocota quinaria. (isote.

1869. Larva. Sumders, (as Aret. bimaculata.) Canad. Lintom., vol. 2, p. 4.

Foon P'axts. Turarucum, Chenopolium, ats.

Utetheisa bella. 1 .

1870. Lallva, jupan, (ligs.) T. Glome. Rept. Dept. Agr., p. 80.

1880. Latra. J. Mart'n. 'T'ans. Dept. Agr. Ill, vol. 18, Append., p.113.

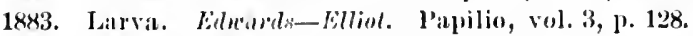

1886. Jarva, jupli. Ginullarh. Entom. Cubana, j. 258.

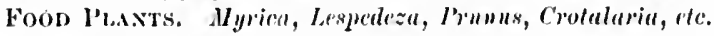

Callimorphá interrupto-märginata. De leauv,

1878. Larra, (brief:) C. G. Siewers. Cinat. Entom., vol. 10, p. 84.

Callimorpha coniusa. Lyman.

1887. Life history. H. II. Lymmtm. Cimal. Entom., vol, 19, p. 186.

Foou Plaxt. Cynoglosintm officinale.

Callimorpha clymene. Esp.

1871. Larva, (brief.) Riley. Bd Missonri Rept., p. 134.

Foon Plase. Querens.

Callimorpha lecontei. Boiscl.

1868. Larva. Sanule's. Canal. Entom., vol. 1, p. 20.

1871. Lalra, pupa, (ligss.) Le Baron. 2d Illinois Rept., p. 47.

1872 Larva. Limtmer. 26th Rept. N. York State Cal. N. JIist, p. 142.

1873. Larva. Stretch, (quotes Saunders.) Kygiru. Bombye. N. Amer., p. 6.1.

1877. Iarva. Fromel, ('1notes Samders,) Trans. lept. Agr. Ill., vol. 15, p. 181.

1880. Larva. J. Mforl('). Trans. Dept. Agr. Ill., vol. 18, Append., p. 115.

1883. Larva, pupa, (fig.) Samuler's. Ins. Inj. Fruits, p. 198.

Foon Plast. Triosteum perfolialmu.

Callimorpha fulvicosta. Clem.

1871. Isilva, pupia, (figs) Riliy. 3t Missouri Rept., p. 132.

1873. Larva, pupa. Strtch, (quotes Riley.) Zygien. Bombyc. N. Amer, p.66.

1877. Larva, (lig.) Fremcli, (after libley.) 'l'raus. Dejt. Agr. Ill., vol. 15, p. 180.

1880. Larva, (fig.) J. Iluten, (after Riley.) Trans. Dept. Agr. Ill., vol. 18, Appent., p. 113.

Foon Plants. I'yrus, Promus.

Epicallia virginalis. Boisel.

1873. Larrn, (brief.) P'aclime. IIayden's Survey Territ., p. 559.

1873. Larva, pnpa, (col'll tigs.) Streteh. Kygirn. Bombyc. N. Amer, p. 71, pl. 10.

Foon Prast. Lupiuns of vilrions speeies.

Platarctia parthenos. II:urris.

1868. Larva. Suunders. Cantul. Entom., vol. 1, p. 5.

1870. Egg, larval stages, cocoon. Linturr. 2/tlt Rept. N. York State Cab, N. Hist., p. 132.

1871. Larva. Saunders. Canad. Fntom, vol. 3, p. 225.

Foon Plants. Lacturet, ete.

Euprepla americana. Harris.

1863. Larva. Fitch. 9th Rept. Ins. N. York, p. 801.

1863. Larva. Saunders. Canad. .onjn., now series, vol. 8, p. 352. 


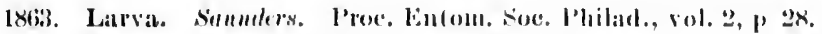

186t;. Larva. I'tekard. Proc. Boston soc. N. Ilist., vol. 11, 1. 31.

1869. Larva. H.tris. Litom. Corres., p. 287.

1870. Eger, larval stages, Limturr. 2Ith Rept. N. York State Cab. N. IIist., p. 131.

1873. Larva. Stretch. Zyganll. IBomber. N. Aurer., p. 9ti.

1875. Lifo history. li. Bunker. Camad. Futom, vol. 7, p. 119.

1876. Larva, (brief.) H. H. Lymu. Canal. Vintom., vol., , p. 20.

Foov Pissts. Lactuci, etc.

Arctia virgo. L.

1797. Larva, (col'd fig.) Abhot-Smith. Lep. Ins. (ieorgia, p. 12:3, pl.ti2.

1840. Larva, cocoon, (brief.) I'. $I$. Gosst. Canad. Naturalist, 1. 22y.

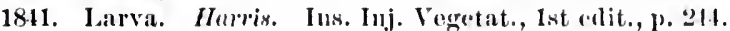

1852. Larva. Hurris. Ins. Inj. Vegetat., 2al edit, 1. 265.

1851. Larva. Eimmons. Nat. Ilist. N. York, vol. 5, p. 229.

1858. Larva, pupa, (col'd figs.) llumen. Natuml. Library, vol, 22, p. 17i;.

1862. Larva, (hrief.) Morris, (quotes Dnucan.) Synop. leep. N. Amer., p. 338.

1863. Larva, (bricf.) stunders. Camal. Jonlu, new series, vol. 8, p. 3is.

1864. Larva. Jeteger. Lifo N. Aner. Ins., p. 16 iti.

1873. Larva. Stretch. Zygirn. Homlye. N. Amer., p. 127.

Foon Plaxts. Chemporiucce, etc.

Arctia saundersii. (irote.

1863. Larva. Saumlers, (as A. parthenice.) Proc. Sintom. Soc. Philat., vol. 2, p. 28.

1863. Larva. Saunders, (as A. parthenice.) Canad. 'Tourn., new series, vol. \&, 1. 353.

1864. Larva. Grote, (quotes Samulers.) l'roc. Eutom. Soc. Plilad, vol. \$, 1. 75.

1873. Larva, pupar. Sanuder. liept. Entonu. Soc. Ontario, p. 23.

Food Plants. Chrnopodiuere', efe.

Arctia achaia. (i, and $R$.

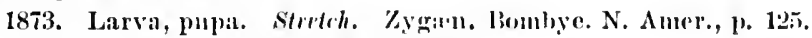

Foon Pinsts. Tifolium, Erodium, it

Arctia figurata. Imiry.

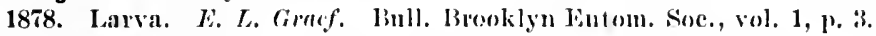

Arctia quenselii. Gryer.

1873. Larra, (tig.) I'teketrd. Hayolen's Surv. Territ., p. 5iss.

1882. Larva. W. F. Kirlb!. Europ. Butt. and Mothe, 1. 105.

Food Prants. Lonirera, Tarnacum.

Arctia phyllira. I)iny.

1797. Larva, (col'd lig.) Alhot-smilh. Lep. Ins. Georgia, l. 12т, pl. 61.

Arctia nais. Drury.

1877. Lava. French, (as A. phalerata.) 'Т'анs. Dept. Aerr. 1!1., vol. 15, 1. I82.

1880. Larva. J. Marten, (as $\Lambda$ phalerata.) 'Trans. lept. Agr. 1ll., vol. 18. Ap. pentl., p. 115.

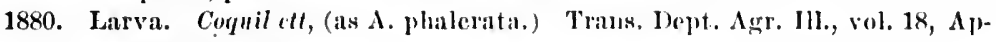
pend., p. 171.

1885. Fgg, larva. Frmald. Kingsley's Staml. N. Hist., vol. 2, p. 460.

Food Piants. Craminere, cte.

Arctia celia. Sannders.

1870. Larva. Sammdors. Canad. Entom., vol. 2, p. $7 . t$.

Arctia virguncula. Kirlyy.

1881. Larva. Coquillett. Papilio, vol. 1, p. 7.

Food Puant. Polygonum.

Arctia decorata. Sannders.

1881. Istra. French. Papilio, vol. 1, p. 81.

Arctia placentia. Abl.,-Su.

1797. Larva, (cul'd lig.) : lbhot-Smith. Lap. lns. Georgia, p. 129, pl. fis. 
Arctia arge. Drury.

1796. Larva, (col'd lig.) Abbot-Smith. Lep. Lus, (icorgia, p. 125, pl.63.

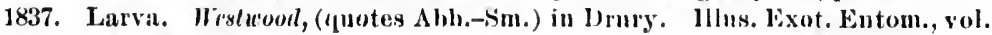
1, p. 3 .

1811. Larva. Harris. Ins. Inj. Vegetat., Ist edlit., p. 2.1.

1852. Larra. Marris. Ins. Inj. Vegetat., 2d edit., p. 2ti5.

18:4. Larva, pupa, (figs.) Limmons. Nat. IIist. N. York, vol. 5, pl. 41.

1858. Larva, (col'd fig.) Duneun. Natural. Library, vol. 22, 1, 175.

1862. Latrva. IItwris. Ins. Inj. Vegetat., Flint's edlit., p. 316.

1862. Larva. Morris, (quotes Duncan.) Synop. Lep. N. Amer., p. 340.

1863. Larva. Sannders. Canad. Jour., new series, vol. 8, p. 3in, (as $\Lambda$, dione.)

1864. Larva. Jaeger. Life N. Amer. Ins., p. 168.

1869. Larva. Harris. Lintom. Corres., p. 286.

1872. Larva. Lintner. 26th Rept. N. Y. State Cah. N. Ilist., p. 113.

1873. Larva. Streteh, (quotes Harris.) Zygann. Bombyc. N. Amer, p. 225.

1874. Lavva. Peaborly. Canal. Entom., vol. 6, p. 98.

1877. Larva. French. Trans. Dept. Agr. Ili., vol, 15, p. 182.

1877. Egg, larva. W: 1. Aulburs. Psyche, vol.2, p. 79.

1880. Larva. J. Murten. Trans. Dept. Agr. lll., vol. 18, $\Lambda$ ppend., p. 115.

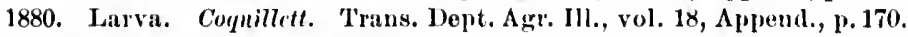

Food Plant. Anothera biennis.

Arctia brucei. IIy, Elwards.

1888. Larva. D. Bruce. Entom, Amer., vol. 3, p. 219.

Food Praxts. Polygonum, Plantayo.

Arctia remissa. IIy. Ellwards.

1888. Egg, yomug larva. Hy. Edwarls. Entom. Amer., vol. 3, p. 184.

Note. The food plants of the speeies of Aretia aro not always given, as the caterpillars are ahnost omnirorons, feeding npon any kind of herbaceons plants.

Kodiosoma nigra. Stretch.

1873. Larva, (brief.) Streteh. 'Zygian. Bombyc. N. Amor., p. 68.

Seirarctia echo. $\Lambda$ bb.-Sm.

1797. Larva, (colil figs.) Abbot-S'mith. Lep. Ins. Cieorgia, p. 185, pl. 68.

Seirarctia clio. Packard.

1882. Larva, cocoon. Behr. Papilio, vol. 2, 1. 187.

Foop Piant. Apocynum.

Pyrrharctia isabella. Albu.-Sin.

1797. Larva, (col'd fig.) Abbot-Smith. L.ep. Ins. Georgia, pl, 66.

1840. Larva. P. H. Gosse. Canad. Nuturalist, ply. 78, 308.

1811. Larva. Marris. Ins. Inj. Vegetat., 1st elit., p. 253.

1832. Larra. Harris. Ins. Inj. Vegetat., 2l elit., p. 273.

1851. Larva. Emmons. Nat. Jist. N. York, rol.5, p. 229.

1862. Larva, cocoon, (fig.) Irarris. Ins. Inj. Vegetat., Flint's edit., p. 355.

1863. Larva. Sannder's. Cauad. Jonr., new series, vol. 8, p. 36 .

1867. Larva. S. Tenney. Manual N. Iist., p. 397.

1872. Larva, pupa, (fig.) Riliy. 4th Missouri Rept., p. 143.

1873. Larva, cocoon, (figs.) Saunlers. Canal. Entoul, vol. 5, p. 76.

1873. Larva, cocoon, (figs.) Sannters. Rept. Futom. Soe. Ontario, p. 22.

1874. Larva, cocoon. $\Pi_{y}$. Eelwards. Proc. Cal. Aead. Se., September.

- 1874. Larra, (fig.) J. G. Wood. Insects Alhroad, p.664.

1877. Larva. Freneh. 'Trans. Dept. Agr. Ill., vol.15, p. 182.

1877. Larva, cocoon, pupa, (tig.) B. Golt. Rept. Lintom. Soo. Ontario, p.46.

1878. Larva. B. . Mann, (quotes Iarris.) Psycho, vol.2, p. 270.

1880. Larva, cocoon, pupa, (figs.) Riley. Amer. Entom., 2d sories, vol. 1, p. 133.

1880. Larva, cocoon, pupa, (figs.) J. Marten. 'Trans. Dept. Agr. Ill., vol. 18, Append., p. 115. 
1880. Larva, coeoon, pupa, (iirs.) Coqnillell. Low. cit., 169.

Foon Puavts. Varions low herhs.

Phragmatobia rubricosa. IIarris.

1810. Larva, (brief.) I’. $H$. (ioser. Canad. Naturalist, p. 195, (as P. fuliginosa.)

1883. Larvat, cocoon, pupa. Edurerds-Elliot. L'apilio, vol.3, p.127.

1887. Young larva. Hy. Ederards. Entom. Aner., vol.3, p. bis.

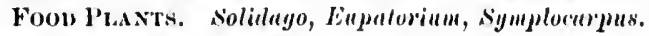

Antarctia vagans. Boistl.

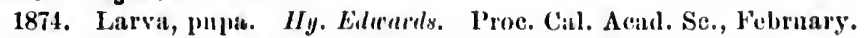

Foon Plast. Ptrits.

Antarctia punctata. Packard.

1873. Larva, cocoon. Strith. Zygan. Bomluyc. N. Amer., p. 194.

Food Plast. Tapiutus.

Leucarctia acræa. Drury.

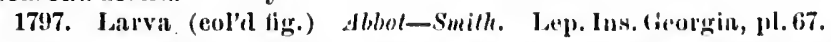

1823. Larva, pupa, (figs.) Harris. Matssaclunsett's Agre. Reposit., p. 322.

1841. Larva, pupa, (tigs.) Itaris. Ins. Inj. Vegretat., 1st edit., p.24!.

1852. Larva, pupa. Harris. Ins. Iuj. Vegetat., Sd edit., p. 26is.

1854. Larva. Emmons. Nat. Hist. N. York, vol. 5, p.226.

1858. Larva, pupa, (tig.) Duncun. Natural. Library, vol. 22, p. 172, pl. 20.

1862. Larra. Hurvis. Ins. Inj. Veretat., Flint's edit., p.351.

1862. Larva. Morris. Synep. Lep. N. Amer., p. 342.

1863. Larva. Stunders. Canad. Jour., new neries, vol. 8, p. 363.

1867. Larva, pupa, (ligs.) S. Tiune.y. Manual N. Ilist., p. 34t, pl. 29.1.

1874. Yonug larva. Hy. Eiluards. Proe. Cal. Acatl. Sc., Septemler.

1873. Larva, (eol'd fig's.) Sheleh. Ky g:rn. Bomlige., p. 101, pl. 10.

1877. Larva. C. Thomas. Trans. Dept. Agr. Ill., vol, 15, p. 79.

1877. Lasva. Freweh. Loc. eit., p. 183.

1879. Larva, pupa, (figs.) Mrs. Bullurl. Inseet Lives, p. 57.

1880. Larva. J. Afurte'n. 'Trans. Dept. $\Lambda$ gr. Jll., vol. $18, \Lambda$ ppend., p. 115.

1880. Larva. Coquillett. Traus. Dept. $\Lambda$ rrs. Ill., vol, $18, \Lambda_{\text {ppend., p. } 170 .}$

Foon Plavers, Varions low herhs.

Spiiosoma virginica. Faln.

18.1. Larva, pmpa. Ihuris. Ins. Inj. Vegetat., 1st edit., p. 2I8.

1852. Larva. Ifarris. Ins. Inj. Vegetat., 2d elit., p. 268.

1856. Larva. Fileh. 34 Rept. Ins. N. York, p. 100.

1862. Larva, (fig. I/arris. Ins. Inj. Vegetat., Flint's edit., p. 349.

1862. Larva. Morvis. Symop. Lep. N. Aner., p. 31 .

1863. Larva. Saundery. Camal. Jont., new series, vol. 8, p. 362.

186t. Larva. Jaeger. Life N. Amer. Ius., p. 168.

1869. Larva, cocoon, pupa, Harris. Entom. Corres., p. 287.

1871. Larva, pupa, (figs.) Riley. 31 Missouri liept., p. 68.

1871. Larva, pupa, (figs.) Sumulers. Rept. Futom. Soc. Ontario, p. 338.

1872. Larva. Lintner. 26th Rept. N. York Stnic Cab. N. Hist., 1. 143.

1877. Larva. C. Thomas. 'T'ans. Dept. $\Lambda$ gr. Ill., vol, 15, p. 83.

1877. Larva. Fremch. 'Trans. Dept. Agr. Ill., vol. 15, p. 183.

1878. Larva, pupa, (figs.) G. IT. 'Prikins. sth Rept. Vermont Board Agr., p. 273.

1879. Larva, coeoon, pupa, (tigs.) Mirs Bulluril. Inseet Lives, p. 5.t.

1880. Larva, pupa. Saunders. Cauad. Eintom., vol. 12, p. 56.

1880. Larva. J. Marten. Trans. Dept. Agrr. Ill., vol. 18, A ppend., p. 116.

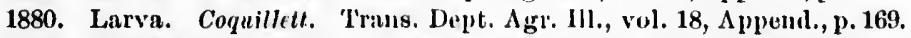

1880. Life history, (figs.) Saunders. Rept. Eutom. Soc. Ontario, p.21.

1831. Larva, pupa, (figs.) Pialiarl, (after Riley.) Ins. Inj. Forest 'Trees, p. 88.

1883. Life history, (figs.) Suumders. Ins. Iuj. Fruits, p. 273.

1887. Egg, larra. D. Biuce, (melunic var.) Eutom. Amer, vol. 3, p. 140.

Food Plants. Various. 
Spilosoma congrua. Wisllier.

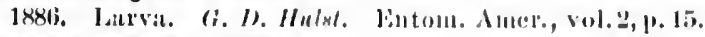

Spilosoma vestalis. P'uckitrl.

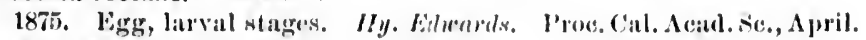

Fo(1) I'taste. Lupinus.

Spilosoma latipennis. Sitretr.h.

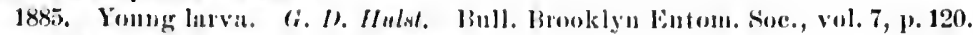

Hyphantria cunea. Hrury. (iuclucl. viır...)

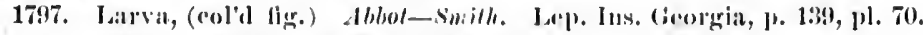

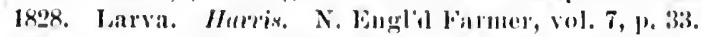

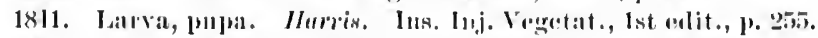

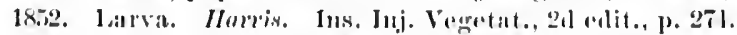

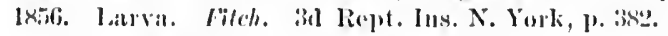

1869. Larvi, cocom, pupa, (ligs.) Iltivis. Ins. Inj. Vougetat., Flint's edit., 1. 3.58 .

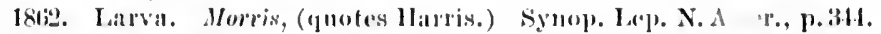

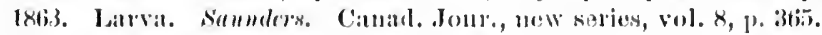

18is\%. Latrat. Harrix. Eutom. Corres., p. 360.

1870. Larva. Sammers. Cantal. lismome, vol. 3, p. 31;.

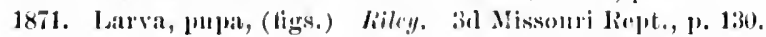

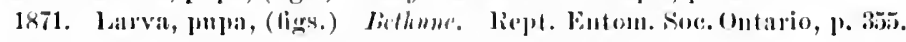

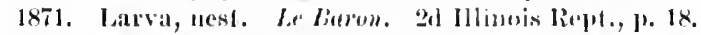

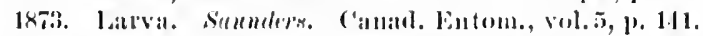

1873. Latra. Strdeh, (quotes Sammers-liiley.) \%ygrin. Bombye. N. Amer., p. 206.

1873. Larva, pnpa, (ligs.) Stumders. Rept. Eutoun. Soc. Ontario, p. 13.

1875. Larva, pupa, (firs.) A. I. Cook. 12th Rept. Michighu loard Agr., j. 152.

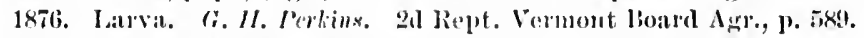

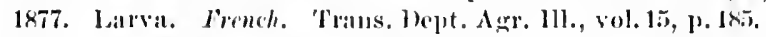

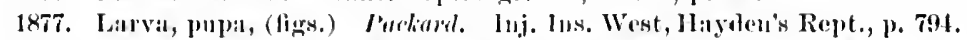

1879. Larva, pupas, (ligs.) II. Culling. Kepl. N. llampslige Board Agr., p. 22.

1880. J.arva, pupa, (figs.) J. M/urt'n. Thans. Dept. Agr. Ill., vol. $18, \Lambda_{\text {plpend., }}$ p. 111 .

1880. Larva, pupia, (ligs.) Coquilldt. Trons. Dept. Agr. Ill., vol. 18, Append., p. 15.1.

1881. Jatru, pupa, (figs.) P'adimm, (quotes Riley.) Ins. Inj. Forest Trees, p. 67.

1883. Lavra, (luriof.) X. colomen. P'ipilio, vol.3, p. 26.

1883. Larva, cocom, pupa, (ligs.) Sunndres. Lus. Inj. Frnits, p. 71.

1887. Lile listory, (figr.) Lill't. IBull. 10, Div. Eutum., U. S. Dept.Agr., pp. $30-i 8$.

1887. Lifo history, (tigs.) Rilly. Rept. L. S. Dept. Agr.,1886, pp. 518-539, pl. $10,11$.

1888. Larva, jupa, (fig.) Befhnne. 18th Rept. Futom. Soc. Ontario, p. 58.

Foon Plants. Varions trees and slimules.

Euchætes egle. Drory.

1811. Jarvil, pupit. ITarris. Ins. Inj. Vegetat., Ist edit., p. 256.

18:2. Larva, cocoon, pupa. ILaris. Ins. Inj. Veagetat., 2d edit., p. 276.

1862. Larva, cocoon, jmun, (figs.) Ilurris. Ins. Inj. Vegetat., Inlint's edit., p. 359.

1862. Larva, (brief') Morris. Symop. Lep. N. Amer., p. 313.

1863. Larva; pmpa, (1nref.) Stunters. Canal. Jonr., new series, vol. 8 ; p. 366.

1869. Larva, (figss.) Harvis. lintom. Corres., p. 288.

1870. Larva. Lintuer. 21 th Rept. N. York Stato Calı. N. Hist., p. 136.

1873. Larva. Stretch, (quotes Harris.) Zygan. Bomblye. N. Aner., p.186.

1878. Larva. W. I. Audeers. Psyche, vol. 2, p. 271.

1880. Life history, (brief.) H. S. Jewcll. Canal. Eıtom, vol. 12, p. 230. 


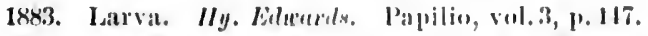

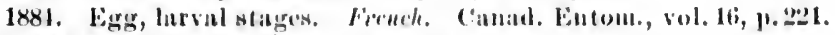

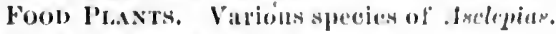

Euchates eglenensis. Cleurus.

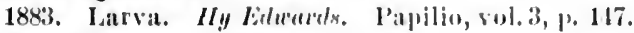

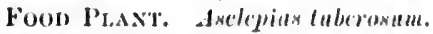

Euchates collaris. l'iteli.

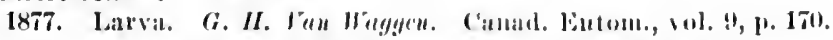

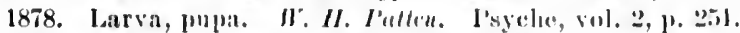

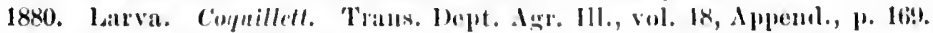

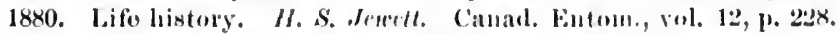

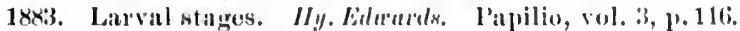

Foon l'sants. Apocyutum, isclepias.

Arachnis aulea. Loisil.

1883. Larva, jupa. W. Schuns, jr. I'apilio, vol.3, p. 188.

Foon, lisaxts. Varions low herbs.

Arachnis picta. Packard.

1873. Larvi, pupa. Streteh. Zygien. Hombye. N. Amer., 1.81, pl. 10.

Foon Plaxt. Latriutus.

Ecpantheria scribonia. Stoll.

1797. Lalva, (col'rl fig.) Abbol-Smilh. lap. Ius. (ieorria, J. 13i, pl. lis.

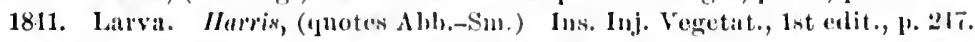

1858. Larva. Jutreu. Natmal. Libury, vol. 22, 1. 16i?.

1862. Latra. Molris, (4notes Dnncan.) Symop. Lep. N. Amer., p. 3.17.

1863. Lavia. Sanuders. Proc. Fintons. Soc. Plitad, vol. 2, p. 2x.

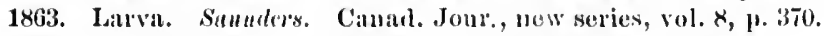

1872. Larva, pupa, (fig.) líly. Ath Nissouri Rept.,p. 141.

1877. Larva. Fiench. 'Trans. Dept. Agr. Ill., vol. 15, p. 181.

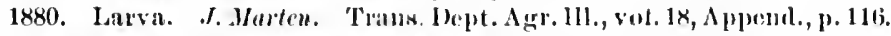

1882. Larva, (figr.) Sanuders. Camal. Eintom., vol. 11, p. 113.

1882. Larva, (fig.) Sanders. Rept. Entom. Soc. Ontario, p. 15.

Foon l'axirs. Melianthus, Illantayo, Salix.

Ecpantheria reducta. (ir.

1887. Larva. D. Bruce. Eutom. Amer., vol.3, p.1.1.

Foov Plaxts. Turaxatum, Strliuriu, etc.

Halisidota tesselata. Abh-Sun. (iucl. vars.)

1797. Larva, (col'd figg.) Ahhol-Smith. Icp. Ins. Georgria, p. 149, pl. 75.

1810. Larva, cocoon. I'. II. Gossc. C'aual. Naturalist, 1.2913.

1841. Larva, pupa. Iarris, (quotes $\Lambda$ bl,.-Sm.) Ius. Muj. Vegetat., 1st edit., 1.25̈).

1852. Larva. Matris. Ins. Inj. Vegetit., 2 ed edit., 1. 280.

1862. Larva. Morris, (quotes llaris.) Symop. Lep. N. Amel., 1. 3.9.

1862. Larva. Harris. Ins. Inj. Vegetat., Flint's edlit., 1).361.

1863. Larva. Saumlers. Canal. Jonr., new series, vol. 8, 1. 367.

1863. Larva. B. D. Walsh. Proc. Boston Soc. Lt. Ilist., vol.9, p. 289.

186.1. Larva. B. D. Walsh. Proc. Entom. Soc. Philad., vol. 3, 1. 413.

1864. Larva. B. 1). Falsh. Proc. Entom. Soc. Pliblat., vol. 3, 1. 430.

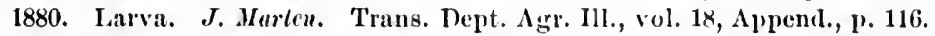

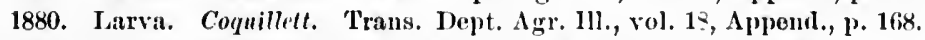

1885. Larva. Feruald. Kingsley's Stand. N. IFist., vol. 2, p. 460.

Food Piant. Acer.

Halisidota caryæ. Jairıs.

1811. Larva, pupa. Harris. Ins, nj. Vegotat., 1st edit., p. 258.

1852. Iarva, pupa. IItris. Ins. Inj. Vegetat., 2d edit., p. 27!).

1854. Larva, jupa, (figs.) Fiteh. 1st Rept. Ins. N. York, p. 160.

1856. Larva. Fitck. 3d Rept. Ins. N. Iork, p. 458. 


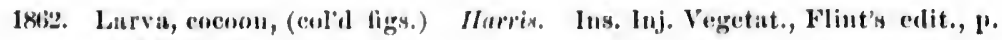
3iil, pl. (3.

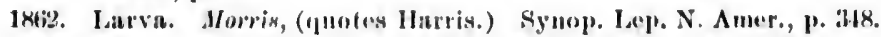

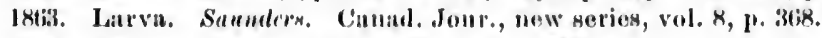

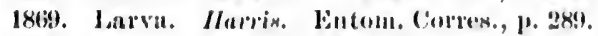

1872. Cocoon. Lintuer. etitl Rept. N. York Stuto Cab. N. Ilist., J. 1.1X.

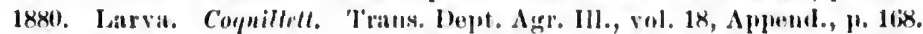

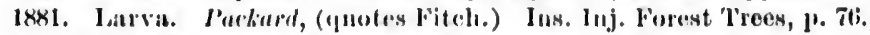

Foon l'zasts. C'arya, Jn!lume, cle.

Halisidota maculata. Ilarris, (incl. vars.)

1811. Lavia, g"1pat. Mamix. Ins. Inj. Vegotat., Ist colit., p. 2is!.

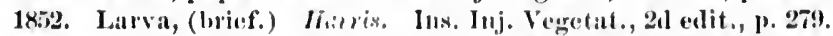

186:. Ianvi, (loriof.) Jlariv. Ius. Inj. Vougotnt., Flint's edlit., 1. 363.

1863. Larva. Saunder. C'anal. Jour., now series, vol. 8, p. 3tis.

1869. Jarva, (lig.) Jinrix. lintoun. Corres., p. 290, pl.9.

1869. Larva. I'ackird. (inide to Study of Ins., fl. 287.

1871. Latru. Sanuler. Canal. Jintoun., vol. 3, 1.186.

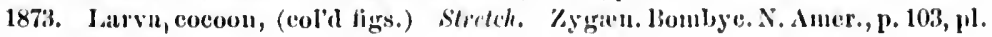
10.

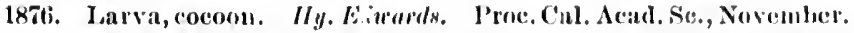

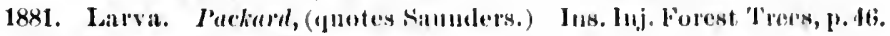

Foon Plants. Sulix, I'upulus, cle.

Halisidota argentata. I'ackarul.

1874. Jarva, cocoon, pupa. My. Falurals. I'ruc. Cal. Acad. Se, September.

Foon Pl.sxts. biune, dbir's.

Halisidota sobrina. Strefcl.

1874. Larvi, pupa. Hy. Edurarly. L'roc. Cal. Acad. Se., September.

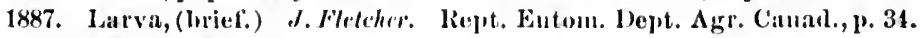

Foon Plast, Pinuk.

Halisidota edwardsii. P'ackard.

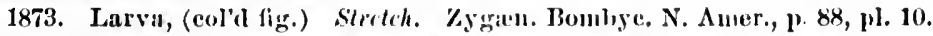

1875. Ege, young larva. IIy. Edwards. Proc. Cal. Acad. Sc., Ajril.

Fool) Prant. Quercus.

Halisidota cinctipes. Groto.

1886. Larva. Condlach. Enfoun. Ciulana, 1.269.

Fools Plaxt. Mibiscus.

Sub-fau. Pericopidæ.

Gnophæla hopfferi. (4. and li.

1882. Larva, (brief.) Strelch, (Juotes Lord Walsingluam iu lit.) Papilio, vol.2, 1. 82.

Foos Pisat. Myosolis.

Gnophæla verniculata. Gr. Rob.

1888. Larva. I). Bruce. Entom. Americana, vol. 4, p. 2 f.

1889. Larva. T. D. Cockerdl. Entum. Amer., vol. 5, 1. 57.

lood Plant. Martensia rirginicu.

Melanchroia geometroides. Walker.

1886. Iarva, pupa. Gunellach. Sintom. C'ubana, 1. 410.

Food Pinat. Circa racimosa.

Orgyia nova. Nitch.

Sub-funt. Liparidæ.

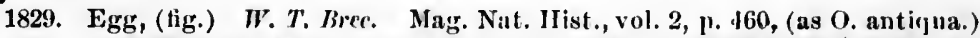

1841. Isarva, pupa. Hurrik, (ns O. antiøna.) Ins. Inj. Vegetat., Ist edit., p. 262.

1852. Larva, cocoon. Harris, (as O. antiqua.) Ins. Inj. Vequtat., 2lledit., p. 284.

1862. Life history. Fitch. 8th Rept, Ins, N. York, p.67i. 
1862. Larva, cocom. Murin, (as O. antiqua.)Ins. Luj. Vegetat., Flint's edit, 1.. $36 \%$.

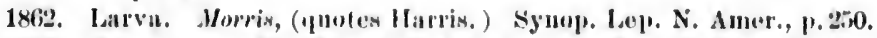

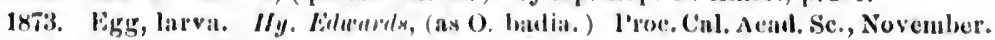

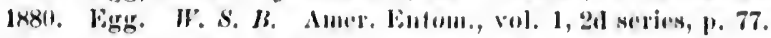

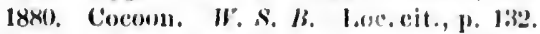

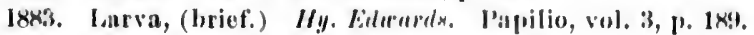

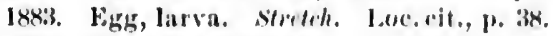

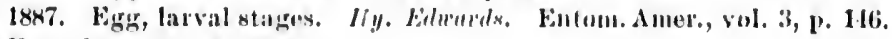

Foon l'laxts. Lionater', ath.

Orgyia vetusta. Boistl.

1881. Larva. Hy. Eithardy, Papilio, vol, 1, 1. 60.

Food Plicist. L."lpinur.

Orgyia gulosi. Hy, Eilwards.

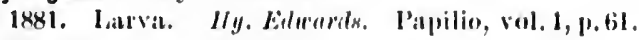

Foob l'isist. Quncele.

Orgyia Icucostigma. A h.,-siul.

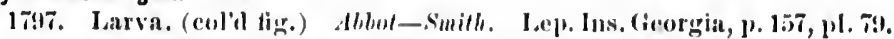

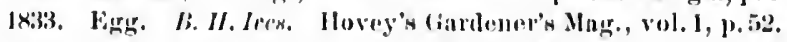

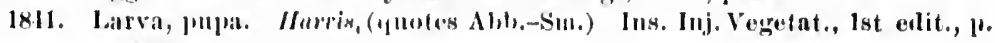
$21 \%$.

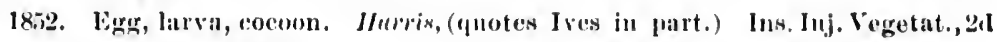
etlit., p. 281.

18it. Larva, (col'al tig.) Eumous, Nat. Hist. N. York, vol.5, 1. 230, pl.37.

1856. Larval, cocom, pmpa. Lïth. 2ll Rept. Lus. N. Yurk, p. 210.

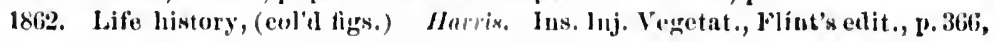
il. 7 .

1862. Larva. Morris, (ynotes IIarris.) Synop. Lep. N. Amer., p. 219.

1861. Larva, (tig.) Jaryer. Lif'o N. Amer. lns., p. 169.

18199. Life history, (tigs.) liley. 1st Nissomil hept., p. 114.

1871. Life history, (figs.) Le Rirrom, (after Riley.) 2ol Illinois Rept., p. 13.

1871. Forg, larva. Stunders. Canat. Butum, vol, 3, p. 15.

1871. Larva, cocoofi, pupa, (fign.) Brflume. Lepht. Entom. Soe. Ontario, p. 354.

1874. Egge, larva, pupa, (lign.) Sunuders. Rept. Burtom. Soc. Outario, p. 19.

1877. Larva, (tig.) Firnch. 'Trans. Dept. $\Lambda$ gr. Ill., vol. 15, p. 1xi.

1880. Larva. Fench, (as O. leneographa.) fith Rept. S. Ill. Norm. Vniv., P. H.

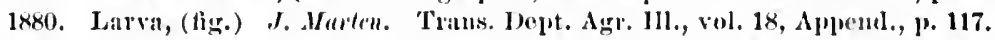

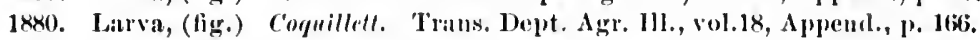

1881. Larva, cocoon, pupa, (figs.) Pucliurd, (after Riley.) Ins. Inj. Forest 'l'rees, j. 239.

1883. Lifo history, (ligs.) Sunuder.x. Ins. huj. Finits, P. 58.

1885. Life history, (tigs.) Lintucr. 2d Rept. State kintom. N. Yurk, p. 70.

18*7. Life history, (figs.) Riley. 13nll. 10, Div. Lintom. U.S. Dept. Agr., pp. 23-28.

1888. Larva, (fig.) Linluer. Ath liept. N. Y. State Entom., p. 48.

Foon Plants. Aeer, P'yrus, Querewn, cte.

Orgyia definita. Packard.

1888. Larva, notes on. Linfuer. Ath Rept. N. Y. State Entom., p. 50.

Parorgyia leucophæa. Abl.-Sm.

1797. Larva, (col'd fig.) Abbol-Swill. Lep. Ins, (ieorgin, p. 155, pl, 78.

1841. Larva. Harris. ('notes Abl,--Suı.) Ins. Inj. Vegretat., 1st edit., p. 264.

Food Plast. Querens.

Parorgyia clintonii. S. ant R.

1880. Larva. Coquillett. Canatl. Entom., vol. 12, p. 45.

1880. Larva. Coquillett. Trals. Dept. Agr. Ill., vol. 18, Append., p. 165.

food Plant. Quercus. 
Parorgyia achatina. Ablo,-Sin.

1797. Lalvil, (col'd tig.) Abbotl-simith. Lep. Ius. Georgia, 1. 153, pl. 77.

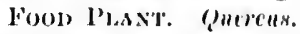

Parorgyia parellela. U. ant R.

1872. Latril. Linfuer. 26th Rept. N. York State Cab. N. Hist., p. 129.

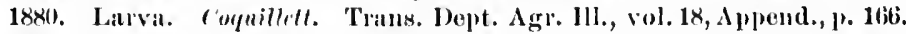

1ه81. Larva. I'uckarl, ('quotes Lintner.) lns. luj. Forest 'T'rees, p. 204.

18x:3. Lall'va. Samulers. Ins. Hoj. Fromits, p. 179.

1487. Life history. O. Seiffert. Eutom. Amer., vol.3, p.93.

Foon Plasts. I'yrux, Prumu, . Esculus, ete.

Lagoa crispata. l'ickitril.

1861. Laryi, cocom, pupa. Packard. P'roc. Eutom. Soc. I'hilat., vol.3, p. 333.

186ib. Lalra, (brief.) 13. I). Walsh. Practieal kutom., rol. 1, 1. 126.

18ti9. Larva, (fig.) Paclatrd. Guide to Studly of Ins., p. 288.

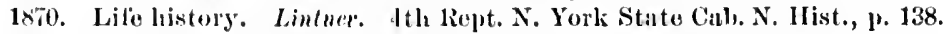

1881. Larva, cocoon, (hidef.) I'dekiarl. lns. Inj. Forest Trees, p. 176.

188.5. Larva, (tig.) I'ackard. Amer. Naturalist, vol. 19, p. 714.

Foon I'sasers. Ulmus, Myrica, Pyrm, te.

Lagoa opercularis. Albh,-Sm.

1797. Larva, (col'd fig.) Ablot-Swith. Lep. Ins. (icorgia, p. 105, pl. 53.

16.1. Larvil. Harris, (quotes Ahb.-Sm.) lis. Inj. Vegrctat., 1st odit., p. 261.

1856. Larva. Filele, (? crispata.) Bd Rept. Ins. N. York, p. 363.

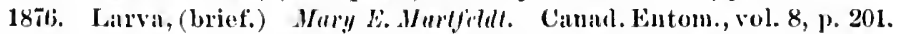

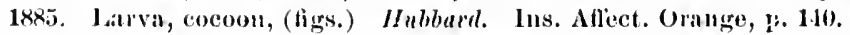

188x. Life history, (hrief,) (figs.) Lintuer, (jnotes Ilubbartl.) 4th Rept. N. Y. State Eutom., 1. 51.

Foon P'saxts. Cilmu, I'yrus, ite.

Lagoa pyxidifera. Abb.-sm.

1797. Larvi, (bold fig.) Abbot-Smith. Lep. Ins. Georgia, p. 107, pl. 5 l.

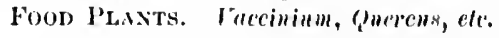

Sub-fun. Cochliopodæ.

Euclea cippus. Cram. (Qnerceti. H. Sel.)

1797. Larva, (col'd tig.) Ablot-Smith. Lep. Ins. Georgia, p. 145, pl. 73.

1811. Lanvis. Iltrrix, (quetes $\Lambda$ 131,-Sm.) lus. Inj. Vegetat., 1st ellit., p. 303.

18is. Larvat. Harrix. Ins. Inj. Vegetat., 2 ollit., p. 323.

18:8. Larva, (col'el tig.) Juncen. Natural. Libary, vol. 22, p. 178, pl. 21.

18t2. Larva. Haris. lns. Inj. Vegetat., Elint's odit., 1. 421.

18i2. Larvit. Momis, ('potes Dme:an.) Synop. Lep. N. Amer., p. 126.

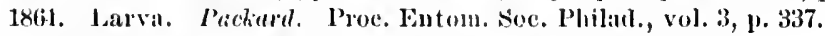

1869. Larva, (lig.) Harris. Entom, Corres., p. 17t, pl. 2.

1878. Larva. Ir. M. Amlen's. Psyche, vol. 2, p. 272.

18x1. Larva. French. l'ilpilio, vol. 1, p. 14n.

Foon P'laxts. Promus, P'yrus, Salix, etc.

Euclæa pænulata. Clem.

1881. Larva. French. Papilio, vol. 1, p. 14.

Foon Prant. Sulia.

Parasa chloris. H. Sch.

1864. Larva. T. Reakirt. Proe. Entom. Soc. Flhilad., vol. 3, p. 251.

1875. Lavia. A. G. Wetherby. Cincinnati Junr. Sc., vol. 2, 1.368.

1878. Larva. W. 1. Amlenes. Psyehe, vol. 2, p. 2iil.

1883. Larva. Silludeds-Elliwt. Papilio, vol, 3, p. 128.

1885. Larva. Fivencle. Canal. lintom., vol. 17, p. 160.

Forid Pidnts. Thmus, Myricu, ete.

Parasa fraterna. (ir.

1887. Larva. IIy. 'illurails. Eintom. Amer., vol, 3, p. 169,

Food l'lants. Cirishle, etc. 
Nochelia tardigrada. Clem.

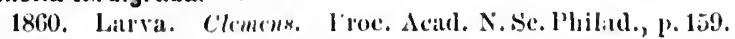

1862. Larva. Jorris, (^notes Clenens.) Synop. Lep. N. Amer., p. 131.

Foon Pi.axt. Ifwicot, (Clemens.)

Empretia stimulea. C'lesin.

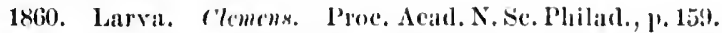

1862. Larva. Morrix, (fuotes Clomerss.) Syuop. Lep. N. Aner., p. 180.

1869. Lanva, (hig.) Horris, (as Lim. "phlipplatus.) Entom. Corres, p. 175, pl.2.

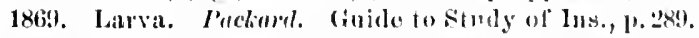

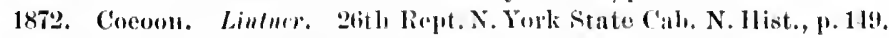

18iti. Larva. A. G. Wrtherby. Cincin.Jour. Sc., vol.2, p. 869 .

1877. Larvit. French. Trans. Dept. Agrr. Ill., vol. 1., p. 187.

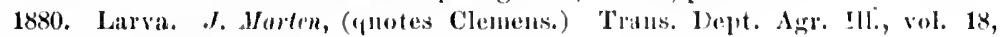
Appent., p. 117 .

18:3. Larva, cocoon, (figs) Sunders. Ins. Inj. Fonits, p. 113.

1885. Larva, cocoun, (cold figs.) H. G. Hoblow. Ins. Afloct. Olange, p. H1..

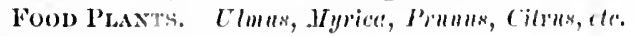

Phobetron pithecium. Alh.-Sin.

1797. Larva, (col'd figr.) Ablot-Smith. Lep. Ins. (ieorgia, p. 117, p].74.

1811. Larva. Marrix. Ins. Inj. Vegetat., lst edit., p.sol.

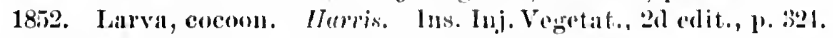

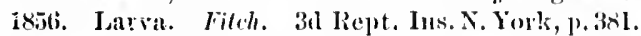

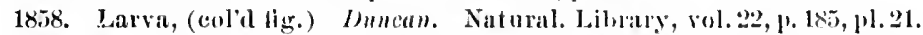

1862. Larva, cocoon, (lig.) IIarris, Ins. Inj. Yegelat., Fliut's edit., 1. 12I.

1862. Larva. Morvis. Svmop. Lej. N. Amer., p. 127.

1872. Cocoon. Lintuer. 2bth Rept. N. York State Cah. X. Ilist., p. 149.

1881. Larva. P'tcliturl. Ins. In,j. Forest Trees, p. 47.

1883. Latva, eoeoon, (tig.) Sentulers. Lns. Lnj. Fruits, p. 112.

1885. Larva, eocoou, (col'd figs.) IT. G. Hubhert. Ins. Atheet. Orango p. 113

Foob Plants. Prumis, Concrens, ate.

Phobetron hyalinum. Wilsh.

1863. Larva. I, I). I'alsh. Proe lioston soc. N. Ilist., vo1. 9, 13. 297.

Foon Plast. I'rumix.

Limacodes scapha. Harris.

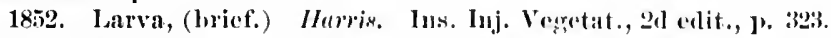

1862. Larva. Harris, Ins. Inj. Vegetat, Flint's mit., 1. 420.

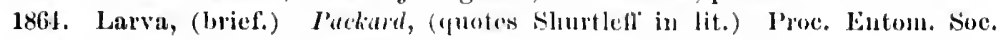
Plitiml., vol. $3,1.311$.

1869. Larva, (fig.) Harris. Fifom. Corres., ple. 176, 300, pl. 3 ,

1873. Larva. Stretch, (quotes Ilarris.) Kygann. linmbye. N. Amer., p. 2017.

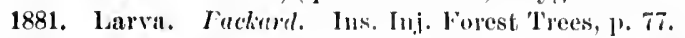

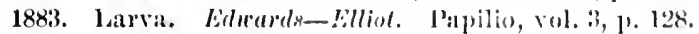

1885. Laurva, (col'd fig.) H. G. Hnbbarl. Ins. Alleet. Orange, p. 14.

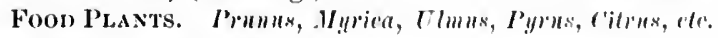

Limacodes biguttata. l'ickard.

1873. Cocoon. Stretch. Zyigru. Bombye. N. Amer., p. 202.

Limacodes fasciola. 1I. Selı.

1860. Larva. Clemens. I'roc. Acat. N. Sc. Plsilatl, 1. 157.

1862. Larva. Morrix, (quotes Clemens.) Synop. Lep. N. Amer., p. 128.

1872. Larva, (brief.) Lintuer. 26th liept. N. York State Cal. N. Ilist., p. 199.

1881. Lierva. Puckurd, (quotes Clemens.) Ins. luj. Forest Trers, l1. 112.

1883. Larva, cocoon, (Inrief.) Saumder. Ins. Inj. Frnits, p. 179.

Fom Plaxt. Aecr.

Nork.-These deseriptions include L. laticlavia, Clem, which I heliove to be 2 listinct npecies.-II. E.

Bur.L. 35-5 
Sisyrosea inornata. (4. and R.

1887. Larvi. (i. D. Hulst. Entom, Amer., vol, 3, 1. 66.

1889. Latra. II. (i. byut. Canal. Entom., vol. 21, p. 77.

Foon I'last. Myrica cerifera.

Adoneta spinuloides. 11. Sclı.

1860. Latrva. Clemens. Proe. Aeal. Sc. Plilaul., p. 168.

1862. Larra. Morris, (quotes Clemens.) Synop. Lep. N. Amer., 1. 129.

1882. Larva, Mis. Ballact. Papilio, vol. 2, 1. 83.

1883. Lal'va. Edtermils-Elliot. Papilio, vol. 3, p. 129.

Foon Plast. Pruulus, ete.

Packardia fusca. I'ackard.

1861. Cocoon, pupa. I'ackard. P'roc. Entom. Soc. Philad., vol. 3, p. 313.

Kronæa minuta. Reakirt.

186t. Larva, pupa. T. Reukirt. Proe. Entom. Soc. Philat., vol. 3, p. 251.

Sul-fanl. Psychidæ.

Psyche confederata. Grote aud Rol.

1868. Lanval caso, (fig.) Grote-Rohinson. Trans. Amer. Entom. Soc., vol. 2, 1. 191, pl. 3.

1885. Larval ease, (fig.) H. G. Irubburt. Ins. Affect. Orange, p. 118.

1887. Larva, pupa. Iry. Erlecarels. Entom. Amer., vol. 3, p. 168.

Psyche fragmentella. IIy. Edwards.

187ti. Larval case. Hy. Edurarls. Proc. Cal. Acat. So., Novemlser.

Psyche coniferella. Hy. Elwarls.

1876. Larval case. My. EAturivels. Proe. Cal. Acari. Sc., Novemlier.

Platceticus gloveri. Packard.

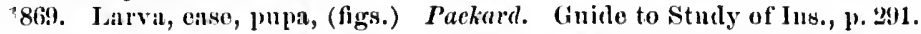

1883. Larva, case, pupa, (figs.) Suunders, (after Packard.) Ins. Inj. Fruits, p. 380.

1885. Case, pnipa, (figs.) IT. G. Hubbard. Ins. Aftect. Orango, p. 149.

Food Pi.ant. Citrus.

Thyridopteryx ephemeræformis. Haworth.

1853. Larva, case. Harris. Downing's IIorticnlt., vol. 8, p. 461, (as Oik. coniferarmin.)

1855. Larva, ease, pupa. T. Glocer. Trans. N. York State Agrr. Soc., p. 79.

1859. Larva, case. IJ. $I$, Gosse. Letters from Alabamia, 1.283.

1861. Case. l'uckurel. Proc. Entom. Soe. Philad., vol. 3, p. 351.

1866. Larva, case. B. D. Walsh. Pratetical Entom., vol. 2, pp. 22, 8.1.

1866. Larva, caso. S. S. liathron. Loc. eit., p. 53.

186\%. Life history, (figs.) liil'y. 1st Missonri Rept., p. 147.

1869. Larra. Havis, (as Oik. coniferarmu.) Lintom. Corres., lp. 176, 300.

1869. Larva, case, (fig.) Packird. Guide to Study of Ins., p. 291.

1877. Larva, case. French. 'Trans. Dept. Agr. Ill., vol. 15, p. 187.

1880. Egg, larva, case, pupa, (figs.) J. Marten. Trans. Dept. Agr. Ill., vol. 18, Append., p. 118.

1881. Larva, case. Packart. Ins. Inj. Forest Trees, 1 . 248.

1881. Life history. Helen S. King. Payche, vol. 3, p. 241.

1883. Young larva. Hy. Efluards. Pipilio, vol. 3, p. 2.1.

1888. Lifo history, (figs.) Saumers. Lus. Inj. Frnits, p. 222.

188.). Larval case, pupa. H. G. Hublard. Ins. Affect. Orange, p. 147.

1886. Life history. W. MreFulenel. Jonr. Trenton N. II. Soc., vol. 1, p. 1.

1887. Lifo history, (figs.) Riley. Bull. 10, Div. Entom. U. s. Dept. Agrr., pl. $23-28$.

1888. Fggr, larval case, Iarra, pupa, (figs.) Grote. 18th Rept. Futom. Soc. On. tario, p. 74.

Foop P'tavts. Pinus, Cuprersus, Salir, (Muevens, rte. 
Thyridoptery meadii. IIy. Fdwards

1881. Larval ease. Hy. Elluar

upilio, vol 1, 1. 116.

Oiketicus abbotii. Grote.

1880. Larva, ease. Grote. N. Anter. Entom., p. 52.

1885. Life history, (figs.) H. G. IInbhard. Ins. Atlect. Orange, p. 14, pl. 12.

Food Prants. Citrus, etc.

Oiketicus davidsonii. IIy, Edwards.

1876. Larval ease, pupis, (figs.) Hy. Eidwarls. Proc. Cal. Aead. Sc., Noventber.

Perophora melsheimeri. Harris.

1841. Larva, ease, pupa. Ifurris. Ins. Inj. Vegetat., 1st edit., p. 301.

1852. Larva, ease, pupa. Iluris. Ins. Inj. Vegetat., 2ll edit., p. 319.

1862. Larva, case, p"1 pa, (figs.) Harris. Ins. Inj. Vegetat., Flint's edit., p. 415, pl. 6.

1869. Larva, case, pupa. Harris. Entom. Corres., p. 151.

1869. Iarva, easo. Puckard. Guicle to Study of Ins., p. 292.

1880. Larva, ease. Coqmillett. 'Trans. Dept. Agr. III. ol. 18, Append., p. 156. Foon Plant. Quereles.

Ichthyura inclusa. IIülun.

Sub-fam. Notodontidæ.

1797. Larva, (eol'd fig.) Abbot-Smith, (as Clos. anastomosis.) Lep. Ins, Georgia, p. $143, \mathrm{pl} .72$.

1841. Larva. Hurris. Ins. Inj. Vegretat., 1st edit., p. 314.

1852. I.arva. IIarris. Ins. Inj. Vegetat., 2d edit., !. 333.

1858. Larva. Fitch. 5th Rept. Nox. Ins. N. York, p. 845.

1862. Life history, (col'd figs.) IIarris. Ins. Inj. Vegetat., Flint's edit., p. 431.

1869. Larva, (fig.) Harris. Entom. Corres., p. 3i0, pl. 3.

1881. Larva, pupa. Packud, ('juotes Harris.) Ins. Inj. Forest Trees, p. 122.

1883. Yonnig lar'a. Hy. Edwarts. Papilio, vol. 3, p. 24.

Food Plant. Topulus.

Ichthyura ornata. S. and $\mathrm{R}$.

1885. Lifo history. Freuch. Canad. Entom., vol. 17, p. 248.

Food Prant. Salix.

Ichthyura palla. Frencli.

1882. Larva. French. Canad. Entom., vol, 14, p. 3.1.

1885. Life history. French. Canad. Eutom., vol. 17, p. 41.

Food Plant. Salix.

Ichthyura albosigma. Fiteh.

1855. Egg, larva, cococn. Fitch. 2ll Rept. Ins. N. York, p. 274.

1858. Larva. Fiteh. 5th Rept. Ius. N. York, p. 8/4.

1881. Larva. Packard, Ins. Inj. Forest Trees, p. 122.

Foon Plant. Populus.

Apatelodes torrefacta. Albb,-Sm.

1797. Larva, (eol'd fig.) Abbot-Smith. Lep. Ins. (Georgia, p. 151, pl. 76.

1864. Larva, (brief.) Packard. Proc. Entom. Soc. Pisilad., vol. 3, p. 353.

1869. Larva. IIarris. Eintom. Corres, p. 307.

Food Plants. Rubur, etc.

Apatelodes angelica. Grote.

1872. Larva. Lintner. 26th Rept. N. York State Cali. N. Hist., p. 130.

Foon Prants. Fraxinns, Syringa.

Datana ministra. Drury.

1797. Larva, (col'l fig.) Abbot-Smith. Lep. Ins. Goorgia, p. 161, pl. 81.

1841. Larva. Harris. Ins. Inj. Vegetat., 1nt ellit., p. 312.

1852. Larva. Harris. Ins. Inj. Vegetut., 21 elit., p. 332.

1855. Larva, pupa, (figs.) Fiteh. 2il liept. Ins. N. York, p. 237.

1856. Larva. Fiteh. 3d Rept. Ins. N. York, p. 337. 
186i2. Larvi, (tig.) Harris. Ins. Inj. Vegretat, Flint's edit., pr. 129.

1866. Lalrva. (irote-Robinson, (quote Angus in lit.) Proc. Lintom. Soc. l'hilad., vol. 6,1 . 11.

186il. Larva. B. D. Walsh. Practical Entom., vol. 2, p. 7.

18i99. Larva, (lig.) IJarris. Butun. Corres., p. 308, pl. 2.

1873. Lifo history, (tigs.) Le Barou. 4th lllinois Rept., pr. 186.

1877. Latrva, (tig.) French. 'Tlaus. Dept. Agr. Ill., vol. 15, p. 189.

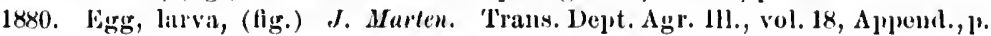
119.

1880. Lygg, larva, (tig.) Coquillett. Tranı. Dept. A gr. Ill, vol. 18, Append., p. 167.

18*3. Lall'va, (fig.) Sunnde's Ins. Inj. Fruits, p. 61.

1888. Life history. W. Beulenmiiller. Canad. Entom., vol. 20, p. 16.

Foon Plaxts. C'urya, Juglans.

Datana angusii. G. and R.

1866. Larva. Grotc-Robinson, ('note Angus in lit.) P'roc. Entom. Soc. I'hilad., vol. 6, p. 10.

1888. Larva. II. Bentenmiiller. Canad. Entom., vol. 20, p. 135.

Foon l'rasts. Juglans, C'arya.

Datana major. (i. and $\mathrm{k}$.

1878. Larvis. IT. V. Audrew's. Psyche, vol. 2, p. 272.

1889. Latva. II. G. Dy.u. Canaul, Entom., vol, 21, p. 31.

Food Plast. Andromeda ligustrina.

Datana integerrima. $G$, and $\mathrm{k}$.

1866. Larva. Grotc-Robinson, ('1noto Angus in lit.) Proc. Entom. Soc. Philad., vol. 6, p. 13.

1888. Larval stages. IT. Beutermiiller. Canal. Entom., vol. 20, p. 134.

Food Plants. Jutjlans, C'urya, (bucrecus, ctc.

Datana floridana. Graef.

1881. Larva. A. Koebcle. Bull. Brooklyn Entom. Soc., vol. 4, p. 21.

Datana drexelii. Hy. Edwarls.

1881. Larva. Hy. Edwath. Papilio, rol. 4, p. 25.

1886. Life history. W. Bentrumïller. Cantud. Lintom, vol. 20, p. 57.

Food Plasts. Iaccinium corymbosum, Hamamelis.

Datana contracta. Walker.

1866. Larva. Grot-liobinson, (đuote Angus in lit.) Proc. Entom. Soc. Philad., vol. 6, p. 14.

1888. Larval stages. H. Benlenmiiller. Cauad. Entom., vol. 20, p. 13.

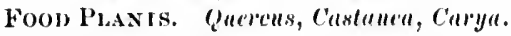

Datana perspicua. G. iod R.

1887. Eggr, larval atages. Hy. Riludeds. Fintom. Amer., vol, 3, p. 170.

Foon Plast. An typhina.

Nadata gibbosa. Abb,-Sm.

1797. Larva, (col'll tig.) Abbot-Suith. Lep. Lus. Georgial, p. 163, pl. 82.

1869. Larva. Harris. Eintoni. Corres., p. 308.

1872. Larva, (fig.) Lintuer. 26th liept. N. York Stato Cab. N. IIist., p. 150.

187.1. Larva, (fig.) l'uckarl, (quotes Lintner.) Aner. Naturalist, vol. 8, p. 691. Foon Plante. Queveres.

Hyparpax aurora. Alob.-Sm.

1797. Larva, (col'd fig.) Abhot-Smilh. lep. Ins. Georgin, p. 173, pl. 87.

1888. Larval stages. Hy. Einurils. Entom. Amer., vol. 3, p. 169.

Foon Plant. Queveus.

Gluphisia trilineata. l'ackard.

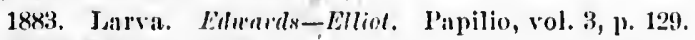

Foon Plant. Sulix. 
Notodonta stragula. (irotre.

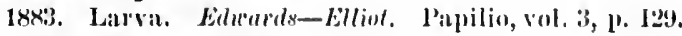

Food l'LaNT. Sulix.

Lophodonta angulosa. $\Lambda$ bl, - Sm.

1797. Larva, (col'd tig.) Abbot-Smith. Lep. Ins. Georgia, p. 1fí, pl. 83.

Food Plast. Qurrens.

Pheosia rimosa. Park.

1882. Larva, pupa. C. F. Goodhue. Canad. Entom., vol. 14, p. 73.

Foon Puants. Sulix, Popmlus.

Pheosia californica. Stretch.

1873. Larva, (col'd fig.) Strech. \%yginn. Bombyc. N. Amer., p. 117, pl. 10.

Edema albifrons. Alb.-Sul.

1797. Larva, (col'd fig.) Alhot-Smilh. Lep. Ins. Georgiti, p. 159, pl. 80.

1854. Larva, pupa, (tigs.) Émmons. Nat. Ilist. N. York, vol. 5, p. 212, pl. 37.

1869. Larva. Harris. Eutont. Corres., p. 304.

1877. Larva. Freuch. Trans. 1)ept. Agr. 1ll., vol. 15, p. 1!1.

1880. Larva. J. Murten. Trans. Dept. Agrr. Ill., vol. 18, Append., p. 120.

Foon Plant. (') 'urecte.

Seirodonta bilineata. Packard.

1869. Larra, (tig.) Harris. Entom. Corres., p. 201 , pl. 1.

1869. Larva, (fig.) Harris, (as (ihuph, uhui.) Lase cit., p.302, pl. 11.

1886. Larva. Freuch. Canad. Entom., vol. 18, 1. 49.

Food Plant. Ulmus.

Oedemasia concinna. Ah, -Sm.

1797. Larva, (eol'il tig.) Albiot-Smith. Lep. Ins, Georgia, p. 169, pl. 85.

1811. Larva. Harris. Ho. luj. Vegretat, 1st edit., p. 309.

1852. Egg, larva. IIteris. Ins. Inj. Vegetat., 2 I edit., p. 328.

1856. Larva. Fitrle. 3d Rept. Ins. N. York, p. 3 le.

1862. Egg, larva, (tigs.) Iluris. Ins. Lnj. Vugetat., Flint's edit., p. 426.

1869. Larva, (tigr.) Honris. Entom. Corres., p. 303, pl. 1.

1877. Larva. Fruch, (quotes Itarris.) 'Trans. Jept. Agr. Ill., vol. 15, p. 190.

1880. Larva. J. Marten. Thans. Dept. Agr. Ill., vol. 18, Appenti, p. 12.9.

1881. Lifo history. Stunuldr.x. Canad. Entom., vol. 13, 1. 138.

1882. Larra, pupa, (tigss.) Sanuders. 12th Rept. Entom. Soe. Ontario, p. 21.

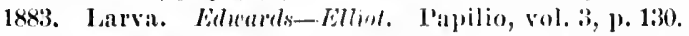

1883. Larva, pupa, (figs.) T. .J. Eitgr. Rept. Agr. Pennsyly., p. 70.

1883. Larva, pupa, (figs.) Sormilers. Lns. Inj. Fruits, p. li3.

1888. Larva, (tir.) J. Flithery. Rept. Entom. Dept. Agr'. Canad., p. $2 x$.

Foon P'lant. Salir, I'rmuns, the.

Oedemasai salicis. Hy. Edwards.

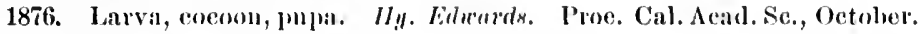

Food Plant. Solir.

Dasylophia anguina. Allb.....m.

1797. Larva, (eol'd fig.) Ablit-smith. Lep. Ins. Georgia, p. 167, pl. 81.

1869. Larra, (fig.) IIarris. Eutom. Corres, p. 301, pl. 1.

1889. Lifo history. H. (i. Dyar. Lintom. Amer., vol, 5, 1. 5..

Foon Plant. Roliniat.

Coelodasys unicornis. Alim.-Sm.

1797. Larva, (eol't tigr.) dibot-Smilh. Lep. Ins. Georgin., P. 17f, pl. 83.

1841. Larva. Herris. Ins. Inj.j. Vegetat., 1st edit., p. 307.

1852. I orva. Harris. Ins. Inj. Vegetat., 2 d edit., p. 326.

1856. Larva. Fildh. 3il Rept. Ins. N. York, p. 363.

1862. Larva. Harris. Ins. lnj. Vegetit., Flint's eelit., l. 42.1.

1869. Larva, (fig.) Horris. Entom. Corres., p. 302, pl. 11.

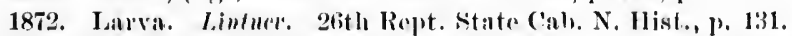

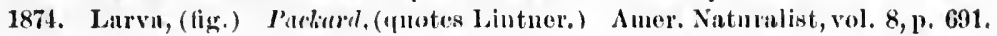


1877. Latra. Iirurle. 'Trans. Dept. Agr. Ill., vol. 15, p. 191.

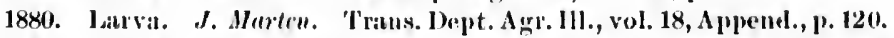

1880. Larva. Compillett, (quotes Lintner.) 'Irans. Lept. Agr. III., vol. 18, Append., p. 147.

1880. Larva. Coquillett. Trans. Dept. $\Lambda$ gr. Ill., vol. 18, Append., p. 181.

1883. Larva, cocom, (figs.) Samulers. Ins. Inj. Fruits, p. 80.

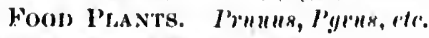

Coelodasys mustelina. Parek.

1886. Larva. Freurl. Canad. Entom., vol. 18, p. 92.

Foon) Plant. Liogr.

Coelodasys biguttata. Pack.

1870. Larva, pupa, (figs.) I'urliard. Amer. Naturalist, vol. 4, p. 229.

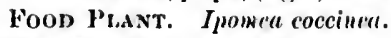

Janassa lignicolor. Walker.

1889. Lifo history. H. G. Myar. Entom. Amer., vol. 5, p. 91.

Heterocampa astarte. Domllil.

1869. Larva. Hurvis, (ґnotes Donlleday in lit., 1839.) Lintom. Corres., p. 132.

Heterocampa unicolor. Iack.

1887. Larva. Hy. Lidurterlw. Entom. Amer, vol, 3, p. 108.

Food Plant. Acrer prento-pllantenus.

Heterocampa pulverea. (1. and $\mathrm{R}$.

1880. Larva. Frruch. Canal. Entom, vol. 12, p. 83.

1880. Larva. Firuch. Gth $\Lambda$ m. Rept. S. Ill. Normal Univ., p. 44.

1881. Larva. I'delidird, (đnotes l'rench.) Ins. Inj. Forest 'Trees, p. 46.

Foon Plant, Quercus.

Heterocampa marthesia. Cran.

1884. Iarva. P'reliarl, (as II. tessela.) Amer. Natnralist, vol. 18, p. 1045.

Foon Plant. Querens.

Cerura occidentalis. Lintn.

1881. Larva. French. Canad. Entom., vol. 13, p. 144.

Foon Plant. Cerasus.

Cerura cinerea. Walk.

1883. Larva. Elwarls-Elliot. Papilio, vol. 3, p. 130.

Food Prant. Sulix.

Cerura borealis. Boisd.

1797. Larra, (col't figs.) Abbot-Smith, (as C. furenla.) Lep. Ins. Georgia, p. 141, pl. 71 .

1811. Larva. IIarris. Ins. Inj. Vegetat., 1st edit., p. 305.

1872. Larva, (fig.) Liutuer. 26th Rept. N. York Stato Cab. N. Hist., p. 151.

1874. Larva, (fig.) I'uckiarl, (quotes Lintner.) Amer. Naturalist, vol. 8, p. 692.

1881. Larva. French. Canal. Entom., vol, 13, p.115.

Foon I'lavt. Sulix.

Dryopteris rosea. Walk.

\section{Sul-fam. Saturnidæ.}

1887. Larva. Grote. Canad. Entom., vol. 19, p.50.

Food Plant. Viburuum acrifolium.

\section{Drepanodes arcuata.}

1889. Larva. W. Beuteumü̈ller. Entom. Amer., vol, 5, p. 38.

Sericaria mori. L. (Silk-worm, domesticaterl.)

1836. Life history, (figs.) Treatise Silk Manufuct., (Larduer's Cyclop.)

1840. Larva, pupa, (lig.) Wertweod. Intro. Modl. Class. In., vol. 2, p. 378, fig. 105.

1857. Lifo history, (figs.) Chenn-Demarts. Encye. Hist. Nat. Papillons, vol.2, pp. 7-16.

1863. Larva, cocoon. W. F. Kivlly. Furop. Butt. and Moths, p. 133.

1864. Lifo history. Jaeger. Lifo N. Amer. Ins., p. 145. 


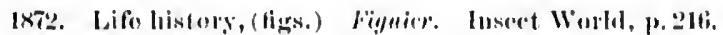

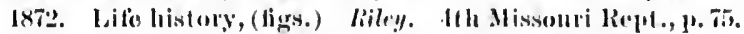

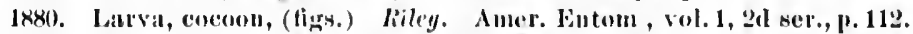

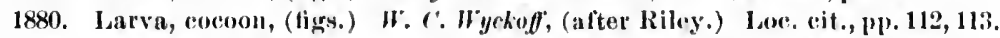

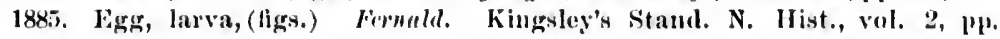
$43.4,460$.

1880. Life history, (figs.) Riley. Dept. Agr. Bull. No.9.

Foon Plasiss. (ogage Orange, Rubers, ete., (Morris.)

Actias luna. 1.

1797. Iarva, (col'd tig.) Albot-smith. Lep. Ins. (ycorgia, p. 95, pl.48.

1837. Larva. Wexteood, (, (uotes Ahl.-Sm.) iu Drury 1ll. Exot. Entom., vol.1, ए. 15.

1841. Iarva, pupa. Marris. Ins. Inj. Vegetat., 1st edit., p.277.

18i2. Larva. IIarix. Ins. Inj. Vegetat., 2 l edit., p. 297.

1851. Larva, pupa, (col'u lign.) Emmows. Nat. llist. N. York, vol. 5, p. 233, pl. 39.

18ij6. Inava. Fitch. 3i Rept. Ins. N. York, p. tis.

18.9. Larva, cocoon. ('lemens. Jour. Acall. Se. P'hilad., July.

186:. Lalva. Morris. Synop. N. Amer. Lep., p. 2.5.

181;2. Larva, cocoon. IIamix. Ins. Inj. Vegetat., Flint's edit., p. 382.

1865. Cocoon, (brief.) Grote. Amer. Entom., p. 13.

1867. Isarva. Tenney. Nat. Ilist., p. 401.

1869. Eug. Minot. Canar. Eutom., vol. 2, p. 27.

1869. Larva, (fig.) Harris. Entom. Corres., p. 293, pl. 4.

1872. Lifo history, (figs.) Riley. Itlı Missonri Rept., p. 123.

1872. Egg, larval stages. Linluer. 26th Rept. N. York Stato Cab. N. Hist.p. 126.

1874. Larva, (fig.) J. G. Wood. Insects Albroad, p. 674.

1874. Larva. Gentry. Canal. Entom., vol, 6, p. 86.

1875. Lifo history. L. r. liogers. Canad. Entom., pp. 141-199.

1875. Egg, larva, cocoon. R. V. liogens. Rept. Entom. Soc. Ontario, p. 43.

1877. Larva. Freneh. Truts. Dept. Agr. Ill., vol. 15, p. 192.

1880. Larva. "J. Morlen. T'rans. Dept. Agr. Ill., vol. 18, Append., p. 124.

1880. Larva, (ig.) Coquillett, (ynotes Lintner.) 'Trans. Dept. Agr. Ill., vol. 18, Append., 1. 178.

1881. Larva, (brief.) Paclard. Ins. Inj. Forest Trees, p. iti.

1882. Larva, brief, (tig.) J. A. Moffat. Ropt. Eutom. Soc. Ontario, p. 29.

1885. Eggr, larva, cocoon. Frnald. Kingsloy's Stand. N. Ilist., vol. 2, p. 458.

Food Piants. Jigluns, Liquidambar, Betult, F'agus, te.

Telea polyphemus. Cramer.

1797. Larva, (col'd fig.) Abbot-Smith. Lep. Ins. Georgia, p. 93, pl. 47.

1810. Larva, (fig.) I'. I. Cosse. Canad. Naturalist, p. 309.

1811. Iarva, pupa. IIaris. Ins. Inj. Vegetat., 1st edit., p. 278.

1852. Larva. IJurvis. Ins. Inj. Vegetat., 2 d edit., p. 298.

18isl. Larva. Emmons. Nat. Hist. N. York, vol. 5, 1. 236.

18:\%. Larva. Fiteh. 3a Rept. Ins. N. York, 1. 45\%.

1859. Lawa, cocoon. Clemens. Jour. Acad. N. Sc. Philad., July.

1862. Larva. Morris. Synop. Lep. N. Amer., p. 2296.

186. Larva, cocoon. IIurris. Ins. Inj. Vegetat., Flint's edit., p. 381.

186 Tarva. Jaeger. Lifo N. Amer. Ins., p. 159.

187.- Lifo history, (figs.) Riley. 4th Missouri Rept., p. 125.

1872. Egg. Lintuer. 26tlı Rept. N. York State Cal). N. Ilist.,p. 152.

1874. Larva. Gentry. Canad. Entom., vol. 6, p. 86.

1875. Life history, (figs.) Saunders. Rept. Fintom. Soc. Ontario, p. 41.

1879. Larva, cocoon, pupa, (figs.) Mrs. Ballard. Insect Lives, p. 45.

1880. Larva. J. Murle $u$. Trans. Dept. Agr. Ill., vol. 18., Append., p. 124. 


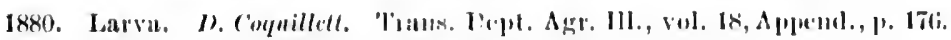

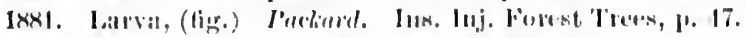

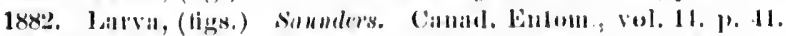

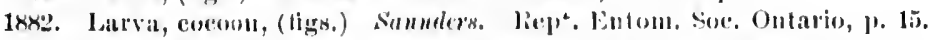

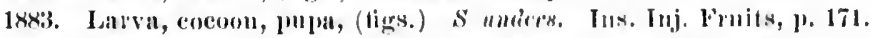

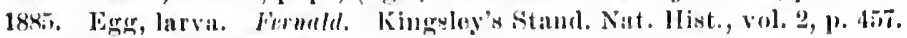

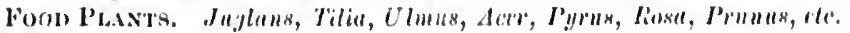

Attacus cinctus. 'Trpler.

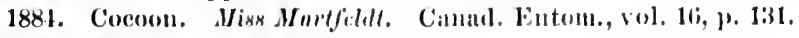

Attacus yama-mai (domesticated).

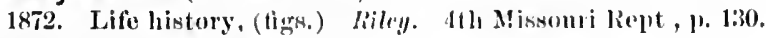

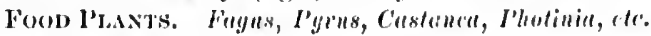

Atiacus cynthia. Drury.

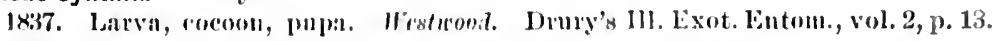

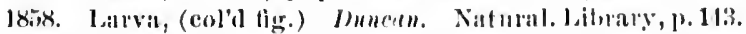

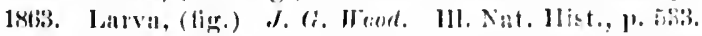

1872. Lege, larvia, coeoon. Hignior. Insect World, p. 21\%.

1872. Life history, (figs.) Liley. Ith Missonti liept., p. 112.

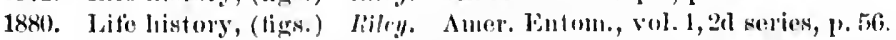

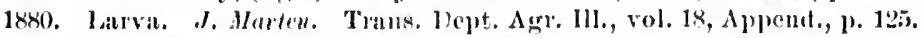

1885. Eggr, larva, (figs.) remeld. Kingsly's Stand. Nat. Il ist., vol. 2, p. 457.

Foon Piants. Ailunthus, Custor-beren.

Callosamia promethea. Drury.

17!). Latra, (col'd figs.) Allot-Swith. Lep. Ins. Georgia, p. 91, pl. 44.

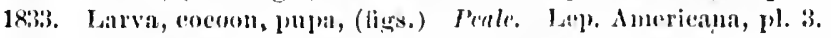

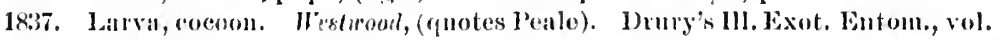
$2,1.21$.

1838. Larva, cocoou, pupa, (figs.) Westrood, (unotes Peile.) Introd. Eutom., 1. 102.

1811. Lava, pupa. IInris. Ins. Ini. Vegretat., 1st edit., p. 281.

1852. Larva, cocoon. Harris. Ins. Inj. Vegetat., 2d edit., p. 300.

18is. Egg, larva. L'mmons. Nat. IJist. N. York, vol. 5, 1. 288.

18is. Larva. Fitch. 3il Rept. Jus. N. York, p. 37it.

18:8. Larva, cocoon, pupa, (ligs.) Juncan. Nitural. Libnary, vol. 22, p. 136.

1859. Lavai, coeoon. C'temens. Jour. Acatl. N. Se. Thilatl, July.

1862. Ligg, larva. IIarris. Ins. Inj. Vegetst., Flint's edit., p. 391.

1862. Latva. Marvis. Synop. N. Amer. La']., 11. :21.

1861. Egg, larva, cocoon. ,aegre. life N. Mmer. Jus., p. 162.

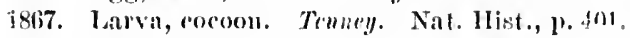

1872. Ligre, young larra. Lintner. 26th Rept. N. York State Calı. N. IFist, p. 126.

1872. lifo history, (figs.) Ril'y. th Missouri Rept., p. 12 .

1877. Larva. Fromeh, (quotes IItris.) Trans. Dept. Agr. Inl., vol. 15, p. 193.

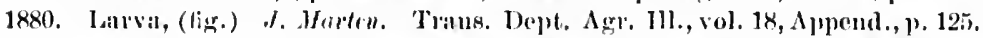

1880. Larva, (fig.) I). Coqnillett, (fuotes Ilarris.) Trans. Dept. $A$ grr. Ill., vol. 18, Appent., p. $17 \%$.

1883. Egg, lan'val stages, cocoon, (figs.) Saunders. Canal. Eutom., vol. 15, pl. 32, p. 231.

1883. Egrg, larval stages, cocoon, (figs.) Stumtr's. Ins. Inj. Fruits, p. 205.

Food Prants. Froxiuns, Sassafras, Liviotemtron, Liquidambar, deer, Prunus, P'yrus, ste.

Platysamia cecropia. I.

1797. Larva, (col'l fig.) Ahhot-Smith. Lep. Ins. Georgia, p. 89, pl.45.

1837. Lava, cocom. "Fistwool, (quotes Al, -Smith). Drury's Ill, Exot, Eintom., vol:1, 1.33.

18.1. Larva, pupil. Harris. Ins. Inj. Vegetat., 1st edit., p. 279. 


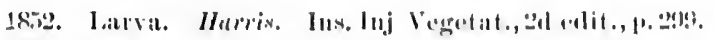

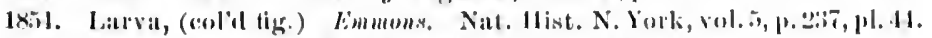

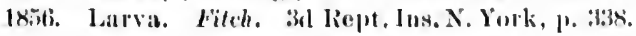

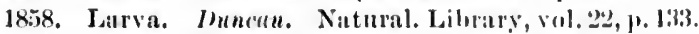

18:9. Iarva, coemm. Clemens. Jour. Arabl. N. Sr. l'hilanl., Muly.

186e. Iarva. Horris. Synop. N. Amer. I,ep.,p. 2ay.

1862. Larva, cocoon, pmpa, (ligs.) Ilevis. Ins. Inj. Verotat., l'lint's colit., p. $3 \times \div$.

1861. Iarva, cocont, (figs.) Jacyer. Jifo N. Amer. Ins., J. 1is.

1867. Iarva, jupa, (tigns.) Trmmey. Nat. Hist., l. Itot.

18til. Larva. Harris. lintom. Corres, p.29!.

1870. Vigg. I. T. Nimagne. Camal. Entum., vol.2, p.82.

1871. Larva, coconon. Suumelers. Camad. Butom., vol. 3, p. 119.

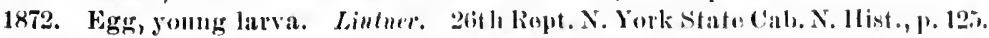

1S72. Lifo history, (ligs.) likly. Ith Missomi Rept, p. 10\%.

1874. Lifo history. (tigs.) Sammler. Rept. Linlom. Soc. Onlatio, 1.21.

1877. Lifo history. Gentry. Canarl. Eutum., vol. @, l. 11.

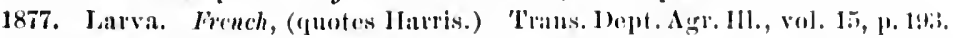

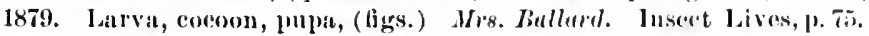

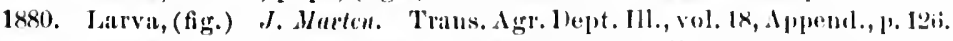

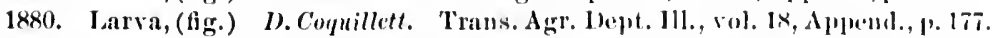

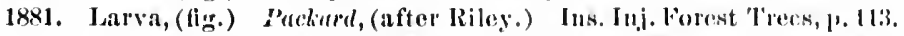

1881. Iatra, cocoon. (‥ D. IIulst. Bull. Rrooklyn Butom. Soc., vol. 1. J. 57, hybrid lotween 1'. cocopopia aml l'. cenuothi.

1883. Egg, larva, cocoon, (figs.) Sunnders. Jus. Inj. Fruits, 1. Ti.

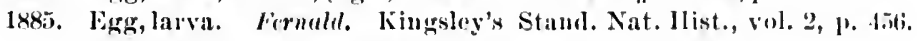

Foow I'i.Axts. Pyrus, l'runus, te.

Platysamia columbia. Smith.

1871. Cocoon. G.J. Boules. Canal. Eutomm, vol, 3, p. 2 . 3.

1878. Latrva, (col'd fig.) (i..J. Bonles. Canal. Entom., vol. 10, frontispiece.

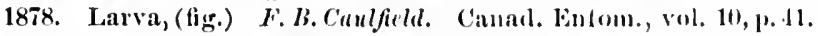

1878. Life history. Itrmald. Canal. Entom., vol. 10, 13.13.

187!). Larva, cocoon. IItge. Bull. Buffilo Soe. Nat. Itist., p. 201.

1880. Larva. D. Coquiletl, (quotes Caulfielel.) 'Traus. Dept. Agr. Ill., vol. I-, A ppend., p. 177.

Foon Plaxt. Latix americana.

Platysamia gloveri. Strecker.

1878. Cocoon, pupa. K. L. Graef. Bull. lirooklyu Eutum. Soc., val. 1, p. Ti.

Platysamia ceanothi. Belır.

1869. Larva, cocoon, pupa. Moisturul. Lep. Califur., 1.81.

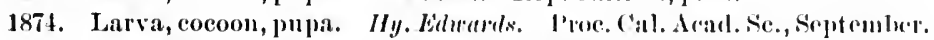

1876. Larva, cocoon, pupat. Strecker, (quotes lyy. lidwarls.) Jap. Rhopal. et IIeteroc., p. 103.

1880. Larva. A. Wuilly. Canall Butom, vol. 1:, 1.:207.

Foon Plaxt. Ceanothus thyrxiflorus.

Saturnia mendocino. Behrens.

1878. Egg, larval stages. IIy. Edwards. P'oc. Cal. Acarl. Sc., Jannary.

Foon Pr.sxt. Aretostaphylos tomentost.

Saturnia galbina. Clemens.

1888. Egg, young larva; cocom, pupa. IIy. Eibuerts. Eutom. Amer., vol. I, p. 61 .

Psuedohazis eglanterina. 13ois.

1833. Larva, (col'd figr.) Titian I'etle. Lrp. Amerieana, pl.2.

1875. Egg, larva. Hy. Eilutrils. Proc. Cal. Acarl. Se., $\Lambda$ pril.

Food Plants. lihummus, hosa. 
Hemileuca maia. Drury.

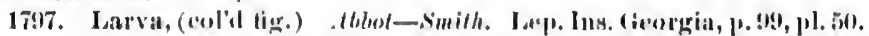

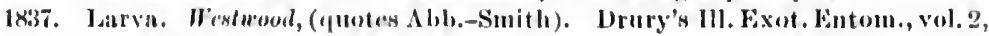
1. 15.

1811. Larvi, pupa. Hhrris. Ins. Inj. Vegetat., 1st edit., p. 285.

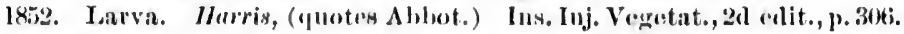

16ïl. Iatrva, (eol'd ligrs.) Vimmons. Nat. llist. N. York, vol.n, p. 232, pl.39.

1658. Isura. Juncun. Natural. Lilurary, vol.2s, p. 1:4.

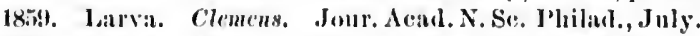

1862. Larva. Morris. Synop. I,p. N. Amer.,p.2e1.

186:. Iarva. Ilarris. Ins. Inj. Fegetat., Flint's edit.,p. Baci.

1869. I.ifo history, (figs.) Liminer. 23l IRept. V. York State Cah. N. IIist., p. 137.

1873. Lifo history, (ligs.) Ril'y. Sth liept. Missmuri, p. 127.

1873. Figg, larra, pupa, (fign.) I'uchurd, (quoten Riley.) Amer. Naturalist, vol. $7, p, 175$.

18Ti. Larva, (tig.) l'uchard, (after Riley.) Italf-hours with Insects, p. Kt.

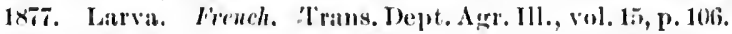

187א. Iaura, (brief.) C. E. 'Vorthington. Cauat. Kutom., vol. 10, p. 1t.

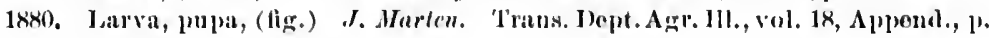
127.

1880. Larrat, pupa, (tig.) D. Coquillett. Trius. Iept. Agr. Ill., vol. 18. $\Lambda_{1}$ pencl., p. 16is.

1885. Jarva. C. T. Goodhue. Canald, Entom., vol, 17, p. 243.

Foon Prant. Ouerens.

Hemileuca nevadensis. Stretch.

1875. Jarva. Hy. Etwarls. l'roc. C'al. Acal. Sce, A pril.

1888. Latrva. G. H. Halst. Lintom. Amer., vol.3, p. 1911.

Food Plant. Sulix.

Hemileuca yavapai.

1880. Larva. Iy. Edwards. Entom, Amer., vol. 3, p. 167.

Foon Pi.ANT. P'rosopis juliflora.

Hemileuca californica. Wright.

1888. Jarva, (bricf.) W. G. Wright. Canad, Entom., vol.20, p.34.

Foon P'taxt. Sulix.

Hyperchiria io. Fabr.

1797. Larva, (col'd fig.) Ablot-s'smith. Lep. Ins. Georgria, p. 97, pl. 49.

1833. Larva, (col'd fir.) Tition P'ale. Lep. Amerieana, pl. f.

18:1. Larva, pupa. IInris. Ins. Inj. Vegetat., 1st edit., p.. 281.

1852. Larva, cocom. Harrik. Ins. Inj. Vegretat., 2al edit., p.303.

18in. Jarvil. Emmons. Nat. Ilist. N. Yonk, vol.5, p.2:2.

18in. Larva. T. Glowr (quotes IIarris.) Trans. N. York State $\Lambda$ err. Soc., p. 81.

18̈̈6. Larva. Fitch. Bil Rept. Ins. N. York, p.379.

1858. Larva. Fileh. 5th liept. Ins. N. York, p.832.

1858. Larva. Duncen. Natural. Liluary, vol.22, p. 157.

1859. Larrat. I'. II. Gasst. Letters from Alabamia, p. 281.

1859. Latra. Clemens. Jonr. Acarl. X. Sc. Philad.,July.

1862. Larva. Morvis. Synop. Lep. N. Amer., p. 220.

1869. Larva, cocoon, (figs.) Harris. Ins. Inj. Vegetat., Flint's ecui., p. 393.

186!. Larva, (fig.) Jar'gre. I.ife N. Amer. Ins., p.124.

186!. Larva, Bethun'. Canal. Entom., vol.2, p.19.

1869. Larva. Harria. Entom. Corres., p. 205.

1870. Larval stagres. Lintucr. 24th Rept. N. York Stato Cal., N. IIist., p. 146.

1873. Ligg. likley. Cauad. Fintom., vol.5, p. 109.

1873. Lifo history, (figs.) Riley. 5th Missouri Rept., p. 133.

1873. Larva, (fig.) Puckard, ('Đnotes Riley.) Amer. Naturiliat, vol.7, p. 477.

187.t. Larva, (fig.) Sutuders. Canal. Entom., vol, 6, p. 227. 


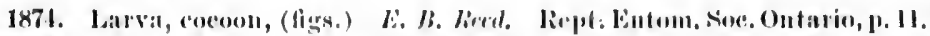

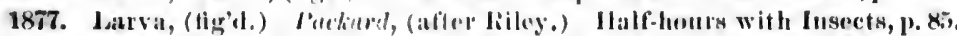

1874. Larva, (tig.) Saumlers. Rept. Entom. Soc. Ontario, p. 75.

1879. Lanva, pupa, (figs.) Mrs. Ballard. Inseet Lives, p.87.

1880. Larva, (fig.) J. Martun. 'Trans. Dept. Agr. Ill., vol. 18, Apponil., p. 127.

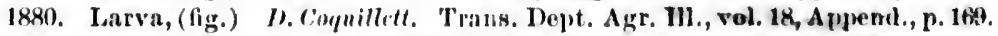

1881. Larva, (fig.) P'ackard, (after Riley.) Ins. Inj. Ferest 'Ireen, p. 111.

1883. Lifo history, brief, (tigs.) Snnmlers. Ins. Inj. Fruits, p.210.

1885. Figg, linra, copoon. Fermald. Kingsley's Stand. Nat. Hist., vol. 2, p. 45 \%.

1888. Larva, pupa, (fig.) A. R. Goofr. 18th Rept. Entom. Soc. Ontario, IIp. 73,74 .

Foon Plodxs. Various trees and shrulis.

Hyperchiria pamina. Neum.

1888. Larval stages. Iy. Éduardx, Eintoun. Aner., vol. 4, p. 62.

Coloradia pandora. Blako.

1888. Egg. Hy. Ellwarls. Wutum. Amer., vol. I, 1. fi.

Eacies imperialis. Drury.

1797. Iarva, (col'd fig.) Abbot-Smith. I,ep. Ins. Geolgia, p. 109, pl. 5i.

1837. Larva, pupa. Hestuool, (quotes Alb.-Sur.) Drury's Ill. lixot. Entom., vol. 1, p. 17.

18I1. Larva, pupa. IItris. Ins. Inj. Vogetat., 1st edit., p. 290.

18\%2. Iarval stages. Haris. ins. Inj. Vegetat., 2dl edit., p. 308.

1858. Jarva, (cot'i fig.) Jukcun. Natural. Libray, vol.22, p.160.

1858. Larva,(brief.) Jitch. łth Kept. Ius. N. York, P. 742.

1859. Larva, pupa, (liricf.) I'. II. Goss', (quotes Abb.-Sui.) Letters from Alaloama, p. 17:2.

1859. Larva, puph. Cl'mens. Jour. Acad. N. Se. Philad., July.

1862. Larva, (fig.) Havix. Ins. Inj. Vegetat., Flint's elit., p.40.4.

1862. Lapra, p"la. Morris. Synop. Lep. N. Amer., p. 230.

1870. Egg, larval stages. Linturr. 2-1th Rept. N. York State Cab. N. Hist., p. 150.

187t. Lavia. Geutry. Camal. Eutom., vol.6, p. 87.

1877. Larva. Frinch. 'Trans. Dept. Agr. Ill., vol.15, p. 186.

1880. Larva. .J. Mart'u. 'Trans. Jept. Agr. Ill., vol. 18, Append., p. 121.

1881. Larvi. I'uckurl, (quotes Harris.) Ins. Inj. Forest 'Trees, p. $20 \%$.

Food Prasts. Cirya, Juylans, ete.

Citheronia regalis. Falsr.

1797. Larva, (col'l fig.) Albot-Swith, (as Plı.regia.) Lep. Ins. Georgia, p. 121, p). 61 .

1833. Larva, (col'd fig.) Titian Peale. Lep. Americana, pl.5.

1811. Lalva, pupa. IIarris. Ins. Iuj. Vegetat., 1st edit., p. 287.

18.2. Larval stages. Hawis. Ins. Inj. Vegetat., 2d edit., p. 309.

1857. Larva. Fitch. 3d Rept. Ins. N. York, p. 456.

1858. Larva, (col'd fig.) Inuean. Nitural.Library, vol. 22, 1, 162.

1859. I,arva. Clemens. Jour. Aead. N. Sc. Plilad., July.

1862. F.gg, larva, pupa. Harris. Ins. Inj. Vegetat., Fliut's edit., p. 100.

1862. Larva, pnpa. Morris. Synop. Lep. N. Amer., p. 230.

1869. Larva, pupa. Marris. Entor. Corres., p. 297.

1874. Larva, (fig.) J. G. Wood." Insects abroad, p. 682.

1877. Larva. Wench. 'Trans. Dept. Agr. Ill., vol. 15, p 196.

1880. Lavva. J. Marten. Traus. Dept. Agr. Ill., vol. 1s, Apnend., p. 122.

1880. Larva. D. Coqnillett, (quotes Harris.) Trans. Dept. Agr. 1ll., vol. 18, Append., p. 162.

1881. Larva. I'ackard. Ins. Inj. Forest 'Trees, p. 76.

1884. Larva, pupa, (brief.) I. Hamilton. ('alual. Eutum, vol. 16, p.47.

Food Pisants. Vurions tiees and slirubs. 
Citheronia sepulcralis. Ai, mul li.

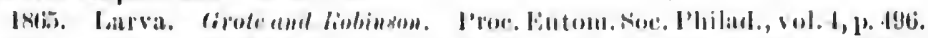

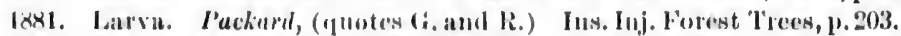

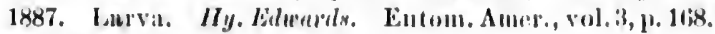

Foon l'lust. l'inus.

Citheronia mexicana. G. and R.

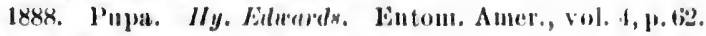

Sphingicampa bicolor. Harris.

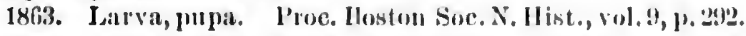

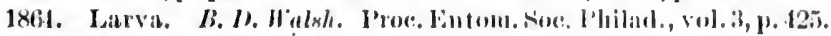

Foon l'Laxt. Cilielitwilit.

Sphingicampa quadrilineata. G. and li.

1888. Pupa. Ity. Edutuly. Entom. Amer., vol. 1, 1.62.

Anisota stigma. lialur.

1797. Larva, (col'd fig.) . Ibhut-Smith. Lap. Ins. (ieorgia, p. 111, pl. 5ti.

1811. Larva, pupa. Htamis, (guotes $\Lambda$ l,b.-sm.) lus. Lu,j. Vugretat., 1st edit., 1. 292.

18:9. Larva. Clemens. Junr. Acal. N. Se. Philadl, July.

1859. Latrva. Fitch. Sth kept. Ins. N. York, p. X:4.

1862. Larva, (brief.) Momis. Synop. Lep. N. Aner., p.231.

1869. Larva, (fig.) Haris. Entom. Corress, p. 298, pl. 11.

1873. Lavva. Riley. 5th Missonri linpt.,p. 126.

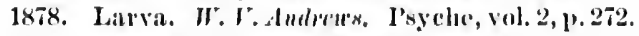

1880. Larva. J. Merten. Trans. Dept. Agr. 111., vol. 18, Appentl., p. 120.

1887. Larva. A. R. Cirole. Canad. Eutom., vol. 19, p. 51.

Foon P'lasts. Varions species of centreus.

Anisota senatoria. Alb.,-Sm.

1797. Larva, (col'd tig.) Abbot-simith. I,p. Ins. (Georeria, p. 113, pl.57.

1837. Latva. Wislwool, ('guotes Al,1,-Sin.) 1)rury's Ill. Exut. Fintom., vol. 2, 1. 2 .

18I1. Larvin, pupa. Harris. Ins. Inj. Yegetat., 1st edit., p. 292.

18:2. Larvi, pinpa. IItrris. Ins. Inj. Vegetat., 2d edit., p.311.

10is. Larva. Fïth. 5th Rept. Ins. N. York, p. 823.

18.9. Larva. Chrmens. Jonr. Aead. N. Se. I'liblat., July.

1862. Latra. Morrix. Synop. Lep. N. Aner., p. 231.

1862. Iarvit, pupa, (fig.) Ilarris. Ins. Inj. Vegetat, Flint's a .,p. 406.

1866. Larva. S. D. Nulsh. I'ract. Eutoun., vol. 2, p. 7.

1869. Larva, pupa, (fig.) Harris. Entom. Corres.,pl.11.

1870. Larva, (lig.) T.Glor'r. Rept. U.S. Dept. Agr., p. 82.

1870. Egrg, larval stages: Lintuer. 2Ith Rept. N. Y. State Cab. Nat. Hist., p. 155.

1877. Larva. French. Trans. Dept. Agr. Ill, vol.15, jo.196.

1880. Jatrat. J. Marten. 'Trans. Dept. Agr. Ill., vol. $18, \Lambda$ ppend., p. 120.

1880. Larva. D. Coquilltt. 'Trans. Dept. Agr. Ill, vol. 18, Appent., p. 161.

1881. Larva. I'tekiarl. Ins. Inj. Forest 'Trees, 11.45.

Foon Plants. Various speeies of Querchs.

Anisota virginiensis, Irury; = pellucida. $\Lambda \mathrm{bh},-\mathrm{Sm}$.

1797. Larva, (col'd tig.) Abbol-Smith. Lep. Ins. Georgia, p. 115, pl. 58.

1811. Larva, pupa. Harris. Ins, Inj. Vegetat., 1st edit., 1. 293.

18氵2. Larva. Hurrie. Ins. Inj. Vegetat., 2d erlit., [1.313.

1851. I arva, pupa, (figs.) Émmons. Nat. Hist. N. York, vol.5, pl.36.

47.59. Larva Fitch. Sth Rept. Ins. N. York, p. 821.

1859. Laria. Chrmens, Jour. Aent. N. Se. Philad., July,

1862. Larva. Harris. Ins. Inj. Vegetat., Flint's edit., p. 407.

1862. Larva. Mtorris. Syuop. Lep. N. Amer., p. 237.

1819. Larva. Harris. Eutom. Corres., 1.298.

1880. Larva. French. 6th Rept. III. Norm. Univers., p. 45. 


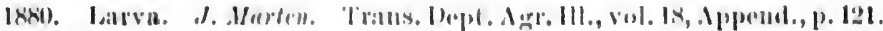

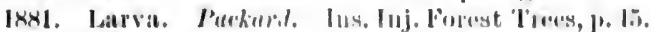

1887. Larva. A. R. Grote. Canal, lintom, vol, 14, 11.52.

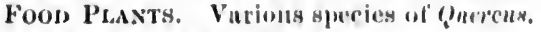

Dryocampa rubicunda. Fulir.

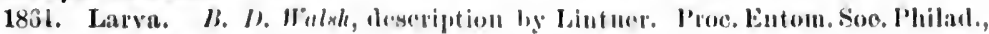
vol. $3, \mathrm{p} .12 \mathrm{t}$.

1870. Larva. Sumbler. ('unaml. Eutum, vol.2, 19.75.

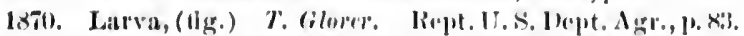

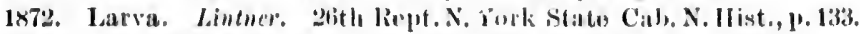

1873. Lifo history, (tiges.) likley. 5th Missomri linpt., p. 1:37.

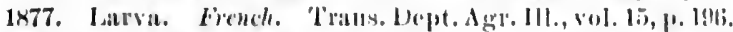

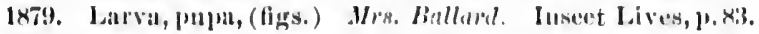

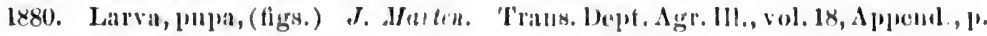
121.

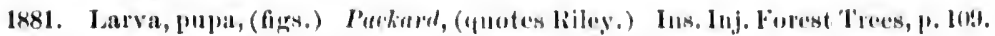

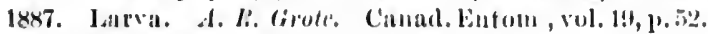

Foon Prants. Ace', rte.

Clisiocampa californica. l'alk.

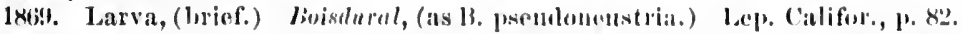

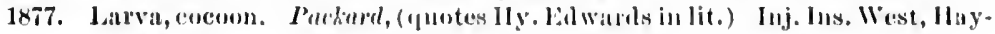
den's Rejit., j. 80T."

1881. Latra, coeoun. Strith. P'apilin, vol, 1, p.lit.

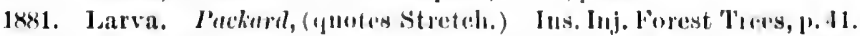

1881. Larva, coeoon. Fachard, (quotes Hy. Eawards in lit.) los. Inj. Forest 'l'iees, 11. 13, *

Foon Peants. Guerens agrifolia, linbus, ete.

* Notw.-lhis description was given by me to Dr. lackald in error as Gastro. pacha californim.-11. li.

Clisiocampa americana. Iasris.

1796. Lifo history, (lirief.) Perli. Proc. Mass, Mgr. Soce.

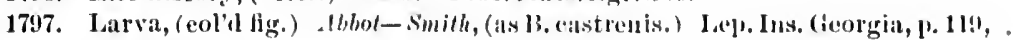
1). 60.

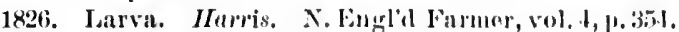

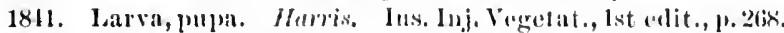

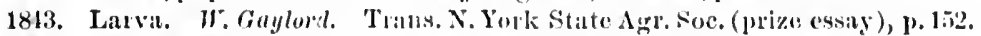

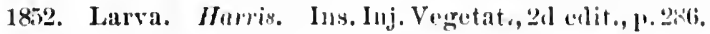

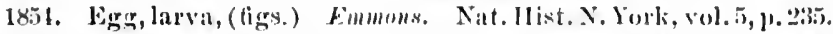

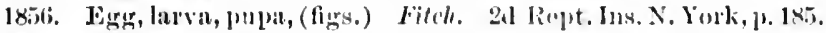

1862. Figg, larva, cocoun, (figy.) Jarris. Ins. Inj. Vegetat., Flint's edit.,p.371.

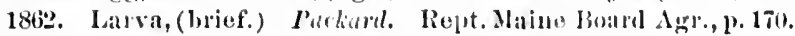

1861. Lava. Jacger, Lifo N. Amer. Inseets, p. 171.

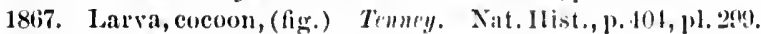

1869. Larva. Boisdural, (as 13. frutetorum.) Lep. Carlifor., P. K2.

1870. Fge, larva. T. Clorer. liept. U.S. Dept. Agr., p. X3.

1871. Ligg, larva, cocoon, (ligs.) líl'y. 3a Missonri Rept., l. 118.

1872. Egg, larva, cocoon, (figry.) Sommlers. Cumal. Eutout, vol.4, p. 134.

1873. Egg, (fig.) Lilly. 5th Missouri Replt., p. 56.

1873. Egg, larva, cocoon, (ligrs.). A. J. Cooh. 12th Agr. Rept. Michigan, p, 131.

1875. Egg, larva, cocoon, pupa, (figs.) Sunders. Rept. Entom. Soc. Ontario, p, 30.

1876. Egg, larva, cocoon, (figs.) G. II. Perlins. Rept. Vermont Board. Agr., p.586.

1877. Egg, Iarva, pupa, (figs.) Peckarl. Iu.j. Ins. West, Haylen's Rept., p. 793.

18'i7. Kgg, larva. French. Traus. Dept. Agr. Ill, vol.15, p. 197.

1877. Egg, lava, cocoon, (fig.) B. Golt. Rejt. Lintom. Soc. Ontario, p. 41.

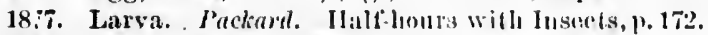

1873. Egg. Saunder8. C'anad. Entom., vol. 10, p.21. 
1879. Figg, larva, cocoon, (tigs.) II. C'utting. N. Hamj., Bonrd Agr.,p. 17.

1880. Egg, larva, cocoon, (figs.) J. Murten. 'Trans. Dept. Agr. Ill., vol. 18, Append., p. 122.

1880. Egg, larva, cocoon, (figrs.) D. Coquillett. Trans. Dept. Agr. Ill, vol. 18, Append., p. 15i.

1883. Egg, larva, cocoon, (figs.) Suunders. Ins. Inj. Fruits, p. 48.

1887. Egg, larva, puja, (ligs.) J. Fletcher. Rept. Entom. Dept. A grr. Can., p. 24.

1888. Egg, larva, eoeoon, (figs.) J. Fleteher. Rept. Bot. Entom. Dept. Agr. Ciunala, p. 24.

1889. Egg, larva, coeoon, (figs.) J. Fleteher. Canad. Eutem., vol. 21, p. 74.

Food Plants. Various trees and shrulss.

Clisiocampa constricta. Stretch.

1874. Larva, cocoon, pupa. Iy. Educards. Proc. Cal. Acal. Se., September.

1881. Larva, cocoon. Stretch. Fapilio, vol. p. 65.

1881. Larva. Packurd, (quotes IIy. Edwards.) Ins. Inj. Forest Treen, p. 41.

Food Phast. Querchs sonomensis.

Clisiocampa erosa. Stretch.

1881. Cocoon. Stretch, (quotes Hy. Edwards in lit.) Papilio, vol. 1, p. 67.

Clisiocampa disstria. Hübn. =Sylvatica. IIarris.

1797. Larva, (col'd fig.) Albot-Smith, (as G. nenstria.) Lep. Ins. (Georgia, p. 117 , pl. 59.

1811. Larva, pupa. Murris. Ins. Inj. Vegetat., 1st edit., 1. 271.

1844. Larva. Harris. N. Eng'd Farmer, vol, 22, p. $41 \%$.

1852. Larva, cocoon. IIarris. Ins. Inj. Vegetat., 2d edit., p. 291.

185. Larva, cocoon, (figs.) Emmons, (as 13. neustria.) Nat. llist. N. York, vol. $5, \mathrm{p}, 240$.

1856. Larva. Fitch. 2d Rept. Ins. N. York, p. 198.

1858. Larval stages. Fitch. 5th Rept. Ins. N. York, p. 820.

1862. Larva, (figs.) Harris. Ins. Inj. Vegetat., Flint's edlit., p. 375.

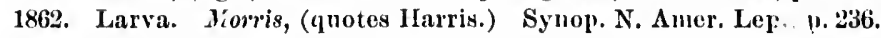

1864. Larva. Jueger. Life N. Amer. Ins., p. 173.

1869. Larva. Fílitis: Entom. Corres., p. 292.

1871. Egg, iarva, (fir'tl.) Riley. 3d Missonri Rept., p. 121.

1874. Lal va, (fig.) .r. G. Wood. Insects Ahroal, p. 680.

ióco. Lanva, (fig.) Saunders, Rept. Entom. Soc. Ontario, p. 30.

1877. Lar"a. $r$ Gott. Rept. Entom. Soc. Ontario, p. 41.

1877. Egg, larva, (figs.) I'ackard. Inj. Ins. West, Haycien's I.ept., p. 794

1878. Egg, larva, (figs.) (i. H. Perkins. Rept. Vermont Board Agr., p. 258.

1878. Egg, larva, (figs.) Sannders. Reat. Entem. Soc. Ontario, p. 28.

1879. Egg, larva, (figs.) H. Cuttin!. N. Hamp. Board Agr., p. 20.

1878. Egg. Saunders. Canad. Entom., vol. 10, p. 21.

1880. La' l, (fig.) J.Marten, (quotes Fi ch.) Trans. Dept. Agr. Ill., vol. 18, A ppond., p. 124.

1880. Iarva. French. 6th Rept.Ill. Norm. Univ., p.44.

1881. Egg, larva, (figs.) Packurd, (quotes Fitch.) Ins. Inj. Forest Trees, p. ‘0.

1883. Egg, larva, cocoon, (figs.) Saumers. Ins. Inj. Fruits, p. 53.

1887. Egry, harva, (figs.) J. Fletcher. Rept. Entom. Dept. Agrr. Caur., i., 21.

1888. Egg, larva, cocoon. J. Fletcher. Rept. liot. Ent. Dept. Agr. Cunada, p. 21.

Food.Plants. Pyrug, Prinute, Querens, etc.

Clisiocampa fragilis. Stretch.

1888. Cocoon. Hy. Edluards. Entom. Amer., vol. 4, p. 62.

Gastropacha americana. Harris.

1797. Larva, (cel'd fig.) Abbot-Smith, (as B. ilicifolia.) Lep. Ins. Ten"*ia, p. 101, pil. 51 .

1811. 1.arva, pupa. Harris. Ins. Inj. Vegetat., 1st erit., p. 273.

18i,2. Ianrva. IIarris. Ins. Inj. Vegetat., 2d edit., p. 293. 


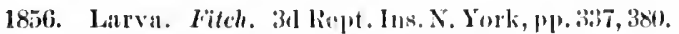

186\%. Larva. Harris. Ins. Inj. Veretat., Flint's edit., p. 37\%.

1869. Larva. Harris. Lintom, Corres., p.2!2.

1879. Larva. Lintmer. 2uth Rept. N. York Stato C'al. N. Hist., 1. 15t.

187t. Egg. H. H. Lyman. Canad. Eostom., vol. 6, p. lis.

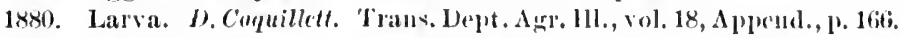

1863. Egg, larva, cocoon. Saunders. Lus. Inj. Frutits, p. 87.

Food Plants. Pyrus, Froximes, Guereus.

Tolype velleda. Stoll.

1797. Larva, (eol't lig.) .lbbot-Smith. Lep. Ins. Georgia, p. 103, pl. 52.

1811. Larva, prpa. Ilarris. Jus. 1nj. Vegetat., 1st edit.,p. 27 I.

1818. Larva. Miss Itix. Silliman's four. of se. and $A$ rt, vol, 19, p. 69.

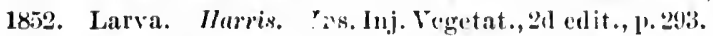

18,6. Larva. Fitch. 3il liept. Ins. N. York, p.333.

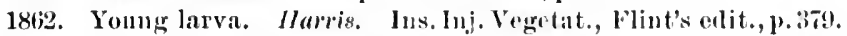

1869. Larva. Harris. Jintom. Corres., 1.2.213.

1872. Larva. Liutner. 26th Rept. N. York State ('ill. N. Ilist., p. 134.

1877. Larva. French. Trans. Dept. A rro 1ll., vol. 15, p. 1:47.

1880. Larva. I). Coquillat, ('1uotes Lintuer.) 'Tlans. Dept. Agr. Ill., vol, 18, Append., p. itit;.

1883. Larva. Eduarls-Elliot. Pappilio, vol.3, p. 1330.

1883. Egrg. Hy. Siluads. I'apilio, vol. 3, p. 189.

1883. Larva, cocoon, (ligs.s.) Sulunders. Ins. Inj. Fruits, p. 89.

Food Piants. P'opulus, Prumus, te.

Tolype laricis. Fitch.

185̄6. Life history. Ittch. 2d. linpt. Ins. N. York, n. 264.

1881. Larva, cocoon. Pacherl, (ynotes Fitch.) Ins. Inj. Forest Trees, p. 251.

1883. Cocoon, (brief.) II. R. Gillbert. I'apilio, vol.3, p.25.

1883. Larva. Eidwards-Elliot. L'apilio, vol. 3, p. 131.

Food) Plast. Larix americana.

\section{Sul.-fiun. Cossida.}

Cossus centerensis. Lintuer.

1879. Lifo history. Jas. S. Bailey. Canat'. Entom., vol.11, p. 1.

1880. Larva. D. Coquillet, ('putes Bailey.) Trans. Dept. $\Lambda$ gr. Ill., vol. 18, $\Lambda$ ppend., p. 151.

1881. Larva, pupa. Paditrol, (quotes Bailey.) Ins. Inj. Forest Trees, 1. 120.

1883. Pupa, (brief.) J. Fletch'r. Cantul. Entom., vol. 15, p. 203.

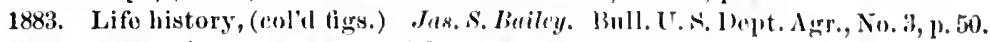

1885. Pupal, (figs.) Lintur?. 21 Rept. State Entom. N. York, 1.216.

Foon Pixter. Populus tremuloides.

Cossus alni. Kellieott. (Now sp.?)

1885. Larval. 's. Kellicott. Entom. Aner., vol. 1, p. 17 is.

Prionoxystus robiniæ. Peek.

1821. Life history, (hrief,) (figs.) P'eck. Mass. Agr. liepos. and Jour, vol.5, p. 67.

1841. Larva, pupa. 'Tarris. Ins. Inj. Vegetat., 1st whit. 1. 2:Hi.

1852. Life history. Iterris. Ins. Inj. Vegetat., 2d edit., p. 316.

1858. Life history. Fitch. 5th Rept. Ins. N. York, p. "ij'.

2.1.

1862. Larva, pupa, (figs.) Haris. Ins. Inj. Vegetat., Flint's edit., p. 411

* 187i. Larva. Pachuerd. IIalf-lionrs with Insects, p. 259.

1877. Larva. Fe'nch. Amus. Dept. Agr. Ill., vol. 15, p. 198.

1880. Larva. I). Coquillett. Trans. Dept. $\Lambda$ gr. Ill., vel. 18, Append., p. 152.

1881. Eigr larva, pupa. I'ackurl. Ins. Inj. Forest Trees, p. $\mathrm{i}$.

i881. Larva, pupa, (brief.) I. Rellicott. Bull. Buflalo Soc. N. Itist., vol. 4, p. 30.

Feess in trunks of Cucreus, Robinia, Populus, Salix, ctc. 
Zeuzera æsculi. LımIn.

1829. Lanvi, pupa. Strphorus. 1llust. Brit. Eutom., Inawkes, vol. 2, p. 8.

1829. Latria, (fig.) A. Mattheter. Mag. Nat, llist., vol, 2, p. b6.

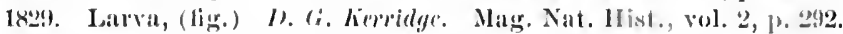

1837. Larvi, pupa. Loller. Ins. Inj. Vegetat., London's colit., p. 208.

16\%9. Larva, (col'd fir.) ¿ntis. Brit. Lintom., vol. 16, 11. 722.

1810. Larva, pupal, (col'd ligs.) lintzeburg. J)ie Forst-Insecten, vol. 2, p. 88, 11. 3,1.

1810. Larva, (briet.) Westroot. Ints. Mool. Class. Ins., vol. 2, p. 378.

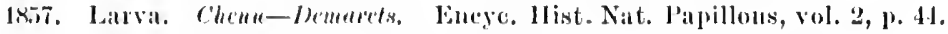

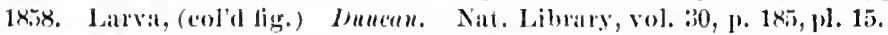

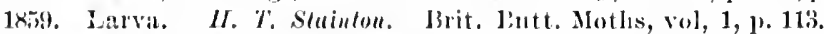

I859. Larva, (col'd tig.) Ilumphreys. (ienera Brit. Motlis, vol. 1, p. 15, pl.4.

1869. Larva. Nemman. Lititish Moths, p. 18.

1872. Larva, (lig.) J. G. Hoorl. Insects at Home, ]. 428.

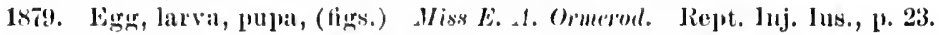

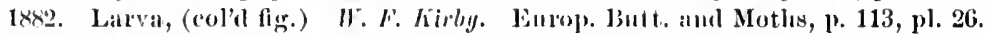

Fevis in trunk and branches of Querens, sollix, Populus, dte.

Cossula magnifica. Bailey.

188:2. Lite history, (hricf.) J. s. Burily. Papitio, vol. 2, 1.91.

1883. I'npa, (col'd fig.) .J. S. Bailey. BuIl. Dept. Agr., No.3, pl.2.

Food Plast, Guerens rirens.

Hepialus argenteomacuiatus. IIarris.

Sub-ftul. Hepialida.

1810. Egrg, (brief.) I. II. Cosse. Canad. Naturalist, j. 248.

1888. Larva, notes on. I). S. Fellicolt. Entom. Amar., vol. 4, l. 153.

1889. Life history. D. S. Liellicott. lisect Life, vol. 1, p. 200.

Bombycia improvisa. Hy. Eilwards.

NOCTUA.

1873. Pupr. Hy. Liduarls. Proc. Cal. Acad. Se., November.

Pseudothyatira cymatophoroides. Ginen., (ind viur.)

1863. Larva. A. R. (ivote, ('puotes Cutler in lit.) Proe, Entom. Soc. Philad., vol. $2, \mathrm{p} .131$.

1883. Larvit. R. Thuxter. Papilio, vol.3, p.10.

lood P'Lant. Guerens.

Habrosyne scripta. Ciosse.

1883. Eggr, latva. R. Thuxtes. Papilio, vol.3, p. 10.

Food Plant. Liubus.

Platycerura furcilla. Packard.

1872. Larva, (fig.) Lintuer. 26th Rept. N. York State Cab. N. Hist., p. 132.

1874. Lanva, (figr.) P'acliarl, (quotes liutner.) Amer. Naturalist, vol.8, p. 691.

1880. Larva. I). Coquilletl, (fnotes Lintner.) 'Traus. Dept. Agr. 1ll., vol. 18, Append., p. 168.

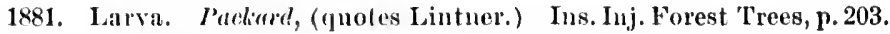

18:" Larval, cocoon. R. Thaxter. Papilio, vol. 3, p. 11.

1X,. . Larva. Fernald. Kingsley's Stand. N. Ilist., vol. 2, p. 453.

Food I'Laxts. Pinus, Tilia.

Charadra propinquilinea. Grote.

1881. Larra. L. II. Goodell. Papilio, vol.1, p. 15.

1883. Latva. R. Thaxter. Papilio, vol.3, p. 12.

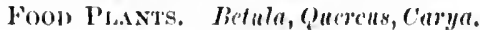

Charadra deridens. (incell.

1870. Lanva. Saunders. Canad. Eutom., vol. 2, p. 11 i.

1872. Larva, coeoon. Lintuer. 26th Rept. N. York State Cab. N. Hist., p. 157.

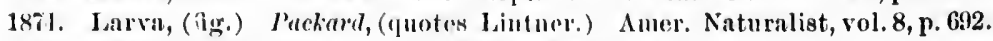

1883. Jarval stages, cocom. R. Thuxter. L'apilio, vol.3, p. 11.

Food Plant, Guerent. 
Raphia frater. lirote.

1883. Latria. Li. Thexter. lappilio, vol, 3, p. 13.

Apatela grisea. Walliw.

1883, Latrit. Liduterds-Eilliot. Ditpilio, wol. 3, p. 131.

Foon Plaxts. Clmus.

Apatela falcula. (irote.

1881. Larvat. D. Counillutt. Papilio, vol. 1., p.ti.

Fuon l'inds. Corylus.

Apatela occidentalis. (i. and li.

1869\%) Latrit. Herris. Entom. Contess, 1, 311.

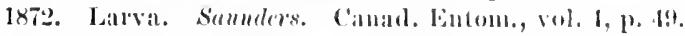

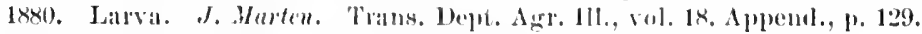

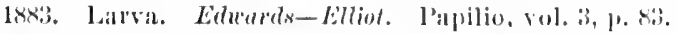

18x:3. Eggr, lanva, pupa, coeoon. Sentulers. Jus. Inj. Finits, p. 16it.

Food l'Laxt. rerelshs.

Apatela lohelix. (incu.

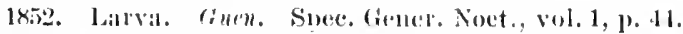

1881. Latrat. D. Coquilletl. l'apilio, vol. 1. p. li.

1886. Larva, Fench. Canad. Fintom, vol. 18, p.118.

Fool, liast. encrens.

Apatela morula. (i. ant $\mathrm{k}$.

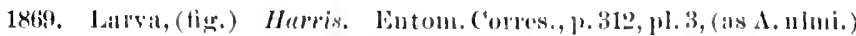

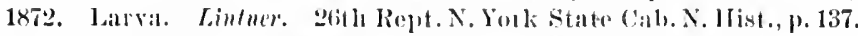

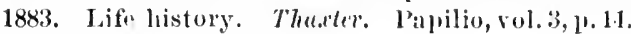

Foon I'last, U hmos.

Apatela vulpina. (irote.

1883. Larra. Thuxter. Papilio, vol.3,p.1J.

Food Plastrs. Betulu, Populus.

Apatela radcliffei. IIarvey.

1878. Latra, cocoon, pupa. Thext'r. Psyehe, vol, 2, p. 121.

Foon l'saxt, Promus serutinu.

Apatela spinigera. (incill.

1878. Larva. Tharter. J'syche, vol. 2, 1. 121.

Foon Praxts. liubus, Betulu.

Apatela betulæ. Riley.

1884. Lamva, pupa. Riley. Bull. Brooklyn Eutom. Soe, vol. 7, p. 2.

Hood least. befllet nigrel.

Apatela spinea. (ir.

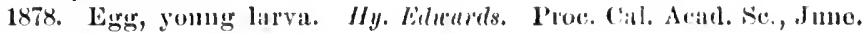

Foon I'laxt. Lupiuns.

Apatela lepusculina, (4urn.

1875. Latrva. Hy. Eiltertls. Proc. Cal. Aeat. Se., April.

Foon I'LANT, P'omilus.

Apatela populi. liiley.

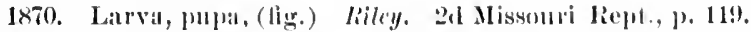

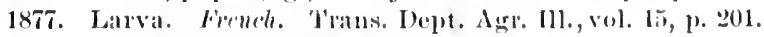

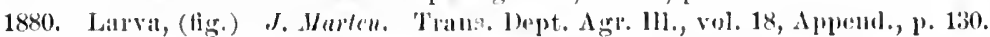

1881. Lavia, pupal, (tig.) Inckintel, (after Riley.) Ins. Inj. Forest Trees, p. 116.

1882. Larril, (fig.) Sumuters. Canad. Entom., vol.11, p. 221.

18x:. Lalria, (lig.) Sammiers. Rept. Entont. Soe. Ontario, p. 21.

Foon Plast, J'opulus.

Notw.-The two speeies (undonhtedly distinct), A. lemesculine and A. populi, have

57.

. 692. heen confounder, and some of tho deseriptions of atrly stages of the lat ter have heen griven under the former nime. A. lepusculina appears to be contined to the Pacilic coast.-II. E.

BULL. 35-6 
Apatela felina. (irote.

1887. Lamal stages, pupla. Fronch. Canad. Lintom., vol. 1!1, p.19.

Foon I'taxt. Sulix.

Apatela americana. II:tris.

1797. Larvit, (col'd tig.) Abbot-Smith, (as $\Lambda$. aceris.) Lep. Ins. Geornia,p. 185, 1). 93.

1811. Larrit. IIaris. Ins. Inj. Vegetat., 1st edit., p. 317.

18i2. Larva. Harris. Ins. Inj. Vegetat., 2d edit.,p.33i.

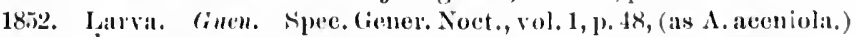

186:. Larva, puja, (tigs.) Huris. Ins. Inj. Vegetat., Fint's edit., 1. 136.

1869. Larva, (tig.) Harris. Entom. Corres., p. 311, pl.111.

1872. Larva, Linture. 266th liopt. N. York State Call, N. Ilist., pp. 1355, 157.

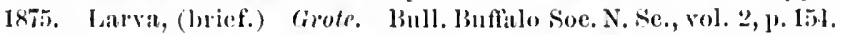

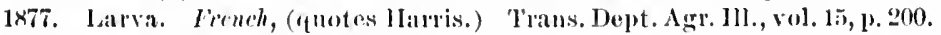

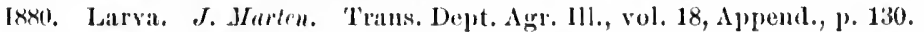

1881. Latria. 1), Coqnillett. I'apilio, vol. 1, l. 6.

1881. Iarva. I'ackionl. Ins. Inj. Forest Trees, p. 111.

Foon l'lave. Acror.

Apatela hastulifera. Mls.-Sm.

1797. Lalva, (col'd lig.) Albbi-Smith. Lep. Ins. Georgia, p. 183, pl. 92.

18.2. Larvi. Gurn. Spee. (iener. Noct., vol. 1, p. 17.

18i2. Lanva, cocoon. Lintucr. 26th Rept. N. York State Cab. N. Llist., p. 158. foon l'aAsts. Esculus, Acer.

Apatela rubricoma. (inen.

18.5. Latra. Gucu. Spee. Gencr. Noet., vol. 1, 1. 48.

1880. Larva Fechch. 6th Rept. Ill. Stato Nomm. I'niver, p. 45.

1880. Larva. J. Marten. Trans. Dept. Agr. Ill., vol. 18, Append., p. 132.

Foon Plast, Celtis erersifolia.

Apatela luteicoma. G. and K.

1883. Larva. Thaxter. Papilio, vol. 3, p. 16.

Foon Plants. Tilin, Acer, Freximus.

Apatela clarescens. (inen.

1869. Larva, (fig.) Ifarris. Entom. Corres., p. 313, pl. I, (as $\mathrm{A}$. pruni.)

Foop l'laxt. Prumus.

Apatela brumosa. Ginen.

1852. Larva, (col'd fig.) (inen, (after Ablot, MS.) Spec. Gener. Noct., vol. 1, l. 52, pl. 2.

1881. Larva. D. Coquillett. Papilio, vol. 1, p. 56.

1883 Larva, pupia. Samuders. Ins. Inj. Fruit, p.313.

1883. Larva. Sumders. Rept. Entoni. Soe. Ontario, p. 12.

Food liants. Corylus, Prunus.

Apatela noctivaga. Grote.

1883. Lifo history. Thaxter. Papilio, vol.3, p. 15.

Foon Plant. ropulus.

Apatela superans. Ginen.

1871. Lama. Lebaron. 2al Illinois Rept., p.51.

1880. Lalva. J. Marten. 'T'raus. Dept. Agr. Ill., vol. 18, Append., p. 131.

1881. Larva. 1). Coquillett. Papilio, vol. 1, ]1.6.

1883. Eggr, larva, (fig.) soumders. Ins. Inj. Fruits, p. 166.

Foon Plast. Prumus.

Apatela afflicta. Grote.

1883. Larva. Thaxter. Papilio, vol. 3, p.17.

Foon litant. Quereus.

Apatela lanamelis. Ginen.

1852. Larva, (eol'd lig.) Gu'n, (after Abb., as.) Spee. (iener. Noct., vol. 1, 1. 52, pl. 2. 


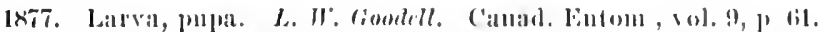

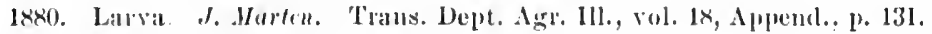

Four) P'Lsiser. C'astunta.

Apatela lithospila. Grote.

1883. Lillva. Edwards-Elliot. Paprilio, vol. 3, 1. 1:32.

Foon Plant. C'urya ullet.

Apatela oblinita. Al,,$-S i n$.

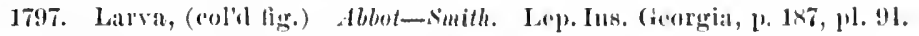

1852. Larva. Gime. Spece (imer. Noet., vol. 1, 1. 19.

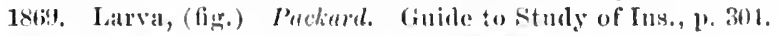

1871. Latra, pupa, (fig.) Riley. 31 Missmuli Rept., 1. T0.

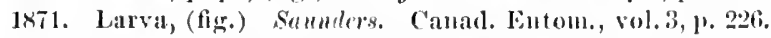

1872. Larva. Lintuer. 26th Rept. N. York State Cab. N. Mist., 1. 159.

1877. Larva. French, (fuotes Rilry.) Trans. Dept. Agr. Ill., vol. 15), P. 201.

1878. Lama, pupa, (figs.) (i. M. levlius, (aftor Riley.) Eth Rept. Vormont Board Arr., ए, 275.

1878. Lavia. L. W. Goodell. •Canad. Enton., vol. 10, p. 6it.

1880. Lauva, foeoon, (ligs.) J. Marten. Trans. Dept. Agr. Ill., vol. 18, Append, p. 131.

1880. Larva, cocoou, (fig.) 1). Coquillett. 'Trans. Depit. Aör. Ill., vol. 18, Appenul., - 1. 170 .

1883. Larva, cocoon, pupa, (ligs.) S. A. Forbes. Trans. Wisconsin Arrr. Soc., vol. 21, 1. 7.

1883. Larva, cocoon, (lig.) Samullers. Ins. Inj. Finits, p.,325.

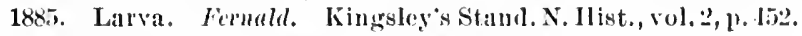

Foov Plaxts. I'olygomum, l'ench, Aspuragus, etc.

Arsiloi.che albovenosa. Grote. =henrici. (ir.

1877. Larva. Tharter. Psyche, vol. 1, p. 188.

1880. Iarva. I). Coqnillett. Canad. Eitom., vol. 12, p. t5.

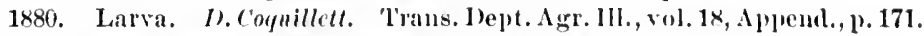

1888. Latrial stages. II!. Edeards. Entour, Amer., vol, 3, p. 171.

Foon Plants. Polygonum, etc.

Harrissimemna trisignata. Walk.

1869. Larva, pupa,(fig.) Hovis. Eutom. Corres, Pl. 111, 17.1.

1869. Larva, (lig.) Picliard. (inide to Study of Ins., p. 301.

1886. Larva. C.F. Goothue. Canad. Entom., vol. 18, 1. 58 .

Food Plant. Syringa.

Agrotis c. nigrum. Limn.

1829. I,arva, pupa. Stpltens. Illus. Brit. Eutom., Hanst., vol. 2, p. 136.

1859. Larva. II. T. Stainton. Brit. Butt., Moths, rol. 1, 1. 231.

1877. Larva. C. Thomas. 'Trans. Dept. Agr. Ill., vol. 15, p. 89.

1877. Larva. French. Trans. Dept. Agr. Ill., vol. 15, p. 202.

1880. Larva. J. Marten. Trams. Dept. Agr. Ill., vol. 18, Append., 1. 132.

1880. Larva. 1). Coquilletl. 'Iraus. Dept. $\Lambda$ gr. Ill., vol. 18, Ajpend., 1. 181.

1882. Larva. T. F. Kirby. Europ. Bntt. and Motlis, 1) 192.

1883. Larva. Eilunte-Elliot. Papilio, vol.3, p. 133.

Agrotis baja. S. V.

1829. Larva. Stephens. Fllus. Brit. Futom., IIanst., vol. 2, p. 131.

1859. Larva. II. T. Slainton. Brit. Butt., Moths, vol. 1, 1. 237.

1882. Larva. W. F. Kirby. Eurol.. 13ntt. and Moths, 1) 191.

Agrotis fennica. Tanscl.

1881. Larva. J. Fleteher. Canad. Entom., vol. 16, p. 204.

Agrotis subgothica. Haw.

1869. Larva, (fig.) liiley. 1st Missonti linpt., p. K1.

1877. Larva. C. Thomes. 'T'ans. 1)ept. Agr. Ill., vil. 15, p. 89.

1877. Larva. French. 'Trßıs. Dept, Agr. Ill., vol. 15, 1. 201. 


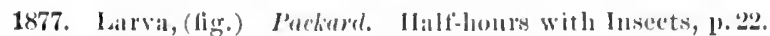

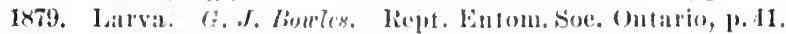

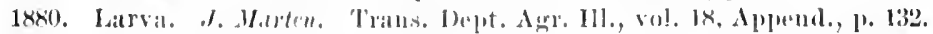

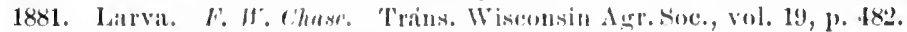

1883. Latua, (brief") sammlors. Ins. Inj. Frnits, 1. 328.

Agrotis tricosa. Lintuel'.

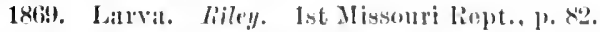

1880. Larva. I. Marten. 'Trans. Dept. Airr. Ill., vol. 18, Append., p. 138.

Agrotis herilis. Grote.

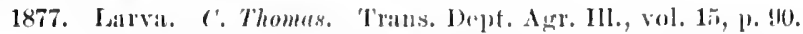

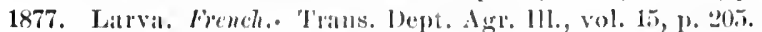

1880. Larva. J. Marten. 'Trans. Dapt. Agr. Ill., vol. 18, Append., p. 133.

Agrotis plecta. Liun.

1899. Lavia. Stephens. Illus. Brit. Entom., Itaust., vol. 2, 1. 137.

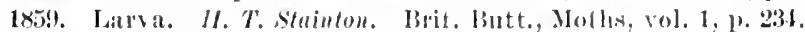

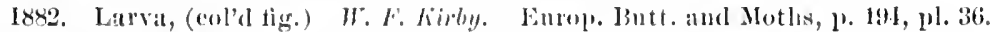

Agrotis cupida. Cirolo.

1880. Larvat. J. Maten. Trans. Dept. Agr. Ill., vol. 1i, Append., 1. 136.

Agrotis clandestina. Jurris.

18.1. Larva. Harris, (ynotes Melsheimer in lit.) Ins. Inj. Vegetat., 1st edit., p. 325.

1869. Larra, (fig.) Riliy. 1st Missouri Rept., p. 79.

1871. Lanva. Saunders. Canad. Entomi, vol.3, p. 35.

1876. G. II. Perhins. 2d Rept. Vermont Loard Agr., 1. 597.

1877. Larva. C.Thomes. 'T'rans. Deple. Agr. Ill., .15, 1. 95.

1877. Larva. French, (quotes Riley.) 'Trans. Jept. Agrr. Ill., vol. 15, p.213.

1877. Lalva. I'ackarl. Inj. Ins. West, llayden's Rept., n. 717.

1877. Larva, (ligg.) Peckard. IIalf-lonrs witlı Inseets, p.23.

1879. Larva, (figr.) (H..J. Bonles, (funtes Riley.) Rept. Entom. Soc. Ontario, p. 14.

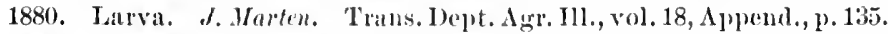

1883. Larva, (brief), (lig.) Sunulers. Ins. Inj. Fruits, p. 108.

1884. Larva, (lnief), (fig.) Rilly. Rept. Eutom. (T. S. Dept. Agr., 1. 293, pl. 2, tigr. 1 .

1885. Larva, (fig.) Linluer. 4th Rept. N. York St'io Agr. Soc., p. 57.

1888. Larva, (lig.) Lintuer. Bull. N. Y. State Musenm, No. 6, 1. 4.

Agrotis messoria. IIarris.

1877. Larva. C.Thomas. 'Trans. Dept. Agr. Ill., vol. 15, p. 92.

1877. Larva, (tig.) Fremeh: 'Trans. Dept. $\Lambda$ grx. Ill., vol. 15, p. 209.

1879. Jarva, (fig.) G.J.boules. Rept. Entom. Soe. Ontario, p.42.

1880. Larva, (fig.) J. Murten. Trans. Dept. Agrr. Ill., vol. 15, Append., p. 13.1.

1881. Larva, (fig.) F. Ir. Chase. Trans. Wiscousin Agr. Soc, vol. 19. p. 484.

1883. Larva, (lig.) kiley. Amer. Natmalist, w. 17, p. 422.

1884. Larva, (fig.) Riley. Rept. Entom. U. S. Dept. Agrr., p. 290, pl, 2, fig. 6.

1886. Life history, (firs.) Riley. Rept. Entom. U.S. Nept. Agr.

1888. Larvi, (fig.) Limtuer. Bull. N. Y. State Musenu, No. 1, p. 13.

Agrotis scandens. Riley.

18:8. Larva, (1igss.) Riley. 1st Missomi Rept., n. 76.

1877. Larva. Freneh, (quotes Riley.) Trans. Dept. Ag1. Ill,, vol. 15, p. 208.

1879. Larva. G. J. Boules, (quotes Riloy.) Rept. Entom. Soc. Ontario, p. 43.

1880. Larva. J. Marton. Trans. Dept. A igr. Ill., vol. 18, Append., p. 133.

1883. Larva, (lig.) Lilcu. Auer. Naturalist, vol. 17, p. 422.

1883. Larva, (fig.) Sammlers. Ins. Inj. Fruits, p. 108.

Agrotis pitychrous. Grote.

1889. Larva. П. Bentenmillev. Futom. Amer., vol. 5, 1. 38.

Agrotis tesselata. IInris.

1863. Larva. Fitch, (as A. maizii.) 9th Rept. Ins, N. York, p. 80.4, 


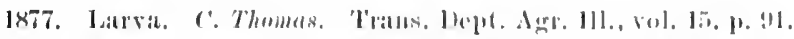

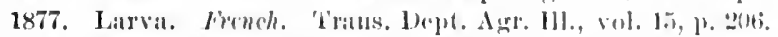

1879. Larva. G. J. Bowle, (quotes Fitel.) Rept. Eutoun. Soc. Ontirin, p. 39.

1879. Larva. IV. A. Burkon. Agr. Ponnsyly, p. 3 f.

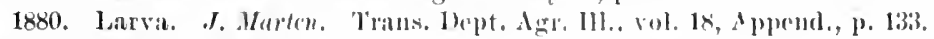

Agrotis declarata. Walker.

1885. Larva. Sunders. Canacl. Entum., vol. 17, p. 325.

Agrotis malefida. (inen.

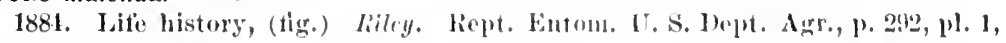
fign. 3.

1888. Larva, (tig.) Lintuer. Bubl. X. Y. State Muselim, No. 1i, 1. 11.

Agrotis cochranii. Riley.

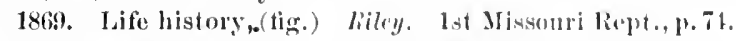

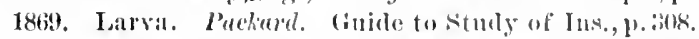

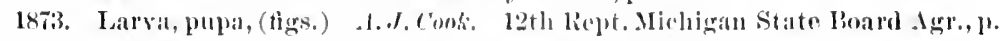
110 .

1877. Larva, (fig.) Pecherd. Italf-hours with Insectis, p.23.

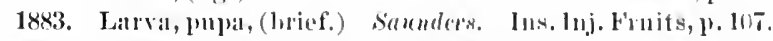

1888. Lallia, (fig.) Bethure. 18th liept. Butem. Soe. Ontario, p. 5.

Agrotis annexa. 'l'r.

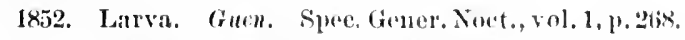

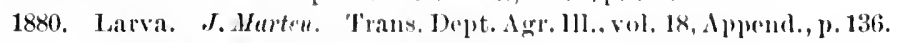

1882. Life history. French. Canarl. Entom., vol. 11, 1.2017.

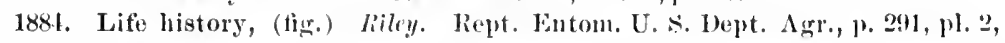
fig. 1.

1885. Larva, pupa, (figs.) Linture. Hlth Rept. N. York State Agr. Soe, p. 62.

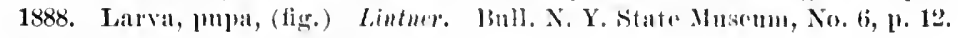

1.1.

Agrotis ypsilon. Rott. = suffusa. S.V.

1829. Larva. Stephens. Illus, Brit. Fintom., 1Iatust., vol.2, p. 149.

1859. Larva. H. T. Stainton. Brit. Butt. and Noths, vol. 1, p.e23.

1869. Larva, (fig.) liley. 1st Missonri Rept., p.80.

1869. Larva, (fig.) I'ackard. Ginide to Stuely of Ins., p. 306.

1877. Larva. C. Thomets. Trans. Dept. Agr. MH., vol. 15, p.93.

1877. Larvi. Trench. Trans. Dept. Agr. IH., vol. 15, p.210.

1877. Larva, (figs.) Packurl, (quotes Riley.) Inj. Ins. West, Iascden's Rept., p. 717.

1879. Larva. G..J. Bomles. Rept. Entom. Sue. Ontario, p. to.

1880. Larva. J. Martett. Traus. Dept. Agr. Ill., vol. 1s, Aplend., p. 131.

1883. Larva, (fig.) Suruders. Ins. Inj. Fruits, p. 320

1884. Egr, larra, (fig.) Liley. Rept. Eittom. U. S. Dept. Agr., p. 291, pl. 2, fig. 2.

1888. Larva, (fig.) Lintucr. Inll. N. Y. State Museum, No. 6, p. 15.

6.

1888. Larva, (fig.) bethune. 18th Rept. Entom. Noc. Ontario, p. 5. .

Agrotis islandica. Standgr.

1873. Larva. I'ackird. In Iayden's Geol. Surv. Terr., p. nab.

Agrotis saucia. Ilübn.

18:59. Larva. H.T. Stuinton. Frit. Bntt. and Moths, vol. 1, p. 22.4.

186\%. Lifo history, (tigs.) Liiley. 1st Missouri liept., [r. T2.

1877. Larva. C. Thomas. Trans. Dept.Agr. Ill., vol. 15, p.9)!.

1877. Lav'a. Freneh. Trans. 1)ept, Agr, 11l., vol, 15, 13.211.

1879. Larva. G.J. Fonles, („unotes ialey.) Rept. Fintom. Soe. Ontario, p. 41.

1880. Larva. J. Marten. 'Trans. Dept. Agr. HH. vol. 18, A ppend., p. 131.

1880. Larva, (brief.) Riley. Aner. Entomologist, 2d series, vol. 1, 1. 298.

188:. Larva. IF. F. Kiblby. Europ. lintt. and Mloths, p. 191.

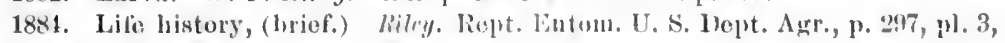
lighe. 1, 2. 


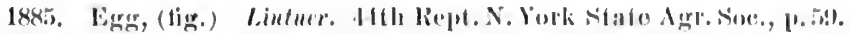

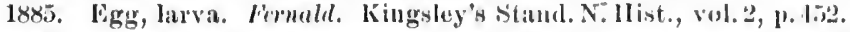

1888. Egg, larva, (itgs.) Cintuer. Bull. N. X. State Musemu, No. (1, Pp. 8, 16.

Agrotis lubricans. Guen.

1880. Larva. French. Canarl. Entom., vol. 12, 1. 14.

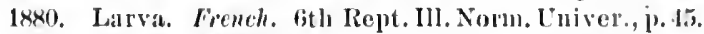

1880. Lav'va. J. Murten. 'Traus. Dept. $\Lambda$ gr. Ill., vol. $18, \Lambda$ ppend., p. 135.

Agrotis prasina. Fabr.

1859. Larva. M. T. Stainton. Brit. Butt. and Motlıs, vol.1, 1. 271.

Agrotis occulta. IIihn.

1829. Larva. Stipliens. Jll. Brit. Entonı., Haust., vol. 3, p. 29.

1829. Iarva, (col'd figs.) Curtis. Brit. Enton., vol.6, pl.218.

18.9. Larra. II. T. Stainton. Brit. Butt. and Moths, vol. 1, p. 271.

18.5). Lanra, (col'd fig.) IIumphreys. (ienera Brit. Moths, vol. 1, pl. 21.

1882. Jarva. W. F. Firby. Enrop. Jutt. and Motls, 1). 207.

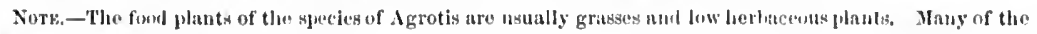
Aluecis's are omuivorots.

Adita chionanthi. $\Lambda \mathrm{bl}$. - Sin.

1797. Larva, (col'd tï.) Abhot-Smith. Lep. Ins. (icorgia, p. 195, pl. 98.

Fond Plant. Chiomanthus rirginice.

Mamestra adjuncta. Boisd.

1872. Larva, Lintner. 26th Rept. N. York State Cab. N. Iist., p. 161.

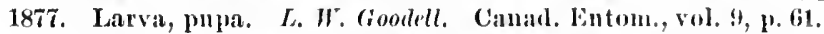

1880. Larva. J. Mart'n, (quotes Goollell.) 'T'ans. Dept. Igr. Ill., vol. 18, Append., p. 136.

Foon Piant. Solidago.

Mamestra assimilis. Morrison.

1881. Linva. L. $\mathbb{H}^{r}$. Goodell. Papilio, vol. 1, P. 15.

Foon Plaxt. Solidago

Mamestra picta. Harris.

1811. Larva. Harris. Ins. Inj. Vegetat, , 1st edit., p. 329.

1852. Larva. Inervis. Ins. Inj. Vegretat., 2 d ealit., p. 3ino.

1862. Larva, mpa, (figs.) Harris. Ins. Inj. Vegetat., Flint's enlit., 「’. 151.

1870. Life history, (fig.) Riley. 2d Missonri Rept., p. 112.

1871. Larva, (fig.) Bethune. Rept. Entom. Soc. Ontario, p. 426.

1872. Larva. Lintuer. 26th Rept. N. York State Cal. N. Ilist., p. 137.

1876. Larva. C. Thomas. Trans. Dept. Arrr. IH., vol. 11, p. 130.

1877. Larva. French. 'Trans. Dept. Agr. Ill., vol. 15, p. 226.

1877. Iarva, (fig.) Paekarl, (af'ter Riley.) Inj. Ins. Wost, Jayden's Rept., p. 753.

1878. Larva, (fig.) .J. Staffer. Agr. Pennsylv., p. 239.

1879. Larva. C. Thomas. 'Trans. Dept. Agr. Ill., vol. 17, Appond., p. 51.

1880. Larva, (fig.) D. Coquilletl. 'Trans. Dept. Agrr. 11., vol. 18, Append., p. 185.

1883. Lifo history, (figs.) Riley. Rept. Entom. IT. S. Dept. Agr., [\%. 124, p]. 1, fig. 3, and pl. 12, fig. 2.

188.I. Lirval stages. Paekard. Amer. Naturalist, vol. 18.

1885. Larva. Fermald. Kingsley's Stand. N. IIist., vol. 2, p.452.

1888. Larva, (lig.) Linlner. Bull. N. Y. State Museum, No. 6, p. 21.

Food liants. Varions.

Mamestra grandis. Boisl.

1883. Larva. Thaxter. Papilio rol.3,p.17.

Food Plant. Arctium lappa.

Mamestra subjuncta. G, aud R.

1869. Larva. Riley. 1st Missouri Ropt., p. 85. 


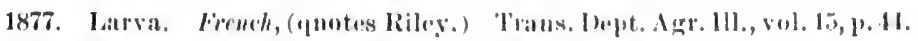

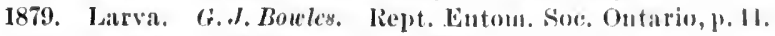

1880. Larva. J. Marten. Trans. Dept. Agr. 111., vol. 18, Append., p. 136.

Foon Plaxts. Cruciferer.

Mamestra distincta. Ilïbn.

1880. Larva. French. Gth kept. Ill, Norm. Luiv., p. Li.

1880. Larva, pupa. J. Martia. 'Traus. Dept. Agr. Ill., vol. 18, Append., p. Jiti.

Food Plant. ritis.

Mamestra laudabilis. Gneii.

1852. Larva, pmpa. Gunn., (guotes Abbot in lif.) Spoce (icuer Noet., vol, 2, р. 30 .

Foon I's.sints. Leyuminosor.

Mamestra trifolii. Rott.

1881. Larra. I. Coquillett. Papilio, vol. 1, 1. 7.

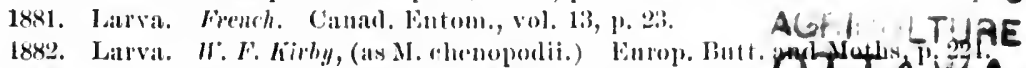

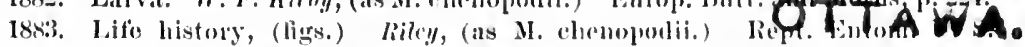
Dept. Agr., p. 121, pl. 1, figg. 5, and pl. 1:, lig. 1, (an M. trilolii.)

1888. Lavia, pupa, (figs.) lintuer. liull. N. York State Museum, No. (i, p.9. Foon Puaxts. Chenopoliacere.

Mamestra renigera. Steph.

1869. Larva, (fig.) likly. 1st Missouri Rept., p. 86.

1877. Latrva, (lid.) French. Trans. Dejt. Agr. Ill., vol. 15, p. 215.

1879. Lava, (hrief.) G. J. Boules. Rept. Eutom. Soc. Outario, P. 45.

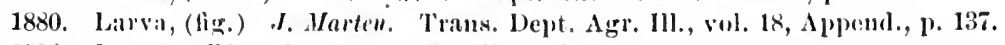

1883. Lavia. Eluards-Elliot. Papilio, vol, 3, p. 133.

FOOd P'l.s.t. Cichorium intylus.

Hadena devastatrix. Brace.

Larva. Brace. Silliman's Jour. Se. and Art, rol. 1, p. 1:s.

1852. Larva. Marris, (cuotes Brace.) Ins. luj. Veretat., Qi erlit., p. 345.

1862. Lacva. Ilarris, (quotes Brace.) Ins. Inj. Vegetat., Flint's edit., p. 4t5.

1869. Larva, (tig.) Riley. 1st Missouri hept., p. 83.

1877. Larva. C. Thomas. Trans. Dept. Agr. Ill., vol. 15, 1. 96.

1877. Litva. French, (yuotes Riley.) Trans. Mept. Agr. Ill., vol. 15, p. 216.

1879. Larva, (fig.) G. J. Boules, (quotes liarris.) Rept. lintom. Soe. Ontario, p. 38.

1880. Larva. J. Marten. Trans. Dept. Agr. Ill, vol. 18, Append., p. 137.

1833. Larva, (fig.) Sannders. Ins. In.j. Fruits, 1. 329?.

1884. Life history, (brief.) Riley. Rept. Eutom. U. S. Dept. Agr'., 1. 2!6, pl. 3, figs. 3,4 .

1885. Larra, (fig.) Lintuer. 44th Rept. N. York State Agr. Soc., p. 5x.

1888. Larva, (fig.) Lintuer. Bull. N. York State Musonu, No. 6, p. 5.

Food l'taxts. Crucifere', (ehietly).

Hadena arctica. Boist.

18i2. Larva. Haris, (as II. amiea.) Ins. Inj. Vegetat., 2ll edit., p. 239.

1856. Larva. Fitch. 3d Rept. Ins. N. York, p. 125.

1862. Larva. Iarris. Ins. Inj. Vegetat., Flint's alit., p. 150.

1865. Larva. B. D. Walsh. Prae. Eutom., 1). 22.

1869. Larva. Harris. Entom. Corres., 1, 316.

1869. Eggr. Minot. Canal. Entom., vol. 2, p. 28.

1870. Larva. Saunders. Canad. Entom., vol. 2, p. 75.

1877. Carva. C. Thomas. Trans. Dept. Agr. 111., vol. 1i, 1., 96.

1877. Larva. French. 'I'rans. Dept. Agr. Ill., vol. 15, p.217.

1879. Larva. G. J. Bonles, (quotes Harris,) Rept. Futom. Soe. Fintario, p. 38.

1880. Larva. J. Marten. 'Trans. Dept. Agr. Ill., vol. 18, Apperul., 1. 137. 
Hadena remissa. Iliilon.

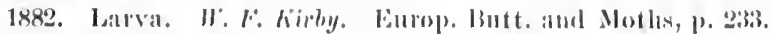

Hadena furbulenta. Ilijhn.

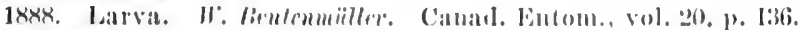

Fown l's.s.r. similex".

Dipterygia scabriuscula. Linn.

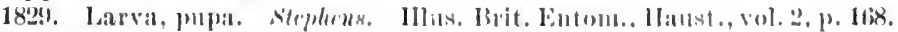

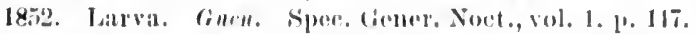

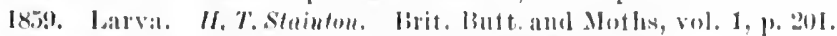

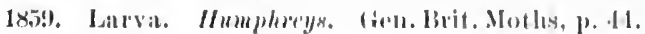

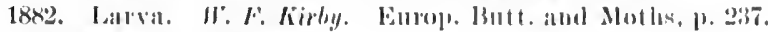

Foub I'Last. linmex.

Hyppa xylinoides. rincru.

1869. Lifi history. Situmtirs. Canad. bintom, vol.2, p. 33.

1888. Lanvi, (hilef.) Liature, th Rept. St t1e lintom., p. 138.

Foon l'tasts. Virions.

Polia vorax. Behrens.

1881. Larva, (brief.) .J. Rethrus. Papilio, vol. 1, p. 21.

Laphygma frugiperda. $\Lambda$ h, ...Sm.

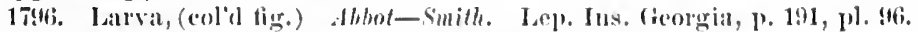

1852. Larva. Gurn, (fluotes Abbot.) Spere (iener. Xoct., vol. 1 p. 159.

18it. Eggr, larva, (ligs.) Rilly. 3il Missomri Repu., p. 11.2.

187i. Larva, (ligg.) lifley. Sth slissomri Ropt.e p. 18.

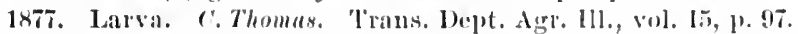

1877. Larva, Frneh. Trans. Dept. Ant. Ill.; vol. 1i, p. 21!.

1881. Larvi, (fig.) .J. Marten. 'l'rans. Dept. Agr. Ill.. vol. 18, Append., p. 138.

1885. Eigg, larva, (fig.) Hubletrd. Ins. Allecting Oranges, p. 150.

1885. Larva, pupas. Cinndluch. Entom. Cubana. p. 288.

1888. Larva, (lig.) Lintuer. Bull. N. York State Mnscum, No. 6, p. 14.

Foob Piants, Cereuls, de.

Prodenia phytolaccæ. Ahb.-.Sn.

1797. Larva, (eolil lim.) Abbot-Smith. Lep. Ins, Georgia, p. 1!3, ph, 97.

Foop Plaxt. I'hylolucen.

Prodenia commelinæ. A hb.-Sm.

1796. Larva, (eol'd fig.) Albot-Smith. Lep. Ins. Georgia, pr. 189, pl. 9\%.

1852. Larva. Guen, (quotes Ab],-Sin,) spee, (iener, Noct., vol. 1, p. 162.

1871. Larva, (fig.) Riley. 3el Mismonri Rept., p. 113.

1877. Larvi. Fremli. Trans. Dept. Agr'. Ill., vol. 15, p. 220.

1880. Larva. J. Marten. 'Trans. Dept. $\Lambda$ gr. Ill., vol. 18, $\Lambda$ ppenl., p. 138.

Foon l'lants. Cereals.

Prodenia lineatella. Harvey.

1880. Larva. J. Marten. Trams. Dept. Agr. I1l., vol. 18, Appeni., p. 139.

1881. Larvia, freneh. Camal. Entom., vol. 13, p. 2 .

Foon Plasts, liulus, Tringopogon, cte.

Euplexia lucipara. Limn.

1829. Larva. Stephens. Illus. Brit. Entom., Hanst., vol. 3, p. 4.

1836. Latra, (col'il lig.) Curtis. Brit. Entum., vol. 13, pl. 619.

1852. Larva. Gin'm. Spee. Gener. Noct, vol. 2, p. lis.

1859. Lalia. H. T. Stainton. Brit. Butt. and Motles, vol. 1, 1. 270.

1859. Larva, (colil fig.) Hemphreys. (iener. Brit. Moths, vol. 1, pl. 20.

1882. Larva. T. F. Kirby. Enrop. Butt. and Moths. 1. 237.

1883. Larva. Edlearls-Elliot. Papilio, vol, 3, P. 133.

Foon Prants. Fiburnum, Betula, ete.

Nephelodes minians. Guen.

1877. Larva. C. Thomas, Trans. Dept. Agr. Ill., vol. 15, p. 99.

1877. Latrva. French. Trans. Dept. Agrr. Ill, vol. 15, 1. 220). 


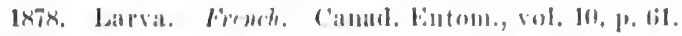

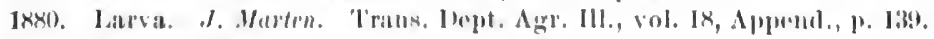

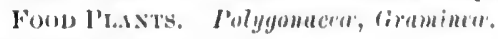

Nephelodes violans. (iuru.

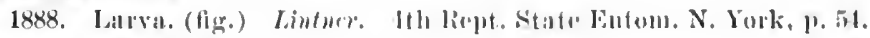

Apamea nictitans, likil.

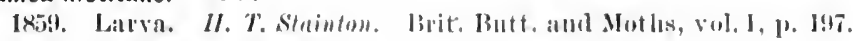

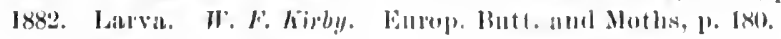

Foon l's.exts. Gromimer.

Apamea immanis. (inch.

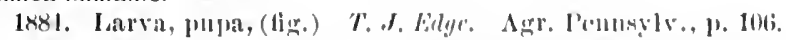

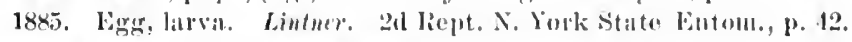

Gortyna Harrisii. (il:= leucostigma.

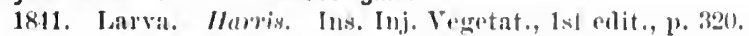

18502. Larva. Marris. Ins. Inj. Vegetat., 21 alif.. l. 311.

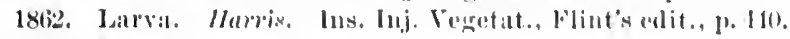

Foob P'I.ast. Agrilegia.

Gortyna nitela. (imen.

1869. Jalval stages, (figs.) likley. 1st Missouri Rept., 1. 92.

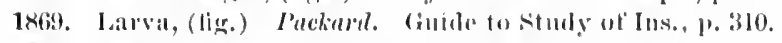

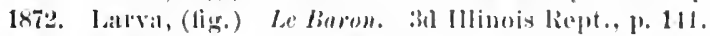

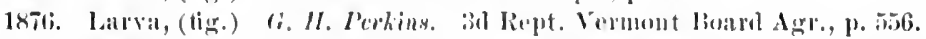

1877. Lavil, pupa. Emily A. Swith. 'Taus, 1)plt. Agr. I11., vol. 15, p. 113.

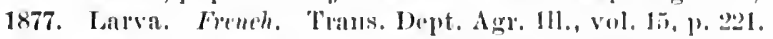

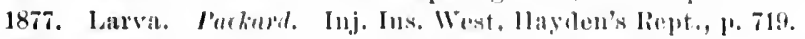

1879. Latra, (tig.) Lim(ne). Ir pt. N. Fork State Anr. Soc., p. 50.

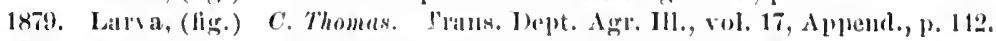

1880. Lanvis, (ligg.) Emily A. Smith. 7th Illinois linpt., p. 112.

1880. Larva, (fig.) I. Coquillett. 'Traus. 1)pent. Agr. Ill., vol. 18, Appomi., p. 1si.

1880. Iarva, (tig.) A. Fullev, (after libley.) Amer. Entom., 2d ser., vol. 1, p.201.

1883. Larva, (fig.) S. A. Forbes. 'Trans. Wiseonsin Agr. Soc., p. 11.

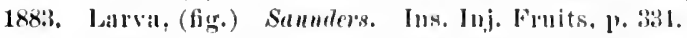

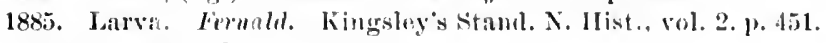

FEeDs in stems of various plants.

Achatodes Zer. Ilarris.

18.11. Lave, Marris. Ins. Inj. Vegetat, Ist edit., p. 319.

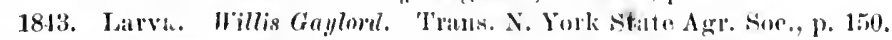

1852. Larva. Haris. Ins. In,j. Vegetat., 2d edit., p. 33:4.

1813. Lalva, pupa. Huris. Ins. Inj. Vegetat., Flint's edit., p. 488.

1867. Lalva. Tenmey. Nat. Hist., 1. 406.

1869. Larva. "rekurel. Guide to Study of Ius., p. 311.

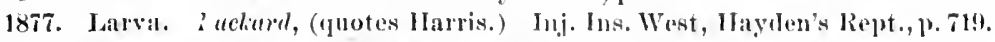

187T. .Larva. C. Thomens. Trans. Dept. Agr. IH., vol. 1\%, p. 100.

1877. Larva. Hench. 'Trans. Dept. Agr. Ill., vol. 15, 1. 220.2.

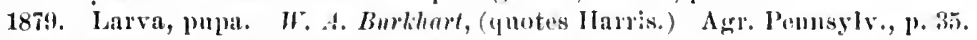

1883. Larva. Edturds-Elliot. Papilio, sol. 3, p. 13.I.

Furos in stems of sambuens, Z'w, etc.

Arzama obliquata. T. and R.

1878. Larva. C. E. Worthington. Canad. Entom, vol. 10, p. 15.

1888. Lifo history, (lirief.) H. H. Brehme. Canal. Entom., vol. 20, p. 119.

Frevs in stems of Typha latifolia.

Arzama melanopyga. Grote.

1881. Larva. .J. H. Comstoel. Pajpilio, vol. 1, p. 147.

Fueds in stalles of Symphara. 


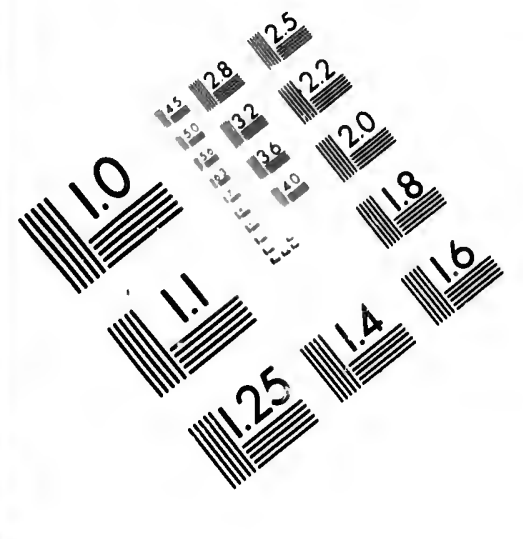

\section{IMAGE EVALUATION TEST TARGET (MT-3)}
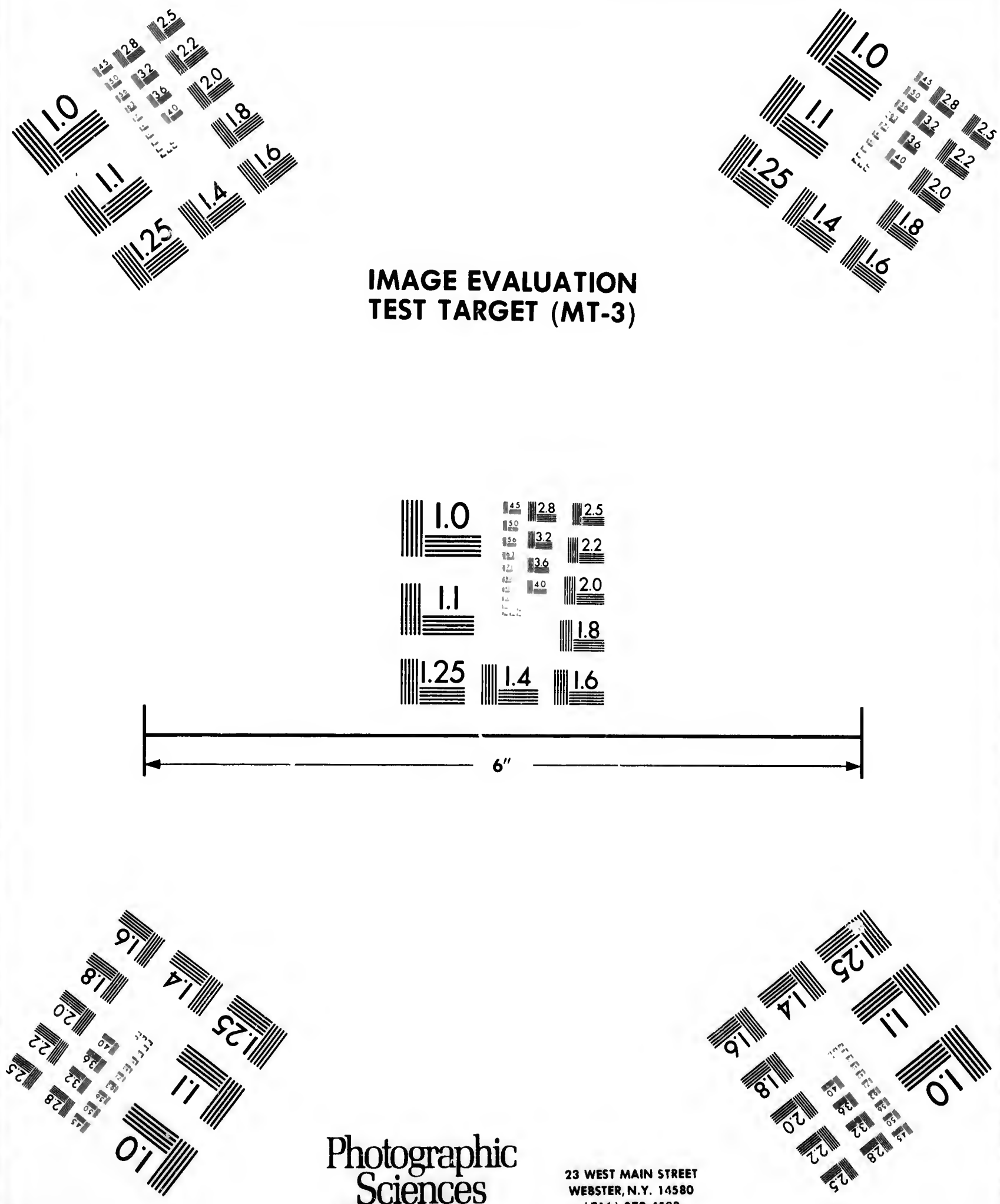

Photographic Sciences Corporation
23 WEST MAIN STREET

WEBSTER, N.Y. 14580 (716) $872-4503$

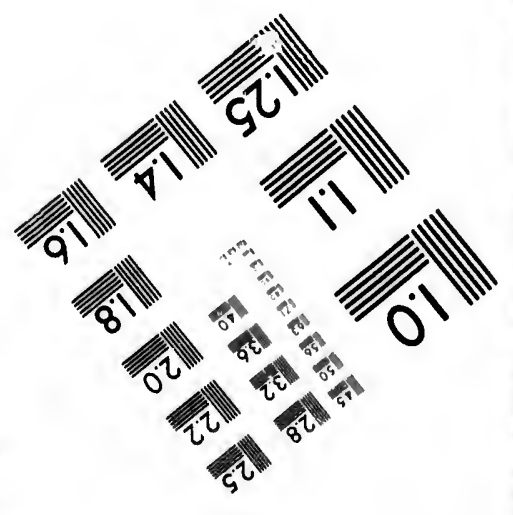



Scolecocampa liburna. Geyer.

1852. Larva, (col'd tig.) Guen, (after Abbot, MS.) Spec. Gener. Ncct., vol, 1, p. 131, pl. 2, (as S. ligni.)

1878. Larva. W. V. Andrews. Psyche, vol. 2, p. 272.

1883. Larva. Edlwardg-Elliot. Papilio, vol. 3, p. 134.

Fewns in decaying stumps of Chestnut and other trees.

Euthisanotia timais. Cramer.

1852. Larva, (col'd fig.) Guen., (after Ablot, MS.) Spec. Gener. Noct., vol. 1, p. 116, pl. 2.

1857. Larva, (fig.) Chenn-Demarets. Encycl .Hist. Nat. Papillons, vol, 2, p. 111.

1886. Larva, pupa. Gundlach. Entom. Cubana, p. 304.

Food Prant. Pancratium.

Monodes nucicolora. Guen.

1852. Larva. Guen. Spec. Gener. Noct., vol. 1, p. 241.

Food Piants. Varions low herbs.

Heliophila pallens. Limu.

1829. Larva. Stephens. Illus. Brit. Entom., Hanst., vol. 3, p. 76.

18ï2. Larva. Guen. Spec. (iener. Noct., vol. 1, p. 93.

1857. Larva. Chenn-Demarets. Encyel. Inist. Nat. Papillons, vol. 2, p. 76.

1859. Larva. II. T. Stuinton. 13rit. Butt. and Moths, vol. 1, p. 190.

1882. Larva. W. F. Kirby. Europ. Bntt. and Moths, p. 160.

Food Plants. Graminea.

Heliophila albilinea. IIübn.

1877. Life history, (figs.) Rilcy. 9th Missouri Ropt., p. 50.

1877. Larva. French, (quotes Riley.) Truns. Dept. Agr. Ill., vol. 15, p. 223

1880. Larva. D. Coquillett. 'Trans. Dept. Agr. Ill., vol. 18, Appond., p. 186.

Food Piants. Cercals.

Heliophila phragmitidicola. Guen.

1880. Larva. French. 6th Rept. Ill. Stato Norm. Univ., p. 46.

1880. Larva. D. Coquillett. 'Trans. Dept. Agr. Ill., vol. 18, Append., p. 186.

Food Plants. Graminee.

Heliophila pseudargyria. Ginen.

1874. Larva. Caulfield. Canad. Entom., vol. 6, p. 132.

1880. Larva. J. Marten. Trans. Dept. Agr. Ill., vol. 18, Append., p. 139.

1881. Larva. French. Canal. Entom., vol. 13, p. 24.

Foon Plants. Gramincer.

Heliophila unipuncta. Hawortls.

1860. Larva. J. Ki rkpatrick. Agr. Rept. Ohio.

1860. Larva, pupa. (fig.) B. D. Walsh. Trans. Ill. Agr. Soc., vol. 4, pp. $350,366$.

1861. Larva, pupa. Packard. 6th Rept. Maine Board Agr., p. 132.

1862. Life history, (brief.) Harris. Ins. Inj. Vegetat., Flint's edit., p. 627.

1865. Larva, pupa, (figs.) B. D. Walsh. 'Truns. Ill. Agr. Soc., vol. 5, p. 482.

- 1869. Larva. Packard. Guide to Study of Ins., p. 305.

1870. Lifo history, (figs.) Riley. 2l Missouri Rept., p. 37.

1870. Larva, (fig.) T. Glover. Rept. U. S. Dept. Agr., p. 83.

1871. Lifo history, (figs.) Bethute. Rept. Entom. Soo. Ontario, p. 402.

1875. Larva, pupa, (figs.) A. J. Cook. 14th Rept. Michigan Agr. Soc., p. 280.

1876. Larva, (briff.) Saunders. Rept. Entom. Soc. Ontario, p. 39.

1876. Egg, larva, pupa, (figs.) Riley. 8th Missouri Ropt., pp. 32, 183.

1876. Larva, (brief.) C. Thomas. Trans. Dept. Agr. Ill., vol. 14, p. 56.

1877. Larva. C. Thomas. Trans. Dept. Agr. Ill., vol. 15, p. 81.

1877. Larva, pupa, (fig.) C. Thomas. Trans. Dept. Agr. Ill., vol. 15, p. 101.

1877. Lurvn, pupa. French. Trans. Dept. Agr. Ill., vol. 15, p. 224.

1877. Larva. Paekurd. Half-honrs with Inseets, pp. 184, 217.

1877. Life history, (figs.) Puckard, (quotes Riloy and othors.) Inj. Ins. West, Hayden's Rept., p. 699. 
1877. Ligg, larva, pupla, (figs.) litily. Rept. Massachusedts loard Agr., p. 24ti. 1879. Egg, larva, pupa, (figs.) I. II. Comstock. Rept. U. S. Dept. Agr., p. 187.

1880. Larva, pupa, (tigs.) Riley. Amer. Entom., 2d ser., vol. 1, p. 170.

1880. Life history, (tigs.) Riley. 3l Rept. Fintom. Comm., p. 89.

1880. Larva, (fig.) J. Fletcher. Rept. Entoul. Soc. Ontario, p. 67.

1880. Larva, pupa, (figs.) C. Thomus. 'Trans. Dept. Agr. Ill., vol. 18, Appenul, pp. $5,43$.

1883. Larva, pupa, (figs.) T. J. Edge. Agr. Pennsylv., p. 73.

1885. Egg, larva, (fig.) Fermald. Kingsley's Stand. N. Hist., vol. 2, p. 451.

1885. Life history, (col'd figs.) Riley. th Jept. Entom. Comm.

1887. Larva, (fig.) Fleteher. Rept. Entom. Dept. Agr. Can., p. 11.

1888. I .'va, (fig.) J. Fleteler. Rept. Entom. Bot. Dept. Agr. Canals, p. 11.

Food Plants. Gramince.

Pyrophila tragopoginis. Linn.

1829. Larva. Stephens. Illus. Brit. Fntom., IIanst., vol.2, p. if .

1852. Larva. Guen. Spee. (iener. Noct., vol. 2, p) $\$ 16$.

1859. Larva. H. T. Stuinton. Brit. Butt. and Motlıs, vol. 1, ]. 311.

1859. Larva, (col'l fig.) Hemphreys. Genera Brit. Noths, 1' 4, pl. 17.

1870. Larva. Sanuders. Canad. Entom., vol. 2, p. 73.

1871. Larva. Saunders, (as A. depressus.) Canad. Entom., vol. 3, p. 193.

1871. Larva. Saunders, (as A. depressus.) Rept. Entom. Soe. Ontirio, p. 360.

1883. Larva, pupa. Saunders. Ins. Inj. Fruits, p. 275.

Food Plaxts. Various.

Pyrophila pyramidoides. (inen.,.(and vars.)

1852. Larva. Guen. Spee. Gener. Noet., vol. 2, p. 414.

1871. Larva, (figs.) Niley. 3d Missonri Rept., ply. 73, 75.

1871. Larva, (figs.) Le Baron, (after Riley.) 2d Ill. Rept., p. 56.

1871. Larra, (fig.) Saunders. Rept. Entom. Soc. Ontario, p. 360.

187. Larva. Saunders. Canal. Entom., vol. 6, , 1. $2 \overline{7}$.

1875. Larva. Saunders. Canad. Entom., vol. 7, p. 1.f.

1877. Larva, cocoon, pupa, (fig.) French. Trans. Dept. Agr. Ill., vol. 15, p. 225.

1878. Larva. G. H. Perkins. 5th Rept. Vermont Boarl Agr., p. 273.

1880. Larva, (fig.) D. Coquillett. 'Traus. Dept. Agr. Ill., vol. 18, Append., p. 180.

1883. Larra, pupa, (fig.) Sannelers. Ins. Inj. Fruts, p. 27.t.

1885. Larva. Feruald. Kingsley's Stand. N. Ilist., vol. 2, p. 450.

Food Plants. Titis, Ampelopsis.

Tæniocampa incerta. Fiülın.

1852. Larva. Guen., (as T. hihisei.) Spec. Gener. Noct., vol. 1, p. 355.

1856. Larva. Fitch, (as Ortlı. instabilis.) 3d Rept. Ins. N. York, p. 313.

1859. Larva. H. T. Stainton, (as Orth. instabilis.) Brit. Butt. and Moths, vol. 1, p. 243.

Foon Prants. Querens, Salix, Prunus.

Zotheca tranquilla. Grote.

1878. Larva. Hy. Elwards. Proc. Cal. Acak. Se., Jute.

Food Plant. Sambueus,

Calymnia orina. Guen.

1873. Larva. Sanuders. Canad. Entom., rol. 5, p. 206.

1881. Larva. Packard, (quotes Sammlers.) Ins. Inj. Forest Trees, p. 47.

Food Plant. Querens.

Scoliopteryx libatrix. Limn.

, 1829. Larva. Stephens. Illus. Brit. Entom., Hanst., vol. 3, l. 50.

1852. Larva. Guen. Spec. Gener. Noct., vol. 2, p. 406.

1859. Larva. H. T. Stainton. Brit. I3utt. aud Moths, vol. 1, p. 309.

1859. Larva, (col'd fig.) Humphreys, Geiera Brit. Moths, pl. 25.

1860. Larva, (brief.) Duncan. Natural. Library, vol. 30, p. $23 \%$

1872. Larva, (fig.) J. G. Wood. Insects at Home, p. 481. 
1881. Larva. D. Coquillett. Papilio, rol. 1, p. sti.

1882. Larva, pupa, (briof.) W. F. Kirby. Europ. Butt. and Moths, p. 185.

Foon Plant, Sulix.

Scopelosoma devia. Grote.

188.. Lifo histery. Thaxter. Canad. Enton., vol. 16, p. 33.

Food Plant. Quercus.

Scopelosoma morrisonii. Grote.

188. Life history. Thaxter. Canad. Entom., voi, 16i, p. 30.

Food Plant. Querens.

Scopelosoma vinulenta. Grote.

1881. Life history. Thaxter. Caund. Eutom., vol. 16, p. 32.

Food Plants. Quereus, Azalia.

Scopelosoma walkeri. Grote.

1884. Life history. Thaxter. Canad. Entom., vol, 16, p. 31.

Food Plant. Querens.

Scopelosoma tristigmata. Grote.

188.1. Lifo history. Thurter. Canad. Entonl, vol. 16, p. 33.

Food Plast. Quercus.

Scopelosoma moffatiana. Gr.

1888. Larva. R. F. Pearsall. Enton. Anor., vol. 4, p. 59.

Lithophane antennata. Walker.= cinerea.

1871. Lifo history, (fig's.) Riley. 31 Missouri Rept., p. 135.

1877. Larva. Freuch, (quotes Riley.) Trans. Dept. Agr. Ill., rol. 15, 1. 227.

1883. Larva, (fig's.) Saunders. Ins. Iuj. Frnits, p. 138.

Food Prast. Varions finit trees and frnits.

Lithophane izticinerea. Grote.

1883. Le rva. Eduards-Elliot. Papilio, vol. 3, p. 13氵.

Food Plant. Cerasus.

Calocampa nupera. Lintuer.

1878. Larva. Thaxter. Psyelse, vol. 2, p 122.

Calocampa curvimacula. Morrison.

1878. Larva. Thuxter. Psyehe, vol. 2, p. 122.

Cucullia convexipennis. (i. and $R$.

1869. Larva. Lintuer. 23d Ropt. N. York State Cal. N. Hist., 1. 215.

1872. Larva. Lintuer. 26th Rept. N. York State Cal). N. Hist., p.138.

Food Plaxt. Solilago.

Cucullia asterioides. Gnen.

1872. Larva. Lintuer. 26th Rept. N. York State Cab. N. Hist., p. 139.

Foon Prant. Solilago.

Cucullia intermedia. Speyer.

1869. Larva, pupa, (fig.) Lintuer. 23i Rept. N. York State Cab. N. Hist., p. 213. Foon Plast. Various species of Composita.

Adipsophanes miscellus. Grote.

1880. Larva. D. Coquill'tt. N. Amer. Entom., No. 7, p. 52.

1880. Larva. D. Coquillett. Trans. Dept. Agr. Ill., vol. 18, Apluend., p. 180.

Food Puant. Ferbena.

Crambodes talidiformis. Guen.

1880. Larva. D. Coquillett. N. Amer. Eutom., No. 7, 1. 52.

1880. Larva. D. Coquillett. Trans. Dept. Agr. Ill., vol.18, Append., p. 180.

1883. Larva, cocoon. Edu'urds-Elliol. Papilio, vol. 3, p. 135.

Food Puant. Terbena.

Nolaphana malana. Fiteh.

1855. Larva. Fitch. 2i Rept. Ins. N. York, p. 242.

1856. Larva. Fiteh. 3t Rept. Ins. N. York, p. 343.

1877. Larva. I'ackartl. IIn]f-hours with Inseets, 1.181. 
1883. Larva, cocom. Sanuler. Ins. Inj. Finits, p. 101.

Food Pi,axt. Pyrus, ("ipple.)

Anomis erosa. Hübn.

1852. Larva. Gucn. Spee, (iener. Noct., vol.2, p. 395.

1885. Life history, (col'd tigs.) Ril'y. 4th Rept. lintom. Comn.

1886. Larra, pupa. (iunllach. Entom. Cubani, p. 321.

Food Piaxr. Urena lobatu.

Aletia argillacea. IIiibn.

1852. Larva. Harris. Ins. Inj. Vegetat., 2 d alit., p. 356.

1855. Eggr, larva, pmpu. T. Glorrr. Trums. N. York Stato Agr. Soc, p. 71.

1857. Larva, pupa: liteh. 4th Rept. Ins. N. York.

1862. Larva. Harris. Ins. Inj. Vegetat., Flint's ellit., 1. 457.

1869. Larva. P'ackitul. Guido to Study of Ins., p.313.

1870. Lifo history, (figs.) Riley. 2t Missomri Rept., p.38:

1875. Larvi, pupa. Grotc. Rept. Geol. Alabaua, p.199.

1877. Egg, larva. French. Trans. Dept. Agr. Ill., vol. 15, p. 228.

1877. Egg, larva, (figs.) Packurd, (quotos Riley and Grote.) Inj. Ins. West, ILayden's Rept., p. 775.

1877. Egrg, larva, (fig.) l'ackard. IIalf-hours with Insects, p. 221.

1879. Egr, larvit, pupa, (col'd figs.) J. II. Comstock. Rept. on Cotton Ins., Dept. A gri.. p. 75.

1880. Larva, pupa, (figs.) Rilcy. Amer. Entom., 2a series, vol. 1, p. 6.

1885. Lifo history, (colil figs.) Ril'y. 4th Rept. U. S. Entom. Co:mu.

1885. Egg, larva. Feruuld. Kingsley's Stand. N. Hist., vol, 2, p. 450.

Fool Prants. Malcacel.

Ingura præpilata. Grote.

1883. Larva. Eslwatds-Ealiot. Papilio, vol. 3, p. 135.

Foon Plant. Liquidambar styienciflue.

Ingura delineata. Ginen.

1852. Larva. (iwen., (after Albb., MS.) Spec. (iener. Noet., vol. 2, p. 311.

1852. Larva, pupa. Gil'u. Spee. Gener. Noct., vol, 3, 1) 397.

Food Plant. Taberumontuna laurifolia.

Calpe canadensis. Bethuno.

1878. Larva. Thaxter. Psyche, vol. 2, p. 123.

1880. Larva. D. Coquillett. Canad. Entom., vol. 12, p. 4.

1880. Larva. J. Marten. Trans. Dept. Agr. Ill., vol. 18, Append., p. 137.

1880. Larva. D. Coquillett. 'I'rans. Dept. Agr. Hll, vol, 18, Append., p. 172.

Foon Peant. Thalictrum cormutum.

Telesilla clnereola. Giuen.

1880. Larva. D. Coquillett. N. Amer. Eintom., No. 7, p. 52.

1880. Larva. D. Coquillett. 'Trans. Dept. Agl. Ill., vol, 18, Appentl, 1. 180.

Food Plant. Ambrosia.

Hypsoropha hormos. Hiibn.

1852. Larva. Guen. Spee. Goner. Noct. vol. 2, p. 403.

Food Plant. Dyogpyros virginiaun.

Plusia aereoides. Groto.

1876. Larva, pupa. Thaxter. Psyche, vol. 1, p. 188.

Foob Plant. Spiráa salicifolia.

Plusia balluca. Geyer.

1863. Larva. Satnders. Proc. Entom. Soc. Philad., vol. 2, p. 29.

1872. Cocoon, pupa. Lintuer. 26th Rept. N. York State Cab. N. Hist., p. 163.

1873. Larva. Saunders. Caund. Eutom., vol. 5, p. 10.

1877. Larva. French. Trans. Dept. Agr. Ill., vol. 15, p. 228.

Plusia contexta. Grote.

1883, Lifo his,tory. Thaxter. Papilio, vol. 3, p. 18.

Food Plants. Gramine 
Plusia putnami. Girote. 1883. Larvu, (hrief). Thexter. P'apilio, vol. 3, p. 19.

Plusia dyaus. Grote.

1885. Larva, pupa. Lintner. 2d Rept. Stato Entom. N. York, p. 94.

Plusia precationis. Ginen.

1869. Larva. l'ackurel. Guide to Stndy of Ins., p. 312.

1880. Larva. D. Coquillett. 'Trans. Dept, Agr. 1ll., vol, 18, Append., p. 148.

1881. Life history. D. Coquillett. Canad. Entom., vol. 13, p. 21.

Food Puaxrs. Plantuyo, Arctium, Taraxacum, cte.

Plusia brassicæ. Riley.

1870. Larva, j"ułi, (figs.) Riley. 2l Missouri Rept., p. 110.

1871. Larva, ]upa, (figs.) Bethune. Rept. Entom. Soe. Ontario, p. 247.

1877. Larva, coeoon, pupa, (figs.) P'uckarl. inj. Ins. West, Hayden's Rept., p. 752.

1877. Larva. Fronch, (quotes Riley.) Traus. Dept. Agr. Ill., vol. 15, p.230.

1879. Larvi. C. Thomas. Trans. Dept. Agr. Ill., vol. 17, p. 41.

1880. Larva, cocoon, pupin, (figs.) J. Mavten. 'Traus. Dept. Agr. Ill., vol. 18, Append., p. 1.10.

1883. Life history, (figs.) litey. liept. Entom. U. S. Dept. Agr., p. 119, pl. 11, figs. 2,5 .

1885. Larva, pupa, (fig.) Lintuer. 2d Rept. State Entom. N. York, p. 90.

Food Plants. Irrassica and other Crueifiered.

Plusia hochenwarthii. IIoch.

1882. Larva. W. F. Kïby. Europ. Butt. and Moths, p. 266.

Plusia devergens. Ilübn.

1882. Larva. W. F. Kirby. Europ. Butt. and Moths, p. 266.

Ararta cordigera. 'Tlıub.

1859. Larva. H. T. Stuinton. Brit. Butt. and Motus, vol. 1, p. 293.

Foon Plant. Vaccinium.

Chloridea rhexiæ. Alıb.-Sm.

1797. Larva, (col'd fig.) Abbot-Smith. Lep. Ins, Georgia, p. 199, p1. 100.

1852. Larva. Guen. Spec. Gener. Noct., vol.2, p. 175.

1858. Larva. Duncan. Natural. Library, vol. 22, p. 199.

1880. Larva. French. 6th Rept. 1ll. State Norm. Univ., p. 46.

1880. Larva. liiley. Amer. Entom., 2d series, vol. 1, p. 7.

1886. Larva, pupa. Gundlach. Entom. Cubana, p. 310.

Foon I'lants. Rhexia Virginiea, Sesamum, Nicotiana.

Alaria gauræ. Abl.-Sm.

1797. Larva, (col'd fig.) Abbot-Smith. Lep. Ins. Georgia, p. 197, pl. 99.

1852. Larva. Guen. Spec. Gener. Noet., vol. 2, p. ..:0.

1858. Larva, (col'd fig.) D:uvcan, (quotes'A. and S.) Natural. Library, vol. 22, p. 200, pl. 24.

Food Plant. Gaura biennis.

Rhodophora florida. Guen.

1867. Larva. F"teh. 12th Rept. N. York State Agr. Soc., p. 900.

1869. Larva. Sannders. Canad. Entom., vol. 2, p, 6.

1871. Egg, young larva. Saunders. Canad. Entom., vol. 3, p. 76.

1885. Figg, lurva. Fernald. Kingsley's Stand. N. Hist., vol.2, p. 450.

Food Plant. Flowers of anothera biennis.

Hellothls phlogophaqus. G. and $R$, (and vars.)

1877. Larva. Finch. 'Trans. Dept. Agr. Ill., vol. 15, p. 230.

1881. Latra. 1), Cogaillett. Papilio, vol.1, p. 56.

Food leants. Graminece. 
Heliothis armigera. Hiibu.

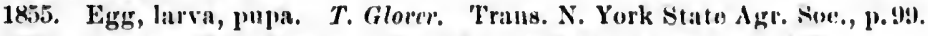

1859. Larva. H. T. Stainton. Brit. Butt. and Sloths, vol. 1, 1, 29!2

18i1. Egg, larra, (fig.) Riley. 3d Missonri Rept., p. 105.

1877. Egg, larva. Packurd. Half-hours with Ins., p. 221.

18i7. Larva. P'ackarl. Inj. Ins. West, Inyden's Ropt., 1. 778.

1877. Larva. C. Thomas. 'Trans. Dept. Agr. Ill., vol. 1;, p. 103.

1877. Latrva, puna. French. Trans. Dept. Agr. Ill., vol. 15, p. 232.

1879. Lifo history, (col't figs.) J. H. Comstock. Rept, Cotton Ins. Dept. Apr., ['. 297.

1880. Egg, larva, coeoon, pupa, (figs.) Coquillett. Trams. Dept. Agr. Ill., vol. $18, \Lambda_{\text {ppenul., p. } 150 .}$

1882. Larva. I". F. Kirby. Lurop. Butt. and Motlss, p. 22\%.

1883. Larva, eocoon, pupa, (figs.) S. S. Rathron. Agr. Penusylr., p. 238.

1885. Lifo listory, (col'l figs.) Riley. 4th Rept. (U. S.) Entom. Comm.

1886. Larva, pupa. Gunilach. Entom. Cubana, p. 309.

Foon Plaxts. Cotton, Resedla.

Pyrrhia exprimens. II ïbn.

1872. Larva. Lintmer. 26th Rept. N. York Stato Cah. N. Hist., p. 163.

1877. Larva. French. Trans. Dept. Agr. Ill., vol. 15, p. 233.

1883. Larva, pupa. Eilwards-Ellict. Papilio, vol. 3, 1. 135.

Foon Plast, Desmodium.

Pyrrhia angulata. Grote.

1881. Larva. Coquillett. Papilio, vol. 1, p. 8.

Food Plaxt. Polygonum.

Tarache erastrioides. Guen.

1881. Larva. Coquillett. Papilio, vol..1, p. 8.

1883. Larva, (brief.) Coquillttl. Papilio, vol.3, p. 84.

Food Plast. Ambromia.

Tarache candefacta. II ïbn.

1852. Iarva, (col'd fig.) Guen. W. Spec. Gener. Noet., vol. 2, p. 26, pl. 2.

1883. Larva, (brief.) Coquillett. Papilio, vol. 3, p. 84.

Food Plast. Ambrosia.

Tarache delecta. Walk.

1870. Larva, pupa, (fig.) Packarl, (as Aeon. metallica, after Abbut.) Amor.

Naturalist, vol. 4, p. 229.

1888. Larva. A. C. Weeks. Entom. Amer, vol. 4, p. 46.

Foon Plant. Hibiscus moschento8.

Chamyris cerintha. Tr.

1881. Larva. Coquillett. Papilio, vol, 1, p. 56.

Food Plant. Pyrus.

Eustrotia carneola. Given.

1881. Larva. Coquillett. Papilio, vol. 1, p. 7.

Foon Plant. Rumex.

Xanthoptera semicrocea. Guen.

1852. Larva, (col'l fig.) Guen., (after Abb., M. S.) Spec. Gener. Noct., pl, 2.

1869. Larva. Puckurl. Guide to Stndy of Ins., p. 316.

1874. Lifo history, (figs.) Riley. Cunad. Entom., vol. 6, p. 208.

Food Plant. Sarracenia.

Exyra rolandiana. Groto.

1877. Larva. Thater. Psyehe, vol. 2, p. 39.

Food Plant. Sarraccnia.

Drasteria erechtea. Cram.

1868. Larva. Saunders. Canad. Entom., vol. 1, p. 4.

186!. Larva, (fig.) Harris. Entom. Corres., pp. 175, 318.

1869. Larva. Packard. Guido to Studly of Ins., p. 317. 
1875. Larva, Saunders. Rept. Entom. Soc. Ontario, 1. 37.

1875. Eggr. Hy. Eillearls. Proe. Cal. Acad. Se, April.

1875. Larva. Scumlers. Canad. Entom., vol. 7, p. 116.

1877. Larva. Frenck. Trans. Dept. Agr. Ill., vol. 15, p. 233.

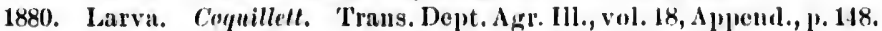

1882. Larva. Scunder's. 12th Rept. Eutom. Soc. Ontario, p. 17.

188. Life history. Freneh. I'apilio, vol.d, p.118.

1885. Larva. Frmald. Kingsley's Staml. N. Ilist., vol. 2, 1. 119.

Foon Piant. Trifolium.

Syneda graphica. Hïlus.

1852. Larva, (col'd fig.) (iuen., (after Abb., MS.) Spec. Gener. Noet., vol. 3, p. 72, pl.2.

Catocala eplone. Drury.

1852. Larvn. Guen. Spec. Giener. Noct., vol. 3, p. 93.

Foon l'Laxt. Cincreus.

Catocala despérata. Guen.= vidua. Ahb.-Su.

1797. Larva, (col'd fig.) Ablot-S'mith. Lep. Ins (Georgia, p. 181, pl. 91.

1852. Larva. Guen., (quotes Abb. _Sm.) Spec. Gener. Nact., vol. 3, p. 95.

1888. Life history. French. Canad. Entom., vol.20, p. 28.

Foon Plaxts. Carya, Qucreus.

Catocala flebilis. Groto.

1881. Larva, pupa. D. hellicott. Pappilio, vol. 1, l. 141.

Food l'bant. Carya.

Catocala relicta. Walk.

1888. Life history. Hoicard L. Clurk. Canad. Entom., vol. 20, p. 17.

Foon Puavr. Salix.

Catocala amatrix. ILübn.

1852. Larva. Guen. Spec. Gener. Noct.,vol. 3, p. 86.

1881. Larva, (brief.) Ph. Fischer. Bull. Buflilo Soc. Nat. IIist., yol. 4, p. 62.

1881. Larva, pupa. 1). Kellicolt. Papilio, vol. 1, p. 142.

1881. Lifo history. French. Papilio, vol. 4, p. 8.

Foon Piant. Populus.

Catocala cara. Ginen.

1881. Larva. A. Koebele, (as C. curissima.) Bull. Brooklyn Entom. Soc., vol. 4, p. 22.

Food Prant. Salix.

Catocala concumbens. Walker.

1863. Larva. Saculers. Proc. Entom. Soc. Philad., vol. 2, p. 29.

Foon Plant, Salix.

Catocala meskei. Grote.

1883. Larva. R. Busker. Canad. Entom.. vol. 15, p. 100.

Food Plant. Populles.

Catocala unijuga. Walker.

1881. Larvi. D. Kellicolt. Canad. Entom., vol. 13, p. 38.

Food Planit. I'opulus.

Catocala parta. Guen.

1883. Larva. Hy. Edwards. Papilio, vol. 3, p. 2.1.

Foon Plant. Sulix.

Catocala innubens. Guen.

1888. Lifo history. French. Canad. Entom., vol. 20, p. 170.

Catocala coccinata. Groto.

1881. Larva. D. W. Coquillett. Papilio, vol. 1, p. 56.

Food Plant. Quereus.

Catocala ultronla. Hübn.

1852. Larva. Guen. Spec. (iener. Noct., vol.3, p.89.

1869. Larva. Puckarl. Guido to Study of Ins., p. 317. 
1874. Laria. Sunnder's. Canad. Eiztom., vol. 6, p. 117.

1879. Larva, pupa, (brief.) Saunlers. Rept. Lutom. Soc. Ontario, p. 74.

1883. Larvı, pupu, (tigs.) Saunders. Ins.Inj. Fruits, p. 177.

Fool loavrs. P'runte, Cormus, $Q_{k}$ "us.

Catocala ilia. Crann.

1852. Larvu. Gum. Spec. Gener. Noct., vol. 3, p. 92.

18i5. Larva. Cuulfichl. Cuuad. Eutom., vol, 7, p. 208.

1881. Larva. A. Koebele. Bull. Brooklyu Entom. Soc., vol.4, p. 22.

1881. Lifo history. French. Conal. Fintom., vol. 16, 1. 12.

Fuod Plasw?. Querent.

Catocala 20e. Behr.

1870. Larva, (briof.) Betr. 'Trans. Amer. lintom. Soc., p.24.

Fools Plant, Queréus.

Catocala neogama. Abb,-Sm.

17:17. Larva, (col'd fig.) Ablot-Smith. Lep. Ins. (ioorgia, pl. 88.

1852. Larva. Guen., (quotes Abh.-Sm.) Spec. (iener, Noct., vol.3, p.96.

1858. Latria, (col'd tig.) Duncun. Natural. Library, vol. 27, 1.203, pl. 26.

Fool Plast. Queveus.

Catocala palæogama. Guen.

1888. Lifo history. French. Canal. Entom., vol. 20, 1. 108.

Foon Plant. Curya.

Catocala muliercula. Ginen.

1852. Larva, (col'd fig.) Ginen., (after Abb., M. S.) Spec. Gener. Noct., vol.3, p.97, pl.2.

Foon Plants. Myrtacea.

Catocala concors. Abb.-Sm.

1797. Larva, (col'd fig.) Abhot-Smith. Lep. Ins. Georgia, 1. 177, pl. 89.

1852. Larva. Guen. Spec. Gener. Noct., vol. 3, p. 99.

Foon Plasts. Myrtacea, Leguminose'.

Catocala polygama. Ginen.

1869. Larva. E. B. Reet. Canad. Entom., vol. :, p. 30.

1876. Larva. Saumlers. Canal. Entom., vol. 8, p. 74.

Foon Piant. Crate'gus.

Catocala cratægi. Sinunlers.

1876. Larva. Sanuders. Cauad. Entom., vol, 8, p. 72.

Foon Prant. Cratagne.

Catocala amasia. Abb.-Sm.

1797. Larva, (col'd fig.) Abbot-Smith. Lep. Ins, Georgia, p. 179, pl. 90.

1852. Larva. Guen., (quotes Abl.-Sm.) spec. Gener. Nuct., vol. 3, p. 104.

1858. Larva. Duncan. Natural. Library, vol. 22, p. 205.

Food Plant. Melia azedaraeh.

Catocala fratercula. G. and R.

1881. Larva. Coquillett. Papilio, vol, 1, p. 7.

Foon Plant. Quercus.

Catocala grynea. Cram.

1880. Larva. Coquillett. Trans. Dept. Agr. Ill., vol. 18, Append., p. 183.

1881. Larva. A. Koebcle. Bull. Brooklyn Entom. Soc., vol. 4, p. 22.

Food Plant. Pyrus.

Catocala amica. Hübn.

1852. Larva. Guen. Speo. Gener. Noct., vol.3, p. 106.

1880. Larva. Coquillett. Trans. Dept. Agr. Ill., vol. 18, Append., p. 182.

1881. Larva. Coquillett. Papilio, vol. 1, p. 7.

Food Piantr. Querchs.

Parthenos nubilis. Hübn.

1869. Lurva. IIaI., Entom. Corres., p. 219.

Foon Prant. Robinia.

IBULL. $35-7$ 
Allotria elonympha. IJiilin.

1852. Larvu. Guen. Spee, (iener. Noct., vol. 3, p. 37.

Foon Plasts. Juglann, Glycine.

Parallelia bistriaris. Hiibn.

1852. Larvn. Gin'n. Spee. Gener. Noct., vol. 3, p. 268.

1870. Larva. Saunder', Canad. Entom., vol. 2, p. 130.

1881. Larva. P'acliaril. Ins. Inj. Forest Trees, p. 113.

1883. Larva. Biderurils-Elliot. Papilio, vol, 3, p. 136.

Foon Plast. Acer rubrum.

Agnomonia anilis. Hiibu.

1852. Larva, pupa. Guen. Spec. Genor. Noct., vol. 3, 1. 274.

Fool, l'aser. Chironia.

Panopoda roseicosta. Ginen.

18:5. Larvi. Gu'n. Spec. Gener. Noct., vol. 3, p. 325.

Food Plant. Jugluns.

Remigia latipes. Guen.

1852. Jarva. Guen. Spee. (iener. Noct., vol. 3, p. 315.

1886. Larva, pupa. Gunllach. Entom. Cubana, j. 35̃4.

Foon P'iant. Hyprieum.

Poaphila flavistriaria. Hülıu.

1852. Larva, pupa, (col'd figs.) Guen. Spec. Gener. Noct., vol. 3, p. 302, pl. 2.

Food Plant. Scufelleriu.

Poaphila sylvarum. Guen.

1852. Larva. Gnet., (from $\Lambda$ bb., MS.) Spee. Gener. Noet., vol. 3, p. 300.

Foon Plant. Andromeda ferruginea. .

Poaphila quadrifilaris, Hübtı.

1852. Larva. Gu'n. Spec. Gener. Noct., vol. 3, p. 300.

Foon Plant. Gogrypium.

Erebus odora. Linn.

1886. Larva, pupa. Gundlach. Entom. Cubana, p. 367.

1887. Larva, (brief.) H. T. Fernall. Entom. Amer., vol. 3, p. 78.

1888. Egg, young larva. H. T. Fernakl, (quotes Wm. Blake in MS.) Entom. Amer., vol. 4, p. 36.

Food Plants. Cassia fistula, Pithecolobium, Saman.

Pheocyma lunifera. Hüibn.

1886. Larva, pupa. Packard. Bull. No.12, U.S. Dept. Agr., p. 22.

Foon Plant. Pinus.

Ypsia aeruginosa. Guen.

1852. Larva, pupa. Guen. Spec. Gener. Noct., vol. 3, p. 17.

Foud Plant. Cornus.

Homoptera edusa. Drury, (and vars.)

1852. Larva, pupa, (col'l fig.) Guen., (from Albb., MS.) Spec. Genor. Noct., vol. 3, pl. 13, 14, pl. 2 .

1870. Larva, pupa, (fig.) Paekard. Amer. Naturalist, vol.4, p. 229.

1870. Larva. Behr, (as H. salicis.) 'Truns. Amer. Entom. Soc., p. 28.

1870. Larva. Behr, (as H. ros,e.) Loc. cit., p. 28.

1882. Life history. French. Canad. Entom., vol. 14, p. 130.

1888. Larva. Lintuer, (quotes Guen.) 4th Report N. Y. State Entom., p. 58.

Food Plants. Rosa, Salix, etc.

Homoptera minerea. Ginen.

1852. Larva, pupa. Guen. Spec. Gener. Noct., vol. 3, p. 15.

Homoptera calycanthata. Abb.-Sm.

1797. Lurva, pupa, (col'd figs.) Ablot-Smeith. Lep. Ins. Georgia, p. 104, pl. 207.

1852. Larva, pupa. Guen., (quotes Abh.-Sm.) Spec. (iener, Noct., vol.3, p. 15.

Foon l'bant. Calyenutlun fterillus. 
Pseudanthræcia coracias. (inon.

1852. Larva, (col't thg.) Gucu. Speo. Gener. Noct., vol.3, p. 19, pl.9.

Foot l'tant. Querche.

\section{Epizeuxis americalis.}

185. Larva, pupa. Giken. Spee. Gonor. Dolt. Pyral., vol. 8, p. 78.

Foon Pl.ANTs. Leguminoser.

Epizeuxis aemul. Hiilm.

1870. Larra, mpa, (figs). I'ackurel, (as Holia memulalis-after Abbot, MS.)

Amor. Naturalist, vol. 4, p. 229.

1886. Larva, pupa. I'uckierid. Rejt. Butoun. U. S. Dept. Agr., p. 3396.

Foob p'idxt. Phlor.

Pseudaglossa lubricalis. Geyer.

1880. Larva. J. Marten. 'Trans. Dept. Agr. Ill., vol. 18, Ajpentl., p. 138.

1880. Larva. Coquillett. 'Trans. Dept. Agr. III., vol. 18, Appentl, p. 182.

1880. Iarva. Coquillett. Cunal. Entom., vol. 12, p. 41.

Foon Plants. Gramince.

Chytolita morbidalis. Guen.

1880. Larva. Coquillett. Canad. Entom., vol. 12, p. 41.

1880. Larva. J. Murten. 'l'ruus. Dept. Agr. 1ll., vol. 18, Append., p. 138.

1880. Larva. Coquillett. Loc. cit., p. 182.

Foon Prants. Corylus, Graminees.

Philometra serraticornis. Grote.

1877. Larva, pnpa. French. Trans. Dept. Agr. Ill., vol. 15, p. 216.

Faeds on roots of grasses.

Hypæna evanidalis. Robinson.

1856. Larva, pupa. Fitch, (as II. humuli.) 2(l kept. Ins.-N. York, p. 324.

1880. Larva. Coquilletl. 'Trans. Dopt. Agr. Hll., vol. 18, Append., p. 148.

Food Plant. IIumulus.

Hypæna scabra. Fabr. = humuli. Harris.

1841. Iarva. Ifaris. Ins. Inj. Vegotat., 1sit edit., p. 315.

1862. Larva, cocoon. Harrie. Ins. Inj. Vegetat., 2d edit., p. 372.

1862. Larva, cocoon. IIarris. Ins. Inj. Vegetat., Flint's edit., p. 477.

1868. Larva, pupa, (figs.) Paekard. Amer. Naturalist, vol. 2, p. 333.

1869. Larva, (fig.) P'ackard. Guido to Stucly of Ins., p. 327.

1869. Larva. Harris. Entom. Corres., p. 322.

1872. Larva. Bethune. Repit. Entom. Soc. Ontario.

1877. Larva, pupa, (figs.) P'uckarel. Inj. Ins. West, Iayden's Rept., p. 773.

1877. Larva. French, (quotes liothune.) 'Trans. Dept. Agr. Ill., vol. 15, p. 245.

1879. Larva, pupa. J. I. Comstock. Rept. Entom, Dept. Agr., p. 252.

1880. Larva. Coquillett. Canal. Lintom., vol. 12, p. 43.

1880. Larva. Riley. Amer. Entom., 2d series, vol. 1, p. 8.

1880. Larva. Coquillett. Trans. Dept. Agr. Ill., vol, 18, Append., 1. 148.

1881. Life history. Coquillell. Canad. Entom., vol. 13, p. 137.

Foon Prants. Humulus, Trifolium, itc.

Hypæna madefactalis. Guen.

1854. Larva. Guen. Spec. Gener. Dolt. Pyral., vol. 8, p. 35.

Foon Plant. Alisma plantayo.

\section{GEOMETRID/E.}

Choerodes clemitaria. $\mathbf{\Lambda b b}$.-Sm.

1797. Larva, (col'd fig.) Abbot-Suith. Lop. Ins. Georgia, p. 201, pl. 101.

1876. Larva. Packurd. Monogr. Geometr., p. 562.

1887. Larva, (brief.) D. Bruce. Entom. Amer., vol. 3, p. 47.

Food Plasts. Ulmus, Clematis, etc.

Choerodes transversata. Drury.

1833. Larva, (col'l fig.) Titian P'ule. Lep. Amerieana, pl. 9. 
1876. Isarvu, jupa, (ligw.) I'nckard, (after Ml,hot'n MS. drawing.) Monogr. (iemetr., p.istil, pl. 13.

1880. Larva, jupa. L. W'. Goodell. Canal. Entom., vol, 12, p. 236.

1881. Larva. I'ueliurd. Ins. linj. Furent 'Trees, 1. 112.

1887. Larva, (brief.) D. Bince. Fintom. Amer., vol. 3, p. 47.

Foon l'taxts. Myrien, (ierunium, deer, clc.

Choerodes falcata. I'uckurd.

1876. Ligg, (brief.) I'tckurd. Monogr. (ieometr., p. 558.

Tetracis ægrotata. Ginen.

187.. Larva, coeoon, pupa. Hy. Éhturals. l'roc. Cal. Acad. Sc., September.

Foon l'basrs. Geranium, liosa, etc.

Tetracis crocallata. (inenl.

1869. Egg, larva. Minot. Cunul. lintom., vol. 2, p. 28.

1876. Larva. I'ackurd, (qunotes Minot.) Monogr. (ieonc „r., ה18.

1879. Larva, puja. L. W. (iootlll. Cauad. Futou., vol. 12, p 193.

1887. Larva, (brief.) D. Bruce. Eutom. Amer., vol. 3, 1. 47.

Foon Prants. Custanea, Rhus, ite.

Tetracis lorata. (irote.

1869. Egg. Minot. Canad. Entom., vol.2, p. 28.

1876. Pupa, (fig.) Packinril. Monogr. Geometr., pl. 13.

1877. Larva, jupa. J. W. Goodrll. Canad. Entom., vol.t, p. 62.

1884. Larva, pupa. Packurl. Amer. Naturalist, vol. 18, p. 935.

Food Plant. Comp,onia usplenifoliu.

Tetracis trianguliferata. Packarel.

1886. Lifo history. French. Canad. Entoun., vol. 18, p. 105.

Food Plaxt. Ribes aureum.

Mctanema quercivoraria. Ginen.

1857. Larva. Guen., (after Ablot's MS. drawing.) Spec. Genor. Plaal., vol. 1 , p. 172.

1876. Larva. Packard, (q̨notes (inen.) Monogre, Geometr., p.545.

1881. Larva, (brief.) Packarll. Ins. Inj. Forest Trees, p.51.

Food Plant. Quercus.

Metanema inatomaria. (iuen.

1887. Larva, (brief.) D. Bruce. Eutom. Amer., vol.3, p. 47.

Food Plant. Populus.

Drepanodes varus. G. aud $R$.

1875. Larva, (fig.) I'uckard. Amer. Naturalist, vol. 9, p. 180.

1876. Larva, pupa, (fig.) Packarl. Monegr. Geometr., p. 43, pl. 13.

1877. Larva, pupa, (figs.) I'ackurd. Half-hours with Insects, p. 270.

1881. Larva, pnpa, (figs.) P'uckarl. Ins. Inj. Forest Trees, p. 246.

Foon Plant. Junipcrus.

Caberodes confusaria. IIiilın.

1876. Larva, pupa, (figs.) Packurd, (after Abbot's MS.drawing.) Monogr. Geometr, pl. 13.

1857. Larva, (brief.) D. Bruce. Eutom. Amer., vol.3, p. 47.

Food Plant. Trifolium.

Ennomos alniaria. Linn.

1859. Larva. H. T. Stainton. Brit. Butt. and Moths, vol.2, p. 15.

1859. Larva, (brief.) Humphreys. Gener. Brit. Motlis, vol. 2, p. 84.

1869. Larva. Newman. Brit. Moths, p.56.

1876. Larva, pupa, (fig.) Packurl, (quotes (Goodell and Scudder in lit.) Monogr. Geometr., p.530, pl.13.

1881. Larva, pupa. Packard. Ins. Iuj. Forest Trees, p. 92.

1882. Larva. W, F. Kirhy. Europ. Butt. and Motho, p. 299.

1887. Egrg, pupa, (brief.) I. Bruce. Eutom. Anier., vol. 3, p. 47. 
1888. Cocoon, (briet.) H. s. crune. lintom. Amer, vol.t, p. 13.

Foon P'baxrs. Betulu, .lluus, C'cstanea, ete.

Ennomos subsignaria. Iliiln.

1866. Lifo history. Grof-lliche. Rept. llrooklyn Hort. Soc.

1866. Larva. H. D. Walsh. Eraet, Enton., p. ј7̄.

1868. Larva. L'uckurl. Anner. Naturalist, vol. 2, p. 333.

1876. Laru. I'uchurl. Monogr. Geometr., p. 528.

1887. Idarva. French. 'Trans. Dept. Agr. Ill., vol. 75, 1. 221.

1881. Larva. I'ickard. Ins. Inj. Forest Trees, p. 62.

1882. Lif̣o history, (brief.) C. L. Dodyc. Cunal. Entom., vol. 11, p. 30.

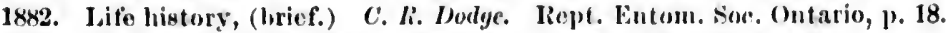

1883. Egg, lavia, pupn, (ligs.) Suunders. Ins. Inj. Fruits, p. 112.

Foon I'lants. Varions trees.

Selenia kentaria. (i. and R.

1887. Kgg, larva, (lirief.) D. Bruce. Entom. Amer., vol. 3, p. 47.

Foou Plants. Betula, decr, ete.

Endropia obtusarla. Hiiln.

1876. Larva, (fig.) I'nckard, (after Abbot's MS. drawing.) Monogr. Geometr., p. 517, pl. 13.

Foon Phaxt. Impatiens.

Endropia pectinaria. W. V.

1876. Isarva. I'ackurd. Momogrr. (ieomotr., p. 513.

1881. Larvu. Packarl, (after Ablot's MS. dlawing.) Ins. Inj. Forest Trees, p. 50 .

Foon Plaxts. Quercus, etc.

Endropia bilinearia. Packard.

1881. Larva, (brief.) I'ackard. Ins. Inj. Forest Trees, p. 19.

1887. Larva, (brief.) I). Bruce. Entom. Amer., vol. 3, p. 17.

Foon Plasts. Prunus, Quireus, ilc.

Endropia armataria. II. Sch.

1869. Larva. Fitch. 13th Rept. Ins, N. York, p. 522.

1871. Larva. Sunnders. Canad. Entom., vol 3, l. 130.

1871. Larva. Sanuders. Rept. Entom. Soe. Ontario, p. 378.

1872. Larva. T. Glorer. Rept. Entom. Trans. N. York State Agr. Soc., p. 83.

188. Larva, pupa. Saunders. Ins. Inj. Funits, 1.351.

Foon Plant. libes.

Endropia textrinaria. G. and $R$.

1869. Larva, pupa, (fig.) Packarl, (after Abbot's Ms. drawing.) Monogr. Geometr., p. 508, pl. 13.

1884. Larva, pupa. I'uckurd. Amer. Naturalist, vol.18, p. 934.

Food Plant. Quereus.

Metrocampa margaritata. Linn.

1859. Larva. H. T. Stainton. Brit. Butt. and Motles, vol. 2, p. 10.

1859. Larva, (brief.) Humphreys. Gener. Brit. Moths, p. 86.

1869. Larva. Ncwman. lirit. Moths, p. 53.

1882. Larva. W. F. Kirby. Europ. Butt. and Moths, p. 298.

Food Prants. Quercus, Betula, Fayns, etc.

Therina fervidaria. Ilübn.

1876. Larva, pupa, (fig.) Packarl, (after Abbot's MS. drawing.) Mouogr. Geometr., p. 494, pl. 13.

1886. Larva, pupa. Packard. Ropt. Entom. U. S. Dept. Agr., p. 329.

Food Plant. Halcsia diptera.

Therina endropiaria. G. and $\mathbf{R}$.

1879. Larva. I. W. Goodell. Canad. Entom., vol. 11, p. 194.

Food Plant, Quercus. 
Therina seminudaria. Walker'.

1886. Pupa. Paclard. Rept. Entom. U. S. Dopt. Agr., p. 329.

Anagoga pulveraria. Linn.

1859. Larvin. II. T. Stainton. Brit. Butt. and Moths, vol. 2, p. 59.

1869. Larva. Merryfield. Newman's Brit. Moths, p. 90.

1876. Larva, pupa. Pinckarl, (quotes Merryfield aud L. W. Goodell in lit.) Monogr. Geometr., 488.

Food Prant. Salix.

Angerona crocataria. Falır.

1869. Larva. Packard. Guide to Study of Ins., p. 319.

1871. Egg, larva, pupa. G. J. Boules. Cunal. Entom., vol.3, p. 9.

1871. Larva, (fig.) Saunlcrs. Rept. Entom. Soc. Ontario, p. 377.

1876. Larva, (fig.) Packard. Monogr. Geometr., p. 475, pl. 13.

1877. Larva. French, (quotes Packard.) 'Trans. Dept. Agrr. Ill., vol, 15, p. 273.

1883. Larva, pupa. S. A. Forbes. 'Trans. Wisconsin Agr. Soc., vol. 31, 1. 6.

1883. Larva, pupa, (figs.) Suunders. Ins. Inj. Fruits, p. 348.

Foo1) Plaxts. Ribes, Fragaria, etc.

Nematocampa filamentaria. Guen.

1869. Larva, (fig.) Harris. Entom. Corres., p. 322, pl. 3.

1876. Larva, pupa, (fig.) Packarl. Monogr. Gcometr., p. 471, pl.13.

1877. Larva. Froch, (quotes Packard.) 'Trans. Dept. Agr. IIl., vol. 15, p. 242.

1880. Larva. Coquillett. Trans. Dept. Agr. Ill., vol.18, Append., p. 149.

1883. Larva, cocoon, pupa, (figs.) Suunders. Ins. Inj. Fruits, p. 167.

1883. Larva. S. A. Forbes. 'Trans. Wisconsin $\Lambda$ gr. Soc., vol. 13, p. 5.

Food Plasts. Ribes, Fragaria, etc.

Hyperetis nyssaria. Guen. = amicaria. H. Sch.

1876. Larva. Sannders. Cauad. Eutom., vol. 3, p. 209.

- 1876. Larva, pupa, (figs.) Packarl, (aftor Ablot's MS. drawings.) Monogr. Gcomtr., p. 464, pl. 13.

1881. Larva. Packard, (quotes Sanndors.) Ins. Inj. Forest Trees, p. 129.

Foon Plants. Fagus, Hypericum.

Aplodes rubrolinearia. Pack.

1887. Larva. Hulst. Entom. Amer., vol. 3, p. 72.

Food Plìnt. Myrica serifera.

Aplodes mimosaria. Ginen.

1864. Larva, pupa, (brief.) B. D. Walsh. Proc. Boston Soc. N. Hist., vol. 9, p. 301 .

1876. Larva, pupa. Packard, (quoces Walsl!.) Monogr. Geometr., 1. 389.

1881. Larva. Puckard. Ins. Inj. Forest Trees, p. 49.

Food Plant. Queicus.

Aplodes packardaria. Grote.

1876. Larva, pupa. Puckard, (as A. rubrifrontalis.) Monog. Geometr., p. 387.

Food Plant. Comptonia asplenifolia.

Aplodes brunnearia. Pack.

1876. Larva, pupa, (fig.) Packerd, (after Abbot's MS. drawing.) Monogr. Geometr., p. 388, pl. 13.

Food Plant. Juglans migina.

Apiojes coniferaria. Pack.

1884. Larva, pupa. Prickard. Amer. Naturalist, vol. 18, p. 933.

Foon Plant. Abies.

Synchlora rubivora. Riley.

1869. Larva, (fig.) Riley. Ist Missouri Rept., p. 139.

1873. Larva, (fig.) Suunders. Rept. Eutom. Soc. Ontario, ?. 16.

1876. Larva. Packard, (quotes Riley.) Monogr. ('eonetr,, p. 382.

1877. Larva. French. Trans. Dept. Agr. Ill., vol. 15, p. 238. 
1883. Larva, pn pa, (figs.) Sesunlers. Ins. Inj., Fruits, p. 316.

Food Piant. Rubues.

Synchlora excurvaria. l'ackard.

1876. Larva, (fig.) Packurll, (after Abhot's MS. drawing.) Monogr. Geometr., pl. 13.

Chlorosea bistriaria. Packard.

1888. Larva. Hulst. Entom. Amer., vol. 3, p. 193.

Food Plant. Solidago.

Eucrostis chloroleucaria. Ginen.

1879. Larva, pupa. Hulst. Bull. Brooklyn Entom. Soc., vol. 2, p. 78.

1880. Larva, pupa. L. W. Goodlll. Canad. Entom., vol. 12, p. 235.

Food Plants. Leucanthemum vulgare, Heliantiuas, ete.

Dysteris abortivaria. II. Seh.

1877. Larva. French. Trans. Dept. Agr. Ill., vol. 15, p. 238.

Food Plant. ritis.

Ephyra pendulinaria. Guen.

1876. Larva, pupa, (fig.) I'ackard, (quotes Sendier in MS.) Monogr. Geomotr., p. 363, pl. 13.

Food Plaxt. Comptonia asplenioidles.

Ephyra myrtaria. Guen.

1877. Larva, pupa. L. W. Goodell. Canad. Entom., vol. 9, p. 62.

Food Plants. Comptonia, Gaylussacia.

Arrhostia lumenaria. IIülon.

1876. Larva, pupa, (fig.) l'ackaril, (after Abbot's.MS. drawing.) Monogr. Geometr., p. 365, pl. 13.

Food Plant, Proralea.

Acidalia insularia. Guen.

1876. Pupa, (fig) Packard. Monogr. ('ieometr., p. 336, pl. 13.

1887. Larva, pupa. Hulst. Entom. Amer., vol. 3, p. 175.

Food Plavt. Cassia chamacrista.

Acidalia enucleata. Ginen.

1857. Larva, pupa, (fig.) Guen. Spee. Gener. Plıal., pl. 12.

1876. Larva, jumpa, (figs.) Packurd, (quotes Ginen.) Monogr. Geomotr., p. 348, pl. 13.

1879. Larva, cocoon. L. W. Goodell. Canad. Entom., vol.11, p. 194.

1880. Egg, young larva. L. W. Goolell. Canad. Entom., vol. 12, p. 235.

Food Plant. Rhexia lutea.

Acidalia ordinata. Walk.

1876. Larva, pupa, (figs.) Packerd, (after Abhot's MS. drawing.) Monogr. Geometr., p. 565, pl. 13.

Food Plant. Trillium stylogum.

Stegania pustularia. Guen.

1871. Larva. Suunders. Canad. Entom., vol, 3, p. 225.

1876. Larva, pupa, (figs.) Packard, (after Abbot's MS. drawing.) Monogr. Geometr., p. 310, pl. 13, (quotes Samulers.)

1881. Larva. Packad, (quotes Saunders.) Ins. Inj. Forest Trees, p. 112.

Food Plant. Aecr.

Eumacaria brunnearia. Packard.

1878: Larva. L. W. Goodell. Canad. Entom., vol. 10, p. 66.

1885. Larva, pupa. D. Kellicott. Canal. Entom., vol.17, p. 32.

Food Plant. Prumes.

Semiothisa præatomata. Haworth.

1857. Larva. Gu'n. Spec. Genor. Phal., vol. 2, p. 76.

1876. Larva. Packard, (quotes Guen.) Monogi. Geometr., p. 293.

Foon) Plast. Vaccinium. 
Semiothsia enotata. Giuen.

1876. Larva, pupa, (figs.) P'ackard, (from Abbot's MS. drawing.) Munogr. Geometr., 1. กtit, pl. 13.

Food Plant. Lactuca grandifolia.

Thamnonoma travaria. Linn.

1829. Larva. Stephens. Ill. Brit. Entom., Hanst., vol. 3, p. 194.

1857. Larva. Gu'n. Spee. Gener. Phal., vol. 2, p.93.

1859. Larva. H. T. Stainton. Brit. Butt. and Moths, vol. 2, p. 56.

1859. Larva, (col'd fig.) Humphreys. Gen. Brit. Moths, pl.31.

1870. Larva. Puckiurl, (quotes (inen.) Inj. Ins. (new or littlo known), p.12.

1882. Larva. W. F. Kirby. Europ. Bntt. and Moths, p. 318.

Foon Plant, libes.

Thamnonoma arunneata. Thunb.

1859. Larva. H.T. Stainton. Brit. Bntt. and Moths, vol. 2, p.62.

Foon Piant. Taccilium.

Eufitchia ribearia. Fitch.

1856. Larva. Fitch. 3d Ropt. Ins. N. York, p. 427.

1865. Larva. B. D. Walsh. Practical Entom., p. 22.

1869. Larva. Harris. Entom. Corres., p. 320.

1871. Larva, pupa, (fig.) Satuder's. Rept. Entom. Soc. Ontario, p. 374.

1873. Larva, pupa, (fig.) A. J. Cook. 12th Rept. Michigan Agr. Soe., p. 143.

1874. Egg, larva, pupa, (figs.) Saunder's. Rept. Entom. Soc. Ontario, p. 19.

1874. Larva. Sanuders. Canad. Entom., vol. 6, p. 138.

1875. Larva, pupa, (figs.) Saunders. Rept. Entom. Soe. Ontario, p. 33.

1876. Larva, (fig.) Packard. Monogr'. Geometr., p. 218, pl.13.

1877. Larva, pupa, (figs.) Packard. Ins. Inj. West, Hayilen's Rept., p. 191.

1877. Lava, pupa. Fivench. Trans. Dept. Agl. Ill., rol. 15, p. 237.

1877. Larva, (fig.) Packartl. Half-hours with Inseets, p. 51.

1877. Egg, larva, pupa, (fig.) Riley. 9th Missonri Rept., p. 3.

1878. Larva, puja, (fig.) G. H. P(rkins. 5th Rept. Vermont Board Agr., p. 263.

1882. Larva, pupa, (fig.) Bethue. 12th Rept. Eistom. Soc. Ontario, p. 83.

1883. Larva, pupa, (fig.) T. J. Eidge. Agr. Pennsylv., p. 74.

1883. Eggr, larva, pupa, (fig.) Saunders. Ins. Inj. Fruits, p. 345.

1888. Larva, pupa, (figs.) Bethune. 18th Rept. Entom. Soe. Outario, p. 56.

Foon Plant. Ribes.

Caripeta angustiorata. Walker.

1884. Larva, pupa. P'ackard. Amer. Naturalist, vol. 18, p. 1045.

Foon Plant. Pinus.

Fidonia notataria. Walker.

1882. Life history. L. W. Goodell. Canad. Eutom., vol. 14, p. 199.

Food Plant. P'inus strobus.

Hæmatopis grataria. Falr.

1859. Life history, (figs.) Ritey. 1st Missonri Rept., p. 179.

1876. Larva, pupa. Pachard, (quotes Riley.) Monogr. Geometr., p. 219.

Food Plast. Nitellavia.

Caterva catenaria. Drury.

1852. Cocoon. Harris. Ins. Inj. Vegetat., 2d edit., p. 358.

1862. Larva, coeoon, pupa, (figs.) Harris. Ins. Inj. Vegetat., Flint's edit., p. 46.

1869. Larva, pupa. Harvis. Enton. Corres., p. 321.

1876. Larva, pupa, (fig.) Packard. Monogr. Geometr., p. 218, pl. 13.

1881. Larva. Coquillett. Papilio, vol. 1, p. ó6.

Foon Plants. Varions slumbs and trees.

Tornos rubiginosaria. Morrison.

1876. Larva, pupa, (figs.) Packard, (after Abbot's MS. drawings.) Monogr. Geometr., p. 564, pl. 13.

Food Plant. Corcopsis. 
Aspilates dissimilaria. Hïlbn.

1857. Larva, pupa. Ginen. Spee. (iet. 'r. Phal., vol. 2, p. 182.

1876. Larva, pupa. P'uchird, (quotes (inen.) Monogr. (ieometr., p. 20\%.

1876. Larva, pupa, (figs.) Packard, (after Ablot's MS. drawings.) Monogr. Geometr., pl. 13.

Food Plant. Trifolimim.

Euaspilates spinataria. Pack.

1887. Larva, (brief.) I. Bruce. Futom. Amer., vol. 3, p. 49.

Cleora pulchraria. Minot.

1870. Larva, pupa. Puckard. Inj. Ins. (new or little known), p. 14.

1876. Larva, pupa, (figs.) Packarl. Monogr. Geometr., p. 4.33, pl. 13.

1881. Larva, pupa. Puckurl, (Inotes Sumblers in lit.) Ins. Inj. Forest Trees, p. 205.

1886. Larva, pupa. Packard. Rept. Entom. Dept. Agr., p. 328.

Foon Plant. Pinus.

Stenotrachelys approximaria. Hübn.

1857. Larva. Guen., (after Albott's MS. drawing.) Spee. (iener. Plial., vol 1, p. 290.

1876. Larva. Packarl, (' 'uotes Gnen.) Monogr. Geometr., p. 449.

1881. Larva. Packirrl. Ins. Inj. Forest 'Trees, p. 49.

Foop Plants. Smilax, Quercus, cte.

Epimecis hortaria. Fall.

1797. Larva, (col'd fig.) Abbot-Smith, (as Ph. liriodendraria.) Lep. Ins. Georgia, p. 203, pl. 102

1876. Larva. Prackard, (after Abbot's MS. drawing.) Monogr. Geometr., p. 4.5.

Food Piant. Liriodendron tulipifera.

Ceratonyx satanaria. Guen.

1853. Larva, (col'd fig.) Guen. Spec. Gener. Plal., vol. 1, p. 194, pl. 2.

1876. Larva. Packaril, (quotes Guen.) Monogr. Geometr., p. 565.

Foon Prants. Liquinambar, Quereus.

Cymatophora larvaria. Guen.

1874. Larva. Saunders. Canad. Entom., vol. 6, p. 34.

1876. Larva, pupa, (fig.) Pachiarl. Monogr. Geometr., p. 438, pl. 13.

Foon Plast. Prunus.

Cymatophora pampinaria. Ginen.

1876. Larva, pupa. Packarl, (qnotes L. W. Goodell in lit.) Monogr. Geonietr., p. 435.

1881. Larva. French. Papilio, vol. 1, p. 82.

Food Piant. Pyrus.

Cymatophora humaria. Giuen.

1876. Larva, pupa, (fig.) Packarl, (after Abbot's MS. drawing.) Monogr. Geometr., p. 437, pl. 13.

Cymatophora crepuscularia. Tr.

185\%. Larva, pupa. Guen. Spee. Gener. Phal., vol. 1, p. 260.

1859. Larva. H. T. Stainton. Brit. Butt. and Motlis., vol. 2, p. 28 .

1876. Larva. Packarl, (quotes Guen.) Monogr. Geometr., 1. 425.

1878. Larra. L. W. Goodell. Canad. Entom., vol. 10, 1. 67.

Foon Prants. Salix, Populus, Aluus, ctc.

Paraphia subatomaria. Gnen.

1876. Larva, (brief.) Packard, (quotes Saunders in lit.) Monogr. Geometr., 1.. 418.

1881. Larva, (briéf.) Packard. Ius. Inj. Forest Trees, p. 20.).

Foon Prant. Pinus.

Paraphia unipunctaria. Haworth.

1857. Larva, pupa, (brief.) Guen., (from Abbot's MS. drawing.) Speo. Gener. Phal., vol. 2, p. 62. 
1858. Larvn. Fitch, (as A. tri, 'spunctatat.) 5th Rept. Ius. N. York, p. 825.

1876. Larva, pupa. I'achard, (quotes (iucn.) Monogr. leometr., p.417.

1881. Larvu. I'uckard. Ins. Inj. Forest 'Trees, p. 48.

Food Plant. Quereus.

Paraphia deplanaria. Ginen.

1884. Larva, pupa. Packard. Amor. Naturalist, vol.18, p.935.

1886. Larva, pupa. I'ackard. Rept. Entom. U. S. Dept. Agr., p. 328.

Food Plast. Abics.

Biston ursarius. Walk.

1876. Lifo history. G. J. Bowles. Canad. Entom., vol. 8, p. 7.

1881. Larva. I'ckiard, (quotes Bowles.) Ins. Inj. Forest 'Trees, p. 121.

Foon, Piant. Populus.

Eubyja cognataria. Guen.

1871. Larva. G. J. Bowles, Canad. Entom., vol. 3, p. 11.

1871. Larva, pupa. Saunders. Rept. Entom. Soc. Ontario, p. 379.

1872. Larva. Lintner. 26th Rept. N. York State Cab. N. Hist., p. 166.

1876. Larva, pupa, (briof.) Packarrl. Monogr. Geometr., p. 414.

1878. Larva. L. W. Goodell. Canad. Entom., vol. 10, p. 67.

1883. Larva. Edwatls-Elliot. Papilio, vol. 3, p. 136.

1883. Egg. A. W. P. Cramer. I3ull. Brooklyn Entom. Soe, vol. 6, p. 48.

18圤. Larva, (fig.) Lintuer. 2 d Rept. N. York State Entom., p. 99.

Foon Plants. Acer, Ribes, etc.

Eubyja quernaria. Abb.-Sm.

1797. Larva, (col'd fig.) Abbot-Smith. Lep. Ins. Georgia, p. 205, pl. 103.

1854. Larva, pupa, (figs.) 'Emmons. Nat. Hist. N. York, vol. 5, p. 246, pl. 36.

1857. Larva. (") Guen. Spec. Gener. Fhal., vol. 1, p. 206.

1876. Larva. Packard, (after Abbot's MS. drawing.) Monogr. Geometr., p. 412.

Food Plasts. Quercus, Cratagus, ete.

Hybernia tiliaria. Harris.

1841. Larvi. Harris. Ins. Inj. Vegetat., 1st edit., p. 342.

1852. Eugg, larva. Harris. Ins. Inj. Vegetat., $2 d$ edit., p. 369.

1856. Larvil. Fitch. 31l Rept. Ins. N. York, p. 343.

1862. Egg, larva. Ifaris. Ins. Inj. Vegetat., Flint's edit., p. 472.

1864. Larva. Jaeger. Life N. Amer. Ins., p. 175.

1876. Larva. Paekard, (quotes Harris.) Monogr. Geometr., pr 410.

1880. Larva. Coquillett. 'Trans. Dept. Agr. Ill., vol. 18, Append., p. 149.

1880. Larva, (fig.) J. H. Comstock, (quotes Coquillett.) Rept. Entom. U. S. Dept. $\Lambda \mathrm{gr}$, , p. 255.

1881. Larva, (fig.) Puekird, (after Comstock.) Ins. Inj. Forest Trees, p. 125.

1882. Larva, (fig.) Sannters. Canad. Entom., vol. 14, p. 22'.

1883. Larva, (fig.) Saunders. Rept. Entom. Soe. Ontario, p. 25.

1883. Eug, larva, pupa, (figs.) Sa Senders. Ins. Inj. Fruits, p. 109.

1885. Egr, larva. Fernald. Kingsley's Stand. N. Hist., vol. 2, p. 446.

Food Plants. Tilia, Pyrus, Ulmus, etc.

Phigalia strigataria. Minot.

1877. Larva. Frenel. Trans. Dopt. Agr. Ill., vol. 15, p. 241.

Food Plants. Rosa, Ulmus.

Phigalia cinctaria. French.

1877. Larva, pupa. French. Trans. Dept. Agr. Ill., vol. 15, p. 241.

Food Plant. I'yrus.

Anisopteryx vernata. Harris.

1796. Life history. Peck. Massachusetts Soc. Prom. Agrie.

1841. Larva. . Hurris. Ins. Inj. Vegetat., 1st edit., p. 332.

1843. Larva. Willis Gaylord. Trans. N. York State Agr. Soc., p. 153.

1848. Larva. Harvis. Prairio Farmer, vol. 8, p. 172.

1849. Larva. Ifarris. Boston Cultivator, vol. 9, p. 376. 
18\%0. Larva. Hurris. N. Engl'd Firmer, ser. 2, vol. 2, p. 2.:3.

18:1. Larva. Harris. Massachuselts P'longhunan, vol. 10, Nos. 8, 333.

1852. Larvin. Harris. N. Engl'tl Farmer, ser, f, rol. \&, p. 15is.

1852. Life history. Ilarris. Ins. Jnj. Vegotnt., 2d edit., 1. 359.

185. Larva Harris. N. Eugl't Farmer, sel. 8, rol. 6, p. 363.

18\%̆. Latva. Fïeh. 3d Rept. Ins. N. York, p. 342.

1862. Lifo history, (tigs.) Hurris. Ins. Inj. Vegetat., Flint,y odit., p. 162.

1862. Larva, (fig.) Packard. 7th Rept. Maine Board Agr., 1. 173.

1864. Larva. Jacger. Life N. Amer. Ins., p. 175.

1867. Latra. Tenney. Nat. IIist., 1'. 407.

1869. Larva, (fig.) Packurl. (inide to Stuly of Ins., p. 321, pl. 8.

1870. Egg, larva. T. Glower. Rept. U. S. Dept. Agr., p. 85.

1870. Lifo history, (figs.) Riley. 2tl Missonri Ropt., p. 94.

1872. Lifo history, (figs.) Le Burom, (after Riley.) Bll Illinois Rept.

1873. Egg, larva, (figs.) A. J. Cook. 12th Rept. Miehigan Board Agric., p. 129.

1875. Egg, larva, (tigs.) Riley. Tth Missouri Rept., 1. 80.

1875. Egg, larval stages, puph, (figs.) Sauuters. Rept. Entom. Soe. Ontario, p. 25.

1876. Egg, larva, pupa. Packarl, (quotes Peck.) Monogr. Geonetr., l. 403.

1876. Larva, pupa, (tigs.) Paekurd. Monogr. Gcometr., p. 405, pl. 13.

1876. Larva, pupa. G. H. Perkins. 2d Rept. Vermont Board Agrie., p. 591.

1876. Llfo history. C. Thomas. Trans. Dept. Aigr. Ill., vol. 14, p. 16.

1877. Egg, larva, pupa, (ligs.) C. Thomas. 'Trans. Dept. Agr. Ill., vol. 15, p. 110.

1877. Larva. French. 'Trans. Dépt. Agr. Ill., vol. 15, p. 237.

1877. Larva, pupa, (figs.) Packurl. Inj. Ins. West, IIayden's Rept., p. 791.

1877. Egg, larva. Packiti. Half-honrs with Insects, p. 173.

1881. Egg, larva, (figs.) Packarl, (after Riley.) Ins. Inj. Forest 'Trees, p. 61.

1882. Life history, (figs.) Riley. Ed Rept. U. S. Entom. Conmn., p. 157.

1882. Lifo distory, (brief.) Bethune, (́quotes Saunder's.) 12th Rept. Entom. Soc. Ontario, p. 84.

1883. Life history, brief, (figs.) Saunders. Ins. Inj. Fruits, p. 67.

1885. Egrg, larva, (figs.) J. Fletcher. Rept. Entom. Dept. Agr. Ontario, p. 23.

1885. Larva. Fernald. Kingsley's Stand. N. Hist., vol. 2, p. 416.

Foon Plants. Ulmus, I'yrus, and many other trees.

Anisopteryx autumnata. Packarl. = pometaria. Maum.

18.1. Larva. Harris. Ins. In.j. Vegetat., 1st edit.,p. 333.

1875. Fgg, larva, (figs.) Riley. 7th Missouri Rept.,p. 83.

1875. Agg, larva, pupa,(figs.) Saunders. Rept. Entom. Soe. Ontario, p. 26.

1876. Egg, larva, pupa, (figs.) Puckurtl. Monogr. Geometr., p. 401, pl. 13.

1876. Larva, (brief.) G. H. Perkins. Rept. Vermont Bonrd Agr., p.595.

1877. Larva. French. Trans. Dept. Agr. Ill., vol. 15, p. 239.

1877. Lgg, larva, pupa, (figs.) Packard. Inj. Ins. West, Hayden's Rept., p. 793.

1880. Egg, larra, pupa, (figs.) Coquillett, (quotes French.) Trans. Jopt. Agr. IIl., vol. 18, Append., p. 148.

1880. Life history, (figs.) Riley. 3d Rept. U. S. Entom. Comm.,p.157.

1883. Egg, !arva, pupa, (figs.) Saunders. Ins. Inj. Frnits, p. 65.

1885. Egg, larva, (figs.) J. Fletcher. Rept. Entom. Dept. Agr. Ontario, p. 23.

1885. Egg, larva. " Ferwald. Kingsley's Stand. N. Hist., vol.2, p. 446.

Food Plants. Pyries, Ulmus, and other trees.

Heterophleps triguttata. II. Sch.

1876. Larva. Packart, (quotes Saunders in lit.) Monogr. Geometr., p. 194.

Foon Plant. Acer:

Hydria undulata. Linn:

1859. Larra. H. T. Stainton. Brit. Butt. ant Moths, vol.2, p. 110.

1859. Larva, (brief.) Humphreys. (Uenera Brit. Moths, p.111.

1869. Lifo histoly. Beauchamp. Newman's Brit. Moths, p. 179. 
1870. Life history. Fiteh. 14th Rept. Ins. N. Yotk, 13. 355.

1876. Larvi. I'uckurd, (quotes leanchum]).) Monogr. Geonetr., p. 173.

1882. Larva. $W^{\prime}, F$. Kirly. Europ. Bntt. and Motlis, p. 378.

Foon Plantr. Prunus, Sulix, ctc.

Rheumaptera fluctuata. Linu.

1859. Larva. II. T. Staint A. Hrit. Bntt. and Moths, vol. 2, p. 102.

1869. Larva. Newman. Brit. Motlis, p.164.

1876. Larva. Packarl, (quotes Newman.) Monogr. Geometr., p. $15 \%$.

1882. Larva. W. F'. Kirby. Europ. Butt. and Moths, p. 378.

Foon Prants. Crucifere.

Rheumaptera unangulata. Ilaworth.

1869. Larva. Newman. Brit. Moths, p. 159.

1876. Larva. I'ackird, (quotes Newman.) Monogr. Geometr., p. 160.

Food Puant. Alsine media.

Rheumaptera tristata. Linn.

1859. Larva. H. T. Stainton. Brit. Butt. and Moths, vol. 2, p. 100.

1859. Larva, (col'd fig.) Ilumphreys. Genera Brit. Motlıs, pl. 43.

1869. .Larva. Niwman. Brit. Moths, p. 157.

1876. Larva. Packarl, (quotes Newman's Brit. Moths,) Momogr. Geometr., p.

163.

1882. Larva. W. F. Kirby. Europ. Butt. and Moths, p. 380.

Food Plast. Galium.

Rheumaptera hastata. Linn.

1829. Larva. Stephens. Illus. Brit. Entom., Haust., vol. 3, p. 249.

1859. Larva. H.T. Stainton. Brit. Butt. and Moths, vol. 2, p. 100.

1859. Larva, (col'd fig.) Humphreys. Genera Brit. Moths, pl. 43.

1860. Larva, (brief.) Dunean. Natural. Library, vol. 30, p. 256.

1869. Larva. Newman. Brit. Moths, p. 157.

872. Larva. J. G. Wood. Insects at Homo, p. 458.

1876. Larva. Paekard, (quotes Newman.) Monogr. Goometr., p. 165.

1882. Larva. W. F. Kirby. Europ. Butt. aud Motlss, p. 380.

1885. Larva, (tig.) Fernald. Kingsley's Stand. N. Hist., vol. 2, p. 447.

Foon Plants. Betula, Myrica.

Ochyria ferrugata. Liun.

1859. Larva. H. T. Stainton. Brit. Butt. and Motlis, vol. 2, p. 105.

1869. Larva. Neoman. Brit. Moths, p. 168.

1876. Larva. Packarl, (quotes Newman.) Monogr. Geometr., p. 148.

Foon Plants. Alsine, Glechoma, etc.

Ochyria munitata. Hübn.

1869. Larva. Newman. Brit. Moths, p. 168.

1876. Larva. Packard,(quotes Nowman's Brit. Motlıs.) Monogr. Geometr.,p. 138.

1882. Larva. W. F. Kïrby. Europ. Butt. and Moths, p. 376.

Food Prant. Senecio.

Ochyria designata. Hübn.

1855. Larva, (col'd fig.) Sepp. Dutch Lepidoptera.

1859. Larva. H. T. Stainton. Brit. Butt. and Moths.

1869. Larva. Nèvman, (quotes Sepp.) Brit. Moths; p. 168.

1876. Larva. Packurd, (quotes Sepp) and Stainton.) Monogr. Geometr., p. 146.

1880. Larva, pupa. L. W. Goodell. Canad. Entom., vol. 12, p. 235.

1882. Larva. W. F. Kirby. Europ. Butt. and Motls, p. 376

Food Prants. Brassiea, Alyssum.

Petrophora diversilineata. Hübn.

1870. Larva. Saunders. Canad. Entom., vol. 2, pp. 74, 128.

1876. Egg, larva, pupa. Packarl.. Monogr. Geometr., p. 128.

1877. Larva. French. 'I'rans. Dept. Agr. Ill., vol. 15, p. 237.

1883. Larvu, pupa, (fig.) Saunders. Ins. Inj. Fruits, p. 271.

1887. Larva, (brief.) D. Bruce. Entom. Amer., vol.3, p. 50. 
1887. Lurva, (fig.) J. Fletcher. Rept. Entom. Dept. Agr. Canall., p. 30.

1888. Latvu, (fig.) J. Fleteher. liept. Entom. Bot. Dept. Agr. Cauada, p. 30.

Fool, P's.exts. Vitin, Amprelopsin, etc.

Petrophora testata. Linu.

1859. Latria. II. T. Stuiutom. Brit. Butt. ant Moths, vol. 2, p. 114.

1868. Larvil. Necmun. Brit. Motlıs, p. 191.

1876. Larva. P'achiral, (quotes Newuinu.) Monogr. (Geometr., p. 123.

1882. Larva. W. F. Kirlhy. Europ. Butt. wnd Moths, p. 370.

Foon Piants. Populus, Sulix, Betula.

Petrophora prunata. Linn.

185!). Larva. IJ. T. Stuintou. Brit. Butt. and Motles, vol, 2, p. 114.

1859. Lalva, pupa, (col's figs.) Humphreys. (ienuin Brit. Moths, pl. 10.

1882. Latra. W. F. Kirby. Europ. Butt. ant Mlotlis, p. 370.

Foon lilant. Ribes.

Petrophora truncata. IIübn.

1869. Life history. Newmen. Brit. Motlıs, p. 186.

1876. Lifo history, (fig.) Packarl, (quotes Newnan.) Monogr. Geometr., p. 109, pl. 13.

Foon Praxt. Fraguria.

Hydriomena sordidata. Falur.

1869. Lifo history. Newman. Bric. Moths, p. 153.

1876. Larva, (fig.) P'achurl, (quotes Newuan.) Monogr. (ieounetr., p. 99, pl. 13.

1882. Larva. W. F. Kirby. Europ. Butt. and Moths, p. 381.

Food Plast. Salix.

Thera contractata. Packart.

1885. Larva, pupa. Packard. Bulletin No. 12, Dept. Agr., p. 22.

Foon Plants. Pinus, Abies, Juniperus.

Epirrita dilutata. Bork.

1858. Larva. Fitch. 5th Rept. Ins. N. York, p. 842.

1859. Larva. H. T. Stainton. Brit. Butt. and Moths, vol. 2, 1' 77.

1869. Larva. Newman. Brit. Moths, p. 109.

1876. Larva, (fig.) Packarl, (quotes Newman.) Monogr. Geometr., p. 88, pl. 13.

1881. Iarva. Packarl. Ins. Inj. Forest Trees, 1) 63.

Food Piants. Prunus, Quercus, Ulmus, etc.

Epirrita cambricaria. Curtis.

1869. Larva. Ncwman. Brit. Noths, p. 76.

1876. Larva. Packard, (quotes Newman.) Monogr. Geometr., p. 86.

Food Plist. Pyrus aucuparia.

Piemyria fluviata. Hübn.

1858. Life history. (Anouymous.) Entomologist's Intelligencer.

1868. Lifo history, (figs.) Milliere. Ann, Linn. Soc. Lyons, p. 50, pl. 90.

1869. Lifo history. Newman. British Moths.

1876. Lifo history, (figx.) Pachard, (цuotes Newman.) Monogr. Geometr., p. 78, pl. 13.

1876. Life history. Packart, (quotes Militiore.) Monogr. Geometr., p. 56t.

Food Plants. Anthemis, Chrysanthemun..

Glaucopteryx cæsiata. Bortl.

1859. Larva. H. T. Stainton. Brit. Butt. anā Motlıs, vol. 2, p. 78.

1869. Lifo history. Newman. Brit. Moths, p. 110.

1876. Lifo hiştory, (fig.) Packard, (quotes Nowman.) Monogr. Geometr., p. 69. pl. 13:

1882. Larva. W. F. Rirby. Enrop. Butt. and Moths, p. 383.

Food Plants. Ericacen.

Glaucopteryx magnoliata. I'aekarl. = cumatilis. G. and R.

1875. Iarva, pupa. Hy. Edwarls. I'roc. Cal. Acad. Se., April.

Fuon l'tuxts. Finchia, (rerauium, itc. 
Eupithecia miserulata. Grote.

1876. Larva, pupa, (figs.) Packart. Monogr. Geomotr., p. 54, pl. 13.

1881. Larva, pupa. Packiarl. Ins. Inj. Forest 'Trees, p. 248.

Fomb Pran r. Junipcrus.

Eupithecia interruptofasciata. Packard.

1881. Larva. Coquillett. Papilio, vol, 1, p. 56.

1883. Larva, pupa. Sannters. Ins. Inj. Fruits, p. 352.

Food Piants. Trifolium, ete.

Eupithecia zygadeniata. Pack.

187i;. Larva. Packard, (quotes Belfrage in lit.) Monogr. Geometr., p. 52.

Foon P'lant. Zygadenus nuttallii.

Eupithecia absynthiata. Linn.

1859. Larva. II. T. Stainton. Brit. Butt. and Moths, vol. 2, p. 69.

1869. Larva. Crewe. Nowman's Brit. Motlis, p. 136.

1876. Larva, (fig.) Packart, (quotes Crewe.) Monogr. Geometr., p. 50, pl. 13.

1877. Larva, pupa. L. W. Goolell. Canad. Eutom, vol. 9, p. 62.

1882. Larva H. F. Kirby. Europ. Butt. and Mothe, p. 403.

Foon l'bants. Varions species of Composite.

Exelis pyrolaria. Guen.

1857. Larva. Guen. Spec. Gener., vol. 1, p. 324.

1876. Larva. P'ackarl, (quotes Giuen.) Monogr. Geometr., p.565.

Food Plant. Pyrola.

\section{PYRALIDE.}

Omphalocera cariosa. Ledorer.

1880. Larva, cocoou. French, (as amphaloma.) 6th Rept. Ill. State Norm. Univor., p. 46.

Food Plant. Anoma triloba.

Asopia farinalis. Linn.

18!1. Larva. Harris. Ins. Inj. Vegetat., 1st edit., p. 343.

1859. Larva. II. T. Stainton. Brit. Butt. and Moths, vol. 2, p. 131.

1877. Larva. French. Traus. Dept. Agr. Ill., vol. 15, p. 247.

1880. Larva. Coquillett. 'Trans. Dept. Agr. Ill., vol. 18, $\Lambda$ ppend., p.157:

1882. Larva. W. $F$, Kirby. Europ. Butt. and Moths, p. 410.

1885. Larva. Fernald. Kingsloy's Stand. N. Hist., vol. 2, p. 444.

Feeds on corn, meal, etc.

Asopia costalis. Fabr.

1870. Larva, pupa, (figs.) T. Glover. Rept. U. S. Dept. Agr., p. 84.

1874. Lifo history, (figs) Riley. Gth Missonri Rept., p. 102.

1877. Larva. Frcuch, (quotes Riloy.) Trans. Dept. Agr. Ill., vol. 15, p. 247.

1882. Larva, pupa, (figs.) Saunders. 12th Ropt. Lintom. Soc. Ontario, p. 45.

Feens on dry clover. Trifolium.

Botis inæqualis. Guen.

1883. Larva. Coquillett. Papilio, vol. 3, p. 102.

Food Plant. Circium lanceolatum.

Botis generosa. G. and R.

1883. Larva. Coqnillett. Papilio, vol. 3, p. 101.

Food Plant. Monarda fistulosa.

Botis tertialis. Ginen.

1854. Larva. Gucn., (after Abbot's MS. drawing.) Spec. General. Delt. Pyral., vol. 8, p. 364 .

Food Plant. Faccinium.

Botis erectalis. Grote.

18R8. Latrva. W. Beutinmialler. Canal. Entom., vol. 20, p. 15.

Fool l'lant. Aporynum. 


\section{APPENDIX.}

Species not distingnishable by modern authors:

\section{NOCTUIDE.}

Hoporina hesperidago. Guen.

1856. Larva. Gueu., (after Abbot MS.) Spec. Gener. Noct. 3, p. 393.

Food Plant, Ostrya virginica.

Hypogramma andromedi:. Guel1.

1856. Larva, (col'd fig.) Guen., (after Abbot MS.) Spec. Gener. Noct. 3, p. 36, pl. 2.

Food Plant. Audromeda arborea.

Nænia typica. L.

1869. Larva. Bethune. Canad. Sutom., vol. 1, p. 87.

(Europenn species not kuown in N. Amer.)

\section{GEOMETRID $\boldsymbol{A}$.}

Endropia pultraria. ínen.

1857. Larva. Guen. Spec. Gener. Plialaen., p. 132.

Geometra siccifolia. Fitch.

1856. Larva. Fitch. 3d Rept. Ins. N. York, p. 381.

For the information of students who may be interested in the transformations of our Lepidoptera, I give references to the following admirable papers, all of which are worthy of the nost careful attention :

Andrews, W. V .......The Cynthia Silk-worm, Amer. Naturalist, vol, 2, 1868.

Bruce, David ......... Food Plants of Geometrida, Entom. Amer., vol. 3, 1887.

BeutenmUlekr, WM..Food Plants of varions Bombyces, etc., Entom. Amer., vols. $1,2,3,1886-88$.

Clemens, B............ Notes on Micro-lepidopterous Larvie, Proc. Eutom. Soc. Philad., vol. $1,1861$.

247.

, p. 45.

Chambers, V. T...... Notes on Amer. Species Lithocolletis, Psyche, vol. 2, 1877.

Food Plants of 'Tineida, Bull. Geol. Survey (Hayden), vol.4, 1878.

Habits of Leaf-mining Larve, Amér. Entom., vol. 1, 2d series, 1880.

Month-parts and Legs of leaf-mining Larve, Amer. Entom., vol. 1, 2d series, 1880.

Edwards, W. H........Experiments with Cold on Phyc. pharos, Canad. Entom., vol. $9,1877$.

Experiments with Cold on Chrysalis of Butterflies, Psyche, vol. 3, 1878.

Effects of Cold on Chrys. of Grapta, Psyche, vol. 3.

Effects of Cold on Larva of L. Disippus, Psyche, vol. 3.

Grote, A. R............Larval Variation, Canad. Entom., vol. 9, 1877.

Lintwer, J. A......... Biography of Hemileuca maia, 26th Rept. N. York Cab. N. Hist., i872.

Mann, B. P............Descrp. Larvw of Lepidoptora, Psyclie, vol. 2, 1877. 
Paсkand, A. S.......... Noter on Family \%ygirnidir, Proe. Finex Inst., vol. 4, 1864.

Larva of Aretiu eaja., Changes of Skin, Entom. Mo., May, vol. 8,1872 .

Eml,ryology of Lep. optera, Amer. Naturnlist, vol. 7, 1873.

Mode of Extrieation of Moths from Cocoons, Amor. Naturalist, vol, $12,1878$.

RILeY, C. V ...........Clover-hay Worm (Asopin costulis), 6th Missourl Rept., 1874.

Yucea Boror (Megathymus yucem), 8th Missouri Rept., 1876.

Pupation of Buttertlics, Proc. Amer. Ass. Acad. Se, vol. 28, 1879.

Canker-worms (their history, ete.), 3d Rept. U. S. Entom. Comm., 1880-82.

Cotton-worm and Army-worm, 4 th Rept. U. S. Eutom. Comm., $1881-85$.

SaUnders, IVM

.IIints ou Describing Caterpillars, Canad. Entom., vol. 2, 1870.

ScUnDER, S. H

Preservation of Caterpillars by Inflation, Amer. Naturulist, vol. $8,1874$.

Fragments of coarser Anatomy of Diurn. Lep., Psyche, vol. 3,1881 .

Pine Moth of Nantucket (Rotinia frnstrana), Mass. Soc. Promotion Agr., 1883.

Trouvelot, L ........ Lifo History of the Species of Silkworm Moths, Amer. Naturnlist, vol. 1, 1867.

WalsiI, B. D .......... On Phytophagic Spoeies and Varieties, Proc. Entom. Soc. Philad., vol. $3,1864$. 
1861.

ay, vol.

1873.

nralist,

1874.

. , 1876 .

vol. 28,

Entom.

Jomtn.,

2,1870 .

urmlist,

ho, vol.

s. Soc.

Natur-

11. Soc.

\section{INDEX TO GENERA.}

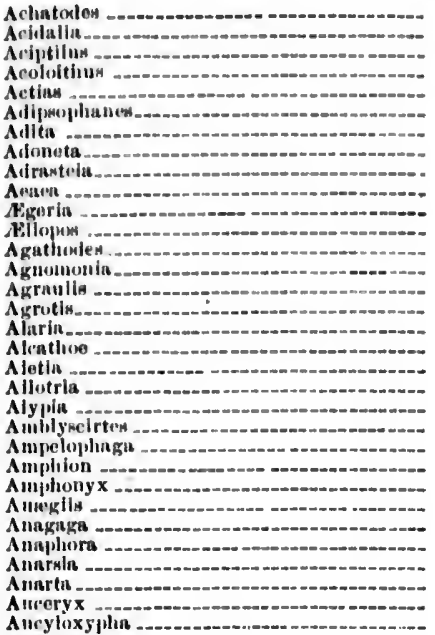

Auroxy

Angeroteryx

Aninota .

Anomik

Anturetin

Anthocerio

Antisjolla

A painea

A patela

A patelones

Aphlomí

Apluieg

A itura

Arachinls -

Aretia.

Argous

Argynnis.

Argyresthin

(1)

Arrihostia.

Arsilonche -

Arzama

Asopina

Atpidieca.

Aepllates

Attucus

Buctra

Batrncliedra

IRedellin.

I\}:mbecia

Blston

Blastobasi

Bombyein

Ilotis

Brentlifa

lineculutrix

Bustatis .

l3yseopliaga

Cuberodes

Cacoecin

Cullidryas.

Callimorpha

Callosamin

Calocempe

Caipe

Calymais
Pago.

\$4

103

1:17

at

71

6if

$12 !$

$1: 30$

51

38

111

08
18

8.1

04

b0)

9.1

98

$4: 3$

38

45

113

120

124

114

34

I) 2

106

76

60

125

89

81

67

113

102

28

61

41

19

126

103

83
89

10

126

105

72

117

129

b)

106

125

80

134

125

155

100

114

15

b6

72

02

91
Page

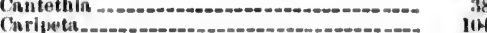

Curifuetal_ar

Cartucesephialus -

C'ustuntegh -

Caturvil al

Cutorain

l'orilontoring -

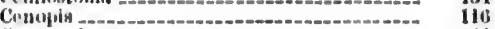

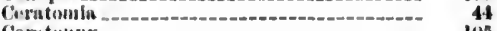

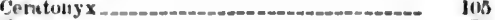

Cerura 70

Chmuу rin__-_.

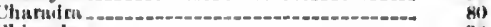

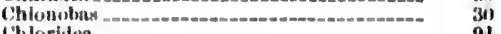

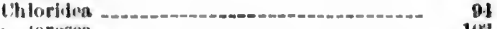

torogen

C uprovalugk - 42

Chourmies -

('horenteg $1: 36$

Chryacory -

Clirysopianus =- 33

Chytollti _... 99

Cinthene

Citlurvulia.

Cirora
Clislociut

C'ydontifituron _...

Coelorlany

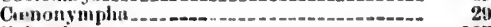

Colenplihorn _- 127

Colcotechnttes

Coling _....- 16

Colorndia _._._._._. 75

Conchylis _.__

Cosmisom - _.

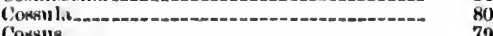

Consus __-

Cramborlest.......-

Crambus -

Cressonin -... 44

Crocotu

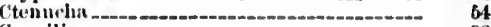

Cucuilia _..._.

Cycloplukis _.

Cymatopliorit -

Ink ruנu, _...

Jhanais -

burehuma -

liasy lof his ...... 6

llatuna

Detile

Dejifanja._.

Depressarla

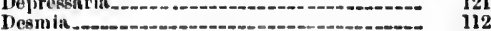

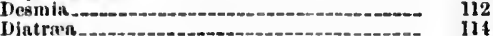

Dichelia._.

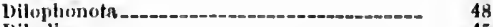

Diludin ...-2 -

Dlpterygia -....

Dolbn

Drasteria

1)repanodes_.

Dryocampa

Dryopteris

Dysteris

Wixcles.

Fecopia

Filemi

(1)

Elachista

Ellemn.

Fimpretin

Findropin

Enzomos
48

70

103

75.

117

61

190

49

65

101 
linge.

Foy 1.

hiplieatia

(1)

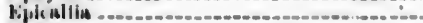

Mpinserim

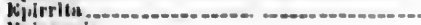

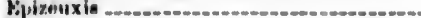

Kribun:

Krevilu - L

Kunajilinte:

Bulıyju.

bibcliatite

Fin.les.

Kijcrontis

Finiln

Kincurvía

Binceron th

Fulloptis

binlryan

Funtebila

Funtacari

Vilumaila

biliplanllionens

Finjitheci

Finjlexia

kinjrepla

hirycreon

(1)

phutix la :

Finstrotia

Rilhinallotio

Fivery $x$

Hxells

Dxy r:

Fatua -

Fonieura

Fulonia

Castropax

Gelechla.

(ieonie' m

Glaucopteryx

Gluphiala

Anuphrela

Cortyun

Gricllaria

Grapholitha

Grapta

IIabroayne

Irailens

llaematopi

Ilalisliota

Ifarmonla

Hiirrlslna ...

IIarrisalmemna

IIrlieonis

uollophlla

ITeilothls

INemaria -

Ilomilence

Il'plalug .

lleterocanipa

Ileteriw.li ros

ITuterojuliegy

(II morn) =Gialeria

IItporine

1fylierula

Ifyil riomons

Ifyilrocemina

11 yloleus

III ypena.

IIyparjux .

1 Iyjerchiria

IIyperetle-

Ilyphantria

Ilyonoment

Hypopreplá

Hy่du
Hyjuompha

Fivinomphis

Incurvarla

Ingurs.

Jnnasen

Junonia lix:

(i)

lik)

Int

ט.

2

Iis

III)

(id)

er

Itis

38

121

117

8:

II

10

3I

111

bi

211

11

bil

91

91

11i

$9 \pi$

bi

31

I1 1

113

122

I:14)

109

6.)

$8:$

126

118

$2: 1$

8

87

114

fil

5

83

17

90

01

37

74

80
70

70

107

98

113

139

100
107

107

112

112
48

48
$9 ?$

68

74

10:

(ii)

134

121

5.5

88

67

67
291

i.

71

bit

J

Lapliygum -

Javerria

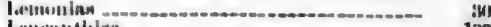

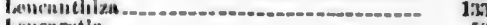

lducuritla

Libythen

Limaruled

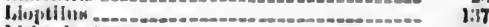

Litharlujitery $x$ _.

Lithoculletio _..._.

Iithophano

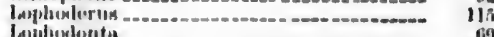

loxulaill

Ly Clina - .

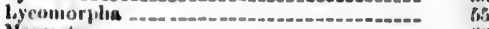

Minulewt ru -

Marmina

Mexathy inima - Men

Melanihrula

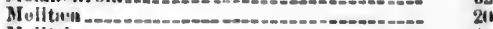

Melittliz

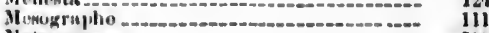

Yietulu

Me( ruxalija

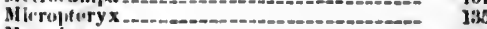

Mlinulew _ _.

Niulata._.__._-_. 68

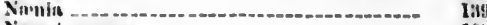

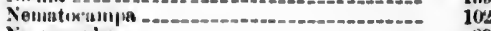

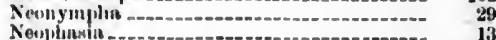

Cepilusliales -

Nivitiuln -

Niaculado

Nuelicliat - -

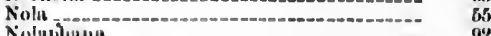

Notliris -

Futislunta

Ochy ria - .

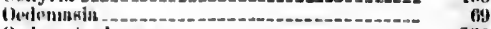

Oedematiphorus

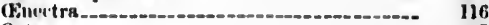

Oeta -

Oiketicus _._.

(iujphiulorera $=-\ldots$

Urgyil _.

Ornix _-n_en 127

Oxyptilus _.

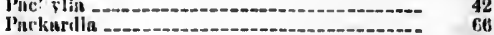

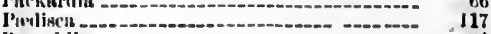

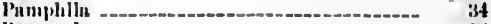

l'anoj)

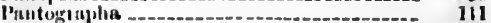

P’ululi

Papilío

I'urallulin . . .

Paruphia

Daraest

Yarnugaing

Parurgyia

Parthenos

Perupelia

Peropliora -

Petrophor:

Phereyma

Plicosia

Milgalia .

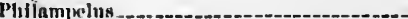

Plillomic

Phlegefliontius -

Plioxopteris

Plivin'tron

Ploslisora.

Plirugunatobia

Phrygunidea

Pliyciules

Pliycis -..--

Phyllocnistis 
INDEX TO GENERA.

Page.

Pierin

13

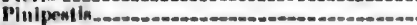

Piacteventicue

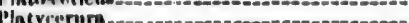

PJutynota

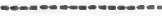

Platypitiling

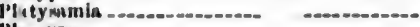

Pleniyra.

1'lusias.

Mlutella

Potplils

(1)

Poxlimenla

Piogovolon - .

Piolla

Prionoxynturn

Promlenia

作

prosloxum.

Ironuba

Previlaklumen

Peoulubarain .

作

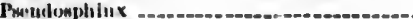

Promelish

Prisyclio

Peychotuoriblia.

Pterophorua

1etycholon

on " -

Pyramein

Pyrophilla _._.

Pyrrhanctia

Pyrrhla

Risphla .

(1)

Jeinigla

Retlnia -

Ihenmaptera

Hhodopliom

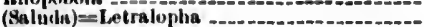

Sannlna

Sarrotlirifa

Satuirula

Satyru:

Scepinis ...

10

(1)

Scolecocamp

Bcolloptery $\mathrm{x}$.

Scoprelonoina

Belrarctla

Belrodonta

Eelenia.

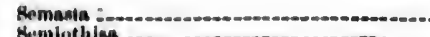

Slay romea -_-

Smoriuthun-.......

Sulenolila

Sphingleanima - 76

Suhlux $\mathrm{x}$. 47

Spiliseonu -

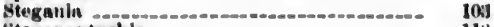

Steknoptychla

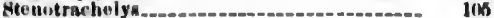

Synchloe 022

Synchilori - 102

Synedis -...-

8ynnoma -

Trenlocamin _._. 01

Tarache -_. 01

Telea 71

Tolesilian....

Terns_-_ons...- 114

Terias n-.....-

Tetracls

Tutralopha -_._.

Theola

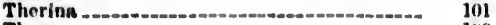

Thers

Thiyreun - - 39

Thyridoptery $\mathrm{x}$

Thyrl6 -

Tinea -

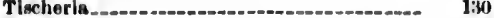

Tmetoceru-_._._. 118

Tolype _............ 79

Tornow -

Tortrix_............... 115

Triptogon -......- 44

Trochillum -....

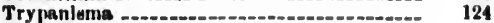

Utetheira_.......... Bit

Vaneesa - -

Xanthoptera -

Xyleatlia

Xylentlia _.

Ypela

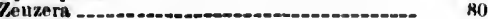

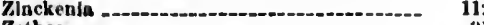

Zotheca 



\section{FOOD-HABIT INDEX.}

Puge.

Ables_.......102, 106, 109, 110, 111, 114, 115, 116, 124

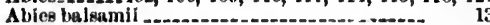
Acacia _82, 61, 63, 65, 72, 77, 82, 100, 10: is, 106,107

Acer psendo-piatunus .................. . . 731,13

Acer ruhrum . .

Acer succharinum-.... 132

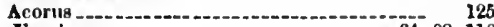

Ascuius Actinomeris

Actinomeris helianthoides

Agrimonia supatoria _.... 123

Ailantilus -

Alisma plantago -

Ainus viridis -

$\begin{array}{lr}\text { Alnus viridis ...... } & 12 \\ \text { Alinine } & \end{array}$

Aisine media

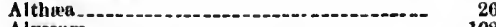

AlybBum

Ambrosia_-36, 03, $95,111,122,123,124,125,130,132$

Ambrosia fagraus 134,137

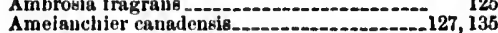

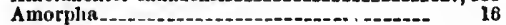

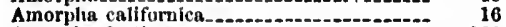

Amorpha fruticosa_._. $39,41,43,53,54,55,91,109,120,133$

Ampelopsis hederacea.................... 39

Amphicarpea monoica-. 133,136

Andromeda arbores

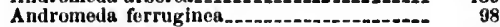

Anoma trifoba.-.

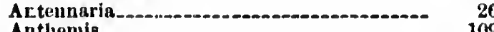

Antliemis -

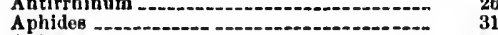

$\Lambda$ pios

Арocyi um -.

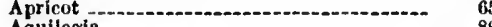

Aquilegia ................................... 89

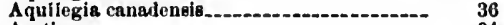

$\begin{array}{ll}\text { Arctium } & \\ \text { Aretium Jappa } & \end{array}$

Arctostaphy los glauca -

Arctostaphylos tomentosa _..._............... 73

Aristolochia -

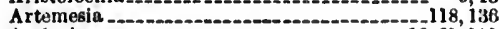

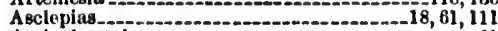

Asclepias tuberosa - 61

Asimina triloba...............................

Asparagub-_.

Astragrius

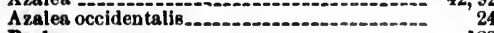

Barley -....-...- 123

Betula -...-..-43, 71, 80,81, 88, 101, 108, 109, 113, 114

Betuia nigra ..... 81

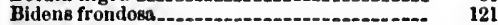

Bignonia - $\quad 48$

Bignonla radicans -

Brachelytrum aristatum 130

Brasica...................... 14, 111,118

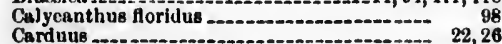

Carduus _... 22, 26

Carya_._ 44, 62, 68, 75, 80, 96, 07, $112,113,115,118,122$

Carya... 11, 02, 68, 75, 80, 06, 07, 112, 126, 128, 132, 135

Carya alba .............................. 83, 125 Cuesia

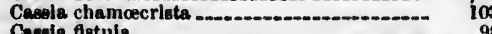
Caseia flatuia - . Cantanea BUน.. 35-10
Page,

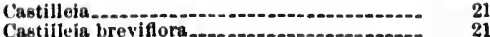

Castifleia breviflors

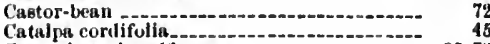

Cesuothus thyrsittorus

Celtís

Ceitis occidentalis -

Ceplajauthus_...

Cephalanthus occidentaiis

Cerasu1......... 11, 12, 27, 64, 70, 81, 92, 115, 116, 12:

Cerasus virginiana...................... 116

Cercis canadensis _..... $\quad \begin{array}{r}122 \\ \text { Cereais }\end{array}$

Cereais

Celtis crassifolia -

Chenojodiacen $\quad 57,87$

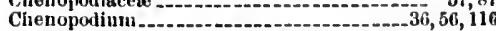

Chest nut and other trees, decayed et umps of, - 90

Chionanthus

Chionanthus virginica ..................... 80

Chironia -..................-. 98

Chrysanthenumum 109

Cícca racemosa -

Cichorium intybue

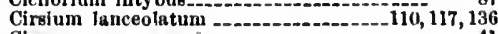

Citrus_ar

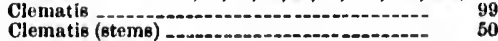

Cleth ra aln!folia-

Clitoria - -

Cnicus -.-.-- 26

Cochlearia armoracia ..... 11

Composite

Comptonia

Coceidwe _._.

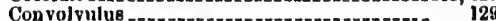

Convoivulus batatas

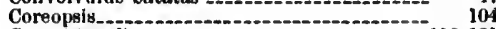

Corn (stored) - ...........

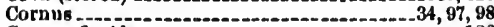

Cornus florida

Coruus pubescens ......................... 129

Corylus ..........81, 82, 99, 115, 117, 124, 125, 128, 134

Corylue americana.......................... 121

Cotton -......- 93

Crategur...............27, 38, 97, 106, 127.131, 135

Crategue tomentosa
Crotaiaria $\ldots \ldots \ldots \ldots$

$\begin{array}{llr}\text { Crotaiaria } & \text { Croton capitatum } \\ \text { Con }\end{array}$

Crueifere

Cucurbituceæ..........................50, 111, 112

Cupressus -..... 66

Cupressue thyoides 49

Cynoglossum officinale ..................... 56

Datura

Dictamnus -

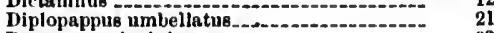

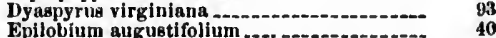

Epilobium augustifolium .................... $\quad 40$

$\begin{array}{ll}\text { Fricaces } & 10\end{array}$

Erodinm

Erodium cicntarium

Erodium cicntarium

Kupatorium

Eupatorium purpu reum ...................136, 137

Euphorbiaceæ (seed-pods) _..._......._._.... 120

Fagus _..._.

Fagus sylvatica ........ 117

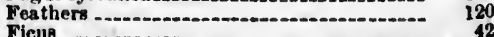

Fieus -

Fraxinus

Fraxinus
Fraxinut (stems and trunke) $\ldots \ldots . . . . . . . . .60,51$

(145) 


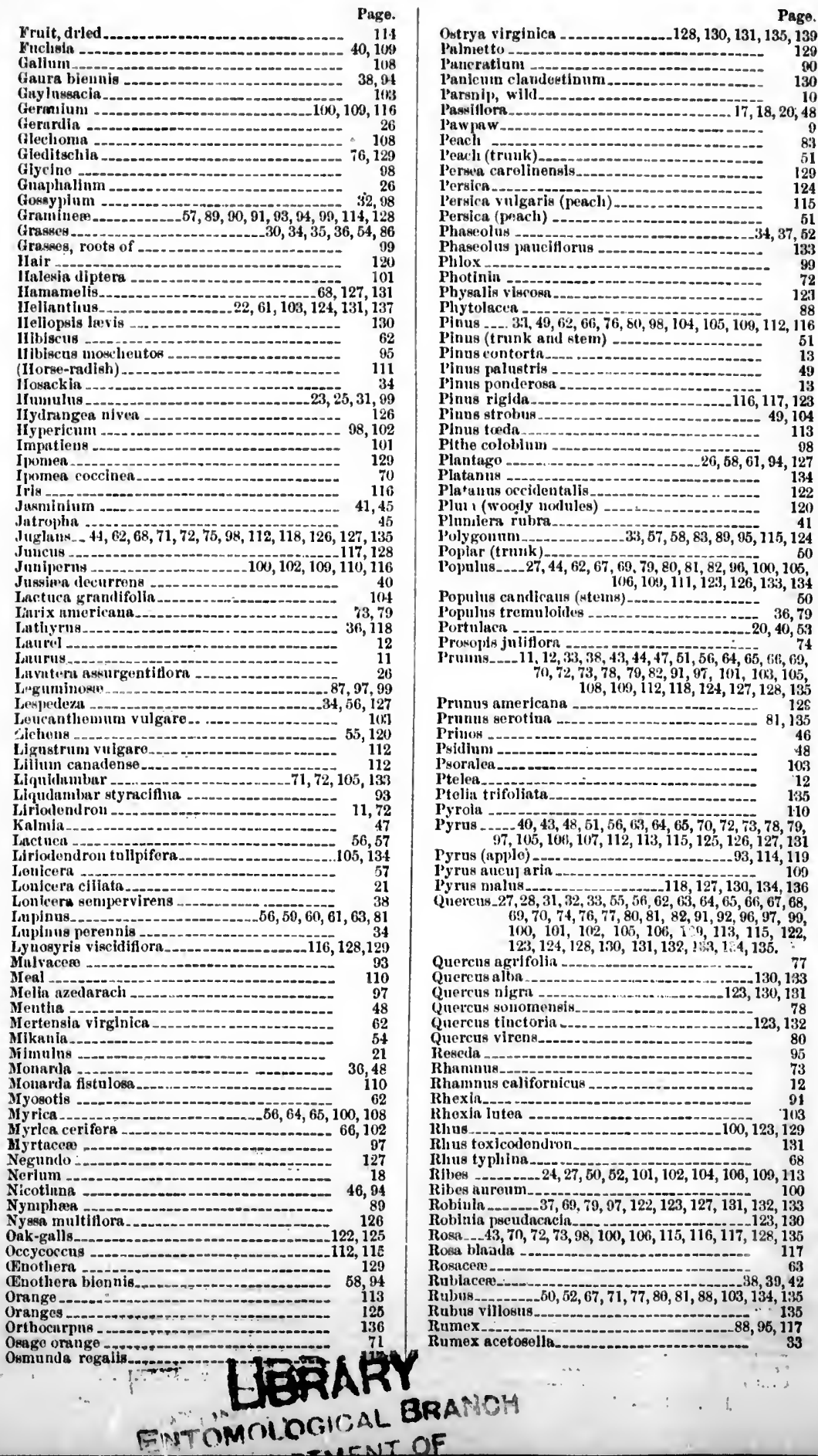


Pago.

Sailx sp, $12,24,25,27,31,43,44,50,55,61,62,64,66,67$ $68,69,70,74,79,80,82,91,92,96,98,1(02,105$, $108,109,111,114,117,118,126,148,129,132$ $133,134$.

Bailix galla

Eaman

Rambucus

Sambucue (stoms)

Sanicula

Salvia

Surruconia

Sasenafms

Saw-palmet to

Suxifraga

Bcroplunlaria.

Serophulariacea

Sentollaria

13,2

Sedum spatlunlifolinm

Senecio_._........

Sosamum

Slderonytum tenax

Silphimn Integrifolinm

Smitinx

Smilax giabra

Snilax rotundifolin....

Boianım -

Solanum carolinense

Solitago______ $59,86,92,104,122,124,1: 30,137$

Solidago (gailis) -.. 118

Spiren

Spirea salicifolia

Stachys palustris_..... 117

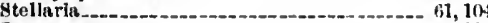

Sugar-cano

Symphoricarpus -

Symphoricarpus racemosug_..... 38

Symplioricarpus viligaris.

svrlnga

$47,48,67,83$

Syringa (trunk and stem)
$47,48,67,83$
Pago.

Tubernamontana laurifolia _.............. Taraxacum_........

Thalictrun coruutum................. 9 :

Tecoma radicans

Thistle _..._-

Thuja occidentails $\rightarrow \ldots$

Tllh _...

Tilla americana _.....-

Tragapod

Tritolium

Trillum stylosim

Triosteum perfoilitum

Turritis glabra

Typha latifolla -

Uimus _....... $23,64,65,69,72,81,99,106,107,109$

Ulnus americana _... 15, 131

Unubellifere _.............

Urena lobuta

- 92

Yaccinium -

Vaccinium corymbosum.

Vorbena............. 92,116

Vernonla

Yernonin neveborncensis

Viburnum _.......

Viburnum acerifollum.

Vicin...

Viola_...-

Vitis $\_39,41,43,53,54,55,91,103,109,112,117,125$, 136

Vitis corilifolia -. 134

Wlieat 12

Whliow (trunks)

Wistaria

Woolen fabrics _.__________________._._. 120, 121

Wyethis angustifolia

Yucen -.._. 37, 121

Zamia integrifolia

Zea (atems) _._. 
